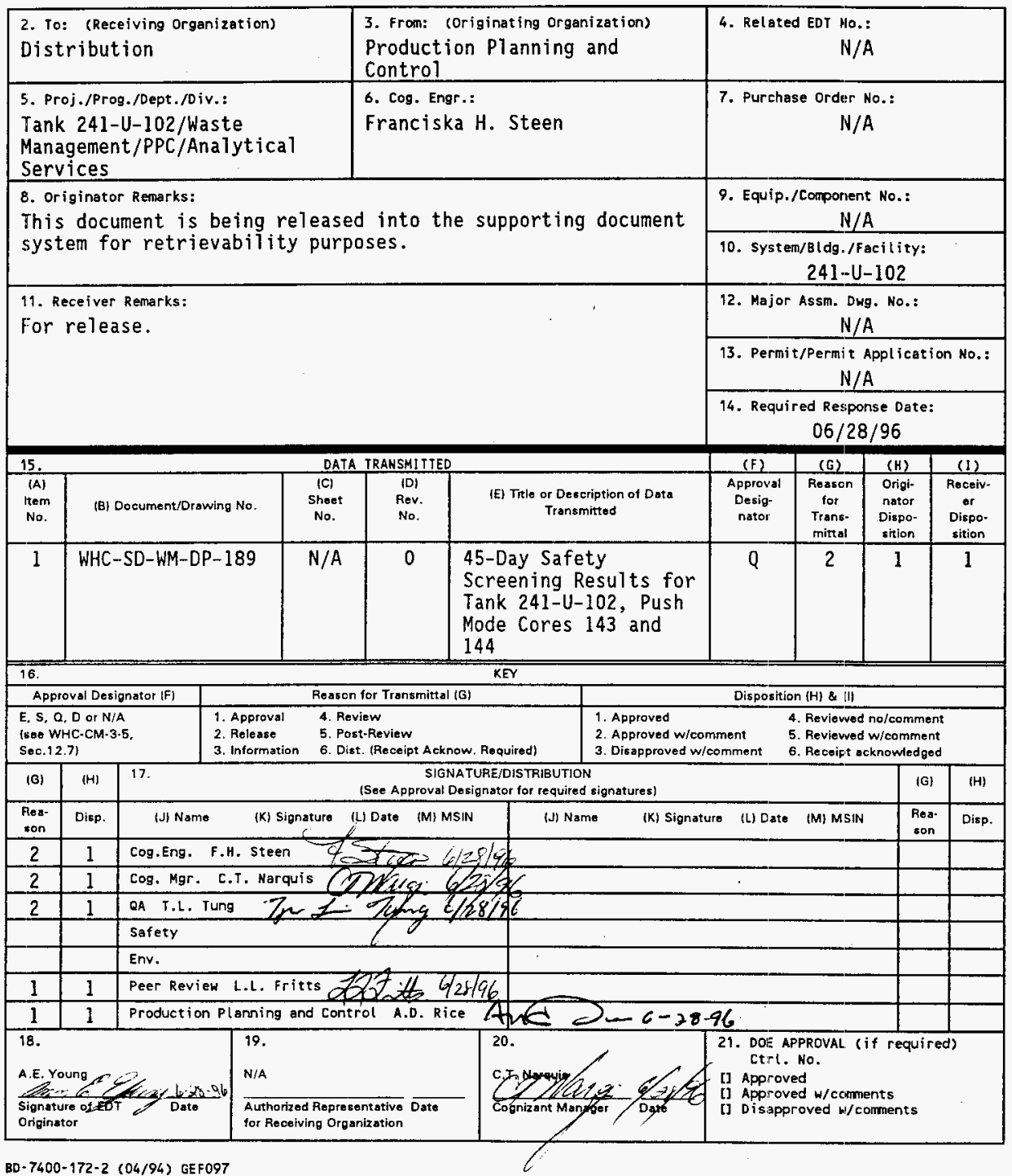




\section{"45-DAY SAFETY SCREENING RESULTS FOR TANK 241-U-102, PUSH MODE CORES 143 AND 144."}

Franciska H. Steen

Westinghouse Hanford Company, Richland, WA 99352

U.S. Department of Energy Contract DE-AC06-87RL10930

$\begin{array}{lll}\text { EDT/ECN: } & \text { EDT-614797 } & \text { UC: } 2070 \\ \text { Org Code: } & 75725 & \text { Charge Code: MD378 } \\ \text { B\&R Code: } & \text { EW } 3120074 & \text { Total Pages: 271 }\end{array}$

Key Words: 45-Day Safety Screening Results for Tank 241-U-102, Push Mode Cores 143 and 144, Tank 241-U-102 Push Mode

Abstract: N/A

TRADEMARK DISCLAIMER. Reference herein to any specific commercial product, process, or service by trade name, trademark, manufacturer, or otherwise, does not necessarily constitute or imply its endorsement, recommendation, or favoring by the United States Government or any agency thereof or its contractors or subcontractors.

Printed in the United States of America. To obtain copies of this document, contact: WHC/BCS

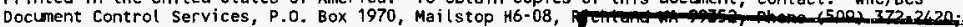
Fax (509) 376-4989.
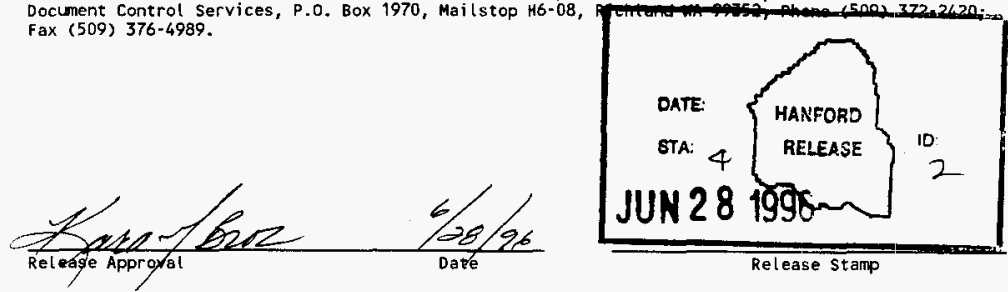

Release stamp

\section{Approved for Public Release}


WHC-SD-WM-DP-189, REV. 0

ANALYTICAL SERVICES

\title{
45-DAY SAFETY SCREENING RESULTS FOR \\ TANK 241-U-102, PUSH MODE CORES 143 AND 144
}

\author{
Project Coordinator: FRANCISKA H. STEEN \\ Prepared for the U.S. Department of Energy \\ office of Environmental Restoration \\ and Waste Management \\ by \\ Westinghouse Hanford Company \\ Box 1970 \\ Richland, Washington
}


WHC-SD-WM-DP-189, REV. 0

THIS PAGE. WAS INTENTIONALLY LEFT BLANK 
Narrative ........................... 1

Sample Data Summary . . . . . . . . . . . . . . . . . . 11

U-102 Push Mode Core Sample Breakdowns (Attachment 1) . . . . . . 28

Chain of Custody Forms . . . . . . . . . . . . . . . . . . 47

Inorganic Anatyses . . . . . . . . . . . . . . . . . . 67

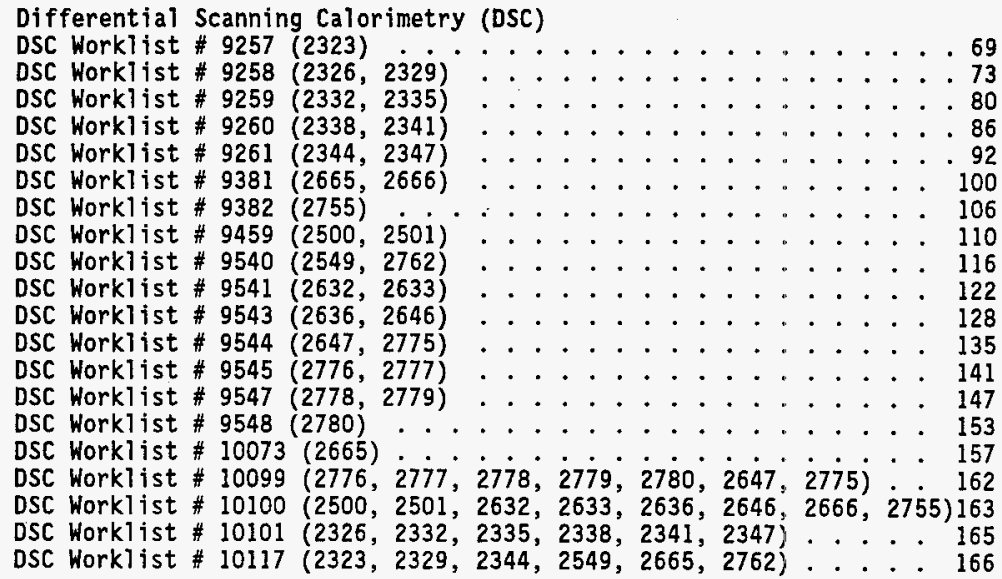

Thermogravimetric Analysis (TGA)

TGA Workl ist \#9248 (2323) . . . . . . . . . . . . . . . . 168

TGA Workl ist \# $9249(2329)$. . . . . . . . . . . . . . . . . 172

TGA Work list \# $9250(2332,2335)$. . . . . . . . . . . . . . . 176

TGA Workl ist \# $9251(2338,2341)$. . . . . . . . . . . . . . 182

TGA Workl ist \#9252 $(2344,2347)$. . . . . . . . . . . . . . . 188

TGA Workl ist \# $9383(2665,2666)$................ . . . 195

TGA Workl ist \#9384 (2755) ..................... . . . 201

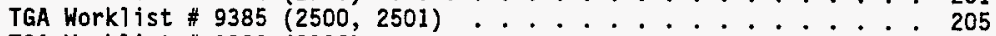

TGA Worklist \#9389 (2326) .................... 211

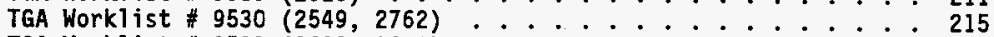

TGA Workl ist \#9531 $(2632,2633)$. . . . . . . . . . . . . . 221

TGA Workl ist \# $9532(2636,2646)$. . . . . . . . . . . . . . 227

TGA Worklist \#9533 $(2647,2775)$. . . . . . . . . . . . . . 234

TGA Worklist \#9534 $(2776,2777)$. . . . . . . . . . . . . . . . . 240

TGA Worklist \# $9535(2778,2779)$. . . . . . . . . . . . . . . 246

TGA Worklist \# $9536(2780)$. . . . . . . . . . . . . . . . 252

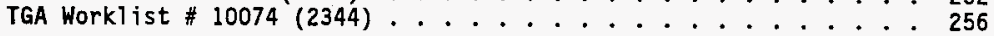




\section{WHC-SD-WM-DP-189, REY. 0}

This document consists of pages 1 through 259 and pages $i i, 2,12,29,48$, and 68 were intentionally left blank.

\section{TRADEMARKS:}

Perkin-Elmer is a Registered Trademark of Research and Manufacturing Company, Inc. Mettler is a Registered Trademark of Mettler Electronics 
WHC-SD-WM-DP-189, REV. 0

\section{NARRATIVE}


WHC-SD-WM-DP-189, REV. 0

THIS PAGE WAS INTENTIONALLY LEFT BLANK 
WHC-SD-WM-DP-189, REV.0

222-S ANALYTICAL SERVICES

\section{5-DAY SAFETY SCREENING RESULTS FOR TANK 241-U-102 CORES 143 AND 144}

This document is the 45-day report deliverable for tank 241-U-102 push mode core segments collected between April 16, 1996 and May 6, 1996 and received by the 222-S Laboratory between April 17, 1996 and May 8, 1996. The segments were subsampled and analyzed in accordance with the Tank 24/-U-102 Push Mode Core Sampling and Analysis Plan (TSAP) (Hu, 1996) and the Safety Screening Data Quality Objective (DQO) (Dukelow, et al., 1995). The analytical results are included in Table 1.

Attachment 1 is a cross reference to relate the tank farm identification numbers to the 222-S Laboratory LabCore sample numbers. The subsamples generated in the laboratory for analysis are identified in these diagrams with their sources shown. The diagram identifying the hydrostatic head fluid (HHF) blank is also included. Primary safety screening results and the raw data from Differential Scanning Calorimetry (DSC) and thermogravimetric analysis (TGA) analyses are included in this report.

Two of the samples submitted for DSC analysis exceeded notification limits as stated in the Safety Screening DQO (Dukelow, et al., 1995). Cyanide analysis was requested on these samples and a Reactive System Screening Tool analysis was requested for the sample exhibiting the highest exotherm in accordance with the TSAP (Hu, 1996). The results for these analyses will be reported in a revision to this document.

\section{Appearance and Sample Handling}

\section{Core 143}

Nine push mode core segments were removed from tank 241-U-102 riser 19 between April 16, 1996 and May 6, 1996. It should be noted that Segment 6A was sampled on May 6, 1996 following the collection of Core 144 segments; the sampler was empty. Segments were received by the 222-S Laboratory between April 17, 1996 and May 8, 1996. Three casks were received for segment 5: 5, 5A and 5B. Table 2 summarizes the extrusion information.

\section{Core 144}

Seven push mode core segments were removed from tank 241-U-102 riser 9 between April 26, 1996 and April 30, 1996. The segments were received by the 222-S Laboratory between April 30, 1996 and May 8, 1996. The results for DSC, TGA and total alpha analyses on segment 6A will be reported in an addendum to this report. This segment did not provide enough material to perform a bulk density analysis. Table 3 summarizes the extrusion information. 


\section{WHC-SD-WM-DP-189, REV.0}

\section{Field Blank}

A field blank was provided to the 222-S laboratory with core 144 . It underwent the same analysis as the drainable liquid as instructed by the TSAP (Hu, 1996).

\section{Hydrostatic Head Fluid}

Lithium bromide solution was provided to the 222-S laboratory with core 144 . It underwent Inductively Coupled Plasma Spectroscopy (ICP) and Ion Chromatography (IC) analysies as instructed by the TSAP (Hu, 1996). The results for these analyses will be reported in a revision to this document.

\section{Liner Liquid}

109.4 grams of liner liquid was recovered from Core 144 Segment 5. The TSAP does not address analyses for liner liquid. As per agreement with the TWRS representative, no analyses were performed and the sample was archived for possible future analyses. 


\section{WHC-SD-WM-DP-189, REV.0}

Table 2. Sample Receipt and Extrusion Information for 241-U-102, Core 143.

\begin{tabular}{|c|c|c|c|c|c|c|c|c|}
\hline W & X & 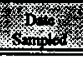 & 16on & 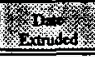 & Sying & 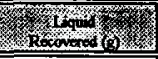 & 桨 & 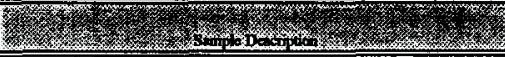 \\
\hline $96-182$ & $\mathbf{I}$ & $4 / 16 / 96$ & $4 / 23 / 96$ & $424 / 96$ & 6.0 & 105.7-Drainable & $\begin{array}{l}\text { 171.5-upper half } \\
\text { 49.0--10wer half }\end{array}$ & 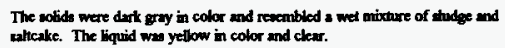 \\
\hline 96-183 & 2 & $4 / 16 / 96$ & $417 / 96$ & $4 / 24 / 96$ & 19.0 & 0.0 & $\begin{array}{l}\text { 228.9-4pper half } \\
\text { 172.2-1ower half }\end{array}$ & $\begin{array}{l}\text { The polids were medium gray in color and recembled a wet minture of studge } \\
\text { and salicake. }\end{array}$ \\
\hline 96-184 & 3 & $416 / 96$ & $4 / 17 / 96$ & $4 / 24 / 96$ & 19.0 & 2.0 & $\begin{array}{l}\text { 202.3-upper heif } \\
\text { 198.6-lower half }\end{array}$ & $\begin{array}{l}\text { The wolida were modium gry in color and resembled a wet mixture of aludge } \\
\text { and salicake. }\end{array}$ \\
\hline 96-189 & 4 & $4 / 16 \% 6$ & $4 / 23 / 96$ & $4 / 24 / 96$ & 19.0 & 0.0 & $\begin{array}{l}\text { 210.2-upper half } \\
\text { 206.2-10wer half }\end{array}$ & 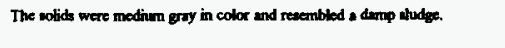 \\
\hline $96-186$ & 5 & $4 / 16 / 96$ & $4 / 23 / 96$ & $4 / 29 / 96$ & 5.0 & 109.4-Liner & 80.6-whok segment & 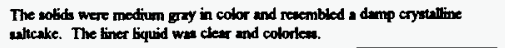 \\
\hline $9-186 \mathrm{~A}$ & $3 A$ & $4 / 6 / 96$ & $4 / 17 / 96$ & $4 / 29 / 96$ & 8.0 & 0.0 & 186.9-upper half & 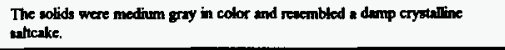 \\
\hline $96-186 \mathrm{~B}$ & 5B & $4 / 2296$ & $4 / 30196$ & $5 / 07 / 96$ & 12.0 & 0.0 & $\begin{array}{l}\text { 108.4-upper half } \\
\text { 170.4-lower half }\end{array}$ & $\begin{array}{l}\text { The robids were light to medium gray in coler and resembled a wet cryatrlibine } \\
\text { salteake. }\end{array}$ \\
\hline $96-187 \mathrm{~A}$ & $6 \mathrm{~A}$ & $5 / 06 / 96$ & $5 / 08 / 96$ & $5 / 13 / \%$ & 0.0 & 0.0 & 0.0 & Samplet emply. \\
\hline
\end{tabular}

-Approximate Inches Exaruded 


\section{WHC-SD-WM-DP-189, REV.0}

Table 3. Sample Receipt and Extrusion Information for 241-U-102, Core 144.

\begin{tabular}{|c|c|c|c|c|c|c|c|c|}
\hline 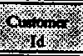 & xising & 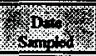 & 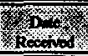 & 1. & 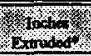 & 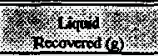 & Xkon & 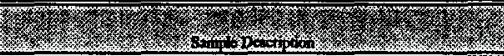 \\
\hline $\begin{array}{l}\text { Field } \\
\text { Blenk }\end{array}$ & FB & $4 / 29 / 96$ & $\operatorname{sins/96}$ & $5 / 13 / 96$ & 0.0 & 212.6-drainstbe & 0.0 & The trainable bquid wa colorien and cken. \\
\hline 96-189 & 1 & $426 / 96$ & $4 / 30 / 96$ & $5 / 06 / 96$ & 3.0 & 303.3-drainable & 63.5 & $\begin{array}{l}\text { The drainable liquid was grayish brown in color and opaque. The solids were } \\
\text { grayith brown in color and rescmbled a wer cryastline salteake. }\end{array}$ \\
\hline 96.190 & $\mathbf{2}$ & $426 \%$ & 430196 & $5 / 06 / \%$ & 16.0 & 0.0 & $\begin{array}{l}\text { 169.7-upper half } \\
\text { 205.1 tower half }\end{array}$ & 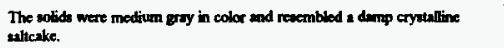 \\
\hline 9-191 & 3 & $4 / 29 / 96$ & $4 / 3096$ & $5 / 06 / 96$ & 14.0 & 0.0 & $\begin{array}{l}\text { 167.3-upper half } \\
\text { 149.5-lower half }\end{array}$ & $\begin{array}{l}\text { The aotide mere mediun gray in color and resemblod a dump crystalline } \\
\text { salticale. }\end{array}$ \\
\hline 9-192 & 4 & $4 / 29 / 96$ & $5 / 08 / 96$ & $5 / 13 / \%$ & 19.0 & 0.0 & $\begin{array}{l}\text { 234.6-upper half } \\
\text { 224.4-lower half }\end{array}$ & The solids were medium gray in color and resembled a wet wht. \\
\hline $96-193$ & 5 & 4/29/96 & $5 / 08 / 96$ & $5 / 13 / 96$ & 18.0 & 0.0 & $\begin{array}{l}\text { 182.8-upper half } \\
\text { 237.3-lower hatf }\end{array}$ & The notids were modium gray in color mod resembled a moint with. \\
\hline 96-194 & 6 & $4 / 30 / 96$ & 5/0896 & $5 / 13 / 96$ & 13.0 & 0.0 & $\begin{array}{l}\text { 134.6-upper half } \\
\text { 161.2-lower half }\end{array}$ & $\begin{array}{l}\text { The sotids were ligth oray wo medium gray in color and resembled a moin } \\
\text { ealt. }\end{array}$ \\
\hline
\end{tabular}




\section{Extruded Results Summary}

The data summary table (Table 1) included in this report compiles the safety screening analytical results and applicable action limits associated with each subsample submitted.

\section{Differential Scanning Calorimetry (DSC)}

Two of the samples submitted for DSC analysis exceeded notification limits as stated in the Safety Screening DQO (Dukelow, et al., 1995). Cyanide analysis was requested on these samples and a Reactive System Screening Tool analysis was requested for the sample exhibiting the highest exotherm in accordance with the TSAP (Hu, 1996). The results for these analyses will be reported in a revision to this document.

The DSC analyses were performed in duplicate on direct subsamples. The exothermic energy based on dry weight of subsample was calculated for all subsamples. The average of the TGA results for each subsample was used in the dry weight correction for that subsample. The standard recovery for this analysis was within the required limits.

The results for six of the twenty-four subsamples were the sum of two or more exotherms. More information may be obtained by examining the raw data. The field blank result was $0.00 \mathrm{Joules} / \mathrm{g}$.

Relative percent differences (RPD) greater than $20 \%$ were reported for twelve of the twenty-four subsamples. The high RPDs can be attributed to the small exotherms and the heterogenous nature of the samples. Selected samples had triplicate or rerun analyses performed because of the high RPDs and differences in the appearance between the thermograms of the sample and duplicate. The results of the triplicate wet weight DSCs are presented in Table 4. The results of the triplicate dry weight corrected DSCs are presented in Table 5. The results of the reruns can be found in the analytical results summary (Table 1).

Review of the data by the TWRS representative resulted in a request for DSC renuns on the following samples: S96T002646 and S96T002778. These results will be reported in a revision to this document. 
WHC-SD-WM-DP-189, REV.0

Table 4. Triplicate Results for Wet Weight DSC Analysis

\begin{tabular}{|c|c|c|c|c|c|}
\hline 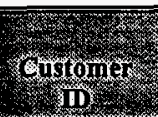 & 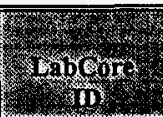 & 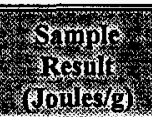 & $\begin{array}{l}\text { ourpicits } \\
\text { pesut } \\
\text { (coules) }\end{array}$ & 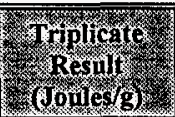 & 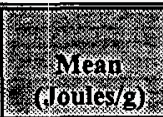 \\
\hline & $\mathrm{S} 96 \mathrm{~T} 002344$ & 0.0 & 11.7 & 16.3 & 9.3 \\
\hline
\end{tabular}

Table 5. Triplicate Results for Dry Weight DSC Analysis

\begin{tabular}{|c|c|c|c|c|c|}
\hline Sustomer & hing cor & 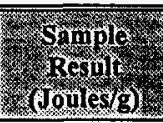 & 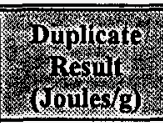 & $\begin{array}{l}\text { Triplicart } \\
\text { Resuit } \\
\text { (Joules) }\end{array}$ & 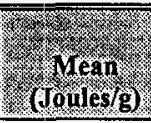 \\
\hline $96-185$ & S96T002344 & 0 & 15.45 & 16.3 & 10.6 \\
\hline
\end{tabular}

\section{Thermogravimetric Analysis (TGA)}

The TGA analyses were performed in duplicate on direct subsamples. Typically results were determined by summing the weight loss steps which occurred below $200^{\circ} \mathrm{C}$; weight loss steps above this were not used to determine the result with the following exceptions: the sample and duplicate results for S96T002776 and S96T002777 were the result of weight loss steps up to $420^{\circ} \mathrm{C}$. The chemist noted that the data could not be retrieved from the data disk to reintegrate the results. The chemist also noted that had the sample results been integrated to $200^{\circ} \mathrm{C}$, the results would have differed from the existing results by less than $2 \%$. More information may be obtained by examining the raw data.

The field blank resulted in a mean of $99.45 \%$ moisture. Relative percent differences (RPD) greater than $20 \%$ were reported for five of the twenty-four subsamples. The high RPDs suggest a wide variance in sample matrix. Selected samples had reruns performed because of the high RPDs and differences in the appearance between the thermograms of the sample and duplicate. The results of the reruns can be found in the analytical results summary (Table 1). The standard recovery for this analysis was within the required limits. 
Review of the data by the TWRS representative resulted in a request for TGA reruns on the following samples: S96T002344, S96T002646, S96T002647 and S96T002636. These results will be reported in a revision to this document.

\section{Density}

Bulk density was performed on all of the twenty-four solid subsamples as required by the TSAP (Hu, 1996). The results of the bulk density test ranged from $1.55 \mathrm{~g} / \mathrm{mL}$ to $1.88 \mathrm{~g} / \mathrm{mL}$. The higher bulk density of $1.88 \mathrm{~g} / \mathrm{mL}$ was used to calculate the solid total alpha activity action limit for the tank.

Total alpha results for liquids do not require correction for density. The specific gravity results for the liquid samples will be reported in a revision to this document.

\section{Total Alpha (AT)}

The total alpha (AT) analyses were performed in duplicate on direct subsamples for the liquids. Solid subsamples were prepared for analysis by performing a fusion digest in duplicate. The fusion digest is indicated with an " $F$ " in the aliquot class (A\#) column in Table 1.

All liquid AT results were below the total alpha activity action limit of $61.5 \mu \mathrm{Ci} / \mathrm{mL}$. All solid AT results were below the total alpha activity limit of $32.7 \mu \mathrm{Ci} / \mathrm{g}$ (based on a bulk density of 1.88 $\mathrm{g} / \mathrm{mL}$ ). The field blank result was less than $4.62 \mathrm{e}-6 \mu \mathrm{Ci} / \mathrm{mL}$. A high spike recovery was reported for sample S96T002549 and a low spike recovery was reported for sample S96T002662. This is attributable to low alpha activity and signifies no compromise in data quality. The standard recovery and $R P D$ s for this analysis were within the required limits. 


\section{WHC-SD-WM-DP-189, REV.0}

\section{Procedures}

Table 6 lists the analytical procedures used for performing the sample analyses. Abbreviations for analyses are defined in the table notes.

Table 6. Analytical Procedures

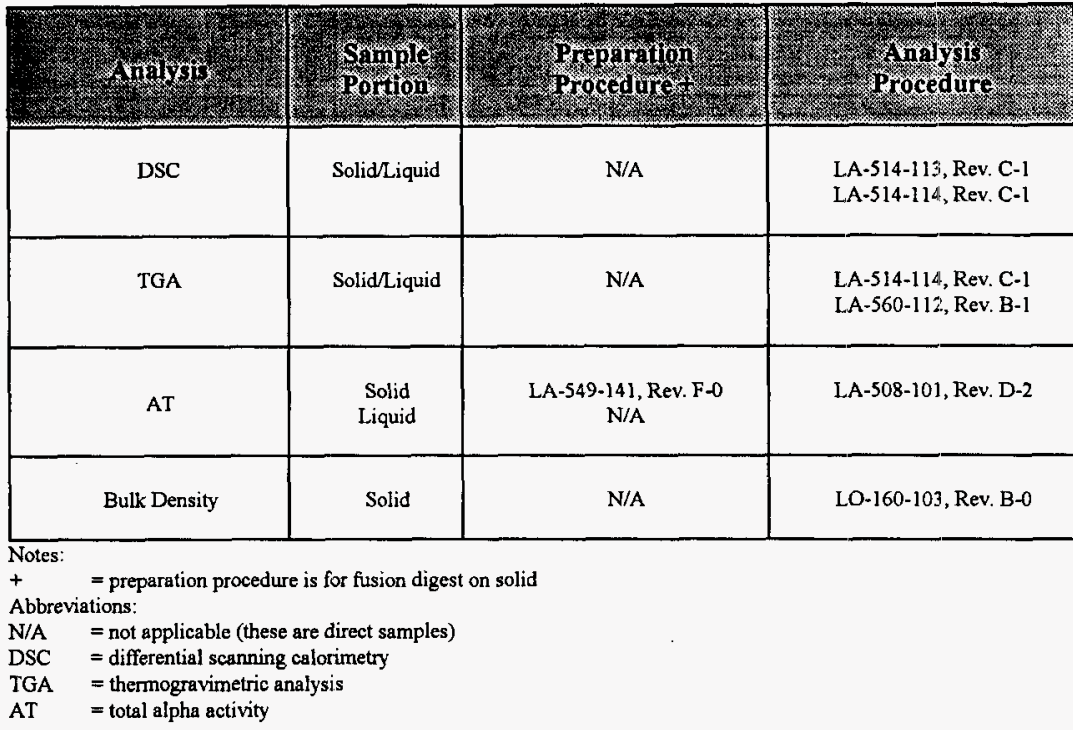

\section{References}

Hu, T. A., 1996, Tank 241-U-102 Push Mode Core Sampling and Analysis Plan, WHC-SD-WM-TSAP-082, Rev. 0-A, Westinghouse Hanford Company, Richland, WA 99352.

Dukelow, G. T., J. W. Hunt, H. Babad, and J. E. Meacham, 1995, Tank Safety Screening Data Quality Objective, WHC-SD-WM-SP-004, Rev. 2, Westinghouse Hanford Company, Richland, WA 99352 
WHC-SD-WM-DP-189, REV. 0

SAMPLE DATA SUMMARY 
WHC-SD-WM-DP-189, REV. 0

THIS PAGE WAS INTENTIONALLY LEFT BLANK 
CORE NUMBER: 143

SEGMENT H: $\uparrow$

SEGMENT PORTION: U Upper Half of Segnent

\begin{tabular}{|c|c|c|c|c|c|c|c|c|c|c|c|c|c|c|}
\hline \multirow[b]{2}{*}{ Samplet $R$} & \multirow[b]{2}{*}{ A\# } & \multirow[b]{2}{*}{ Analyte } & \multirow[b]{2}{*}{ Unit } & \multicolumn{2}{|c|}{ Action Limits } & \multirow[b]{2}{*}{ Standard \% } & \multirow[b]{2}{*}{$B$ lank } & \multirow[b]{2}{*}{ Result } & \multirow[b]{2}{*}{ Duplicate } & \multirow[b]{2}{*}{ Average } & \multirow[b]{2}{*}{ RPD \% } & \multirow[b]{2}{*}{ Spk Rec \% } & \multirow[b]{2}{*}{ Det Limit } & \multirow[b]{2}{*}{ Count Err\% } \\
\hline & & & & Lower & Upper & & & & & & & & & \\
\hline 5961002325 & & Bulk Density of Sample & $9 / \pi \mathrm{mL}$ & None & None & $n / a$ & $n / a$ & 1.610 & $n / a$ & $n / a$ & $n / a$ & $\mathrm{n} / \mathrm{a}$ & $5.00 \mathrm{e}-01$ & $n / a$ \\
\hline$\frac{5961002326}{5967002326}$ & & DSC Exotherm Dry Calcutated & Joutes/g Dry & $-1.0 e+03$ & 84000 & $\mathrm{n} / \mathrm{a}$ & $n / a$ & $1.18 \mathrm{e}+02$ & 112.7 & 115.4 & 4.68 & $n / a$ & $n / a$ & $n / a$ \\
\hline$\frac{5961002526}{5967002326}$ & & $\begin{array}{l}\text { DSC Exotherm on Perkin Etmer } \\
\text { \% water by IGA using Mettler }\end{array}$ & Joules/g & $-1.0 \mathrm{e}+03$ & \%80.8. & 97.36 & $n / a$ & 69.80 & 66.60 & 68.20 & 4.69 & $n / a$ & $n / a$ & n/ \\
\hline & & & $\%$ & None & None & 98.89 & $n / a$ & 41.50 & 40,30 & 40.90 & 2.93 & $n / a$ & $n / a$ & $\mathrm{n} /$ \\
\hline
\end{tabular}

L Lower Half of Segment: L Lower Half of Segment

\begin{tabular}{|c|c|c|c|c|c|c|c|c|c|c|c|c|c|c|}
\hline Sample\# & A\# & Analyte & Unit & $\begin{array}{c}\text { Action } \\
\text { Lower }\end{array}$ & $\frac{\text { imits }}{\text { Upper }}$ & Standard \% & 8lank & Result & Dupl icate & Average & RPD $*$ & Spk Rec \% & Det Limit & Count Err\% \\
\hline 5961002328 & & Bulk Density of Sample & $\mathrm{g} / \mathrm{ml}$ & None & None & $n / a$ & $n / a$ & 1.810 & n/a & $n / a$ & $n / a$ & $\mathrm{n} / \mathrm{a}$ & $5.00 \mathrm{e}-01$ & $n / a$ \\
\hline \begin{tabular}{|l}
5961002329 \\
5961002329
\end{tabular} & & & $\begin{array}{l}\text { Joules/g_Dry } \\
\text { Joules } / g\end{array}$ & $\frac{-1.0 \mathrm{e}+03}{-1.0 \mathrm{e}+03}$ & 480.0 & $n / a$ & $n / a$ & $1,10 \mathrm{e}+02$ & 106.8 & 108.2 & 2.59 & $n / a$ & $n / a$ & $n / a$ \\
\hline 5961002329 & & $\begin{array}{l}\text { USC Exotherm on Perkin Elmer } \\
\text { \& Water by TGA on Perkin Elmer }\end{array}$ & $\frac{\text { Joules} / 9}{\%}$ & \begin{tabular}{|l|}
$-1.0 e+03$ \\
\end{tabular} & 48040 & 97.36 & $\mathrm{n} / \mathrm{a}$ & .53 .10 & 51.70 & 52.40 & 2.67 & $n / a$ & $n / a$ & $n / a$ \\
\hline 5961002443 & $F$ & Alpha of Digested solid & $u \bar{i} / \mathrm{g}$ & $\frac{-1.0 \mathrm{e}+03}{\mid-03}$ & None & $\frac{99.31}{101.6}$ & $\begin{array}{r}n / a \\
48.69 e^{-0}-03\end{array}$ & $\begin{array}{r}51.79 \\
5.56 e-02\end{array}$ & $\begin{array}{r}51.35 \\
5.91 e^{-02}\end{array}$ & $\frac{51.57}{5.73 e-02}$ & $\frac{0.85}{6.10}$ & $\begin{array}{r}n / a \\
90.25\end{array}$ & $\frac{n / a}{1.10 e^{-02}}$ & $\frac{n / a}{2.70 E+01}$ \\
\hline
\end{tabular}

Drainable Liquid: Drainable Liquid

\begin{tabular}{|c|c|c|c|c|c|c|c|c|c|c|c|c|c|c|}
\hline Sample\# R & A\# & Analyte & Unit & Action & $\frac{\text { Limfts }}{\text { Upper }}$ & Standard & Blank & Result & Dupl icate & Average & RPD \% & Spk Rec $\%$ & Det Limit & Count Err\% \\
\hline 5961002323 & & DSC Exotherm using Mettler & Joules/g & $-1.0 \mathrm{e}+03$ & 4000 & $111 \%$ & $n / a$ & $1.22 \mathrm{e}+02$ & 124.1 & 123.0 & 1.79 & $n / a$ & $n / a$ & $n / a$ \\
\hline$\frac{5961002323}{5061002373}$ & & & Joules/g Dry & $-1.0 \mathrm{e}+03$ & 848040 & $n / a$ & $n / a$ & $2.47 \mathrm{e}+02$ & 251.3 & 249.1 & 1.81 & $\mathrm{n} / \mathrm{a}$ & $n / a$ & $n / a$ \\
\hline$\frac{5961002323}{5961002323}$ & & \begin{tabular}{|l} 
\% Water by TGA using Mettler \\
Atpha in Liquid Samples
\end{tabular} & $\%$ & $\begin{array}{r}\text { None } \\
1.00+03\end{array}$ & None & $\begin{array}{r}98 \% 07 \\
111.7\end{array}$ & $\frac{n / a}{<1.24 e^{-02}}$ & $\begin{array}{r}50.93 \\
2.72 e .02\end{array}$ & 50.28 & 50.61 & 1.28 & $\mathrm{n} / \mathrm{a}$ & $n / a$ & $n / a$ \\
\hline
\end{tabular}

\section{W...... $\Rightarrow$ Limit violated}

$\Rightarrow$ Selected Limit

is 
CORE MUMBER: 143

$$
\text { U-102 }
$$

SEGMENT \#: 2

\begin{tabular}{|c|c|c|c|c|c|c|c|c|c|c|c|c|c|c|}
\hline \multirow[b]{2}{*}{ Sample\# } & \multirow[b]{2}{*}{$R$ A\# } & \multirow[b]{2}{*}{ Analyte } & \multirow[b]{2}{*}{ Unit } & \multicolumn{2}{|c|}{ Action Limits } & \multirow[b]{2}{*}{ standard \% } & \multirow[b]{2}{*}{ Bl ank } & \multirow[b]{2}{*}{ Result } & \multirow[b]{2}{*}{ Duplicate } & \multirow[b]{2}{*}{ Average } & \multirow[b]{2}{*}{ RPD $\%$} & \multirow[b]{2}{*}{ Spk Rec $\mathrm{z}$} & \multirow[b]{2}{*}{ Det Limit } & \multirow[b]{2}{*}{ Count Err\% } \\
\hline & & & & Lower & Upper & & & & & & & & & \\
\hline 5961002331 & & Bulk Dens ity of Sample & $\mathrm{g} / \mathrm{mL}$ & None & Hone & $n / a$ & $n / a$ & 1.690 & $\mathrm{n} / \mathrm{a}$ & $n / a$ & $n / a$ & n/a & $5.00 \mathrm{e}-01$ & $n / a$ \\
\hline 5961002332 & & DSC Exotherm using Mettler & Joules/g & $-1.0 \mathrm{e}+03$ & 6800 & 111.8 & $\mathrm{n} / \mathrm{a}$ & $2.36 e+02$ & 288.9 & 262.6 & 20.0 & n/a & $n / a$ & $n / a$ \\
\hline 5961002332 & & DSC Exotherm Dry Calculated & Joules/g Dry & $-1.0 e+03$ & 280. & $n / a$ & $n / a$ & $4.81 \mathrm{e}+02$ & 587.9 & 534.4 & 20.0 & $\mathrm{n} / \mathrm{a}$ & $\mathrm{n} / \mathrm{a}$ & $n / a$ \\
\hline$\$ 961002332$ & & \% Hater by TGA using Meteler & $\%$ & None & None & 97.94 & $n / a$ & 50,30 & 51.41 & 50.85 & 2.18 & $\mathrm{n} / \mathrm{a}$ & $\mathrm{n} / \mathrm{a}$ & $n / a$ \\
\hline
\end{tabular}

L Lower Half of Segment: L Lower Half of Segment

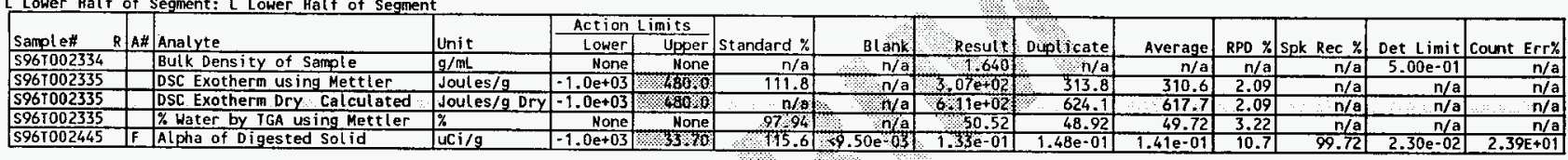

$\Rightarrow$ Limit violated

$\Rightarrow$ Selected Limit

$\underset{1}{4}$ 
CORE MUMBER: 143

SEGMENT \#: 3

SEGMENT PORTION: U Upper Half of Segment

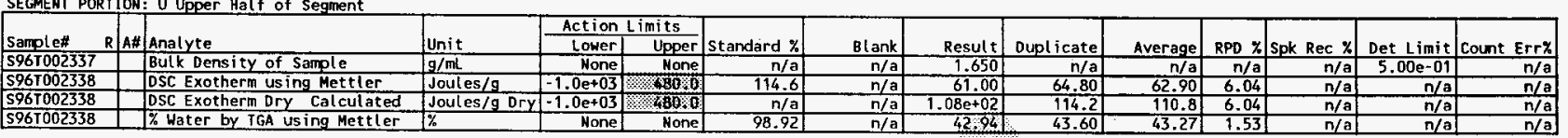

\begin{tabular}{|c|c|c|c|c|c|c|c|c|c|c|c|c|c|c|}
\hline & & & & Action & Limits & & & & & & & & & \\
\hline Sample\# & R A A\# & Analyte & Unit & Lawer & Upper & standard \% & Blank & Result: & Duplicate & Average & RPD $x$ & Spk Rec $\mathbf{x}$ & Det Limit & Count Err\% \\
\hline $596 \mathrm{~T} 002340$ & & Butk Density of Sample & $g / m L$ & \begin{tabular}{|l|} 
None \\
\end{tabular} & None & $n / a$ & $n / 6$ & 1640 & $\quad \mathrm{n} / \mathrm{a}$ & $n / a$ & $n / a$ & $n / a$ & $5.00 \mathrm{e}-01$ & $n / a$ \\
\hline 5967002341 & & DSC Exotherm using Mettler & Joules $/ \mathrm{g}$ & $-1.0 \mathrm{e}+03$ & 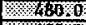 & 114.6 & $\mathrm{n} / \mathrm{a}$ & 65.70 & 86.50 & 76.10 & 27.3 & $n / a$ & $n / a$ & $n / a$ \\
\hline S967002341 & & DSC Exotherm Dry calculated & Joules/g Dry & $-1.0 \mathrm{e}+03$ & 240 & $n / 8$ & $\mathrm{n} / \mathrm{a}$ & 97.17 & 127.9 & 112.5 & 27.3 & $\mathrm{n} / \mathrm{a}$ & $\mathrm{n} / \mathrm{a}$ & $\mathrm{n} / \mathrm{a}$ \\
\hline S96T002341 & & $\%$ Hater by TGA using Mettler & $\%$ & None & None & 98,92 & o $\quad \mathrm{a}$ & 31.61 & 33.17 & 32.39 & 4.82 & $n / a$ & $\mathrm{n} / \mathrm{a}$ & $n / a$ \\
\hline 5967002447 & IF & Alpha of Digested Solid & UCi/g & $-1.0 \mathrm{e}+03$ & $\%$ & पत5.6 & $49.50 e^{-0}-03$ & $1.47 \mathrm{e}-01$ & $1.54 e-01$ & $1.50 \mathrm{e}-01$ & 4.65 & $n / a$ & $2.30 \mathrm{e}-02$ & $2.28 \mathrm{E}+01$ \\
\hline
\end{tabular}

1.......... $\Rightarrow$ Limit violated $\Rightarrow$ Selected Limit

6 
CORE NUMBER: 143

SEGMENT \#: 4

SEGMENT PORTION: U UpPer Half of Segment

\begin{tabular}{|c|c|c|c|c|c|c|c|c|c|c|c|c|c|c|}
\hline & & & & Action & imits & & & & & & & & & \\
\hline Sample\# & AH: & Analyte & Unit & Lower & Upper & standard \% & Blank & Result & Dupl icate & Average & RPD \% & Spk Rec \% & Det Limit & Count Err\% \\
\hline 5967002343 & & Bulk Density of Sample & $\mathrm{g} / \mathrm{mL}$ & None & None & $\mathrm{n} / \mathrm{a}$ & $n / a$ & 1.620 & $n / a$ & $n / a$ & $n / a$ & $n / a$ & $5.00 \mathrm{e}-01$ & $n / a$ \\
\hline 5961002344 & & DSC Exotherm Dry Calculated & Joules/gDry & $-1.0 \mathrm{e}+03$ & 6800 & $n / a$ & $n / a$ & $0.00 \mathrm{e}+00$ & 15.45 & 7.725 & 200 & n/a & $\mathrm{n} / \mathrm{a}$ & $n / a$ \\
\hline $596 \mathrm{~T} 002344$ & & DSC Exotherm on Perkin Elmer & Joules/g & $-1.0 \mathrm{e}+03$ & 4800 & 98.84 & $n / a$ & $0.00 \mathrm{e}+00$ & 11.70 & 5.850 & 200 & $n / a$ & $n / a$ & $n / a$ \\
\hline S96T002344 & & $\%$ Water by IGA on Perkin Elmer & & None & None & 98.46 & $n / a$ & 2244 & 14.78 & 18.61 & 41.2 & $n / a$ & $\mathrm{n} / \mathrm{a}$ & $n / a$ \\
\hline 59670023441 & & \% Water by IGA on Perkin ELmer & $\%$ & Mone & None & 100.4 & $n / a$ & 28.10 & 31.81 & 29.95 & 12.4 & n/a & $n / a$ & $\mathrm{n} / \mathrm{a}$ \\
\hline
\end{tabular}

$L$ Lower Half of Segment: L Lower Half of Segment

\begin{tabular}{|c|c|c|c|c|c|c|c|c|c|c|c|c|c|c|}
\hline \multirow[b]{2}{*}{ Sample\# } & \multirow[b]{2}{*}{$R|A \#|$} & \multirow[b]{2}{*}{ Analyte } & \multirow[b]{2}{*}{ Unit } & \multicolumn{2}{|c|}{ Action Limits } & \multirow[b]{2}{*}{ Standard \% } & \multirow[b]{2}{*}{$\mathrm{Btank}$} & \multirow[b]{2}{*}{ Result: } & \multirow[b]{2}{*}{ Dupticate } & \multirow[b]{2}{*}{ Average } & \multirow[b]{2}{*}{ RPD $\%$} & \multirow[b]{2}{*}{ Spk Rec \% } & \multirow[b]{2}{*}{ Det Limit } & \multirow[b]{2}{*}{ Count Err\% } \\
\hline & & & & Lower & Upper & & & & & & & & & \\
\hline 5967002346 & & Bulk Density of Sample & $\mathrm{g} / \mathrm{mL}$ & None & None & $n / a$ & $n / 3$ & 1.610 & $\mathrm{n} / \mathrm{a}$ & $n / a$ & $n / a$ & n/a & $5.00 \mathrm{e}-01$ & $n / a$ \\
\hline 5967002347 & & DSC Exotherm Dry Calculated & Joules/g ory & $-1.0 \mathrm{e}+03$ & 48009 & $n / 8$ & $\mathrm{n} / \mathrm{a}$ & $0 \% 00 \mathrm{e}+00$ & 104.0 & 52.00 & 200 & $n / a$ & $n / a$ & $n / a$ \\
\hline 5967002347 & & DSC Exotherm on Perkin Elmer & joules/g & $-1.0 \mathrm{e}+03$ & $8480 \% 60$ & 98.84 & na & $0.000+00$ & 57.20 & 28.60 & 200 & $\mathrm{n} / \mathrm{a}$ & $\mathrm{n} / \mathrm{a}$ & $n / a$ \\
\hline $596 T 002347$ & & \% Water by IGA on Perkin El mer & $\%$ & None & None & 98.46 & nzo & 46.82 & 43.20 & 45.01 & 8.04 & $\mathrm{n} / \mathrm{a}$ & $n / a$ & $n / a$ \\
\hline $596 \mathrm{TO} 002449$ & $\mathbf{F}$ & Alpha of oigested Solid & UCi/g & $-1.0 \mathrm{e}+03$ & 33 & 109.4 & $55.25 e-03$ & $2.13 e-01$ & $2.36 \mathrm{e}-01$ & $2.24 \mathrm{e}-01$ & 10.2 & n/a & $.00 e^{-03}$ & $0 \mathrm{E}+01$ \\
\hline
\end{tabular}

$\Rightarrow$ Limit violated

$\Rightarrow$ Selected Limi

in 
CORE NUMBER: 143

SEGMENT \# : 5

SEGMENI PORTION: W Whole Segment

\begin{tabular}{|c|c|c|c|c|c|c|c|c|c|c|c|c|c|c|}
\hline \multirow{2}{*}{ Sample\# R } & \multirow{2}{*}{$\begin{array}{lll} & \\
\end{array}$} & \multirow[b]{2}{*}{ Analyte } & \multirow[b]{2}{*}{ Unit } & \multicolumn{2}{|c|}{ Action Limits } & \multirow[b]{2}{*}{ Standard \% } & \multirow[b]{2}{*}{ Blank } & \multirow[b]{2}{*}{ Result } & \multirow[b]{2}{*}{ Duplicate } & \multirow[b]{2}{*}{ Average } & \multirow[b]{2}{*}{ RPO \% } & \multirow[b]{2}{*}{ Spk Rec \% } & \multirow[b]{2}{*}{ Det Limit } & \multirow[b]{2}{*}{ Count Err\% } \\
\hline & & & & Lower & Upper & & & & & & & & & \\
\hline $596 \mathrm{~T} 002498$ & & Bulk Density of Sample & $\mathrm{g} / \mathrm{mL}$ & None & Hone & $n / a$ & $n / a$ & 1.880 & $n / a$ & $n / a$ & $n / a$ & $n / a$ & $5.00 \mathrm{e}-01$ & $n / a$ \\
\hline 5967002500 & & DSC Exotherm using Mettler & Joules/g & $-1.0 \mathrm{e}+03$ & 680:00 & 101.9 & $n / a$ & 16.60 & 15.50 & 16.05 & 6.85 & $n / a$ & $n / \mathrm{a}$ & $n / a$ \\
\hline 5961002500 & & DSC Exotherm Dry Calculated & Joules/g Dry & $-1.0 \mathrm{e}+03$ & m80 & $n / a$ & $n / a$ & 19.79 & 18.47 & 19.13 & 6.90 & $\mathrm{n} / \mathbf{a}$ & $n / a$ & $n / a$ \\
\hline 5961002500 & & \% water by TGA using Mettler & $\%$ & None & Hone & 98.89 & $n / a$ & 15.72 & 16.48 & 16.10 & 4.72 & $n / a$ & $n / a$ & $n / a$ \\
\hline 5961002518 & $F$ & Alpha of oigested Solid & $u C i / g$ & $-1.0 \mathrm{e}+03$ & B. & 109.4 & $<5.25 e^{-03}$ & $8.316-02$ & $6.54 \mathrm{e}-02$ & $7.42 \mathrm{e}-02$ & 23.8 & 93.31 & $7.00 \mathrm{e}-03$ & $1.90 \mathrm{E}+01$ \\
\hline
\end{tabular}

$\Rightarrow$ Limit violated

$\Rightarrow$ Selected Limit

ix 
28- jun-1996 11:28:24 A- $0002-3$

SEGMERT \#: 5 A

SEGMEMT PORTION: U Upper Half of Segment

\begin{tabular}{|c|c|c|c|c|c|c|c|c|c|c|c|c|c|c|}
\hline & & & & Action & Limits & & & & Dunlicate & Average & DP $\%$ & ec $\%$ & Det Limit & Count Err\% \\
\hline imple: $\quad \mathbf{R}$ & A\# & Analyte & Unit & Lower & Upper & Standard \% & Blank & $\frac{\operatorname{Result}}{1710}$ & $\begin{aligned} & \text { Duplicate } n / a \\
&\end{aligned}$ & $\frac{n / a}{\text { Average }}$ & $n / a$ & $n / a$ & $5.00 e^{-01}$ & $n / a$ \\
\hline 961002499 & & Butk Density of Sample & $\mathrm{g} / \mathrm{mL}$ & None & None & $\mathrm{n} / \mathrm{a}$ & $\frac{n / a}{n / a}$ & $\frac{1.110}{19.00}$ & $\frac{1179}{15.50}$ & 17.25 & 20.3 & $\mathrm{n} / \mathrm{a}$ & $n / a$ & n/a \\
\hline 02501 & & DSC Exotherm using Mettler & Joules/g & $\frac{-1.0 \mathrm{e}+03}{-1.0 \mathrm{e}+03}$ & $\frac{680 ; 0}{460 \% 0}$ & $\frac{101.9}{n / a}$ & $n / a$ & $\frac{19.00}{22.93}$ & 18.71 & 20.82 & 20.3 & $\mathrm{n} / \mathrm{a}$ & $\mathrm{n} / \mathrm{a}$ & $n / a$ \\
\hline & & DSC Exotherm Dry Calculated & Joutes/g oly & None & None & 98.89 & $n / a$ & 1719 & 17.11 & 17.15 & 0.41 & $n / a$ & $n / a$ & $n / a$ \\
\hline
\end{tabular}

5961002501 DSC Exache Water by TGA using Mettler
㫙T區明团

45-Day Safety Screening Report Table

$$
\text { U-102 }
$$

$\Rightarrow$ Limit violated

$\Rightarrow$ Selected Limit

is 
CORE NUMBER: 143

SEGMENT \#: $5 B$

SEGMENT PORTION: U Upper Half of Segment

\begin{tabular}{|c|c|c|c|c|c|c|c|c|c|c|c|c|c|c|}
\hline & & & & Action & Limits & & & & & & & & & \\
\hline Sample\# $R$ & A\# & Analyte & Unit & Lower & Upper & Standard \% & Blank & Result & Duplicate & Average & RPD \% & Spk Rec $\%$ & Det Limit & Count Err \\
\hline 5967002663 & & Bulk Density of Sample & g/nh & None & None & $n / a$ & $n / a$ & 1.650 & $n / a$ & $n / a$ & $n / a$ & n/a & $5.00 \mathrm{e}-01$ & $\mathrm{n} /$ \\
\hline 2665 & & DSC Exotherm using Mettler & Joules $/ g$ & $-1.0 \mathrm{e}+03$ & 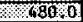 & 111.1 & $\mathrm{n} / \mathrm{a}$ & $1.94 \mathrm{e}+02$ & 98.10 & 145.9 & 65.6 & $\mathrm{n} / \mathrm{a}$ & $n / a$ & $n / a$ \\
\hline 5961002665 & & & Joules/g Dry & $-1.0 \mathrm{e}+03$ & 680.0 & $n / a$ & $n / a$ & $3.05 e+02$ & 154.4 & 229.7 & 65.6 & $n / a$ & $\mathrm{n} / \mathrm{a}$ & \\
\hline 26651 & & DSC Exotherm Dry Calculated & Joules/g Dry & $-1.0 e+03$ & masoso & $n / a$ & $\mathrm{n} / \mathrm{a}$ & $2.58 \mathrm{e}+02$ & 222.7 & 240.6 & 14.9 & $n / a$ & $\mathrm{n} / \mathrm{a}$ & n/a \\
\hline 59670026651 & & DSC Exotherm on Perk in Elmer & Joules/g & $-1.0 \mathrm{e}+03$ & 69000 & 94.94 & $\mathrm{n} / \mathrm{a}$ & $1.640+02$ & 141.5 & 152.9 & 14.9 & $\mathrm{n} / \mathrm{a}$ & $n / a$ & \\
\hline 5967002665 & & \% Water by TGA using Mettler & $\%$ & None & None & 98.89 & $n / a$ & 40.24 & 32.66 & 36.45 & 20.8 & $n / \mathbf{a}$ & $n / a$ & \\
\hline
\end{tabular}

$596 \mathrm{~T} 002665$

\begin{tabular}{|c|c|c|c|c|c|c|c|c|c|c|c|c|c|c|}
\hline & & & & Action & Limits & & & & & & & & & \\
\hline Samplen $R$ & A\# & Analyte & Unit & Lower & Upper & Standard \% & Blank & Restut & Duplicate & Average & RPD \% & pk Rec $\mathbf{z}$ & Det Limit & Count Err\% \\
\hline 5967002664 & & Butk Density of Sample & $\mathrm{g} / \mathrm{mL}$ & None & None & n/a & IV/a & 8.550 & $n / a$ & $n / a$ & $n / a$ & $n / a$ & $5.00 \mathrm{e}-01$ & $n / a$ \\
\hline 5961002666 & & DSC Exotherm using Mettler & Joules/g & $-1.0 \mathrm{e}+03$ & $480 \% 0$ & A11. & $\mathrm{n} / \mathrm{a}$ & 97.40 & 111.8 & 104.6 & 13.8 & n/a & $n / a$ & $n / a$ \\
\hline$\$ 961002666$ & & DSC Exotherm ory Calculated & Joules/g Dry & $-1.0 \mathrm{e}+03$ & $4 \mathrm{BQO}+\mathrm{O}$ & $n / a$ & ก/: & $1.76 \mathrm{e}+02$ & 201.8 & 188.8 & 13.8 & $\mathrm{n} / \mathrm{a}$ & $\mathrm{n} / \mathrm{a}$ & $n / a$ \\
\hline 5967002666 & & \% Water by IGA using Metrler & $\%$ & None & Hone & 98.89 & $n / 3$ & 46.26 & 42.96 & 44.61 & 7.40 & $n / a$ & $n / a$ & $\begin{array}{r}n / a \\
72 E+01 \\
\end{array}$ \\
\hline \$ 9610002672 & $\mathbf{F}$ & Alpha of Digested solid & uCi/g & $-1.0 e+03$ & 33.70 & 1133 & $<2.74 \mathrm{e}-03$ & $1.06 \mathrm{e}-01$ & $1.21 \mathrm{e}-01$ & $1.13 e-01$ & 13.2 & 103.3 & $7.00 e^{-03}$ & $.72 \mathrm{E}+01$ \\
\hline
\end{tabular}

\section{索 Limit violated}

$\ddot{c}$ 
CORE NUMBER: 143

SEGMENT \#: 6

SEGMENT PORTION: U Upper Half of Segment

\begin{tabular}{|c|c|c|c|c|c|c|c|c|c|c|c|c|c|c|}
\hline & & & & Action & imits & & & & & & & & & \\
\hline Sample\# & Af: & Analyte & Unit & Laver & Upper & Standard \% & Blank & Result & Duplicate & Average & RPD \% & Spk Rec \% & Det Limit & Count Err\% \\
\hline 5967002754 & & Bulk Density of Sample & $g / m L$ & None & None & $\mathrm{n} / \mathrm{a}$ & $\mathrm{n} / \mathrm{a}$ & 1.610 & $n / a$ & $n / a$ & $n / a$ & $n / a$ & $5.00 \mathrm{e}-01$ & $n / a$ \\
\hline 5967002755 & & DSC Exotherm using Mettler & Joules $/ 9$ & $-1.0 \mathrm{e}+03$ & 680.04 & 112.1 & $\mathrm{n} / \mathrm{a}$ & 70.20 & 62.20 & 66.20 & 12.1 & $n / a$ & $n / a$ & $n / \mathrm{s}$ \\
\hline$\$ 967002755$ & & DSC Exotherm Dry Calculated & Joules/g Dry & $-1.0 \mathrm{e}+03$ & 406\% & $n / a$ & $n / a$ & $1.19 \mathrm{e}+02$ & 105.8 & 112.6 & 12.1 & $n / a$ & $n / 8$ & $\mathrm{n} / \mathbf{a}$ \\
\hline 5961002755 & & \% Water by TGA using Mettler & $\%$ & Nonel & None & 98.68 & $n / a$ & 41.68 & 40.70 & 41.19 & 2.38 & $n / a$ & $n / a$ & $\mathrm{n} / \mathrm{a}$ \\
\hline
\end{tabular}

$\Rightarrow$ Limit violated

$\Rightarrow$ Selected Limit

0 
28- jun- $1996 \quad 11: 29: 27$ A- $0002 \cdot 3$

CORE MUMBER: 144

SEGMENT \#: FB

SEGMENT PORIION: Drainable Licuid

\begin{tabular}{|c|c|c|c|c|c|c|c|c|c|c|c|c|c|c|}
\hline & & & & Action & Limits & & & & & & & & & \\
\hline Sample\# & 2|A\#: & Analyte & Unit & Lower & Upper & Standard \% & Btank & Result & Dupl icate & Average & RPD \% & Spk Rec \% & Det Limit & ount Err\% \\
\hline 5967002762 & & DSC Exotherm using Mettler & Joules/g & $-1.0 \mathrm{e}+03$ & $480 \% 0$ & 110.4 & $n / a$ & $0.00 \mathrm{e}+00$ & $0.00 \mathrm{e}+00$ & $0.00 \mathrm{e}+00$ & 0.00 & $\mathrm{n} / \mathrm{a}$ & $n / a$ & $\mathrm{n} / \mathrm{a}$ \\
\hline 5967002762 & & DSC Exotherm Dry Calculated & Joules/g Dry & $-9.0 \mathrm{e}+03$ & Mado & $n / a$ & $\mathrm{n} / \mathrm{a}$ & $0.00 \mathrm{e}+00$ & $0.00 \mathrm{e}+00$ & $0.00 \mathrm{e}+00$ & 0.00 & $n / a$ & $n / a$ & $n / 0$ \\
\hline & & \% Hater by YGA using Met t ler & $\%$ & None & Hone & 98.38 & $n / a$ & 99.03 & 99.87 & 99.45 & 0.84 & $n / a$ & $\mathrm{n} / \mathrm{a}$ & $\frac{n / a}{500+0 ?}$ \\
\hline 5961002762 & & Alpha in liquid samples & $\mathrm{uCi} / \mathrm{mL}$ & $-1.0 \mathrm{e}+03$ & 60 & 103.9 & $.67 \mathrm{e}-04$ & $\angle 4.62 \mathrm{e}-06$ & $\angle 5,41 \mathrm{E}-6$ & $n / a$ & $n / a$ & 91.64 & $1.10 \mathrm{e}-05$ & $5.00 E+02$ \\
\hline
\end{tabular}

\begin{tabular}{|l|l|}
\hline 5961002762 & \%ater by YGA using Met tler \\
\hline S961002762 & Alpha in Liquid Samples
\end{tabular}

$\Rightarrow$ Limit violated

W... $\quad \Rightarrow$ Selected Limit

స్r

\section{眐TERUM}

45-Day Safety Screening Report Table 1

U-102 
CORE NUMBER: 144

SEGMENT \#: 1

SEGMENT PORTION: L LOWer Half of SEgment

\begin{tabular}{|c|c|c|c|c|c|c|c|c|c|c|c|c|c|c|}
\hline & & & & Action & Limits & & & & & & & & & \\
\hline Sample\# & A\# & Analyte & Unit & Lower & Upper & Standard $\%$ & Blank & Result & Duplicate & Average & RPD \% & Spk ReC \% & Det Limit & Count Err\% \\
\hline \$96T002551 & & Bulk Density of Sample & $\mathrm{g} / \mathrm{mL}$ & None & None & $n / a$ & $\mathrm{n} / \mathrm{a}$ & 1.670 & $\mathrm{n} / \mathrm{a}$ & $n / a$ & $n / a$ & $n / a$ & $5.00 \mathrm{e}-01$ & $n / \mathbf{a}$ \\
\hline 5961002632 & & DSC Exotherm using Mettler & Joules/g & $-1,0 \mathrm{e}+03$ & 800 & 112.5 & $\mathrm{n} / \mathrm{a}$ & 48.50 & 41.90 & 45.20 & 14.6 & $\mathrm{n} / \mathrm{a}$ & $n / a$ & $n / a$ \\
\hline 5967002632 & & DSC Exotherm Dry Calculated & Joules/g Dry & $-1.0 \mathrm{e}+03$ & $480 \ldots$ & $n / a$ & $n / a$ & 80.15 & 69.24 & 74.69 & 14.6 & $\mathrm{n} / \mathrm{a}$ & $n / a$ & $n / a$ \\
\hline 5961002632 & & \% Water by TGA using Mettler & $\%$ & None & Hone & 98.36 & $n / a$ & $40 \% 7$ & 38.20 & 39.48 & 6.51 & $n / a$ & $\mathrm{n} / \mathrm{a}$ & $n / a$ \\
\hline 5967002648 & $F$ & Alpha of oigested solid & UCi/g & $-1.0 e+03$ & 33.00 & 105.5 & $n / a$ & 6.246007 & $6.44 \mathrm{e}-01$ & $6.34 e^{-01}$ & 3.15 & 89.69 & $1.80 \mathrm{e}^{-02}$ & $1.03 \mathrm{E}+01$ \\
\hline
\end{tabular}

\begin{tabular}{|l|l|l}
5960002648 & F & Alpha of Oigested Solid \\
\hline
\end{tabular} \begin{tabular}{llllll}
$\mathrm{n} / \mathrm{a}$ & $6.24400 \mathrm{y}$ & $6.44 \mathrm{e}^{-01}$ & $6.34 \mathrm{e}^{-0}$ & 3.15 \\
\hline
\end{tabular}

\section{Drainable Liquid: Drainable Liquid}

\begin{tabular}{|c|c|c|c|c|c|c|c|c|c|c|c|c|c|c|}
\hline & & & & Action & Limits & & & & & & & & & \\
\hline Sample\# R & A\# & Analyte & Unit & Lower & Upper & Standard $\%$ & Blank & Result & Dupllate & Average & RPD \% & Spk ReC \% & Det Limit & Count Err\% \\
\hline \$96T002549 & & DSC Exotherm using Metrler & Joules/g & $-1.0 e+03$ & 460 & 110.4 & n/a & $1+12 e+0$ & 1466.6 & 129.1 & 27.2 & $\mathrm{n} / \mathrm{a}$ & $\mathrm{n} / \mathrm{a}$ & $n<a$ \\
\hline S967002549 & & DSC Exotherm Dry Calcutated & Joules/g Dry & $-1.0 \mathrm{e}+03$ & 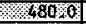 & $n / a$ & $n / a$ & $2.08 \mathrm{e}+02$ & 272.8 & 240.2 & 27.2 & $\bar{n} / \mathrm{a}$ & $n / a$ & $n / a$ \\
\hline$\$ 967002549$ & & \% Water by TGA using Mettler & $\%$ & None & None & 98,38 & $n / a$ & 52.29 & 40.23 & 46.26 & 26.1 & $n / a$ & $n / a$ & $n / a$ \\
\hline$\$ 967002549$ & & Alpha in Liquid Samples & $U C i / m L$ & $-1.0 \mathrm{e}+03$ & 64 & 712.5 & $6.43 \mathrm{e}-03$ & $2.93 \times-02$ & $4.24 \mathrm{e}^{-02}$ & $3.58 \mathrm{e}-02$ & 36.5 & 128.1 & $1.70 e^{-02}$ & $6.51 E+01$ \\
\hline
\end{tabular}

$\Rightarrow$ Limit violated

\section{¿}




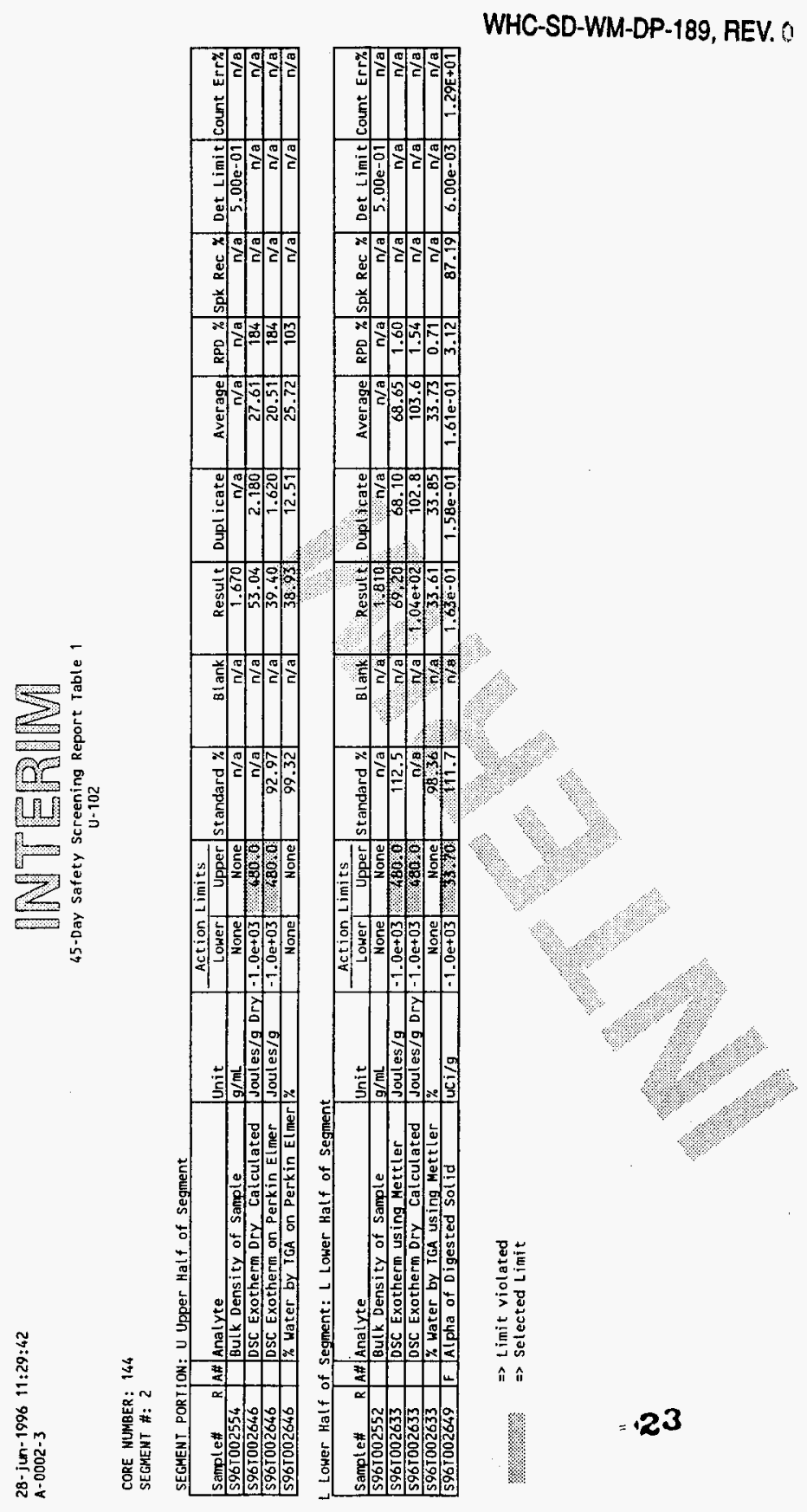




\section{CORE NUMBER: 144}

SEGHENT \#: 3

SEGMENT PORTION: U Upper half of Segment

\begin{tabular}{|c|c|c|c|c|c|c|c|c|c|c|c|c|c|c|}
\hline \multirow[b]{2}{*}{ Sample\# } & \multirow[b]{2}{*}{ A* } & \multirow[b]{2}{*}{ Analyte } & \multirow[b]{2}{*}{ Unit } & \multicolumn{2}{|c|}{ Action Limits } & \multirow[b]{2}{*}{ Standard \% } & \multirow[b]{2}{*}{ Blank } & \multirow[b]{2}{*}{ Result } & \multirow[b]{2}{*}{ Duplicate } & \multirow[b]{2}{*}{ Average } & \multirow[b]{2}{*}{ RPD \% } & \multirow[b]{2}{*}{ Spk Rec \% } & \multirow[b]{2}{*}{ Det Limit } & \multirow[b]{2}{*}{ Count Err\% } \\
\hline & & & & Lower & Upper & & & & & & & & & \\
\hline$\$ 967002555$ & & Bulk Density of Sample & g/mL & None & None & n/a & n/a & 1.710 & $n / a$ & $n / a$ & $n / a$ & $\mathrm{n} / \mathrm{a}$ & $5.00 e-01$ & $n / a$ \\
\hline 5967002647 & & DSC Exotherm using Mettler & Joules/g & $-1.0 \mathrm{e}+03$ & (68080 & 112.1 & n/a & 18.30 & 29.60 & 23.95 & 47.2 & $\mathrm{n} / \mathrm{a}$ & $n / a$ & in \\
\hline $\begin{array}{l}5961002647 \\
\$ 961002647\end{array}$ & & $\begin{array}{l}\text { DSC Exotherm Dry Calculated } \\
\text { \% Water by TGA using Metiller }\end{array}$ & $\frac{\text { Joules/g Dry }}{\%}$ & \begin{tabular}{|c|}
$-1.0 \mathrm{e}+03$ \\
Nane
\end{tabular} & None & n/a & $n / a$ & $\frac{20.13}{7.680}$ & 32.55 & 26.34 & 47.2 & $n / a$ & $n / a$ & n/ \\
\hline & & 6. Water by ligh using Mettier & 2 & & None & 98.361 & n/al & 7,680 & 10.461 & 9.070 & 30.7 & $n / a$ & $\mathrm{n} / \mathrm{a}$ & \\
\hline
\end{tabular}

\section{Lower Half of Segment: L Lower Hatf of Segment}

\begin{tabular}{|c|c|c|c|c|c|c|c|c|c|c|c|c|c|c|}
\hline & & & & Action & Limits & & & & & & & & & \\
\hline Sampie\# $R$ & A\# & Analyte & Unit & Lower & Upper & Standard \% & Blank & Result & Duplicate & Average & RPD \% & Spk Rec \% & imit & ount Err\% \\
\hline 5967002553 & & Bulk Density of Sample & $\mathrm{g} / \mathrm{mL}$ & None & None & $n / a$ & $n / a$ & 1700 & $n / a$ & $n / a$ & $n / a$ & $n / a$ & $5.00 \mathrm{e}-01$ & $\mathrm{n} / \mathrm{a}$ \\
\hline 5967002636 & & DSC Exotherm Dry Calculated & Joules/g Dry & $-1.0 \mathrm{e}+03$ & m80 0 & $n / a$ & $n / a$ & 24.07 & 29.36 & 26.71 & 19.8 & $n / a$ & $n / a$ & $\mathrm{n} / \mathrm{a}$ \\
\hline$\frac{5967002636}{5961002636}$ & & $\begin{array}{l}\text { DSC Exotherm on Perkin Elmer } \\
\text { \% Water by TGA on Perkin Elmer }\end{array}$ & \begin{tabular}{|l|l|} 
Joules $/ \mathrm{g}$ \\
$\%$
\end{tabular} & $|-1.0 \mathrm{e}+03|$ & Mone & $\frac{92,97}{90,37}$ & $n / a$ & 18.20 & 22.20 & 20.20 & 19.8 & $n / a$ & $\mathrm{n} / \mathrm{a}$ & $\mathrm{n} / \mathrm{a}$ \\
\hline 5961002662 & $F$ & Alpha of Digested Solid & $u C i / g$ & \begin{tabular}{|r|}
$-1.0 \mathrm{e}+03$ \\
\end{tabular} & None & $\frac{99.32}{102.3}$ & $\frac{\mathrm{n} / \mathrm{a}}{\mathrm{n} / \mathrm{s}}$ & $\begin{array}{r}1.1 .72 \\
1.2 \text { te-01 }\end{array}$ & $\begin{array}{r}37.05 \\
1.29 \mathrm{e}-01\end{array}$ & \begin{tabular}{r|}
24.38 \\
$1.25 \mathrm{e}-01$
\end{tabular} & $\begin{array}{r}104 \\
6.40\end{array}$ & $\begin{array}{r}n / a \\
77.44\end{array}$ & $\begin{array}{r}\mathrm{n} / \mathrm{a} \\
5.00 \mathrm{e}-03\end{array}$ & $\begin{array}{r}n / a \\
1.06 E+01\end{array}$ \\
\hline
\end{tabular}

$\Rightarrow$ Limit violated

$\Rightarrow$ Selected Limit

న్ 


\section{听T足RIM}

45-Day Safety Screening Report Table 1 U-102

CORE NUMBER: 144

SEGMENT \#: 4

SEGMENT PORTION: U Upper Half of Segment

\begin{tabular}{|c|c|c|c|c|c|c|c|c|c|c|c|c|c|c|}
\hline & & & & Action & Limits & & & & & & & & & \\
\hline Sample\# $R$ & A\# & Analyte & Unit & Lower & Upper & Standard \% & Blank & Resul $t$ & Duplicate & Average & RPD $z$ & Spk ReC \% & Det Limit & Count Err\% \\
\hline 5967002775 & & DSC Exotherm using Mettler & Joules $/ g$ & $-1.0 \mathrm{e}+03$ & 4600 & 112.1 & $n / a$ & 35.10 & 36.00 & 35.55 & 2.53 & $n / a$ & $n / a$ & $n / a$ \\
\hline 5967002775 & & DSC Exotherm Dry Calculated & Joules/g Ory & $-1.0 \mathrm{e}+03$ & YGOOO & $n / a$ & $\mathrm{n} / \mathrm{a}$ & 45.17 & 46.32 & 45.75 & 2.51 & $n / a$ & $n / a$ & $n / a$ \\
\hline S96T002775 & & $\%$ water by IGA using Mettter & $\%$ & None & None & 98.36 & $\mathrm{n} / \mathrm{a}$ & 24.47 & 20.10 & 22.29 & 19.6 & $n / a$ & $n / a$ & $\mathrm{n} / \mathrm{a}$ \\
\hline 5961002781 & & Bulk Density of Sample & $\mathrm{g} / \mathrm{mL}$ & None & None & $n / a$ & $n / a$ & 1.700 & $n / a$ & $\mathrm{n} / \mathrm{a}$ & $n / \mathbf{a}$ & $n / a$ & $5.00 \mathrm{e}-01$ & $n / a$ \\
\hline
\end{tabular}

L Lower Half of Segment: L Lower Half of Segment

\begin{tabular}{|c|c|c|c|c|c|c|c|c|c|c|c|c|c|c|}
\hline & & & & Action & Limits & & & & & & & & & \\
\hline Sample\# & \begin{tabular}{l|l|l}
$\mathbf{R}$ & A\# \\
\end{tabular} & Analyte & Unit & Lower & Upper & Standard $\mathrm{z}$ & Blank: & Result & Duplicate & Average & RPD \% & Spk Rec $\%$ & Det Limit & Count Err\% \\
\hline 5967002776 & & DSC Exotherm using Mettler & Joules/g & $-1.0 e+03$ & Fod & 114.9 & $n / a$ & 29.80 & 41.70 & 35.75 & 33.3 & $n / a$ & $n / a$ & $n / a$ \\
\hline 5967002776 & & DSC Exotherm Ory Calculated & Joules/g Dry & $-1.0 \mathbf{e}+03$ & 4 30.0 & $n / a$ & $n / a$ & 42.57 & 59.57 & 51.07 & 33.3 & $n / a$ & $n / a$ & $\mathrm{n} / \mathrm{a}$ \\
\hline 5967002776 & & $\%$ Water by IGA using Mettler & $\%$ & None & None & 99,09 & nia & 30.10 & 29.90 & 30.00 & 0.67 & $n / a$ & $n / a$ & n/a \\
\hline S961002782 & & Bulk Density of Sample & $g / m L$ & None & None & ala & $0 / \mathrm{a}$ & $\$, 610$ & $n / a$ & $n / a$ & $n / a$ & $n / a$ & $5.00 \mathrm{e}-01$ & $n / a$ \\
\hline$\$ 967002796$ & $F$ & Alpha of Digested Solid & $\mathrm{uCi} / \mathrm{g}$ & $-1.0 \mathrm{e}+03$ & Wo33 & 14.8 & $\mathrm{n} / \mathrm{a}$ & $3.45 e-01$ & $3.47 e-01$ & $3.46 \mathrm{e}-01$ & 0.58 & 99.44 & $1.30 \mathrm{e}-02$ & $1.19 E+01$ \\
\hline
\end{tabular}

W Limit violated

$\Rightarrow$ Selected Limit

थู 
WHC-SD-WM-DP-189, REV. 0

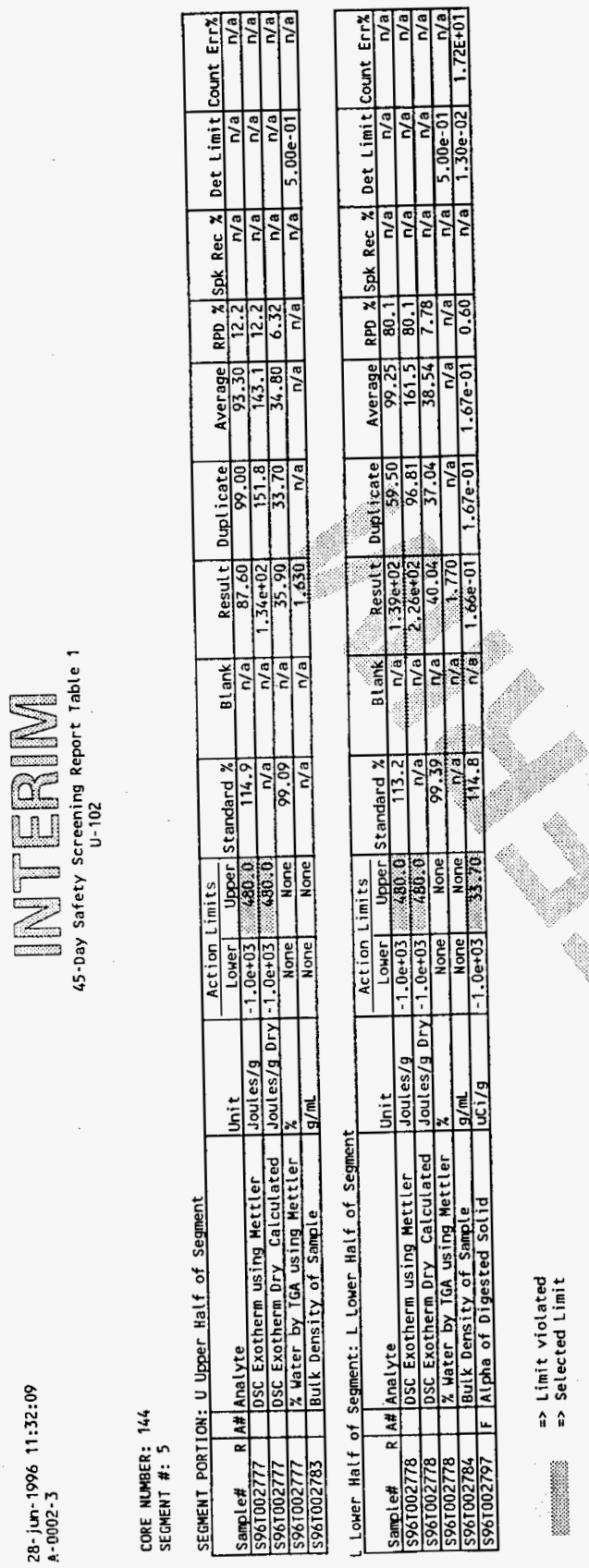


CORE NUMBER: 144

SEGMENT \#: 6

SEGMENT PORIION: U Upper Half of Segment

\begin{tabular}{|c|c|c|c|c|c|c|c|c|c|c|c|c|c|c|}
\hline \multirow{2}{*}{ Sample\# R } & \multirow[b]{2}{*}{ A\# } & \multirow{2}{*}{ Analyte } & \multirow[b]{2}{*}{ Unit } & \multicolumn{2}{|c|}{ Action Limits } & \multirow[b]{2}{*}{ Standard $\%$} & \multirow[b]{2}{*}{ B L ank } & \multirow[b]{2}{*}{ Result } & \multirow[b]{2}{*}{ Duplicate } & \multirow[b]{2}{*}{ Average } & \multirow[b]{2}{*}{ RPD \% } & \multirow[b]{2}{*}{ Spk Rec \% } & \multirow[b]{2}{*}{ Det Limit } & \multirow{2}{*}{ Count Err\% } \\
\hline & & & & Lower & Upper & & & & & & & & & \\
\hline$\frac{\text { 596T002779 }}{\text { S96T002779 }}$ & & DSC Exotherm using Mettler & Joules/g & $-1.0 \mathrm{e}+03$ & 48099 & 113.2 & $\mathrm{n} / \mathrm{a}$ & 63.20 & 76.60 & 69.90 & 19.2 & $n / a$ & $\pi / a$ & n/a \\
\hline$\frac{596 T 002779}{\text { S96T002779 }}$ & & DSC Exotherm Dry Calculated & Joules/g Dry & $-1.0 \mathrm{e}+03$ & $680 \%$ & $n / a$ & $n / a$ & $1.34 \mathrm{e}+02$ & 162.2 & 148.0 & 19.2 & $n / a$ & $n / a$ & $n / a$ \\
\hline$\frac{1596002718}{5961002785}$ & & $\begin{array}{l}\text { \% Water by IGA using Mettler } \\
\text { Bulk Density of Sample }\end{array}$ & $\frac{\%}{0 / m L}$ & None & None & 99.39 & $n / a$ & 50.10 & 55.46 & 52.78 & 10.2 & $n / a$ & $n / a$ & $n / a$ \\
\hline & & & & None ] & None & & $\mathrm{n} / \mathrm{a}$ & 1,610 & $n / a$ & $n / a$ & $n / a$ & $n / a$ & $5.00 e-01$ & $n / a$ \\
\hline
\end{tabular}

L Lower Half of Segment: L Lower Half of Segment

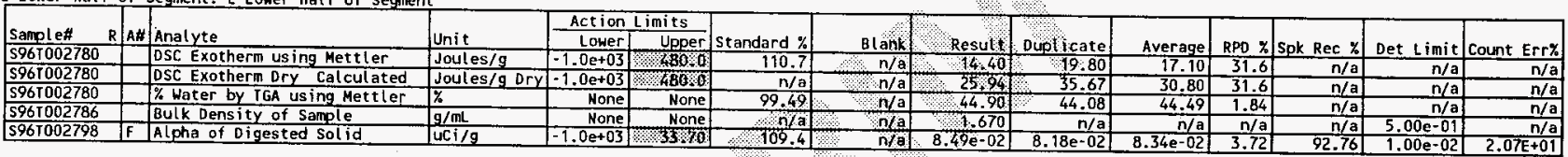

Fin..... $\Rightarrow$ Limit violated

$\Rightarrow$ Selected Limit

ปู 
WHC-SD-WM-DP-189, REV. 0

\section{ATTACHMENT 1}

\section{U-102 PUSH MODE CORE SAMPLE BREAKDOWNS}


WHC-SD-WM-DP-189, REV. 0

THIS PAGE. WAS INTENTIONALLY LEFT BLANK 


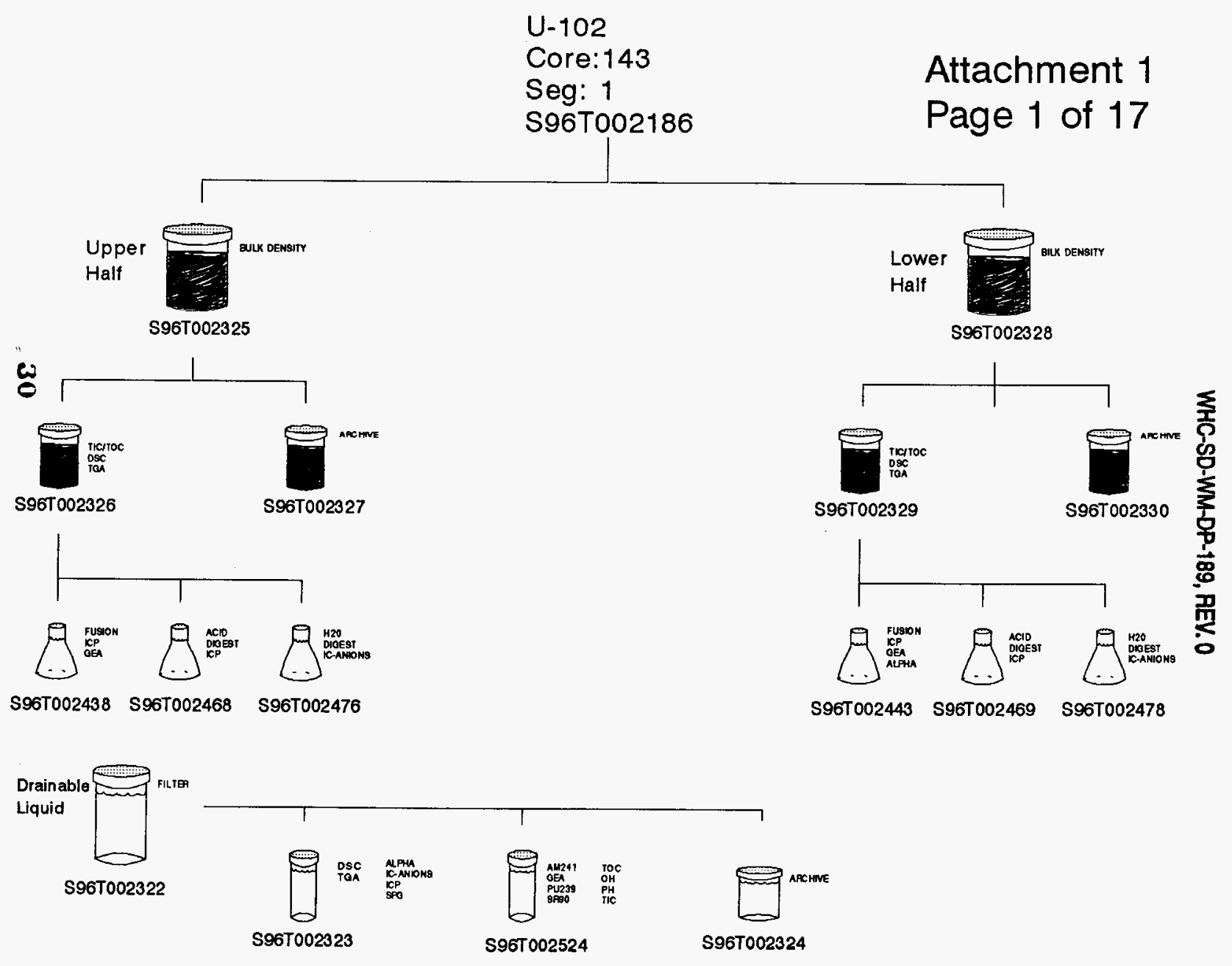




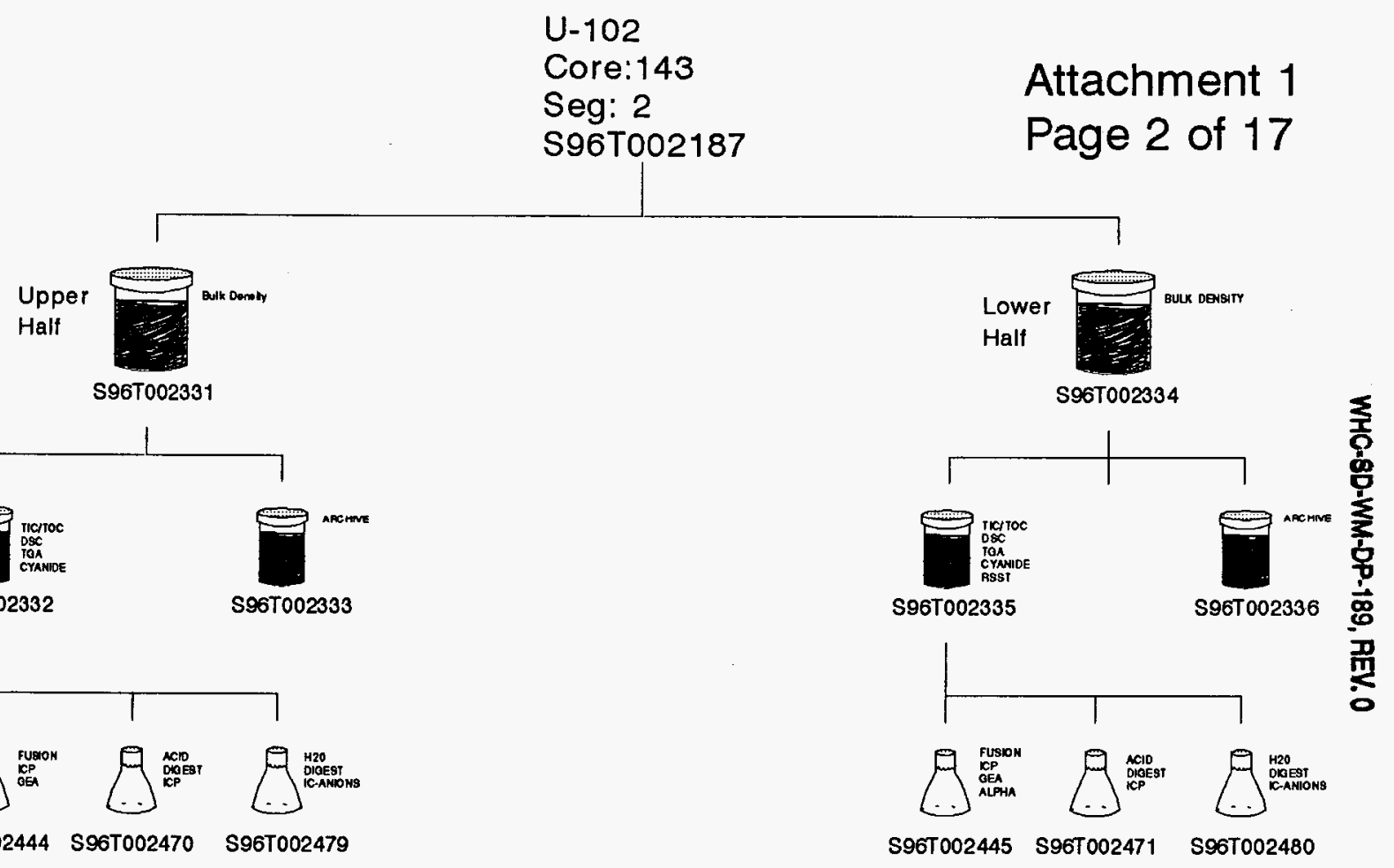




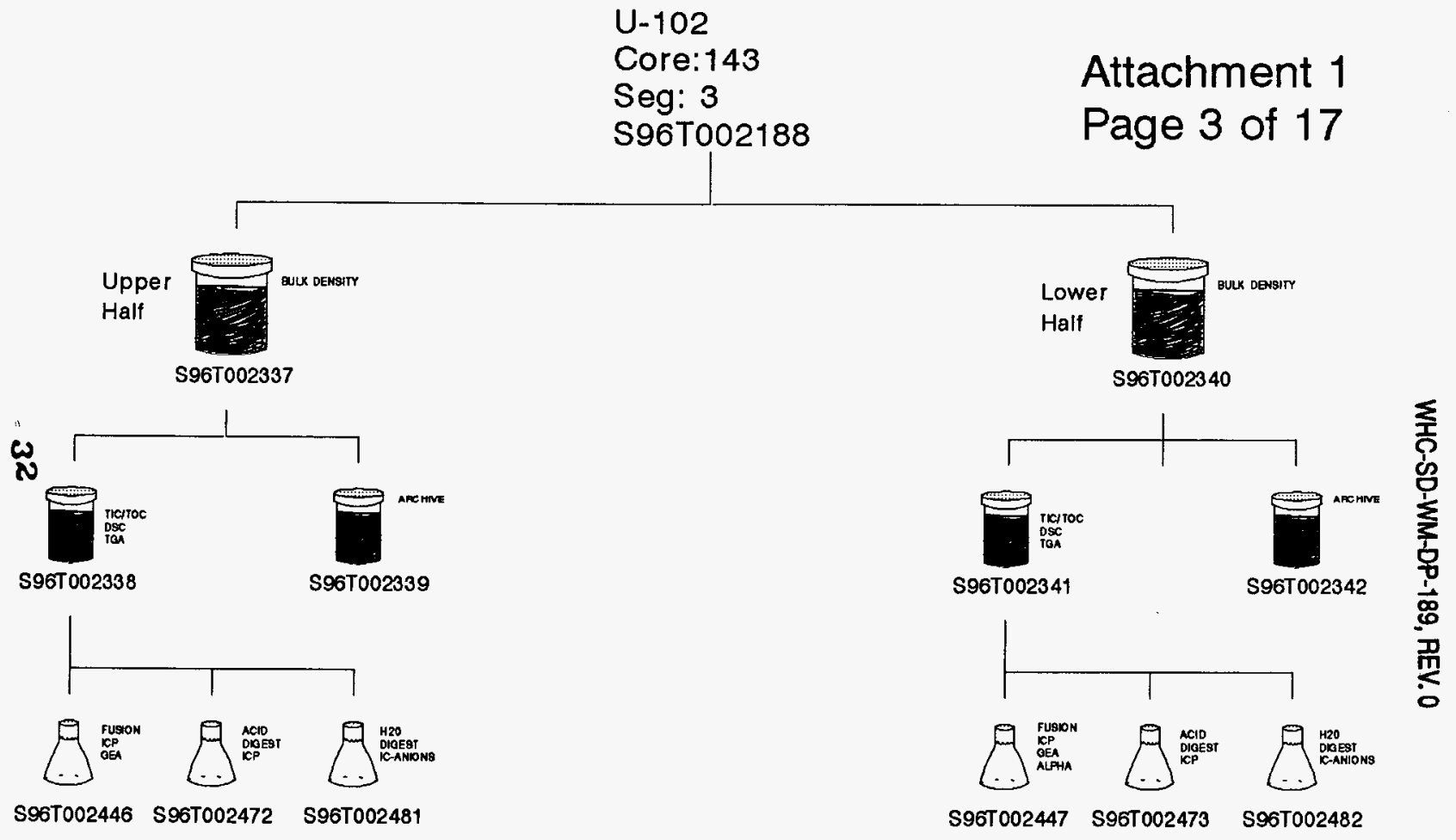


U-102

Core: 143

Seg: 4

S96T002189

\section{Attachment 1 Page 4 of 17}

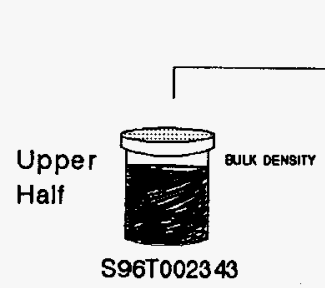

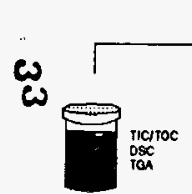

S96T002344
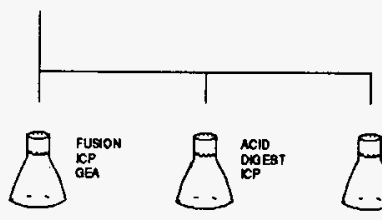

S96T002448 S96T002474 S96T002483
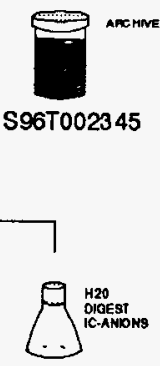

S96T002345

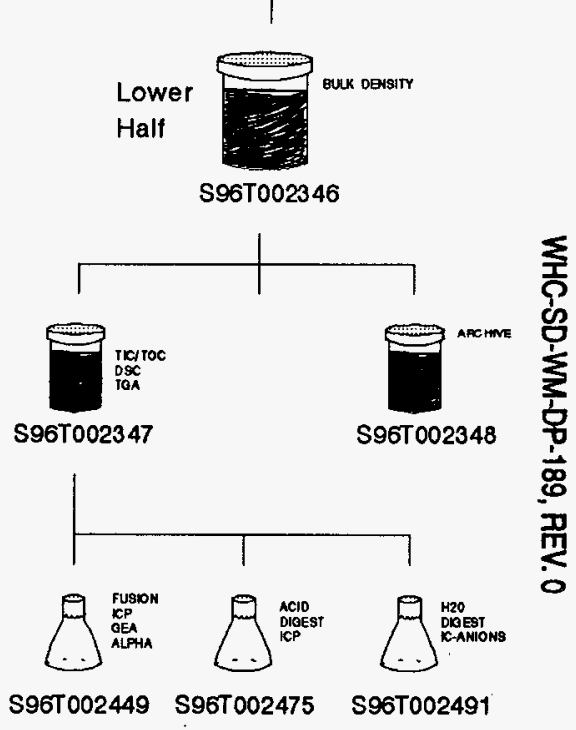


U-102

Core:143

Seg: 5

S96T002190

\section{Attachment 1 Page 5 of 17}

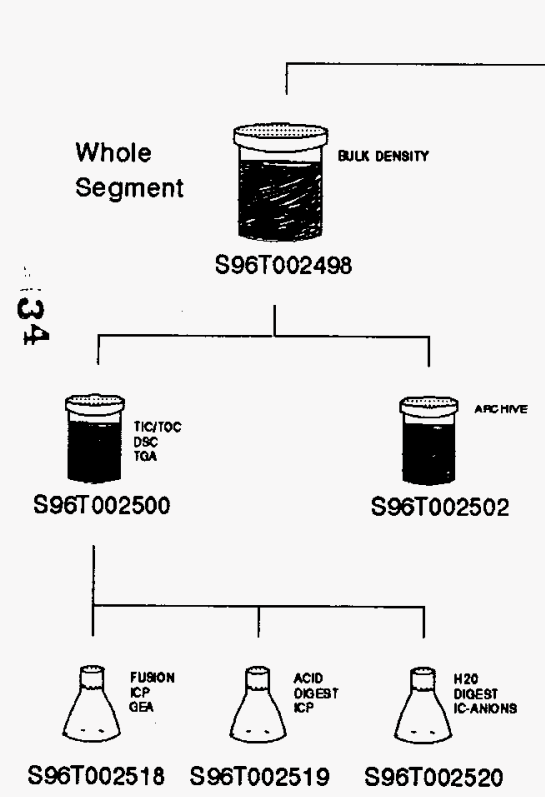

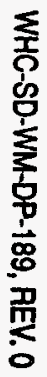


U-102

Core: 143

Seg: $5 \mathrm{~A}$

S96T002191

\section{Attachment 1 Page 6 of 17}
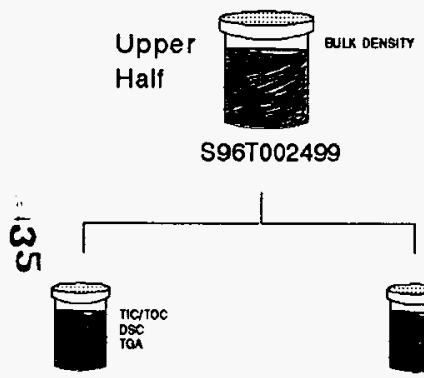

S96T002501

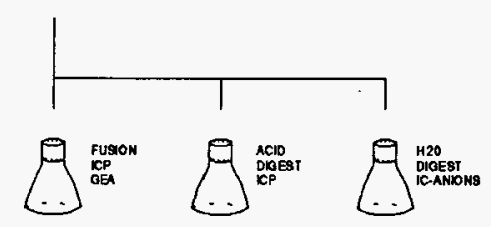

S96T002521 S96T002522 S96T002523 
U-102

Core:143

Seg: $5 B$

S96T002509

\section{Attachment 1 Page 7 of 17}
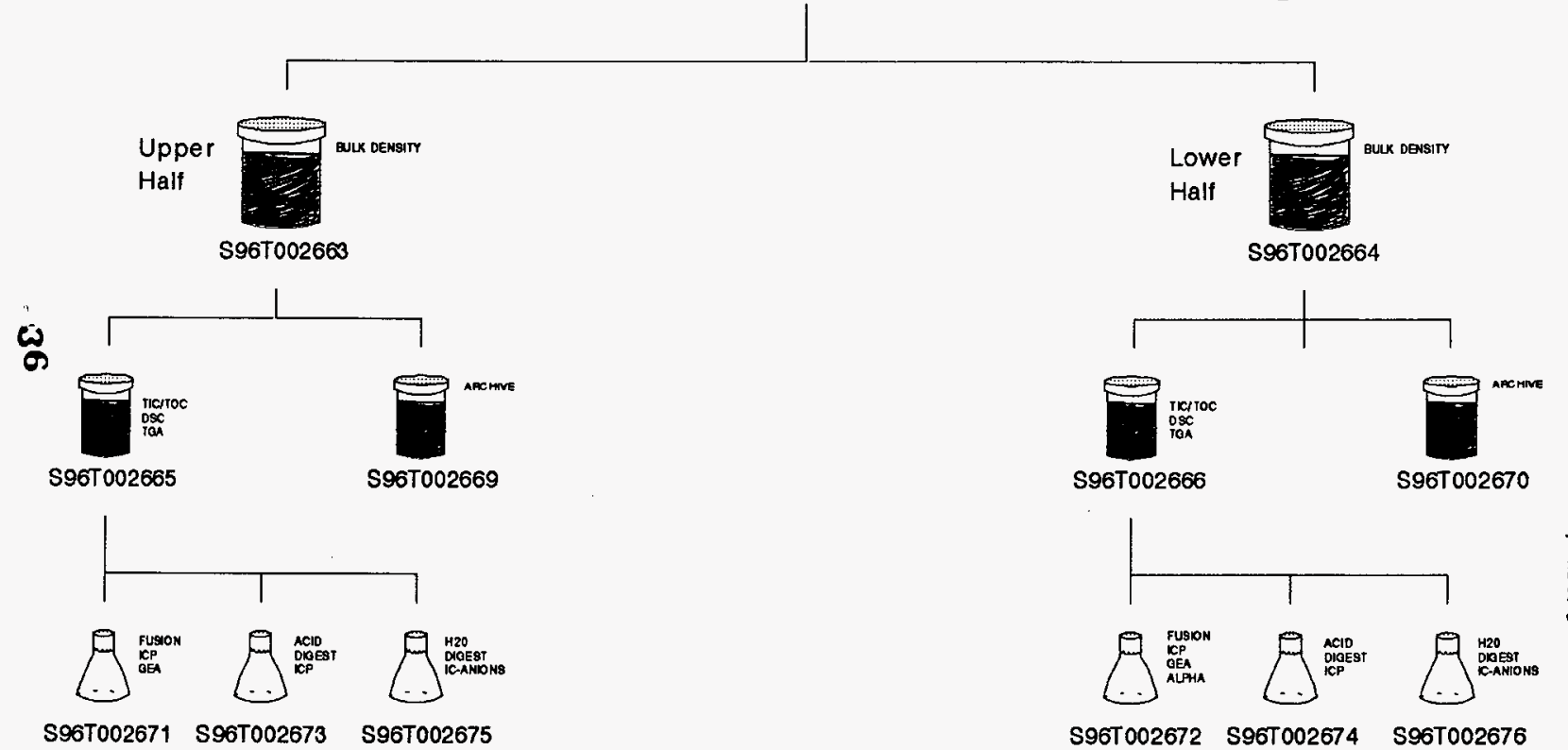


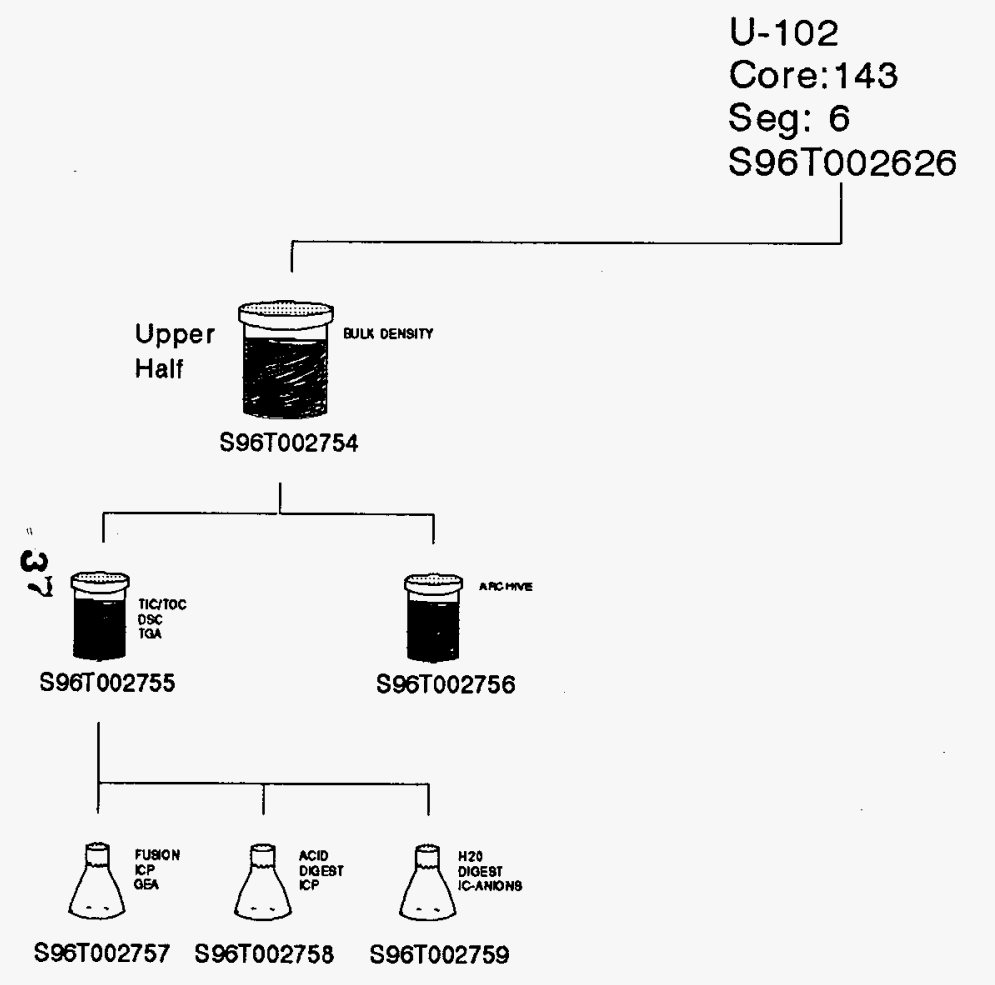

Attachment 1 Page 8 of 17

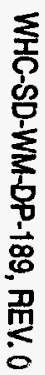

S96T002757 S96T002758 S96T002759 

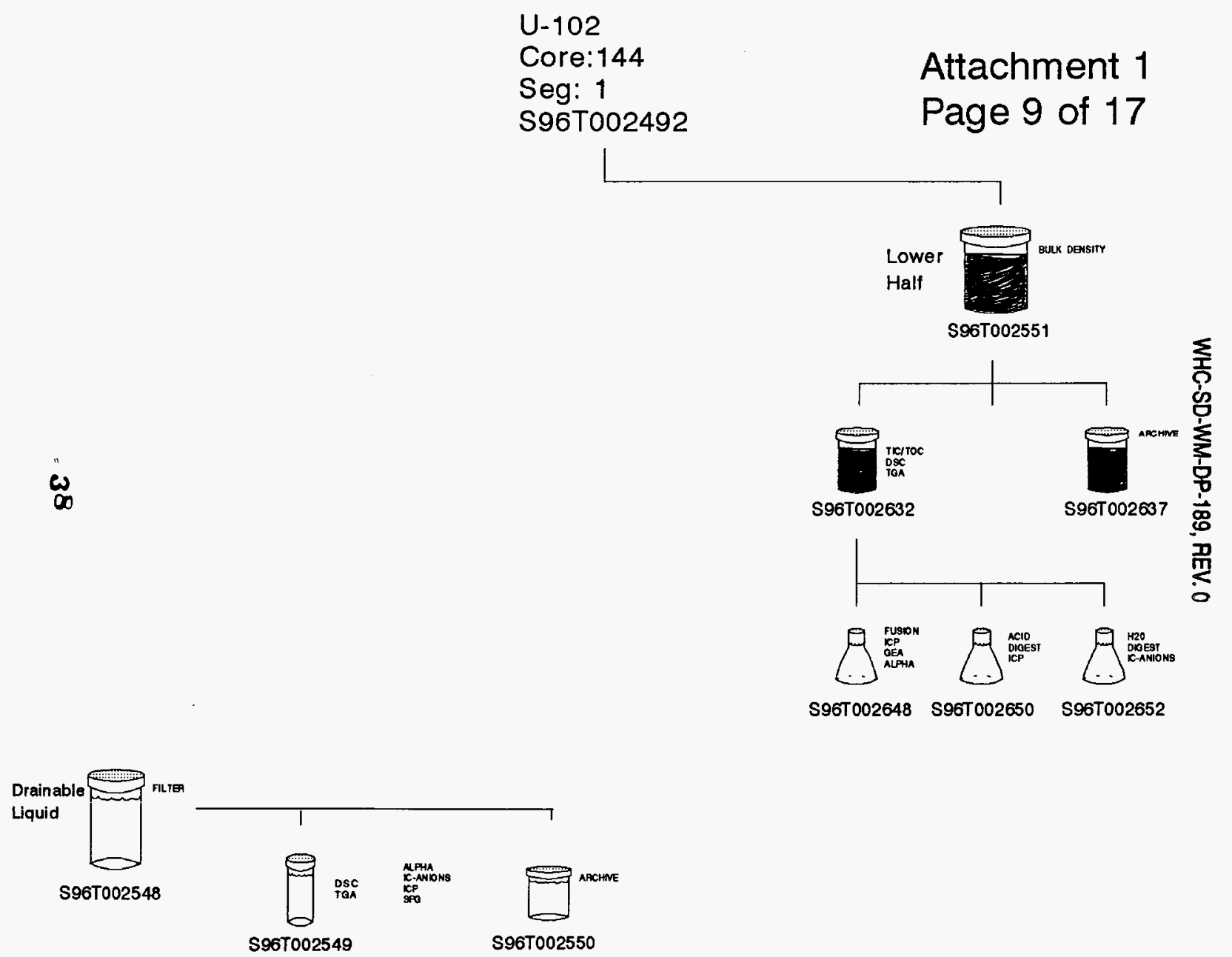
U-102

Core: 144

Seg: 2

S96T002493

\section{Attachment 1 Page 10 of 17}

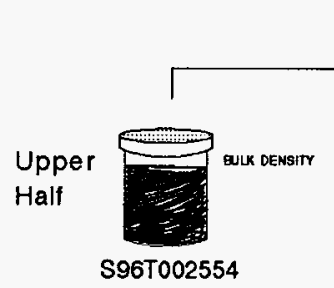

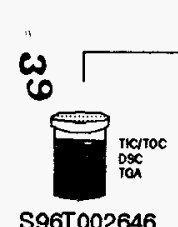

S96T 002646
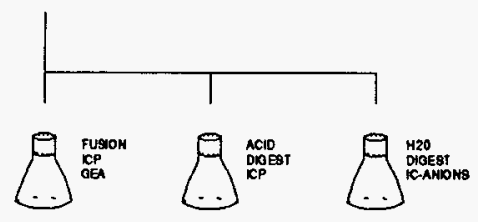

S96T002654 S96T002656 S96T002659

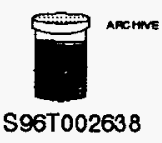

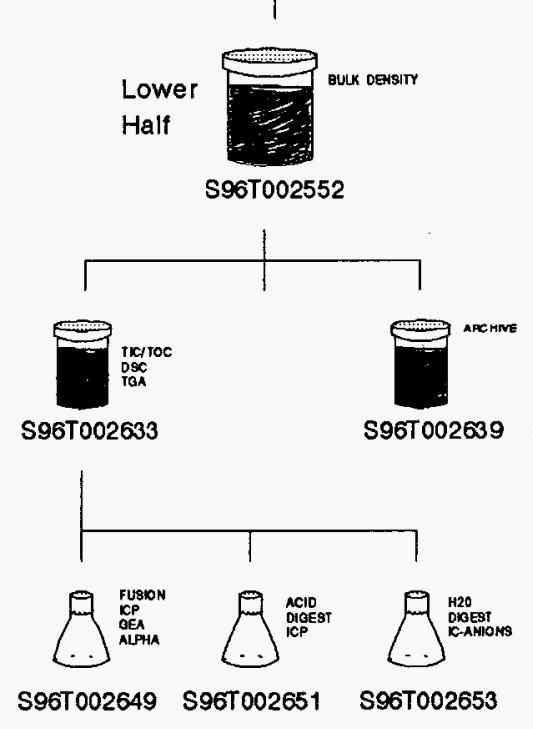




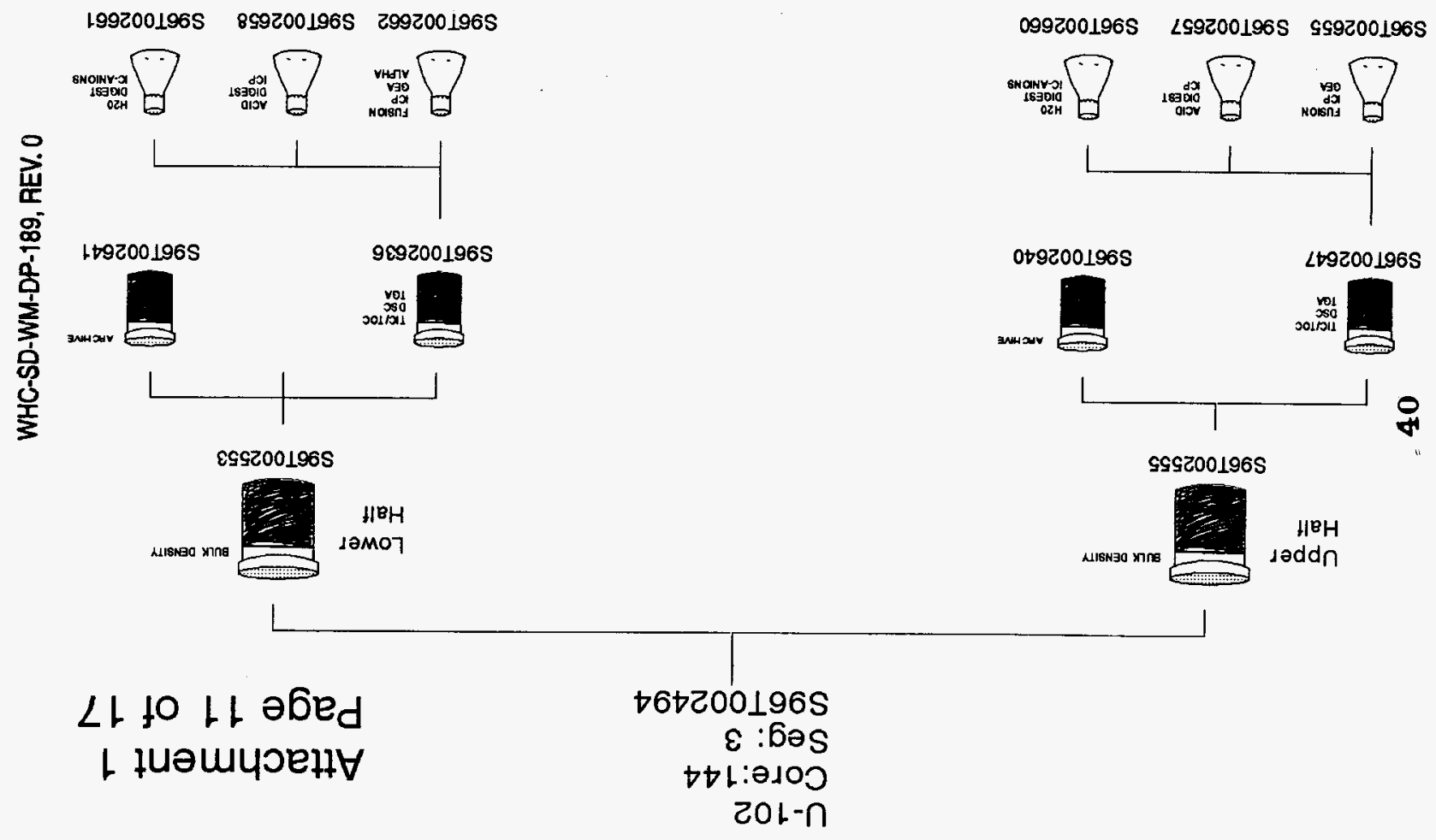


U-102

Core: 144

Seg: 4

S96T002628

\section{Attachment 1 Page 12 of 17}
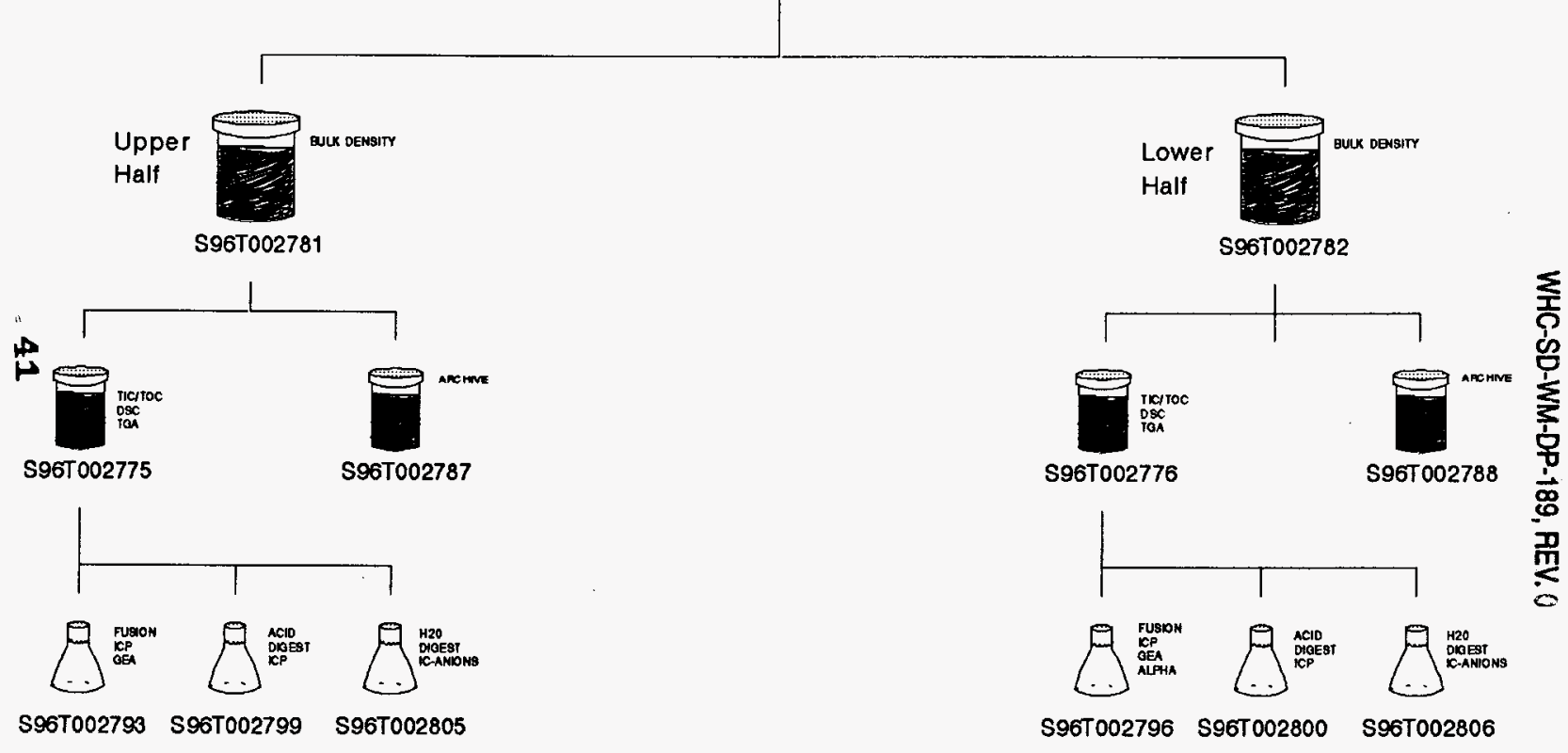
U-102

Core:144

Seg: 5

S96T002629

\section{Attachment 1 \\ Page 13 of 17}

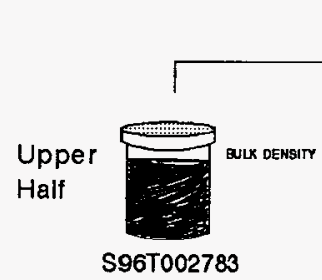

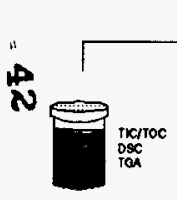

S96T002777

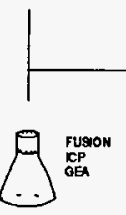

S96T002794 S96T002801
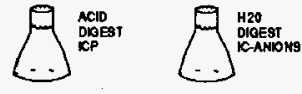

S96T002807

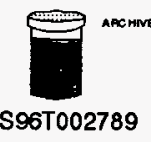

S96T002789 
U-102

Core:144

Seg: 6

S96T002630

\section{Attachment 1 Page 14 of 17}
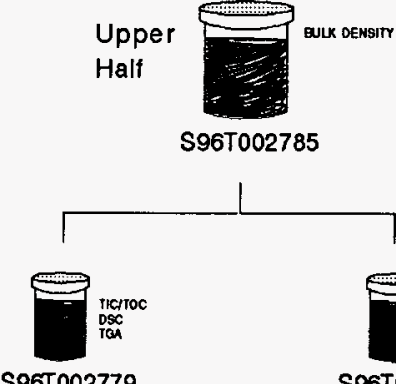

S96T002779

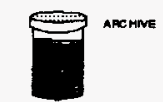

S96T002791

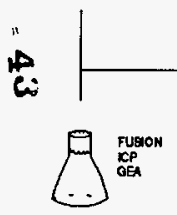

S96T002795 S96T002803

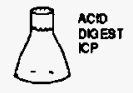

...

S96T002809

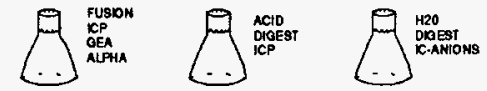

S96T002798 S96T002804 S96T002810 
U-102

Core:144

Seg: $6 \mathrm{~A}$

S96T002510

\section{Attachment 1 \\ Page 15 of 17}

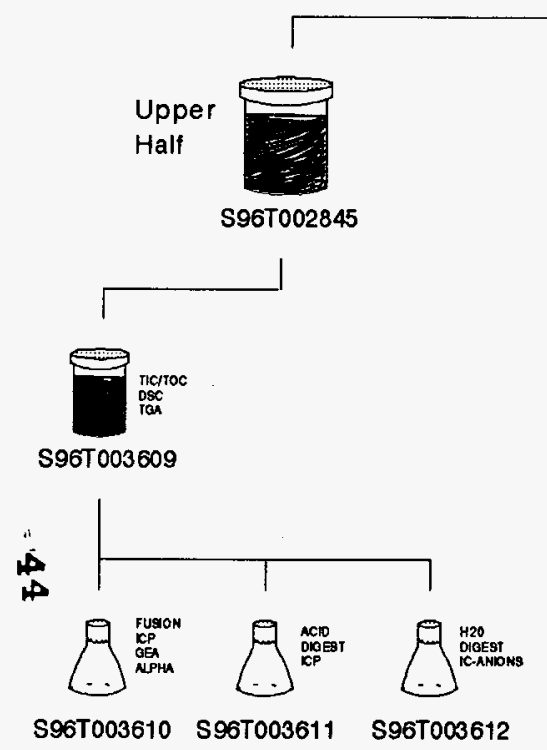


U-102

Core:144

Seg: FB

S96T002631
Attachment 1 Page 16 of 17

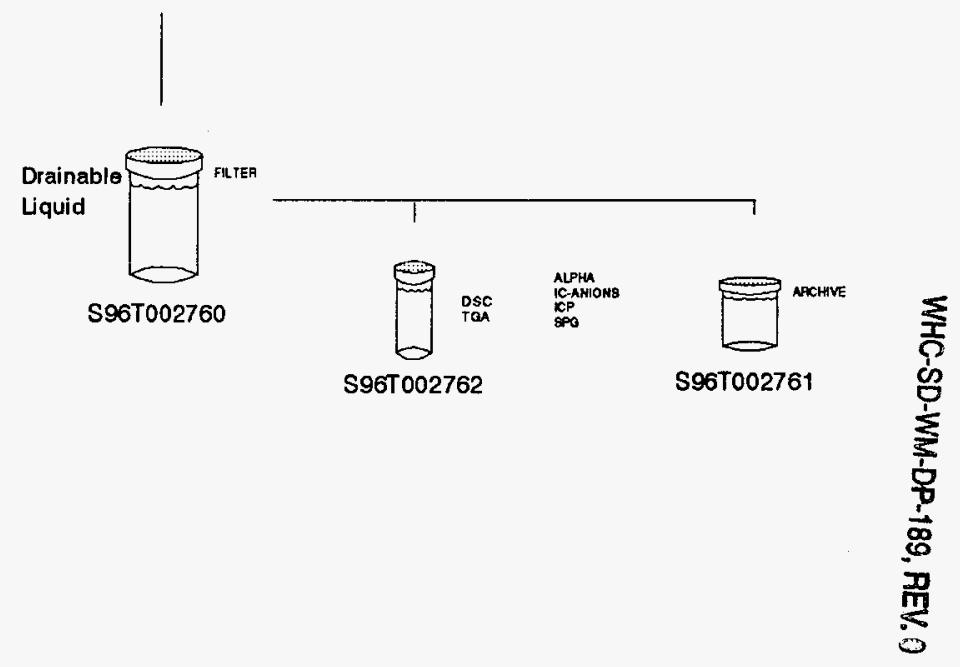


U-102

Core:144

Seg: $\mathrm{LiBr}$

S96T002511
Attachment 1

Page 17 of 17

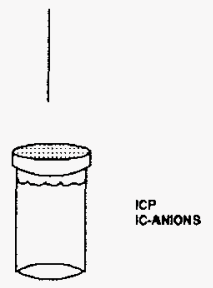

is.

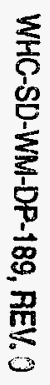


WHC-SD-WM-DP-189, REV. 0

CHAIN OF CUSTODY FORMS

. .47 
WHC-SD-WH-OP-189, REV. O 


\section{CHAIN-OF-CL!STODY RECORD FOR COAE SAMPLING}

(1) Shipment Number $\mathrm{MO}(1.08$.TF (2) sample Number $76-182$ (3) Supe visor

(6) 5 ogment

(7) Cor 143

(4) Tonk

Odiacion Survery Data:
Over Top Dose Rate

Side Dose Rate

$\frac{2.5 h+2 h}{150 / 6 n}$

(33) LABORATORY

A) Stipment Description
A. Work Package Number
B. Cosk Seal Number
C. Sampler Seriat Number
D. Date and Timo Sampler Unsoated
E. Expected Liquid Content.
F. Expocted Solid Content
G. Dose Rate Through Drill String
A. Expected Sample Length

$\frac{5+040}{1055}$

$\omega 5-96-00058 / 0$

$22 \mathrm{math}$

Bottom Dose Rate

$8 \mathrm{mat} / \mathrm{t}$

$-6220 \mu M$

Smearable Contamination

(ylghal

- 1 (Alpha)

RCT -

$2 \operatorname{leg} \sqrt{\log \cos ^{2}}$

$\leq 1600 / D P m$

AC

joofo.Gammal

cr. 120120

(1) INFORNATION flnckude statement of laboratory tests to be performed.)

苋

$\stackrel{m}{\ddot{m}}$

(12) Field Cormonis

15

$\ddot{m}$

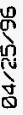
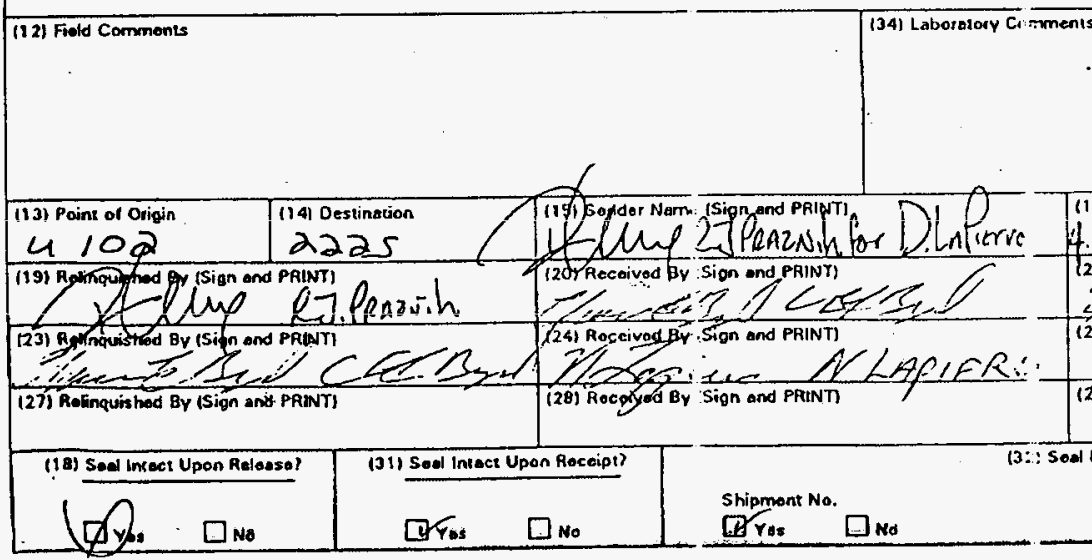

-

11426

95-3105

$4-16-96 \quad 0137$

$10 \%$

907

$\frac{1.2 R / 48}{1911}$ $19^{\prime \prime}$

) 


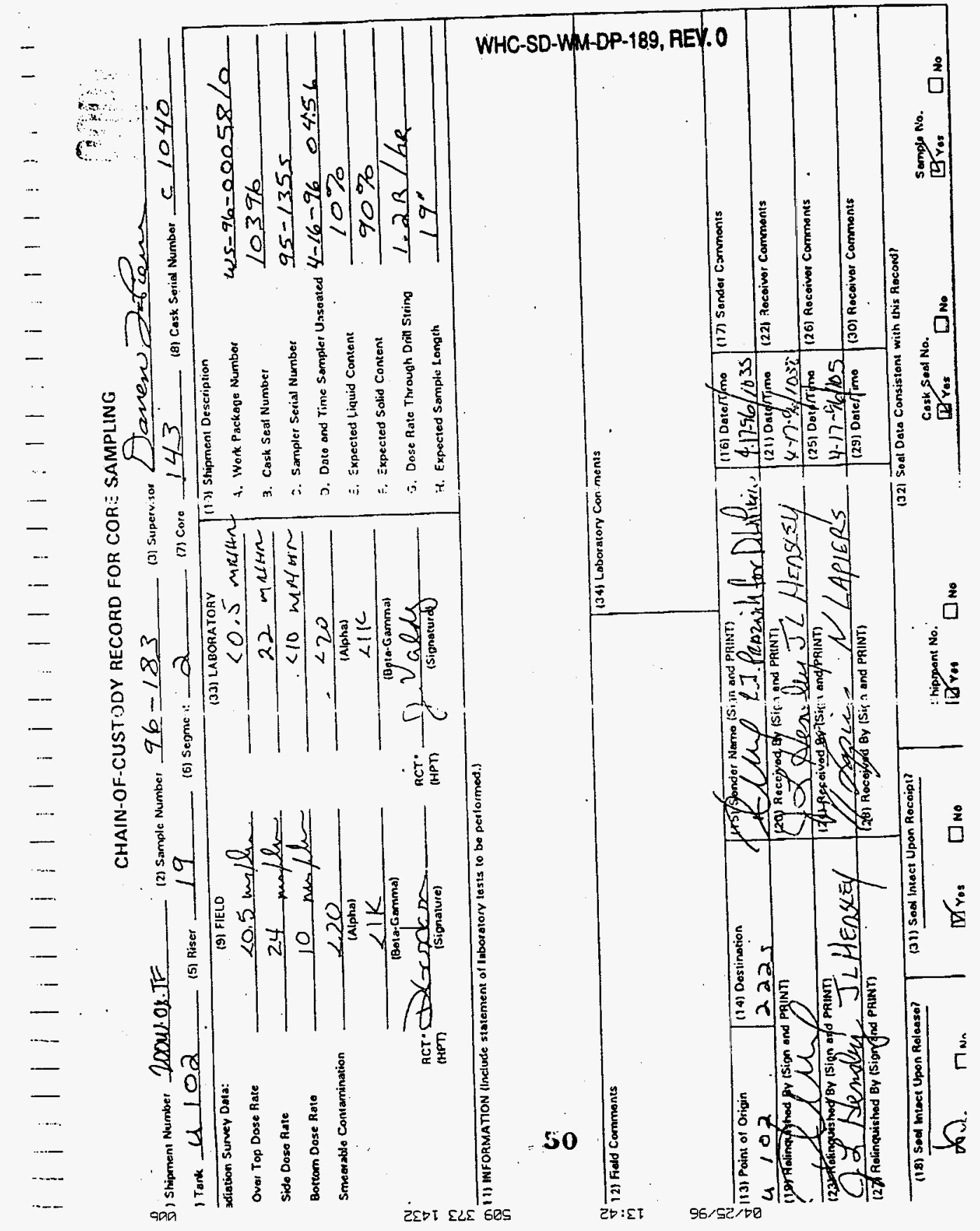




\section{CHAIN-OF-CUSTOOY RECORD FOR COFE SAMPLING}

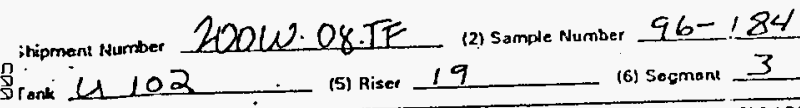
(5) Riser -19

iation Surver Data:

wer Top Dose Rate

ide Dose Rate

cottom Dose Rate

imosablo Comamination

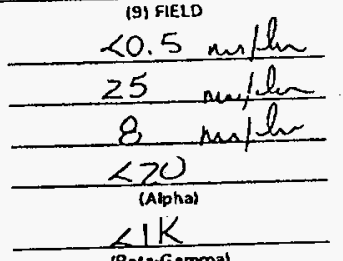

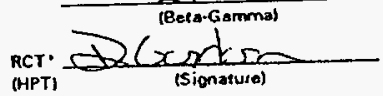

(3) Super isor $Y$ acese (7) Core 143
(9) Cosk Soriel Numbor $C / 03$

$\underset{\sim}{m}$

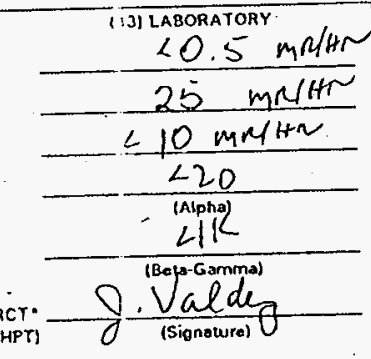

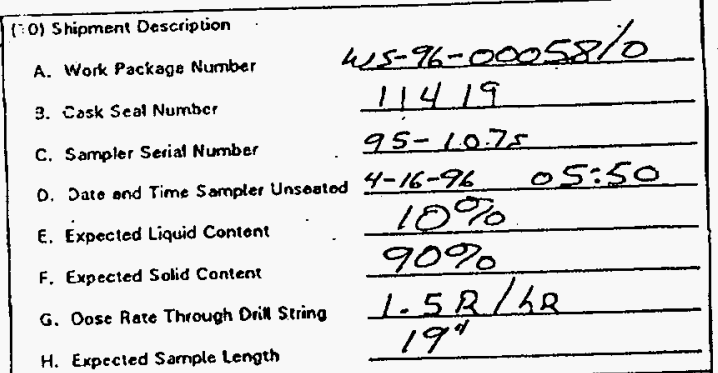

M I) INFORMATION (Include statement of taborslory tests to be perloumed.

品

2) Field Commonts

min

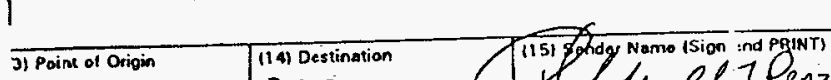

Jir 2225

बi grtinquis) By (Sign and PRINT)

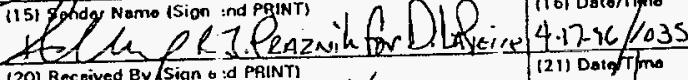
(34) Laboratory Co: mants

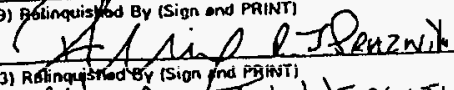

\section{(17) Sonder Comments}

$\mu \times 7.46,1052$

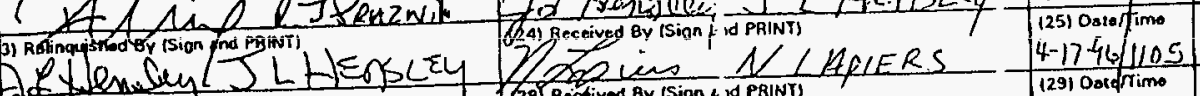
Ff relinquished By (Sigp and PRINT)

\begin{tabular}{|c|c|}
\hline (IB) Sodl Intoct Upon Roleoso? & (31) Soed Insact Upon Receipl? \\
\hline QNo & Dfros \\
\hline
\end{tabular}
(28) Ragetived By (Sion \& id PRINT) (29) Oatefrime

(30) Rocaiver Commonts

(32 Soal Date Consisiont with this. Record? 
CHAIN-OF-CUS TODY RECORD FOR CORE SAMPLING

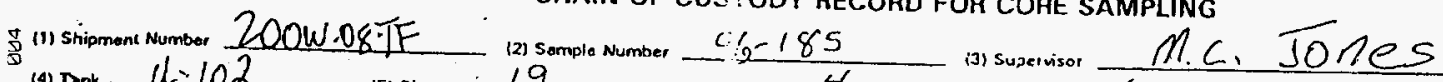
(4) ronk- $1=102$ (5) Riser 19 (6) Seg- =nt 4

Padiation Surver Data: (9) FIELD

Over Top Doso Rase

Side Oose Rate

Botiom Dose Rate

Smarablo Conternination

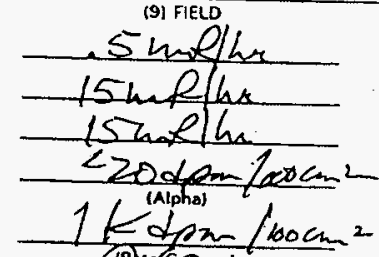

iT: 0 ana 143

(8) cask sorial Numbar ca 105/2.
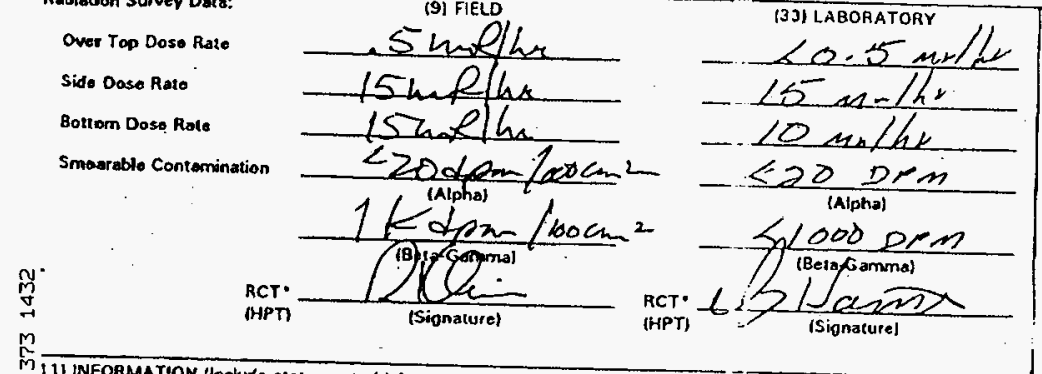

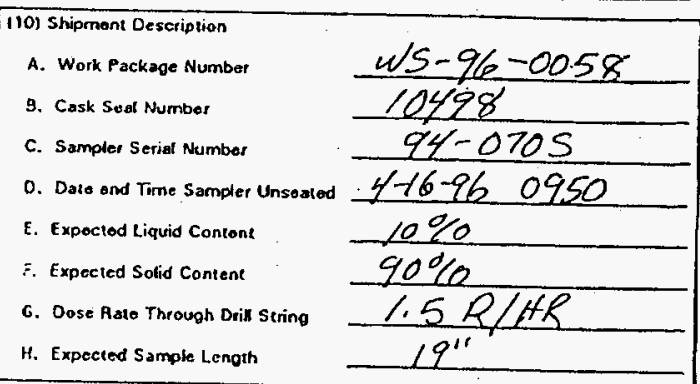

$\stackrel{9}{8}$

y

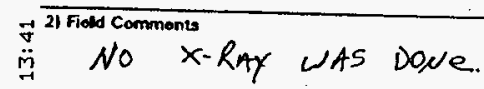

\%ั

กู่ 3 Polmt of Oigin के $t$ th -10 2 2

आ) Roligenizhod By ision on $22-5$

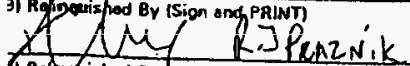

Tireconsuishod By (sion apd PRINT)

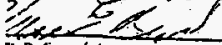
$C 2+5$

sts 10 be pertormed.]
A. Work Package Number
9. Cask Sual Number
C. Samplet Seriat Number
Expociod hiquid Consone
Expected Solid Content
G. Dose Rate Through Drill String
H. Expected Sampla Lenoth

.

$-10 \%$

7 Rolinquished By (Sion and PRINT

(18) Seal Intect Upan Rolasso?

Q Vos $\square$ No
(31) Soal Intoct Upon Receipt? Drane. Пu.
(34) Cobora:ory Commenis.

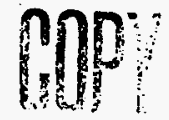

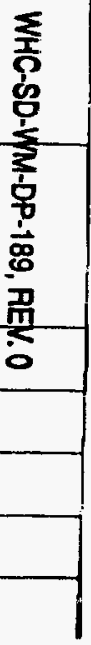




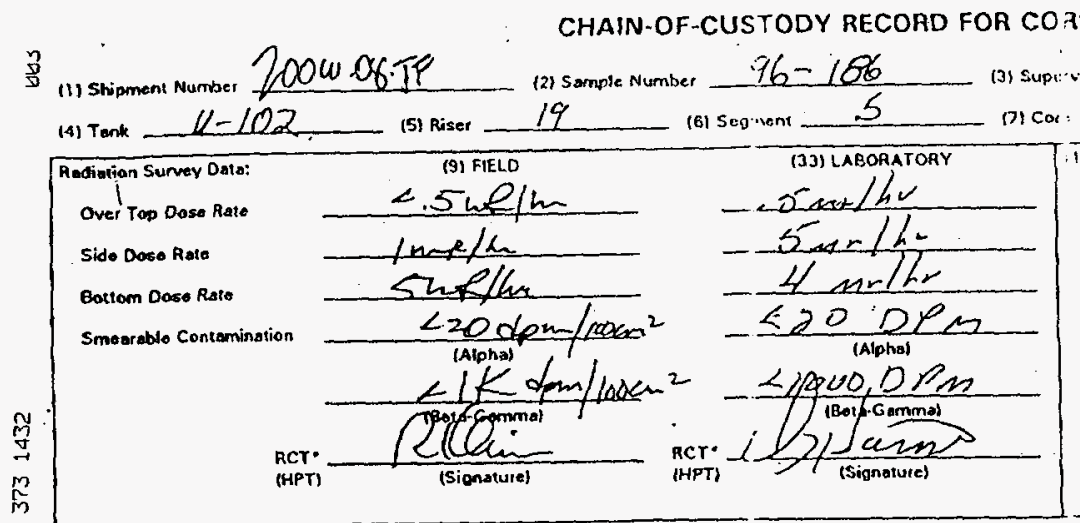

\section{SAMPLING}

Alac. Jones

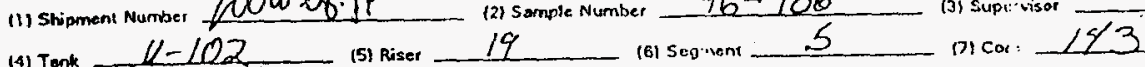

(8) Cosk Sarial Number 2004

兒

(1) iNFORMATION IInciude statement of loberatory cests 10 bo performed.)

ย

0) 5 ripment Descoiption

A. Work Pack ago Numbor

uls-25-0058

B. Sask Seal Number 10497

C. Sompler Serist Number

$195-0685$

o. Oats and Time Sampler Unseated titi $4-16-961245$

E. Experted Linuid Conton

$5 \%$

E. Expected Lipina Contone

F. Expected Solid Content

G. Dose Rate Through Drill String

H. Expocted Sample Length

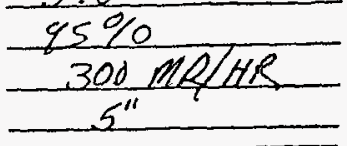
112) Fiold Comments

SAMpl- was anly pushed $5^{\prime \prime} \therefore 5$ high Down force.

岕

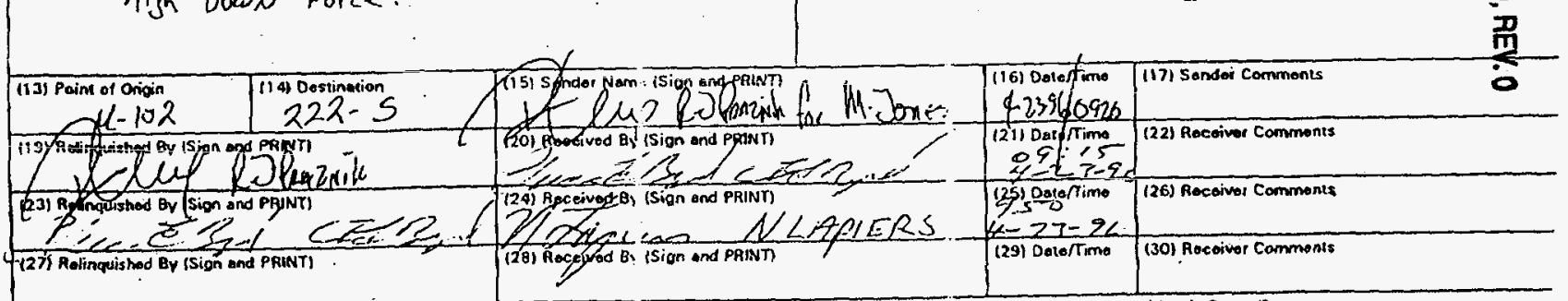

Shipnent No.

Thipnent No. $\square$ No
13.) Soal Date Consistont with this Roeord?

Cask Soot No.

Qures DNo
Sample No.

Dres $\square$ no 


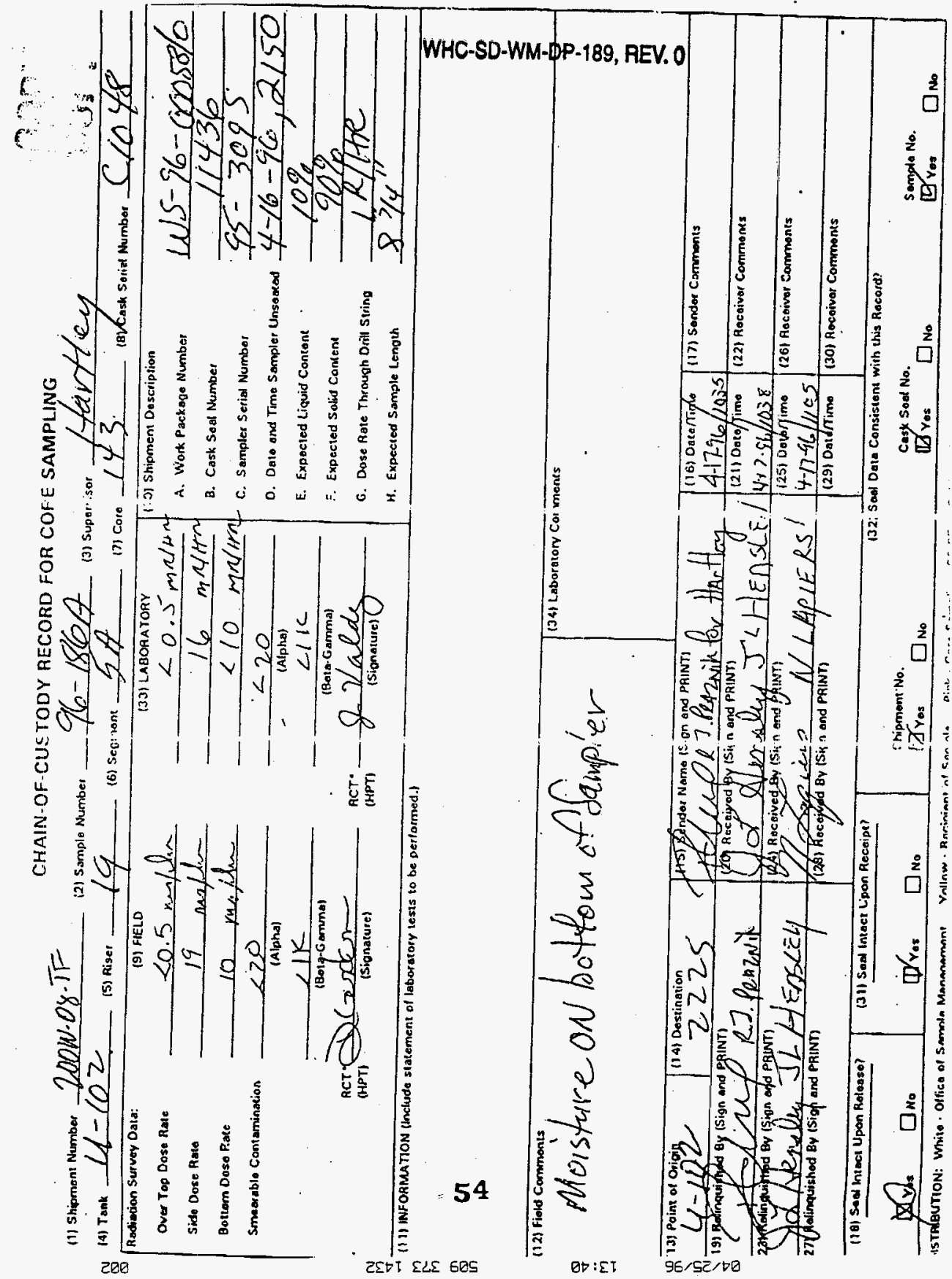




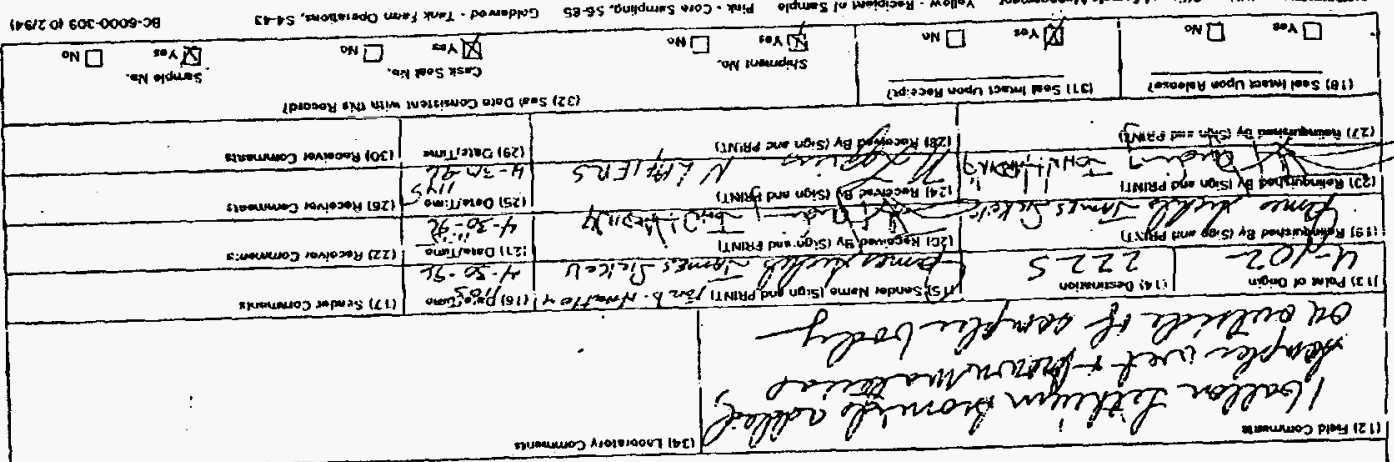

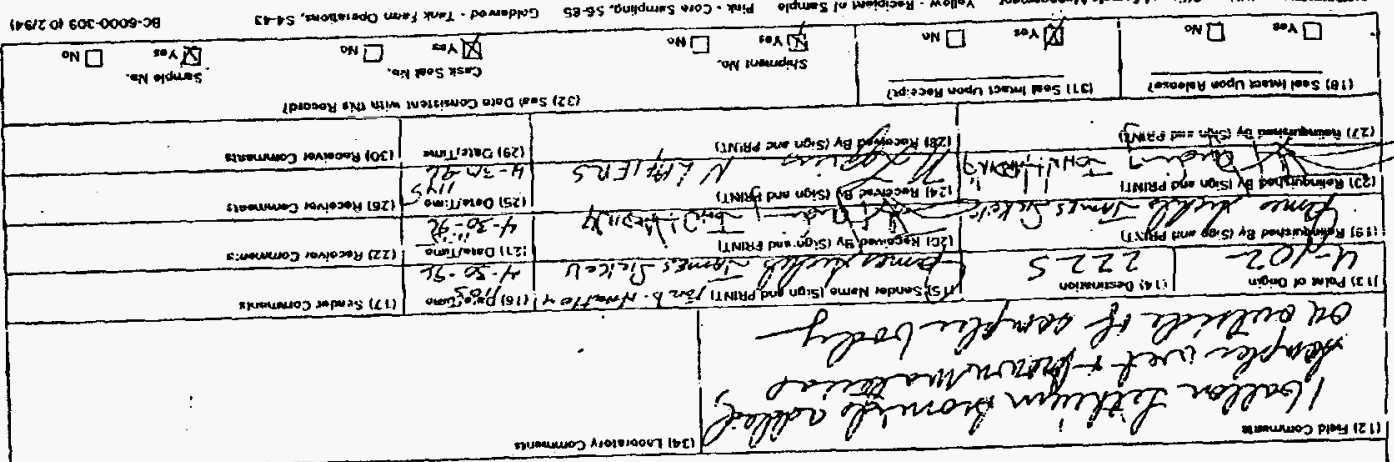

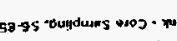

is

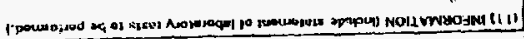

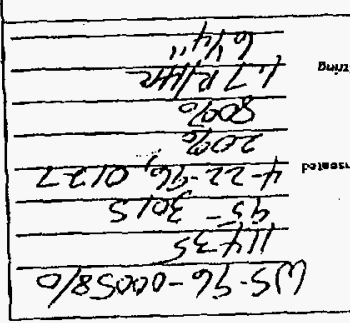

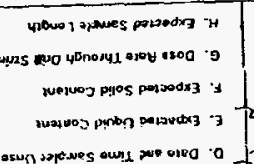
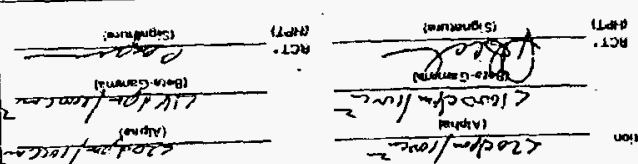

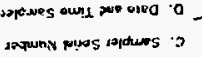

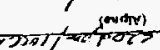

aquin pos ystro o

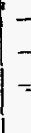

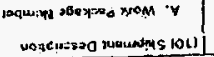

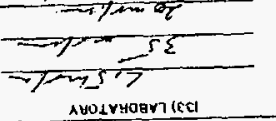

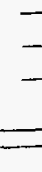

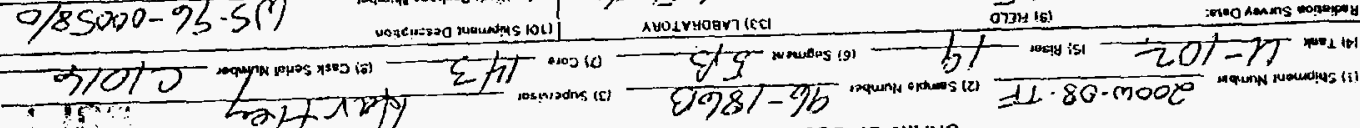

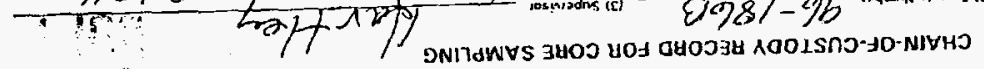

$\therefore$ an 


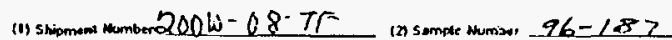

(4) Tank $4-102$ 19

(3) superixion Asunen

(i) Core 143. (9) Cast seriet Humbar _ $C-1023$

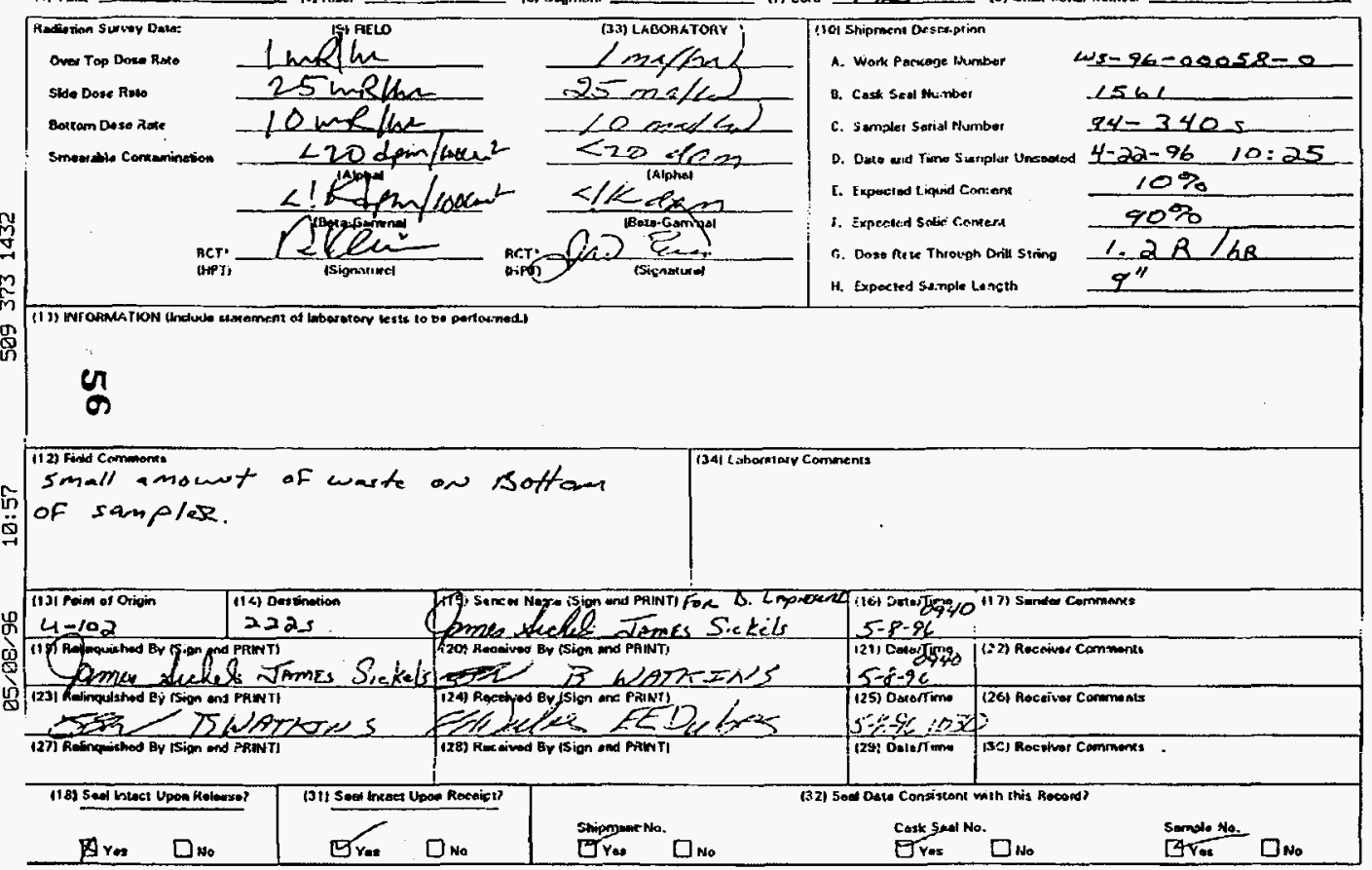

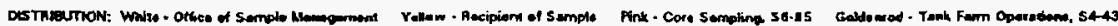




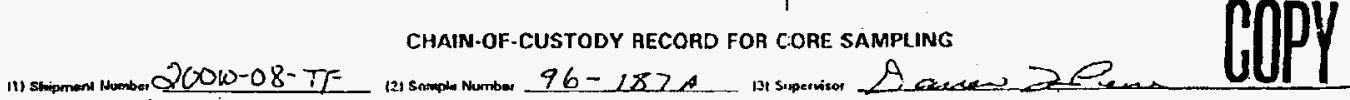

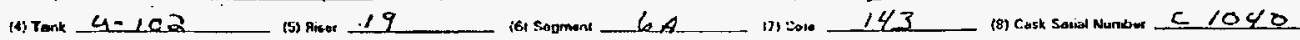
ira shiprnent De scripion

\begin{tabular}{|c|}
\hline \\
\hline \\
\hline \\
\hline 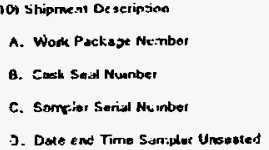 \\
\hline :- Espocted tiquid Contunt \\
\hline F. Expectes soxis Comant \\
\hline B. Dose Rale Through Ond Stirio \\
\hline H. Expected Sumple Length \\
\hline
\end{tabular}

$\operatorname{mes}-26-\infty 58 / 0$ 1548 $95-304$

$5 \log 606 x$ $\frac{e}{a}$ $2 \infty 0 M R / G R$ $\Omega$

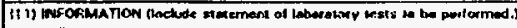

\section{$\sqrt{1}$}

璐

亶

穴

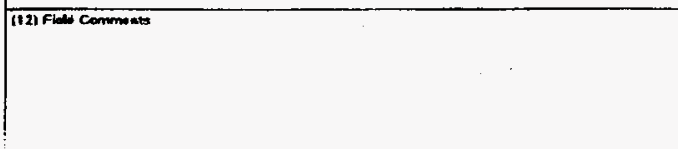
(34) Labores:uny Cowements

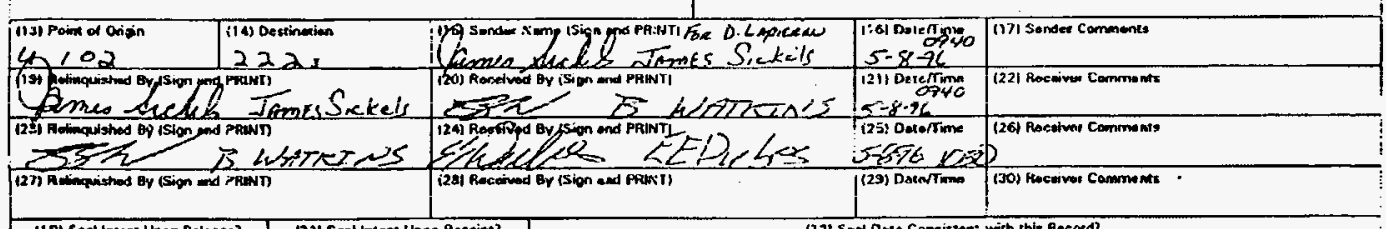
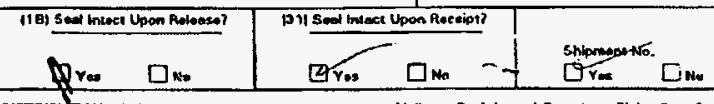

[32] Sell Oows Consistan: wich this Roeedd? 
(1) Shipment Number Doaci-08-TF

(4) rank $11-102 \quad$ (2) Sariple Number $960-189$

(4) Tank $14-102$. (5) Riser $q^{\text {(2) Sariple Number }} 96-189$

CHAIN-OF-CUSTODY RECORD FOR CORE SAMPLING

\begin{tabular}{|c|c|}
\hline $\begin{array}{l}\text { Radiotion Survay Date: } \\
\text { Over Top Dosa Rate }\end{array}$ & $\begin{array}{l}\text { (9) FIELD } \\
<.5\end{array}$ \\
\hline Side Dose Rate & 30 \\
\hline Bottom Doso Aaro & 7 \\
\hline \multirow[t]{3}{*}{ Smastablo Contamination } & $<20$ \\
\hline & $<1000$ \\
\hline & $\mathrm{SECl}_{\text {(signaturo) }}$ \\
\hline
\end{tabular}

(6) Segmanc

89

(3) supervisor M.C Jo nes

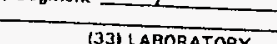
nicare 144

in INFOAMA TION (lnctide slacement of laboratory lests to be performed.

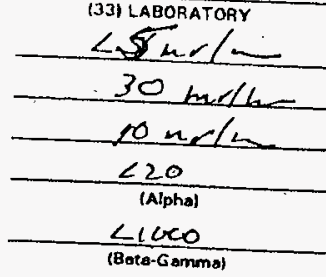

RCT. C. Cogece

(Ssionature)

in

(12) Field Cammon:s

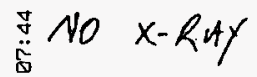

(0) 13) Point of Origin

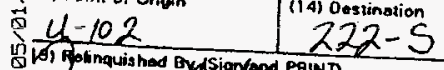

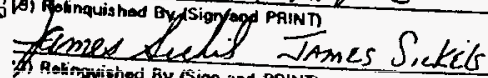

Whelingrisined EY ision and PRINT

(PT)

(1)

(10) Shipment Oescription
A. Work Package Nhmbor
B. Cosk Seal Number
C. Sampler Serial Number
D. Date and Time Sampter Unsastod
E. Expocted Liquid Coment
F. Expocted Solid Content
G. Dose Rato Through Drill String
H. Expocted Sample Langth

(8) cosk Sorist Number 02008

$-625-96-00059$ 10435

$94-3425$

$f-26-96 / 18: 15$

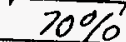

$30 \%$

$1.5 \mathrm{R} / \mathrm{HR}$ 171 (1) Relinguished or isign and PRINT)

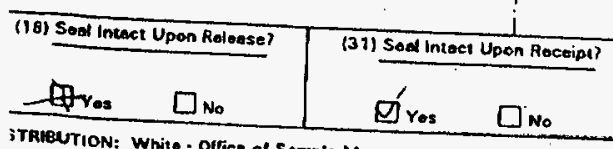

ITRLEUTION: White - Ofries of SAmmio Manas

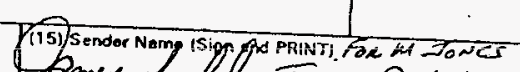

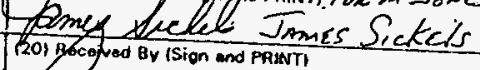
(24) gacoived ov ision and PAINT (28) Rocerned Armen L. beitaar
Shigmont No. Q
(34) Laboratory Comments

\section{ก๊y \\ $\therefore 5 \%$}

(16) Date/Time (17) Sonder Comments Sheyfe ost 12\}) Date/Timo [3/se ors 122) Rocaivar Comments (25) Dato/Time (26) Rocoiver Commonts fovise bs 45 129) Deto/Time (30) Receiver Comments 


\section{CHAIN-OF-CUSTODY RECOAD FOR CORE SAMPLING}

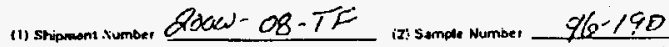

(4) Tort $-\mu-102$. 15 iniser 9

(3) suremisor Ma Jones

(n) $\mathrm{com}-144$

as Cas Sorid Number $c 1053$

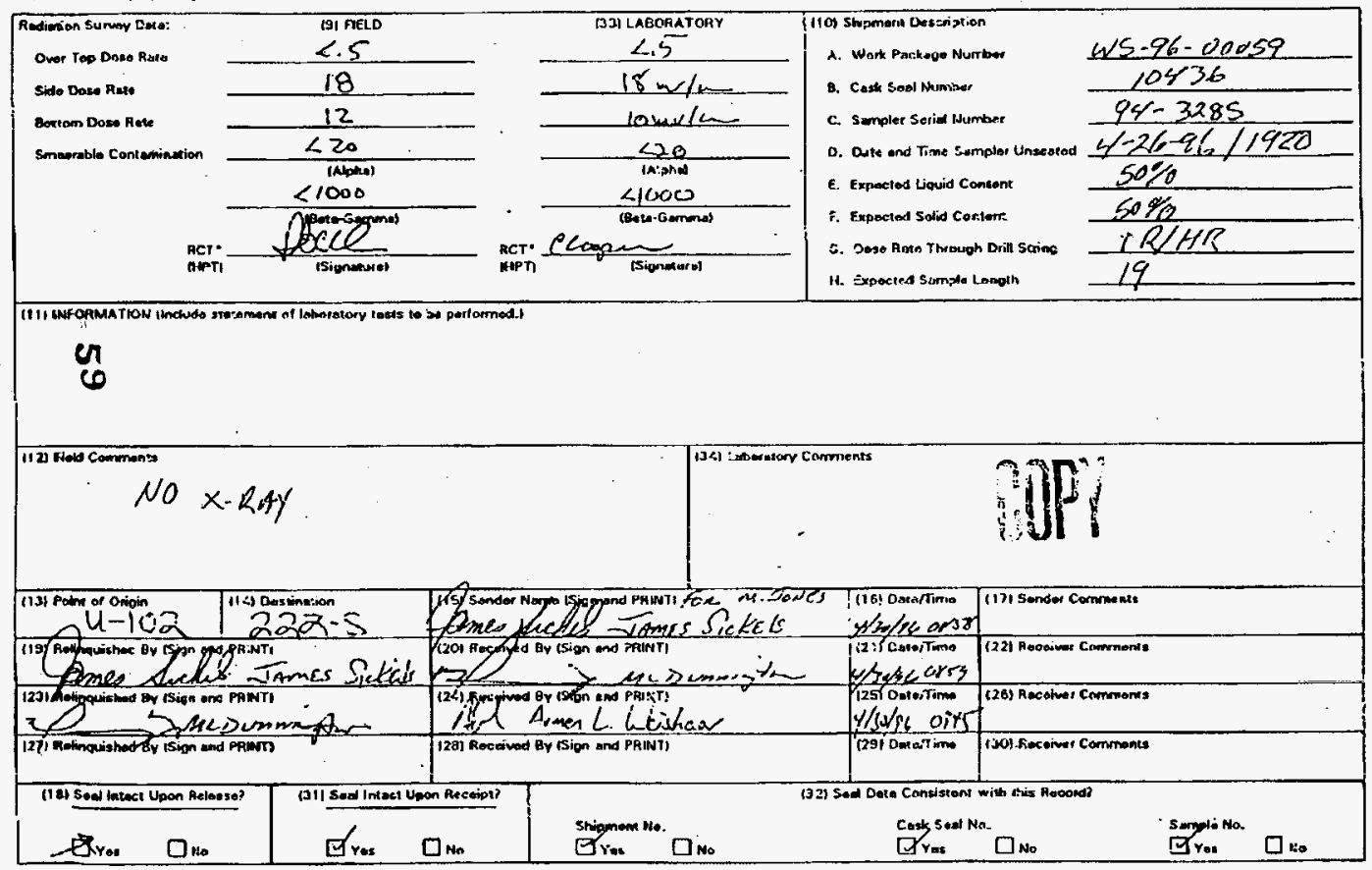

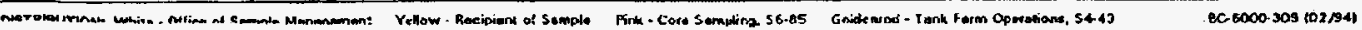


i1 Shimem Amme DOOW.OS-T\% CHAIN.OF-CUSTODY RECORD FOR CORE SAMPLING

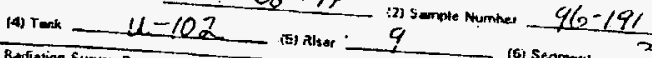

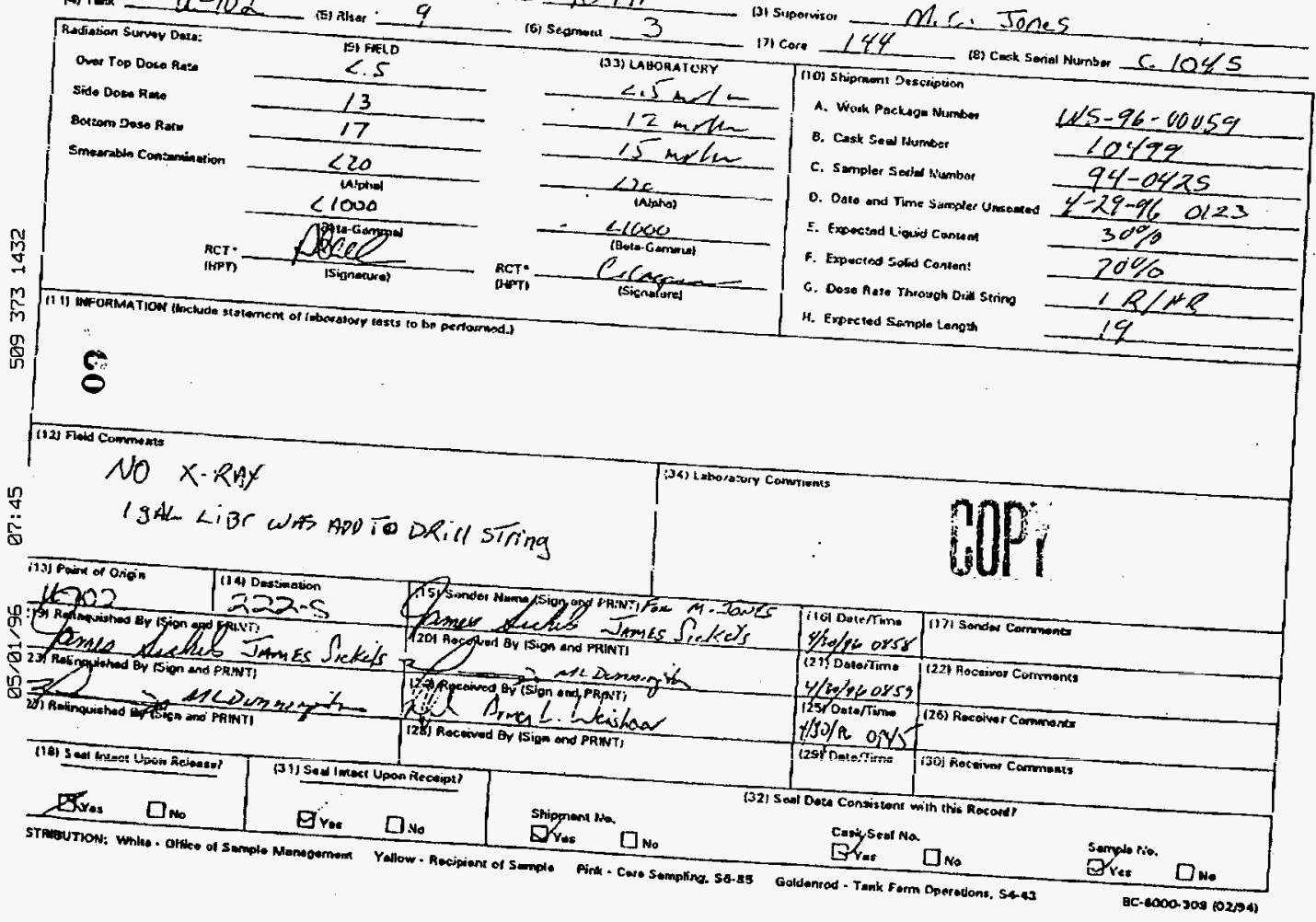




\section{CHAIN-OF-CUSTODY RECORD FOR CORE SAMPLING}

11 Shipment Number OPOOW-08-TF i) Tank $u-102$ (2) sample Number $-96-192$ (5) Riser +9

13i Supervisor Mici Jines

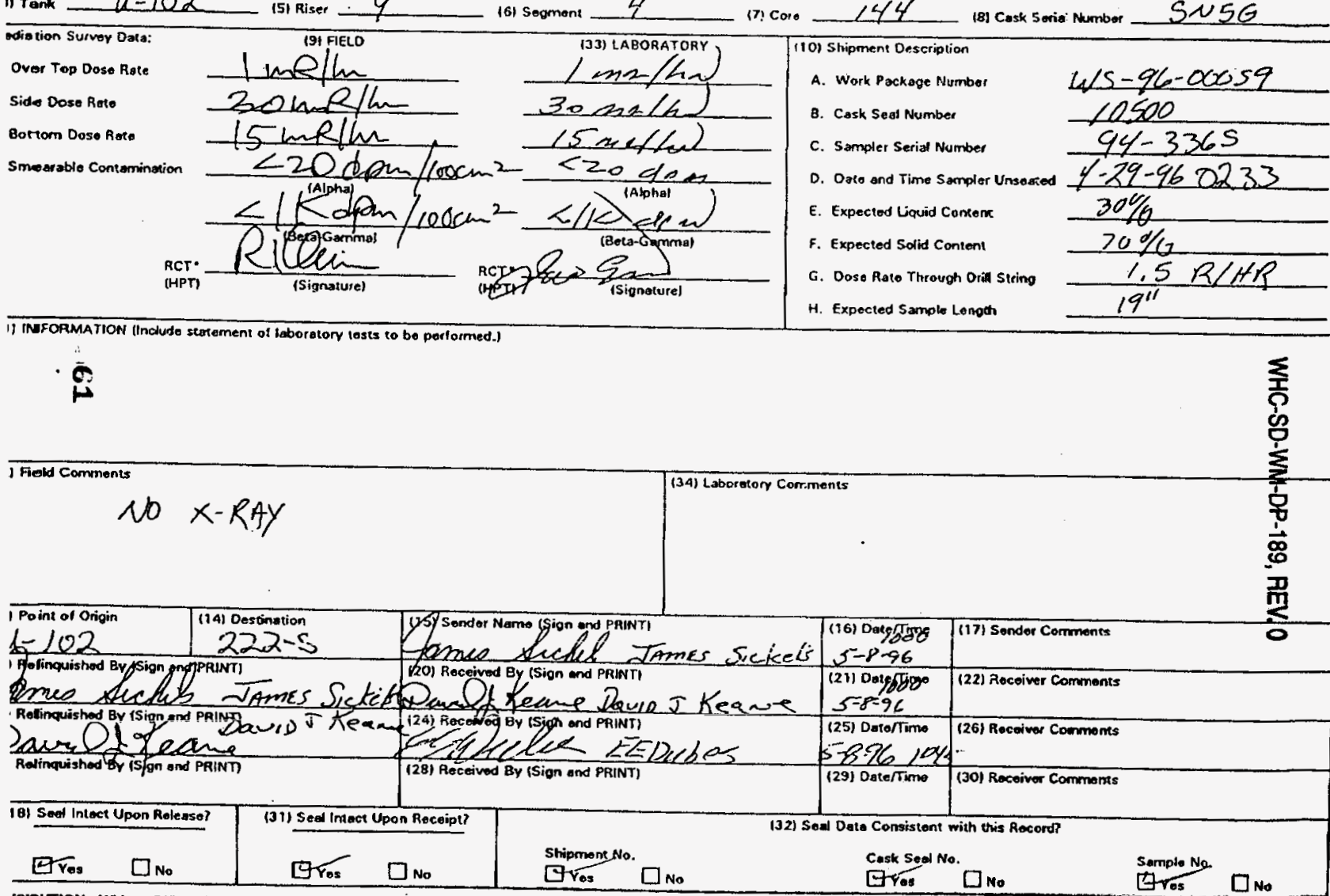




\section{CHAIN-OF-CUSTODY RECORD FOA CORE SAMPLING}

inrnent Number $200 \mathrm{~W}-08-T F \quad$ (2) Sample Number $96-193$ $4-102$ 151 Risee -9
161 segment 5
Oamen 2 Diann III (3) coro 144
(8) Cask Serial Number -58

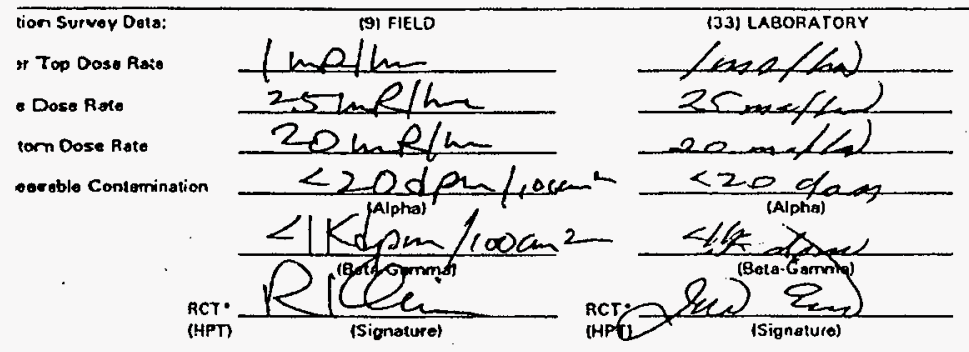

101 Shipment Oescription

\begin{tabular}{|c|c|}
\hline A. Work Package Number & $\simeq 5-76-0005910$ \\
\hline B. Cosk Seal Number & 1540 \\
\hline C. Sampler Serial Nuniber & $95-1605$ \\
\hline D. Dale and Time Sampler Unsoatod & $4 / 29 / 96 \quad 2205$ \\
\hline E. Expected Liquid Content & 107 \\
\hline F. Expected Solid Contem & Popo \\
\hline G. Dose Rate Through Orin String & $1.6 R / 4 R$ \\
\hline 4. Expected Sample Lenoth & $19^{4}$ \\
\hline
\end{tabular}

A. Work Package Numbe

$15-96-00059 / 0$ 1540

$95-1605$

C. Sampler Serial Nuniber

$4 / 29 / 96 \quad 220.5$

NF ORMA TiON IInchde stotemant of laborstory lests to be perlormed.)

Q

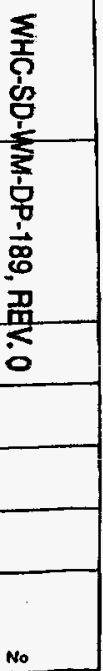

Sermpleftor. Qres $\square$ no

iedd commonts Broke apart between the Quadra- (34) Laboratory Commonis

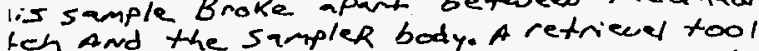

ich is a amadrakateh with two spring kadal

Is wene uret to retriece the sample. $x-1$ is

ill affached to the sampler Body.

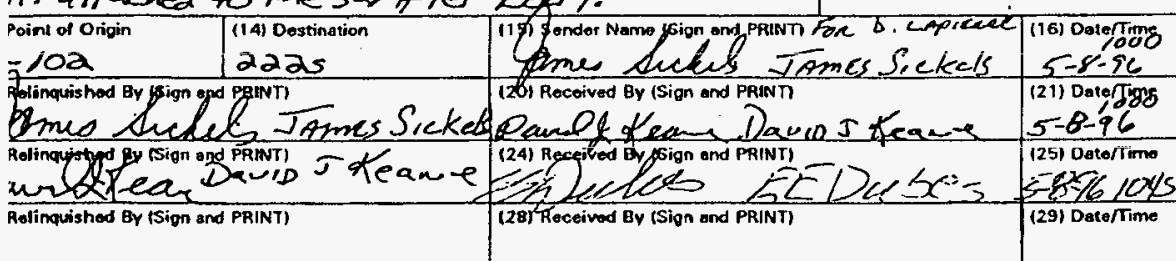

(17) Sonder Cormonts

(22) Recoiver Commonts

(26) F̄oceiver Cormmones

8) Sosl Intact Upon Reloase?

(31) Seed Imact Upon Receip\{?

Shipment No.

(32) Seat Dete Consistent with this Rocord?

Qres $\square$ No

Wo

$\checkmark$ Yos $\square$ No

Cosk Sealdo:

Gres $\square$ No

(9C-6000-309 (02/94) 
CHAIN-OF-CUSTODY RECORD FOR CORE SAMPLING

ihiponent Number $0200 \omega-08-T F$

121 somple Number $-96-194$

(3) Supervisor $M C_{1}$. Jones

(8) Cosk Seriel Number $c / 033$ ink $-11-102$ (5) Aiser .9

(G) Sogment 6

17) core 144

ation Surver Dato:

ver Top Dose Rate

de Dose Reto

$\frac{25 m+2 / 4}{16 m}$

133) LABORATORY

ntom Oosa Rato

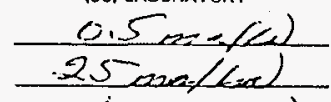

-. $\left\langle 0 a_{1}(\alpha)\right.$

neorablo Contaminstion

<20 dpur/conam ${ }^{2}$ $x=0$ open

RCT. $1 \mathrm{Kopm}^{\text {(Alphal } / 100 \mathrm{Cum}^{2}}$

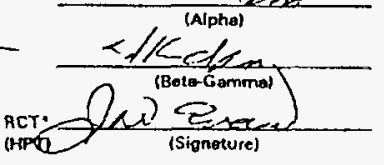

(10) Shipment Description

A. Work Package Nurntibat

B. Cask Seal Number

C. Sampler Seriar Number

w5-96-00059

10459

D. Dato ond Time Sampitat Unso ated

$95-167-5$

E. Expected Liquid Content

F. Expected Solid Content

G. Dose Rate Through Drill String

H. Expected Sample Length

$z-30-760310$

$30 \%$

$20 \%$

$1.7 \mathrm{R} / \mathrm{H} B$

INFORMATION (linclude statemem of leborosary cesta to be performed.)

क)

\section{Field Cormonts}

VO $X-R A Y$

SAMple wAS onvy pushed 13" Do To high. Down force.

\section{Poim of Origin}

$i-102$

(14) Descination

$222-5$

eli mquishod Bypsign gnc,paivT!

rearea secks =

JAP

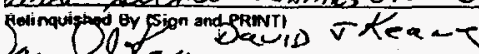

tann 2 teane

Retinquishod B. (Sign and PRINT)

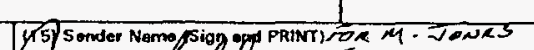

8) Soel Intoct Upon Releosel

91 Soel Intoct Upon Releaser?

Dros DNo

Disand Keanes.

(24) Aeceiyugd 8. 1Sion and PRINT)

(28) Poceived By (Sign ond PRINT)

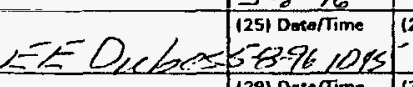

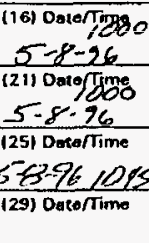

(17) Sender Comments

(22) Receiver Comments

(26) Recoival Comments

(30) Roceiver Cornments

(32) Seet Dato Consistent with this Record?

Shipriong Ato.

Cosk SoghNo.

ares $\square$ No

Semple Nor

Qros $\square$ No

No

Gros

[No 


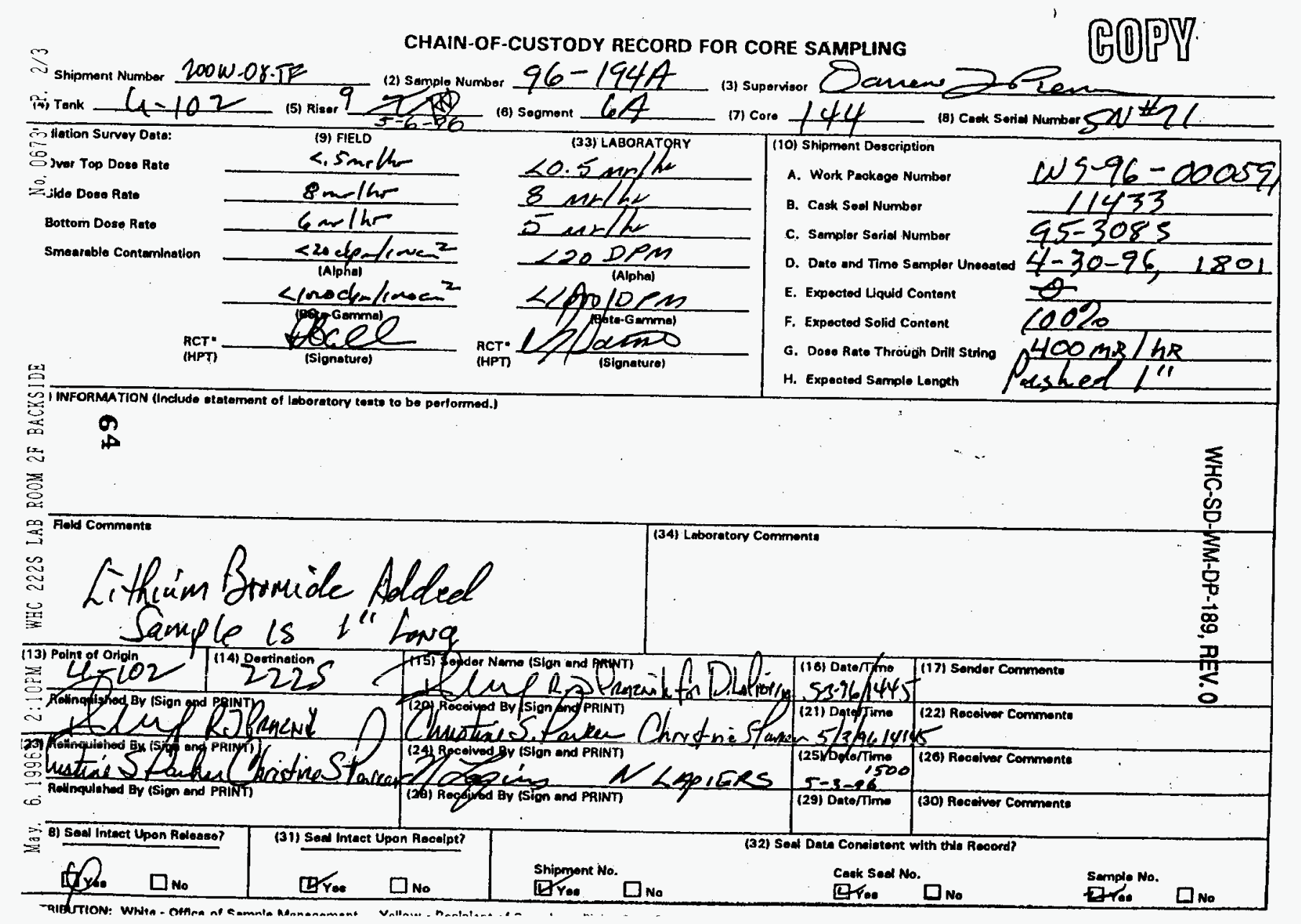




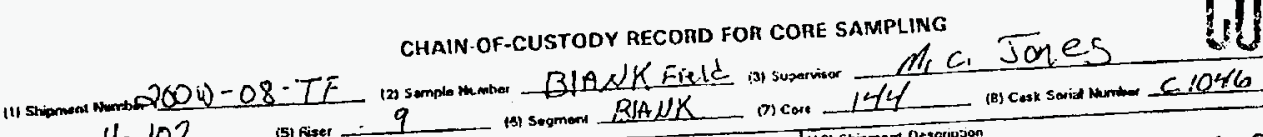

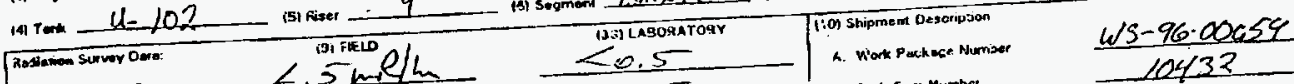

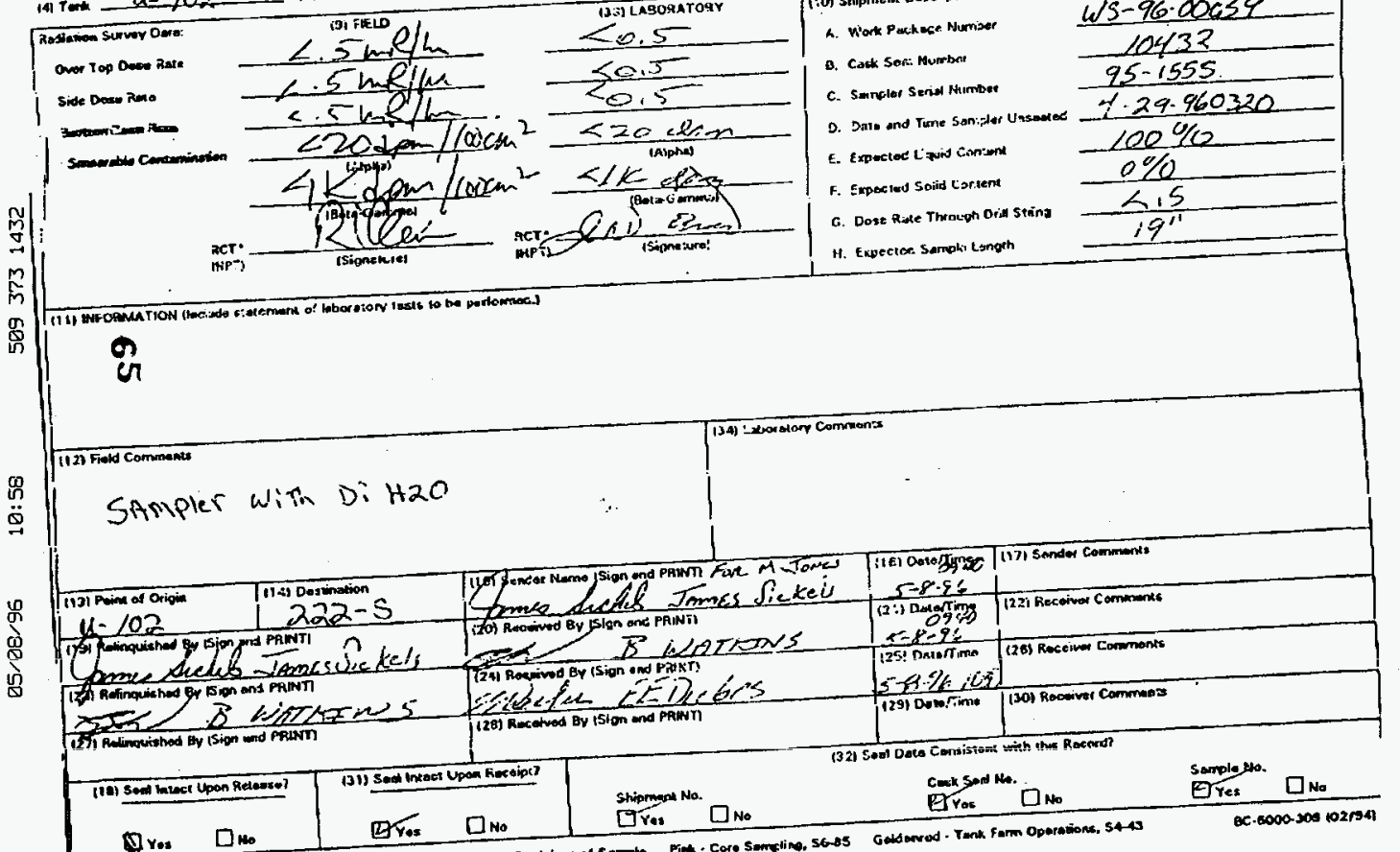

Q Pros

Deros DNo

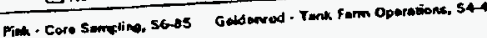




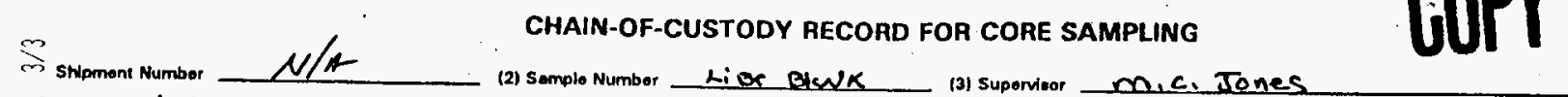
ai, Tank $-U=102$ (5) Rher 9 (6) Sogment Blank (7) core 144

(B) Cenok Sortel Number $N / A$

\begin{tabular}{|c|c|}
\hline 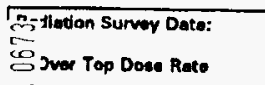 & $\begin{array}{l}\text { (9) FIELD } \\
<.5\end{array}$ \\
\hline 注 & c. 5 \\
\hline Bortram Dose Hete & $<.5$ \\
\hline Smoorsoble Contenmination & $<20$ \\
\hline & $\begin{array}{c}\text { (Alpha) } \\
</ 010\end{array}$ \\
\hline & 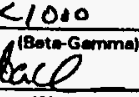 \\
\hline
\end{tabular}

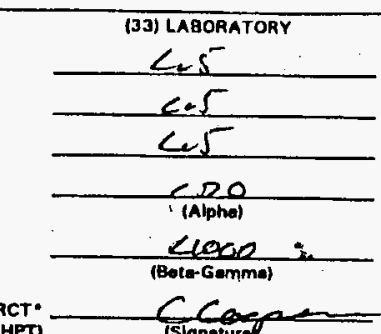

\begin{tabular}{|c|c|}
\hline \multicolumn{2}{|l|}{ (10) Shipmont Description } \\
\hline A. Work Peckege Number & $w s-96-\infty 0059$ \\
\hline 8. Cank Sod Number & 10480 \\
\hline C. Sempler Sarien Numbar & $N / A$ \\
\hline D. Date and TIme Sampler Uneostrod & $4-30-96$ \\
\hline E. Expoeted Liquid Content & $100 \%$ \\
\hline F. Expociod Solid Content & $0 \%$ \\
\hline C. Dose Rate Through Drll String & $<.5 \mathrm{MR} / \mathrm{HR}$ \\
\hline H. Expected Sample Longth & $100 \mathrm{~mL}$ \\
\hline
\end{tabular}

in ORMA TION Inodude statemont of leboretory tasts to be performed.)

isionaturef

के

$\%$

a Fold Commente

舀

$\underset{\Xi}{\approx}$ iBr Blank for Tauk $u-102$

骎

(13) Polne of Ondein

zu 102

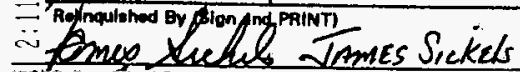

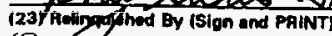

8

$$
\text { क }
$$
cis

18) Seed Inteot Upon Rolecese? $\geq$

Fro

$\square$ No

(2)

exmang ite

\section{Ris} ander Nongo (Sjoyand PRINT TOR M.Jomes

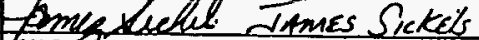

(16) Dete/Timo (17) Sender Commonte (20) Botoglued Oy (Stan and PRINT) 
WHC-SD-WM-DP-189, REV. 0

INORGANIC ANALYSES

$=167$ 
WHC-SD-WH-DP-189, REV. 0

THIS PAGE WAS INTENTIONALLY LEFT BLANK

$: 68$ 
worklistrpt Version $2.105 / 15 / 95$

WHC-SD-WM-DP-189, REV. 0

Page: 1

05/28/96 12:33

LABCORE Data Entry Template for Worklist\#

9257

Analyst: COPM Instrument: DSC0 1 Book \#12 NHP

Method: LA-514-113 Rev/Mod C-I

Worklist Comment: U-102 DSC RUN UNDER N2. RCJ

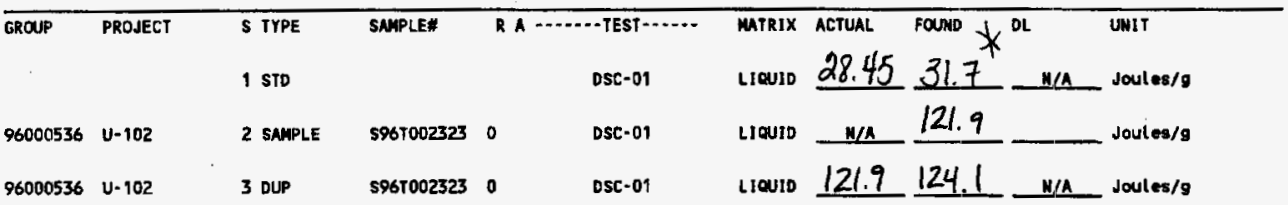

Final page for worklist \# $\quad 9257$

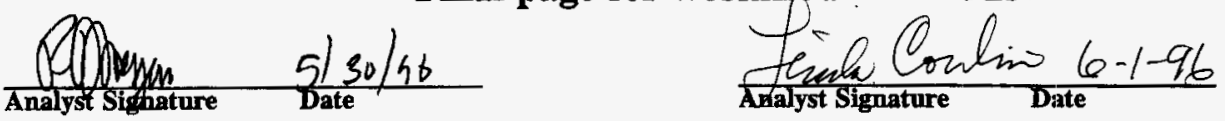

$$
\text { Validated by Hanasto 6/2/96 }
$$

Data Entry Comments: Sample results are the sum of two exotherms.

Units shown for $Q C$ (SPK \& STD) may not reflect the actual units. $D L=$ Detection Limit, $S=$ Worklist Slot Number, $R=$ Replicate Number, $A=$ Aliquot Code.

$-69$ 
SIGNATURE ABOVE REPRESENTS CHEMICAL TECHNOLOGIST/CHEMIST THAT

COMPLETED/VERIFIED THE CALIBRATION/ANALYSIS ON PAGES 70 TO 72 .

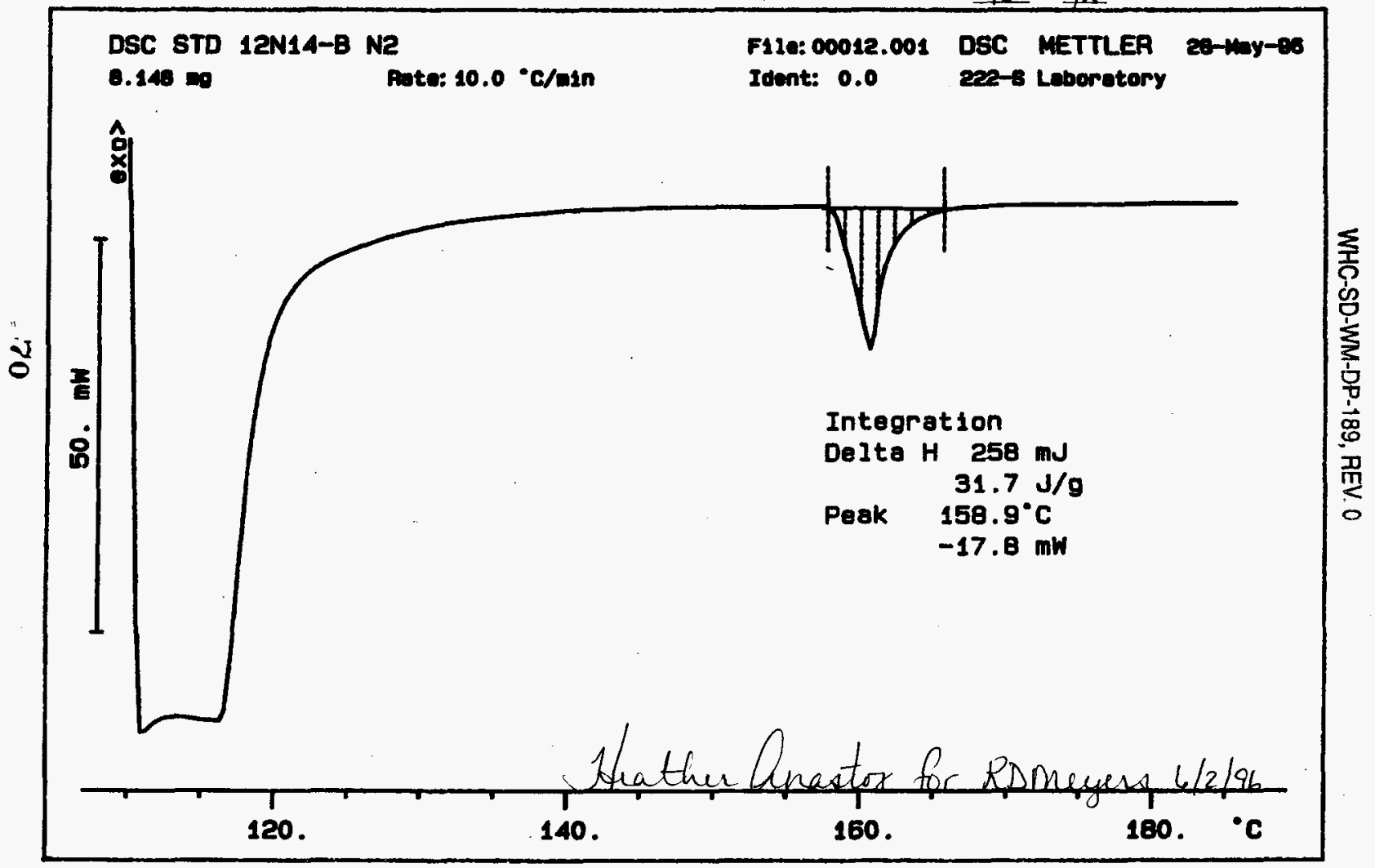


WHC-SD-WM-DP-189, REV O

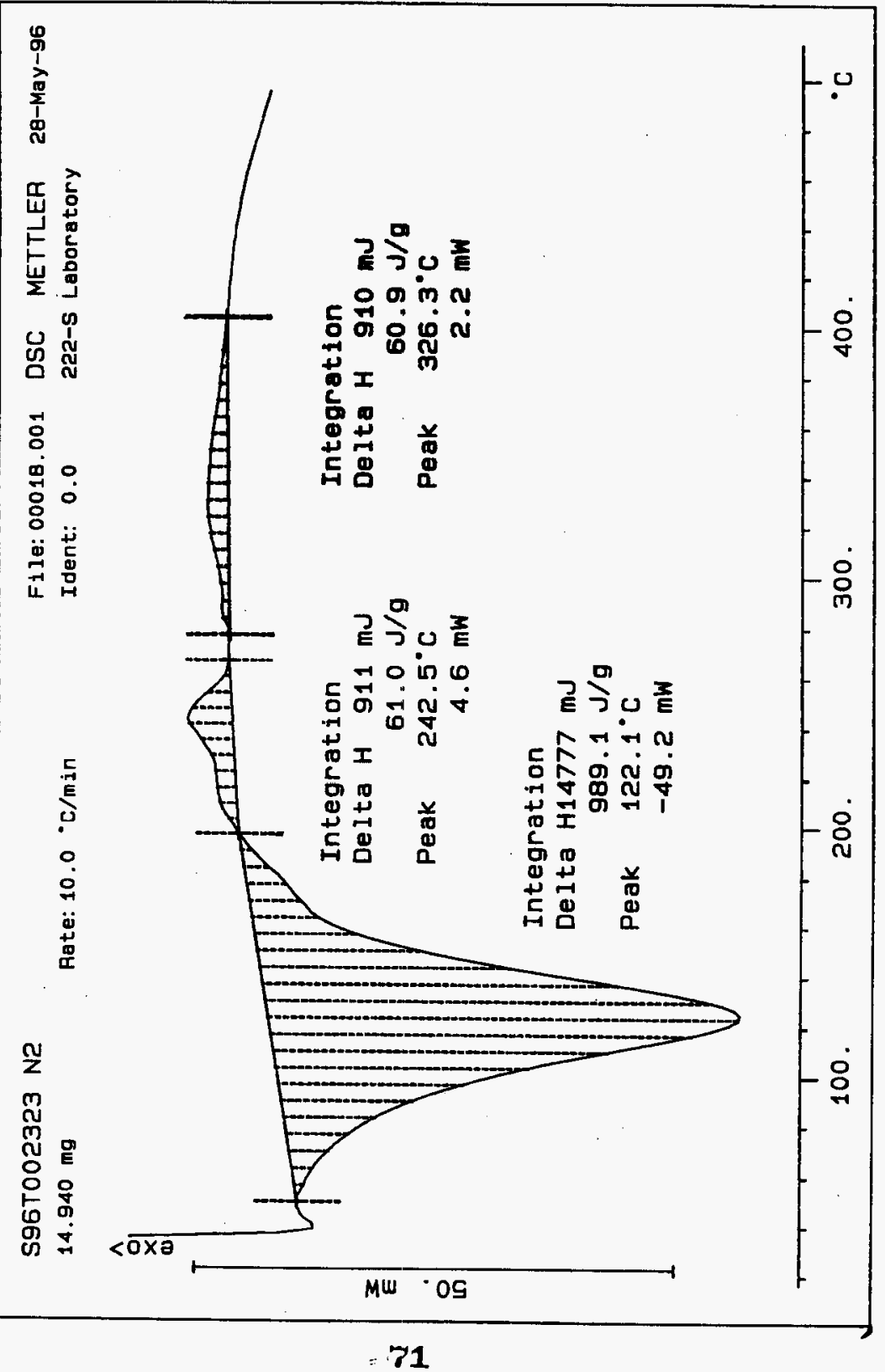




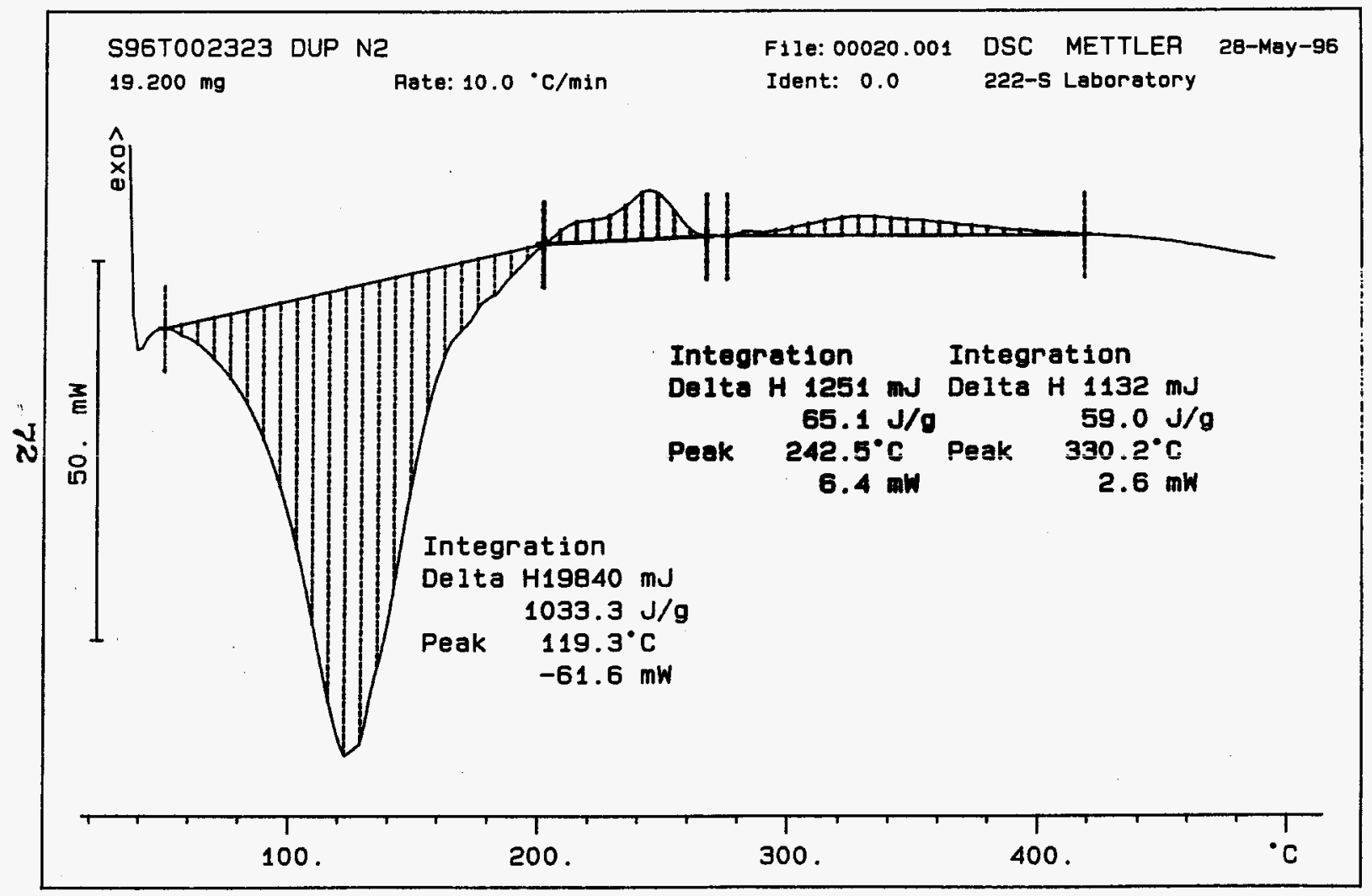




Analyst: $\quad$ RDM Instrument: DSC0 3 Book \# 12 N14B

Method: LA-514-114 Rev/Mod C-1

Worklist Comment: U-102 DSC RUN UNDER N2. RCJ

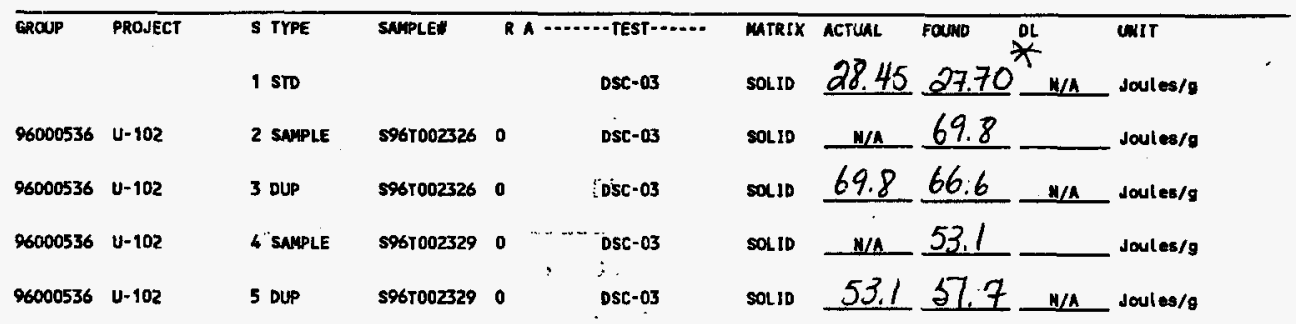

\section{Final page for worklist \# 9258}

See attached for signatures

Analyst Signature Date $6 / 13 / 96$
Validated by Hanaotn $6-19.96$

Row $6.17-96$

Analyst Signature

Date

Data Entry Comments:

Units shown for $Q C$ (SPK \& STD) may not reflect the actual units. $D L=$ Detection Limit, $S=$ Worklist Slot Number, $R=$ Replicate Number, $A=$ Aliquot Code. 
WHC-SD-WM-DP-189, REV. 0

worklistrpt Version $2.105 / 15 / 95$

$05 / 28 / 96 \quad 12: 35$

\section{LABCORE Data Entry Template for Worklist\#}

Analyst: RPON Instrument: DSCO Book \# $12 N 14 B$

Method: LA-514-113 Rev/Mod ( $)$

Worklist Comment: U-102 DṢC RUN UNDER N2. RCJ

\begin{tabular}{|c|c|c|c|c|c|c|c|c|c|c|}
\hline \multirow[t]{2}{*}{ croup } & \multirow[t]{2}{*}{ PROJECT } & S TYPE & SAMPLE" & \multicolumn{2}{|c|}{ R A $\cdots$ - . TEST $\cdots .}$. & \multirow{2}{*}{$\begin{array}{l}\text { MATRIX } \\
\text { SOLID }\end{array}$} & \multirow[t]{2}{*}{ ActuAl } & \multirow[t]{2}{*}{ FOUND } & \multirow[t]{2}{*}{ OL } & \multirow{2}{*}{$\begin{array}{l}\text { UNIT - } \\
\text { Joules/g }\end{array}$} \\
\hline & & 1 STb & & & DSC-01 & & & & & \\
\hline 96000536 & $v-102$ & 2 SAMPLE & $\$ 961002326$ & 0 & DSC-01 & $50 L 10$ & $\mathbf{N} / \mathbf{A}$ & & & Joules/g \\
\hline 96000536 & $U \cdot 102$ & 3 DUP & 5967002326 & 0 & DSE-01 & SOLID & & & $\mathbf{n} / \mathbf{A}$ & Joules/g \\
\hline 96000536 & $U-102$ & 4 SAMPLE & 5961002329 & 0 & DSC-01 & SOLIo & $\mathrm{N} / \mathrm{h}$ & & & Joules/9 \\
\hline 96000536 & U-102 & 5 DUP & $\$ 967002329$ & 0 & $D S C=01$ & SOLID & & & $\mathbf{N} / \mathbf{A}$ & Joules/o \\
\hline
\end{tabular}

\section{Final page for worklist \# $\quad 9258$}

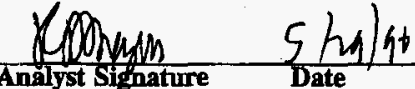

\section{什}

Analyst Signature Date

\section{Analyst Signature Date}

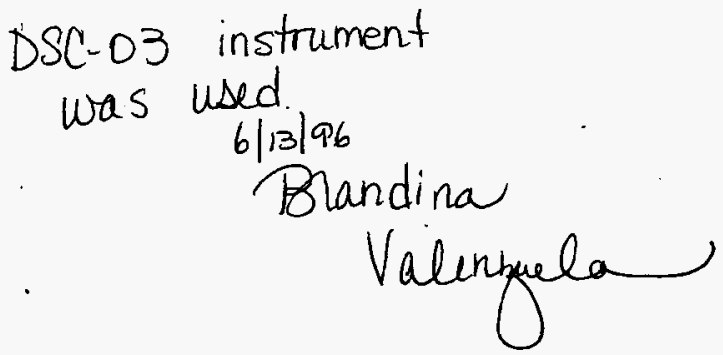

Date Entry Comments:

Units shown for QC (SPK \& STD) may not reflect the actual units. $D L=$ Detection Limit, $S=$ Worklist Slot Number, $R=$ Replicate Number, $A=$ Aliquot Code. 


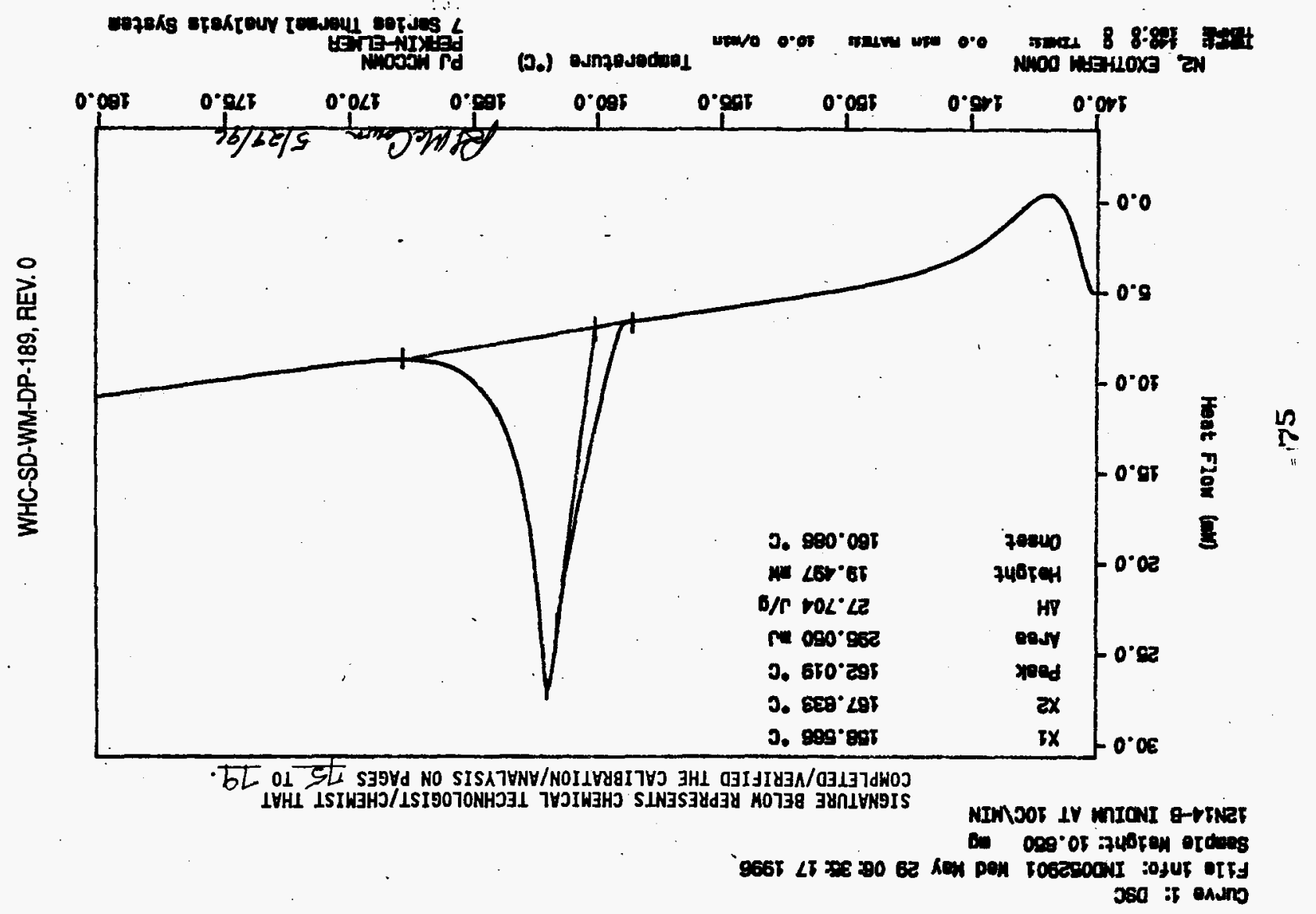


Curve 1: DSC

File Info: SAM05e905 hed May 29 18:5er 461996

Somplo Meight: 13.200

596T002326

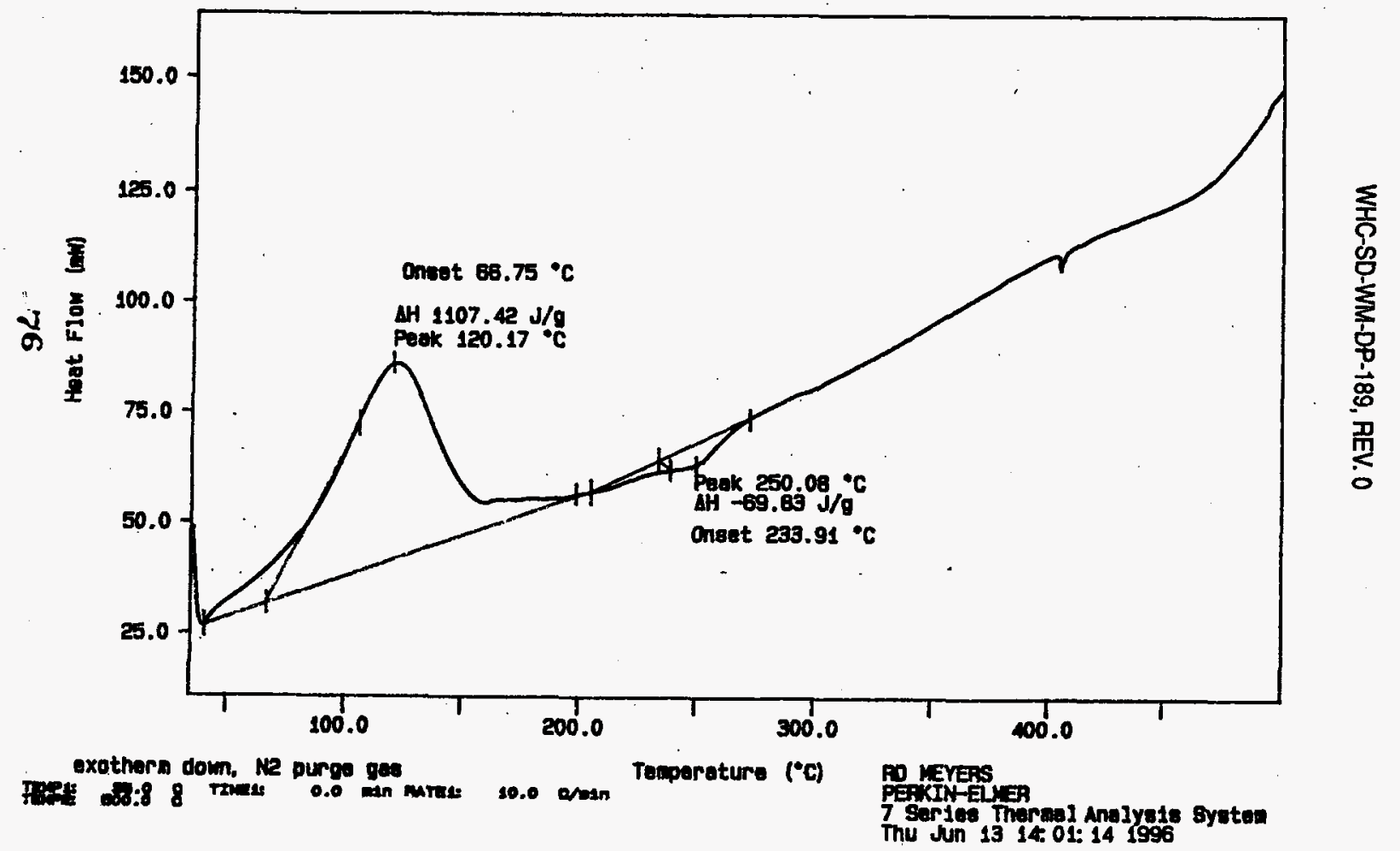


Curve 1: DSC

F118 info: SAMNSe906 Wod May ag 20: 30: 161996

Semple Woight: 17.370

sgstoozazanup

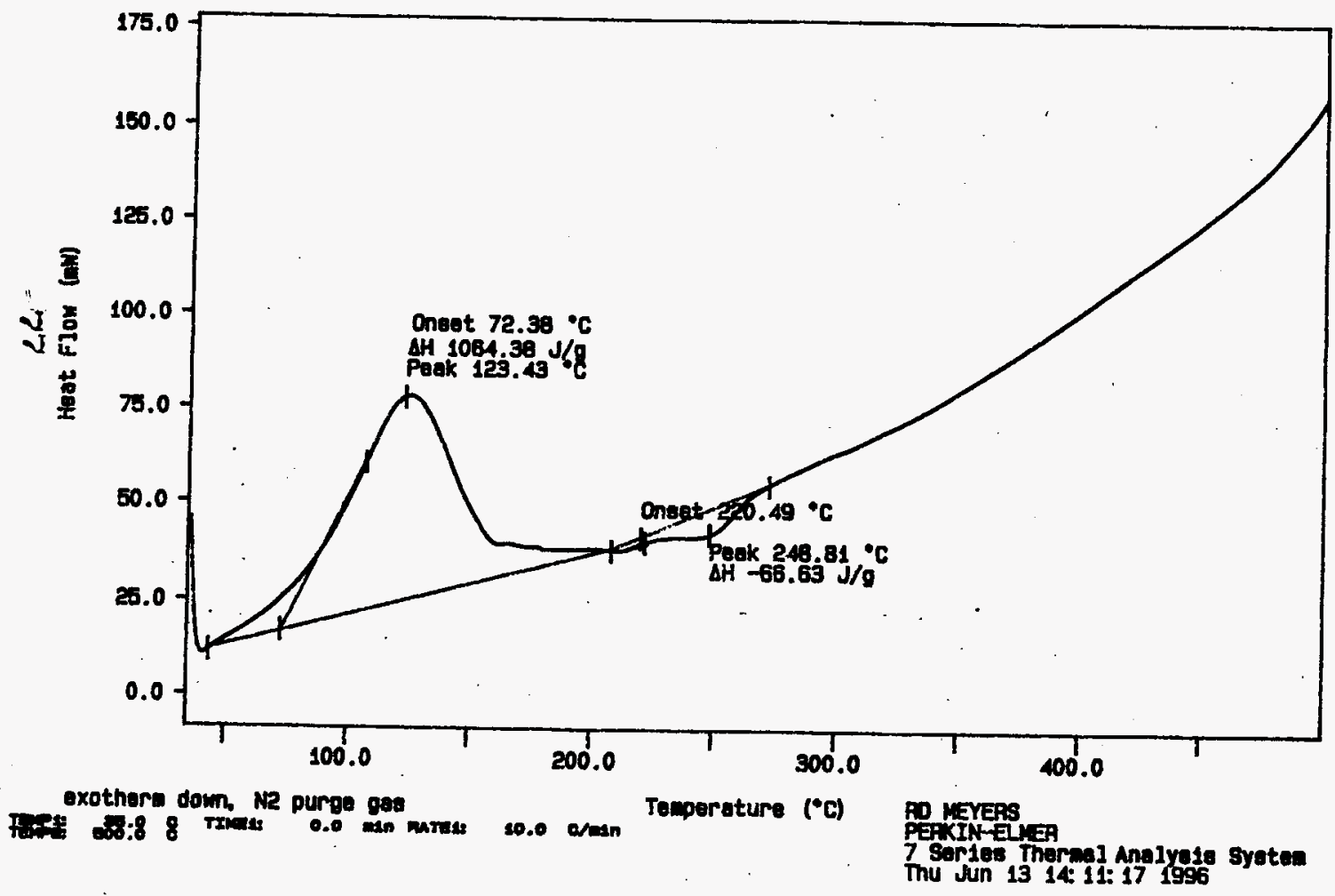


WHC-SD-WM-DP-189, REV. 0

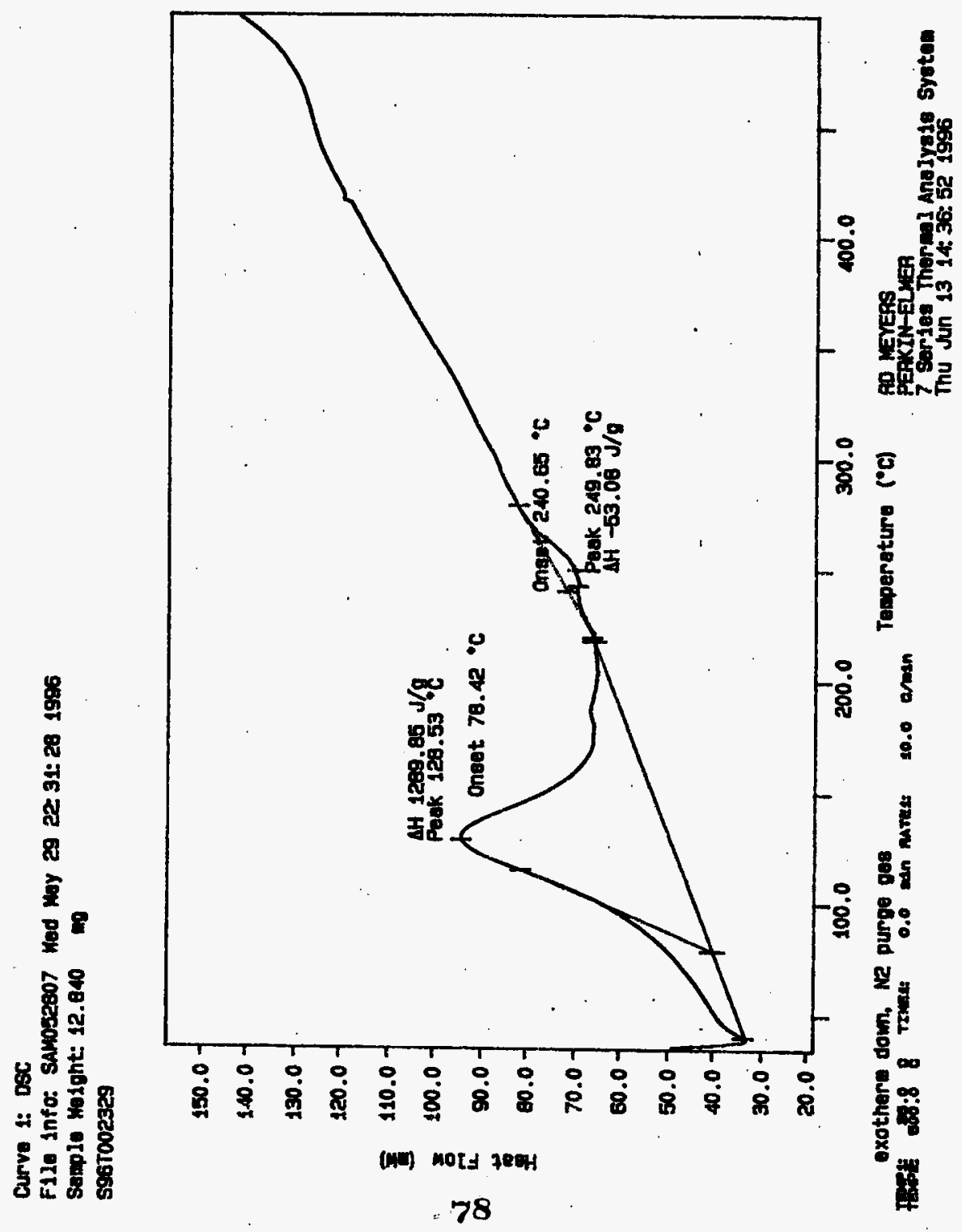


WHC-SD-WM-DP-189, REV. 0

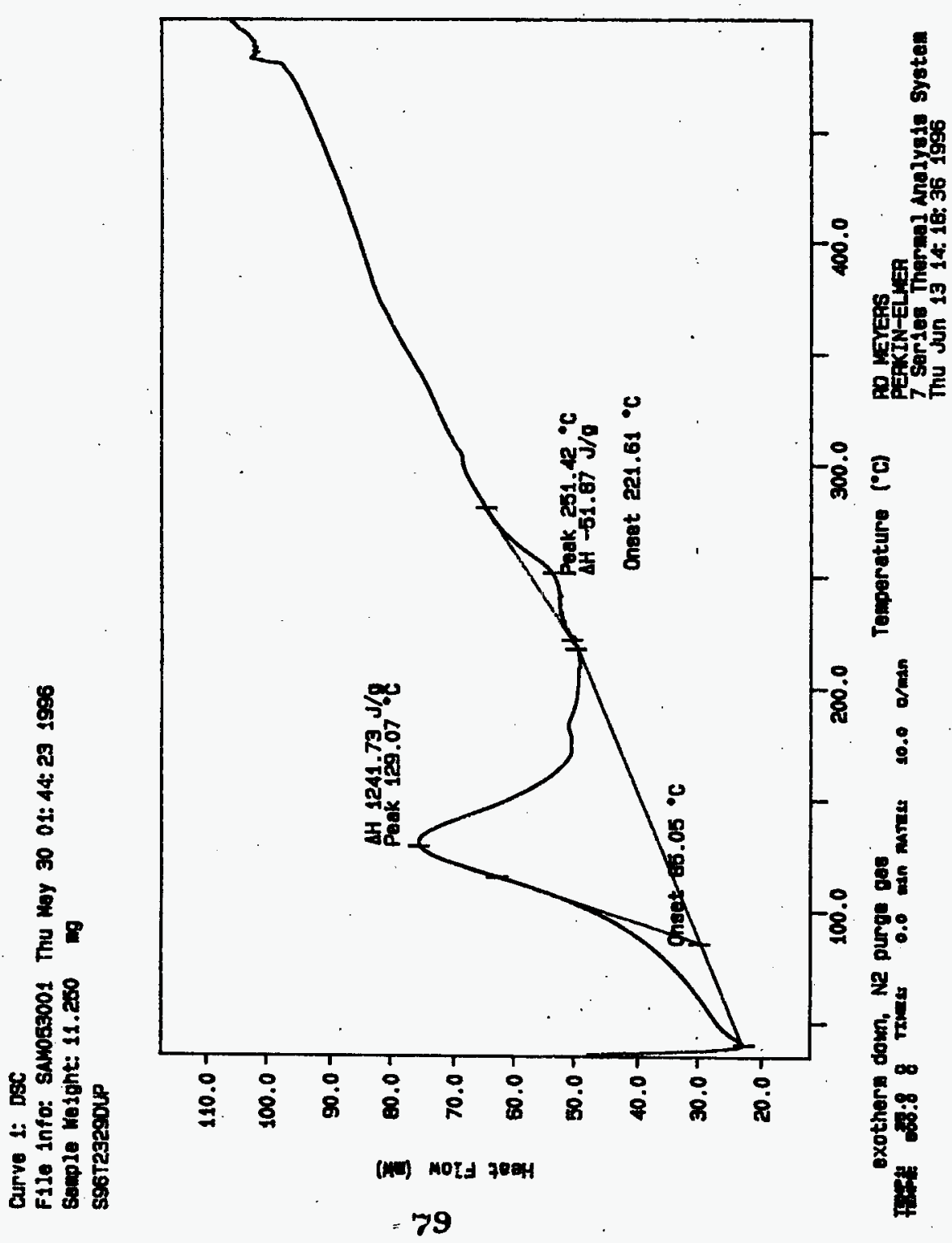


worklistrpt Version 2.1 05/15/95 05/28/96 12:36

LABCORE Data Entry Template for Worklist\#
Page: I

9259

$\begin{array}{ll}\text { Analyst: } \operatorname{ROM} & \text { Instrument: DSCO } 1 \text { Book \#12N14B } \\ \text { Method: LA -514-113 Rev/Mod } \mathrm{C}-1 & \end{array}$

Worklist Comment: U-102 DSC RUN UNDER N2. RCJ

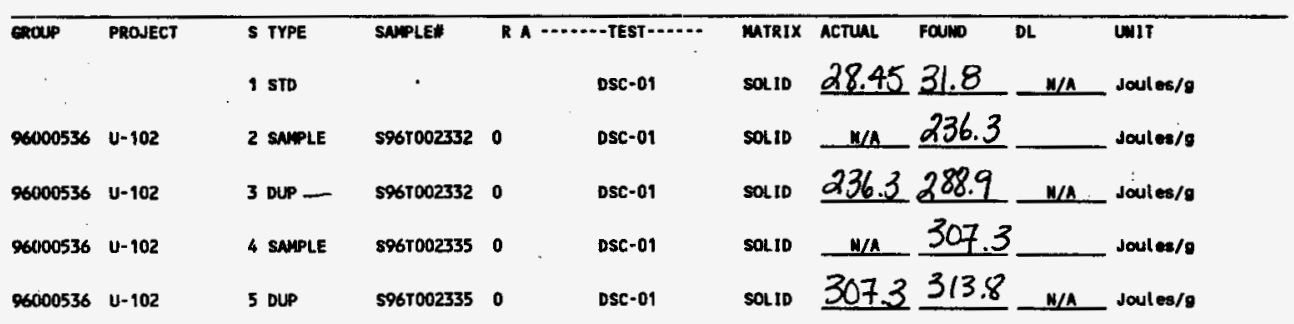

Final page for worklist \#

9259
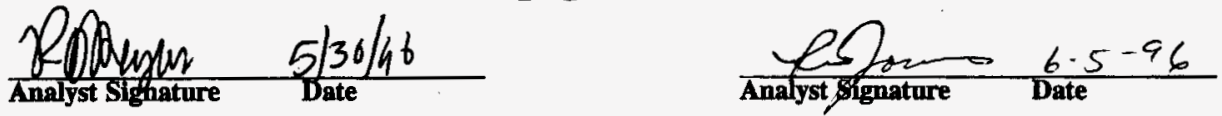

Validated by

Hanaosto 6.6.96

S967002332 results are the sum of 3 exotherms. The exotherm at approximately $450^{\circ} \mathrm{C}$ is probably due to a decomposition of a compound which was relatively pure.

Date Entry connersis 3967002335 results are the sum of 3 exotherms. The exotherm at approximately $450^{\circ} \mathrm{C}$ is probably due to a decomposition of a relatively pure component.

Units shown for $Q C$ (SPK \& STD) may not reflect the actual units. $D L=$ Detection Limit, $S=$ Worklist Slot Number, $R=$ Replicate Number, $A=$ Aliquot Code.

80

田

$\sqrt{2}$ 


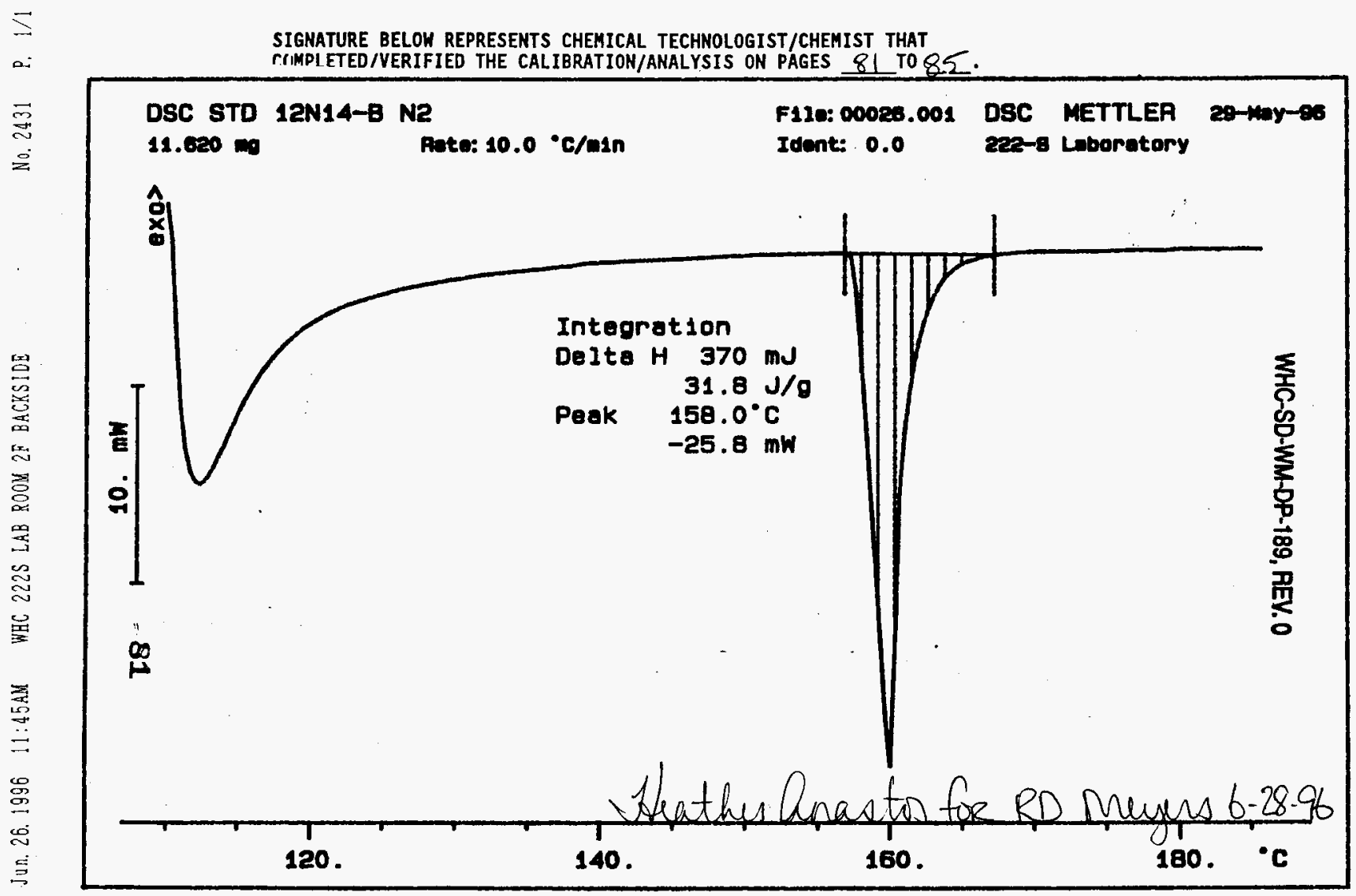




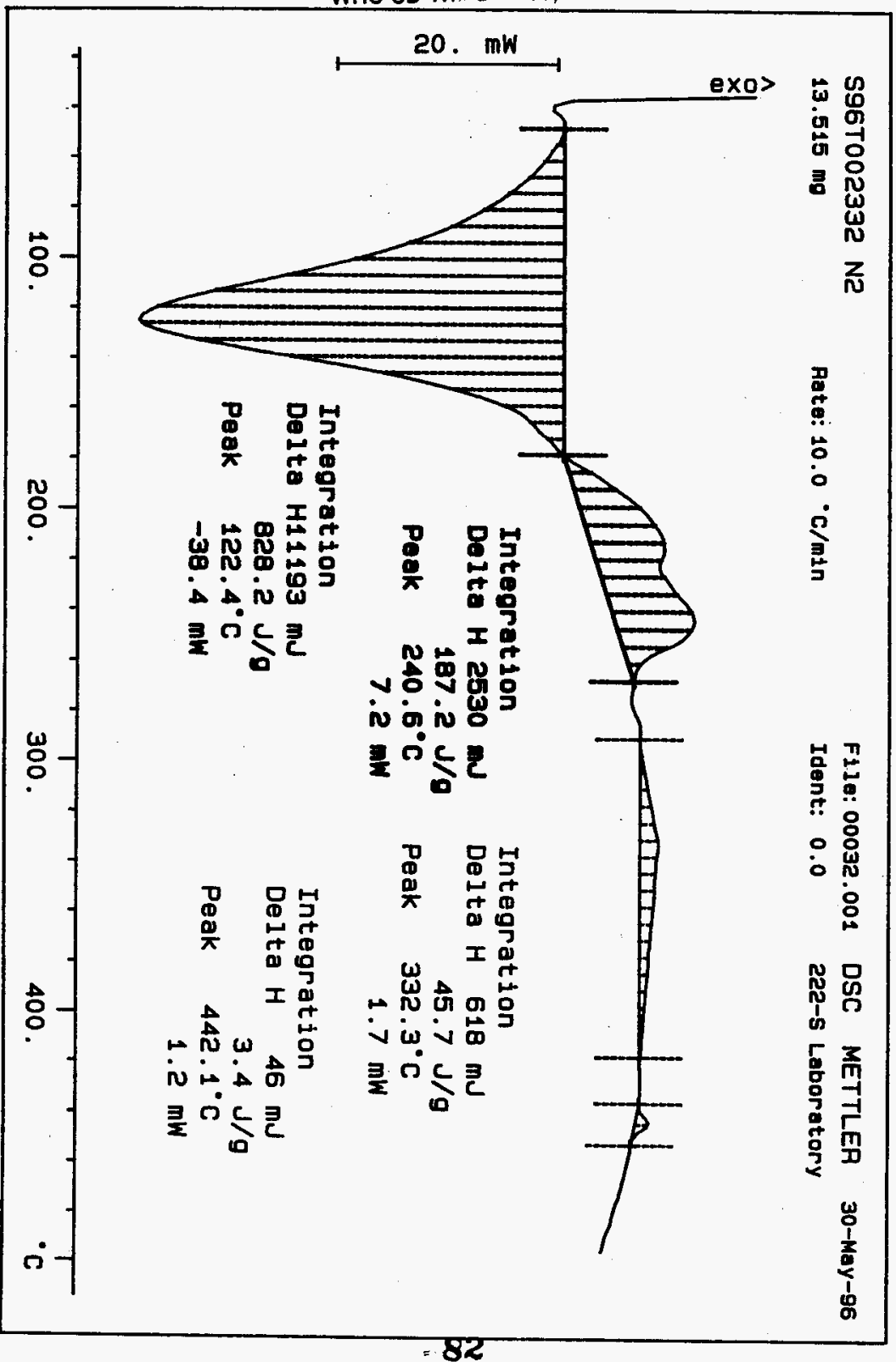




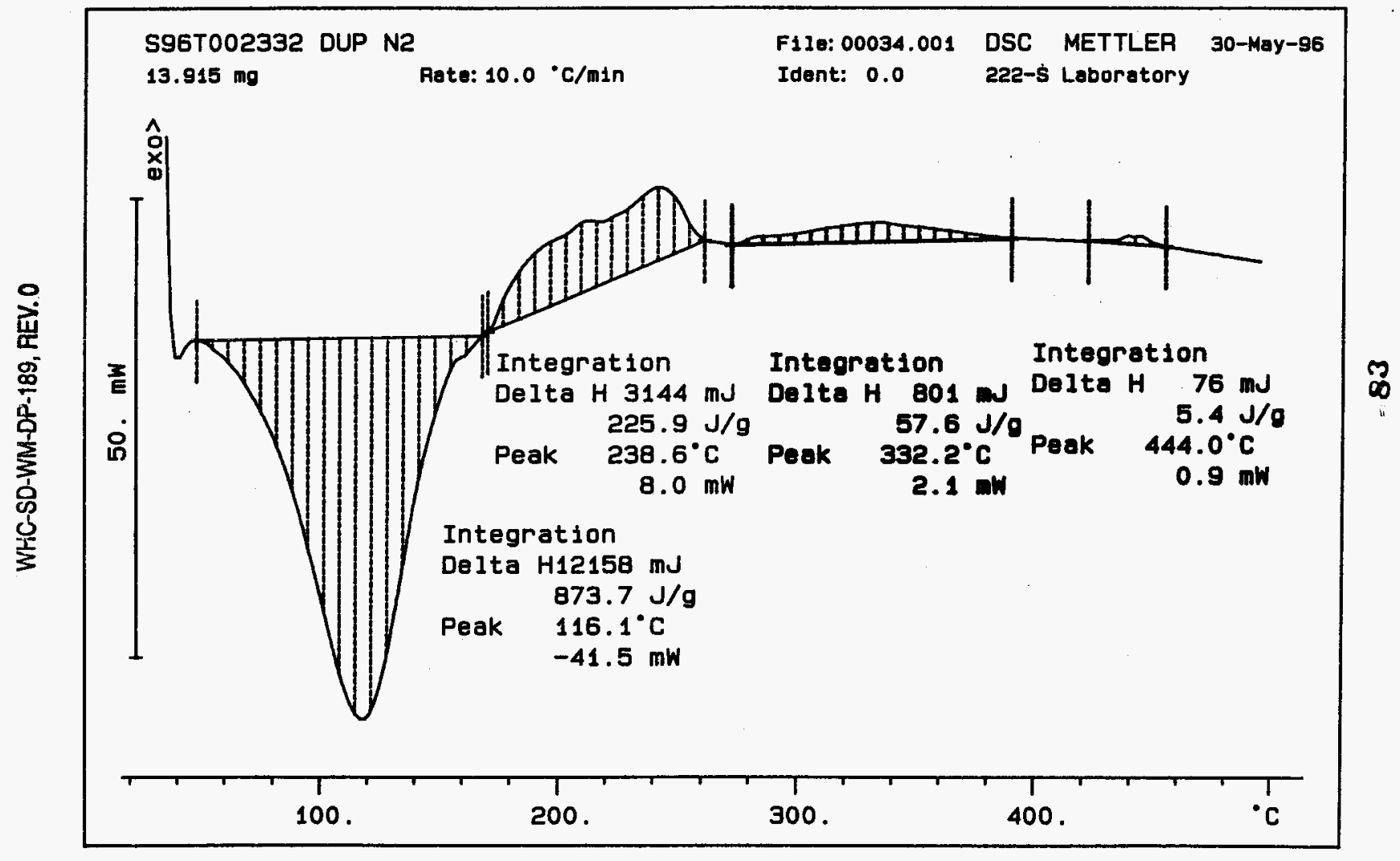




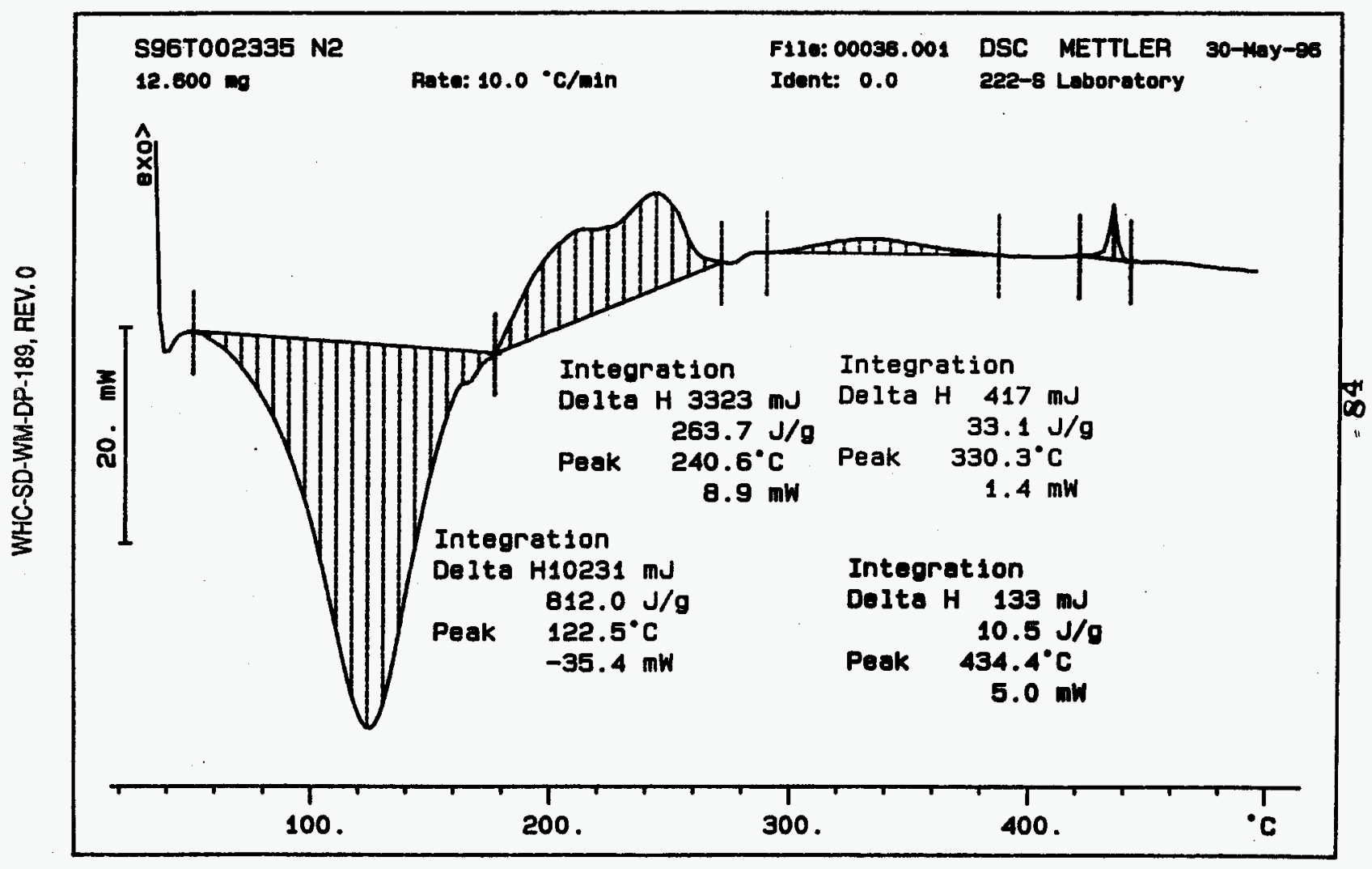




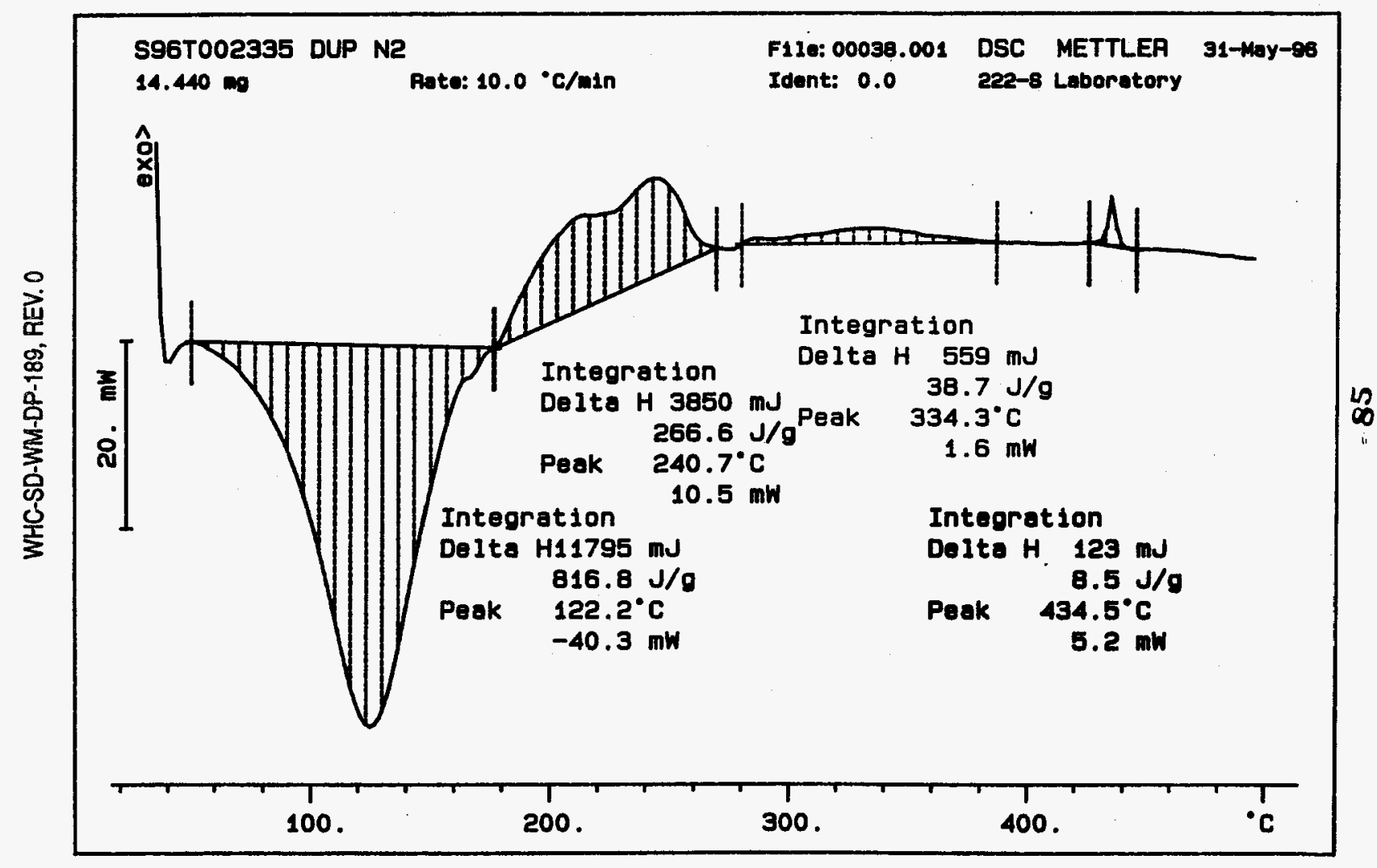


worklistrpt Version $2.105 / 15 / 95$

WHC-SD-WM-DP-189, REV. 0

$05 / 78 / 9612.40$

LABCORE Data Entry Template for Worklist\#

Page: 1

9260

Analyst: $\quad \mathrm{KRm}$ Instrument: $\mathrm{DSC} 11$ Book \# 12 N14B

Method: LA-514-113 Rev/Mod CC I

Worklist Comment: U-102 DSC RUN UNDER N2. RCJ

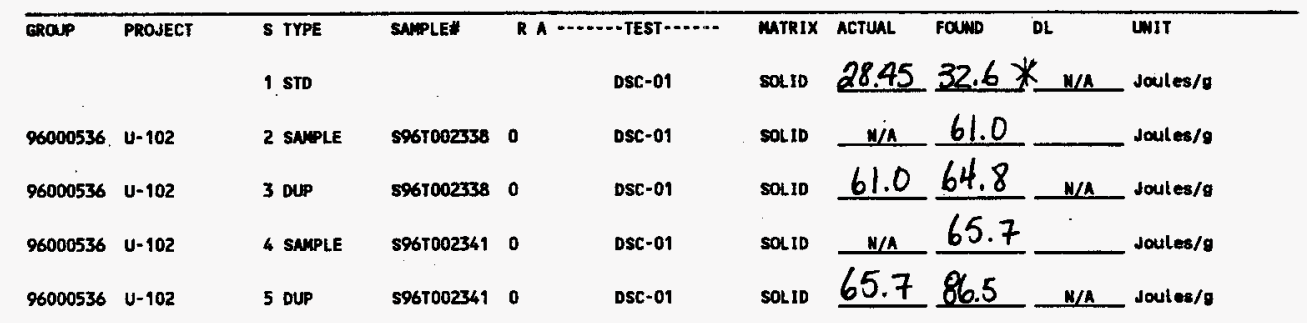

Final page for worklist \# 9260

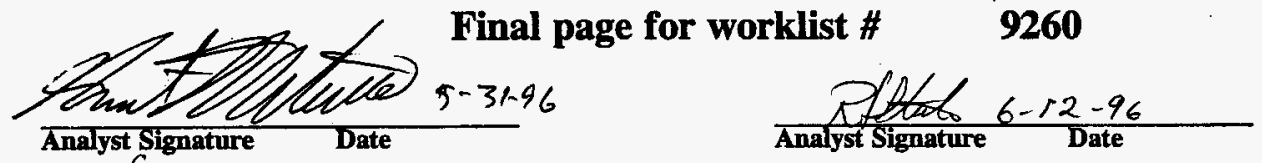

Verified Validated by

Banding valentoleda 6114196

Date Entry Cones: The chem tech integrating sample 5967002341 was a little over zealous in his integration. She results crossed out are excess results.

Units shown for QC (SPK \& STD) may not reflect the actual wits. $D L=$ Detection Limit, $S=$ Worklist Slot Number, $R=$ Replicate Number, $A=$ Aliquot Code.

$=86$ 
SIGNATURE BELOH REPRESENTS CHEMICAL TECHNOLOGIST/CHEMIST THAT

COMPLETED/VERIFIED THE CALIBRATION/ANALYSIS ON PAGES 87 TO 91.

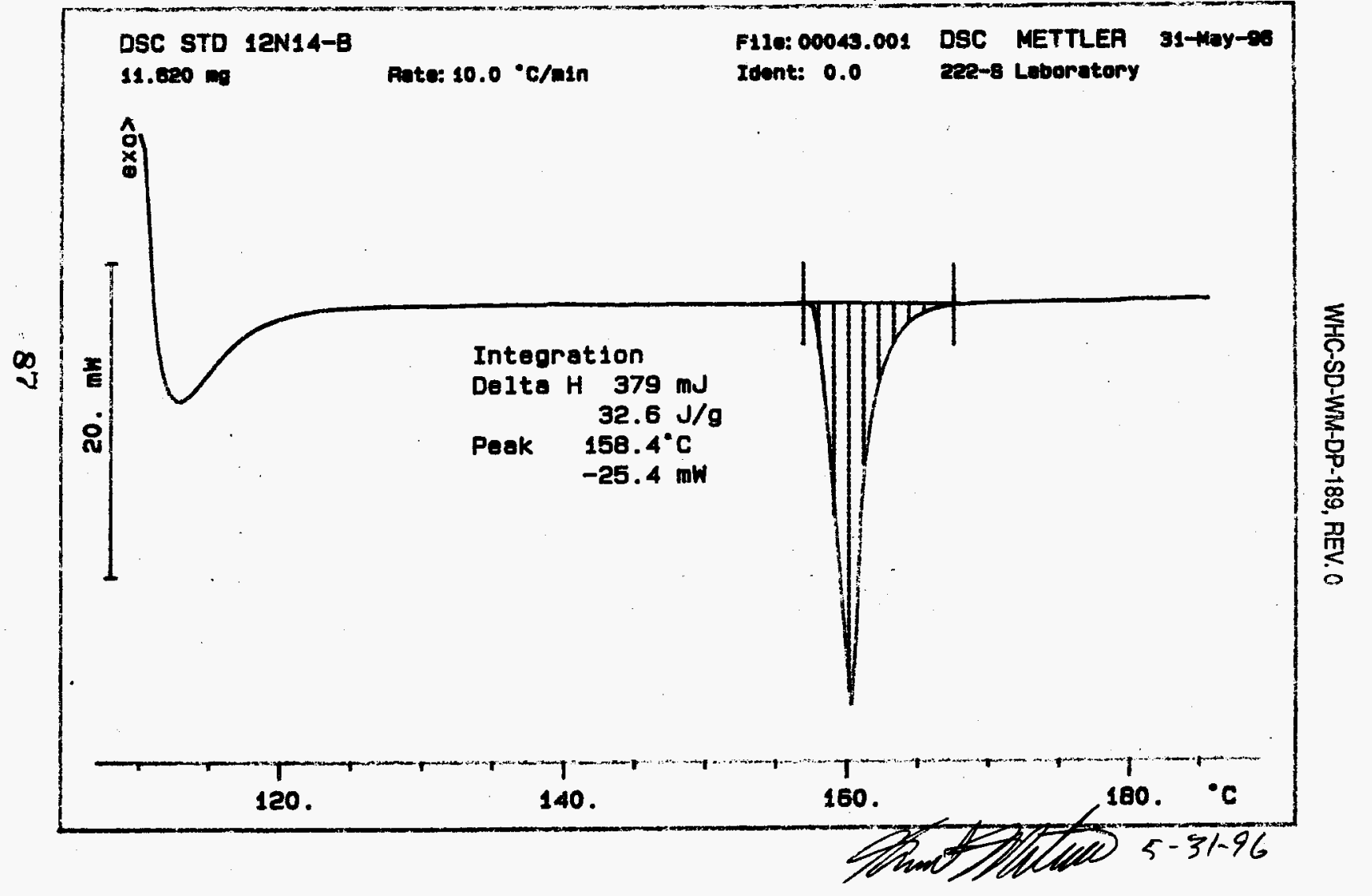




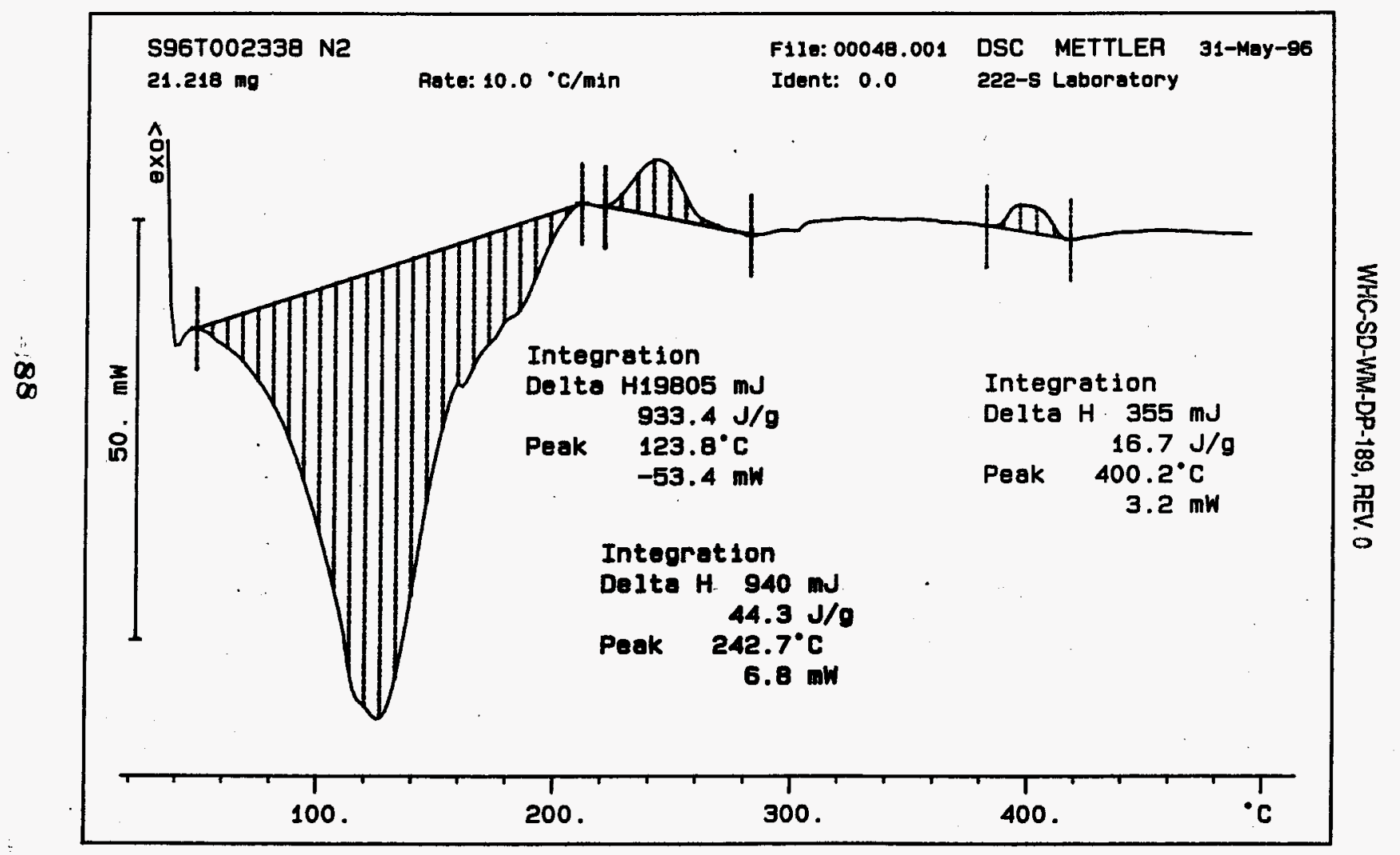




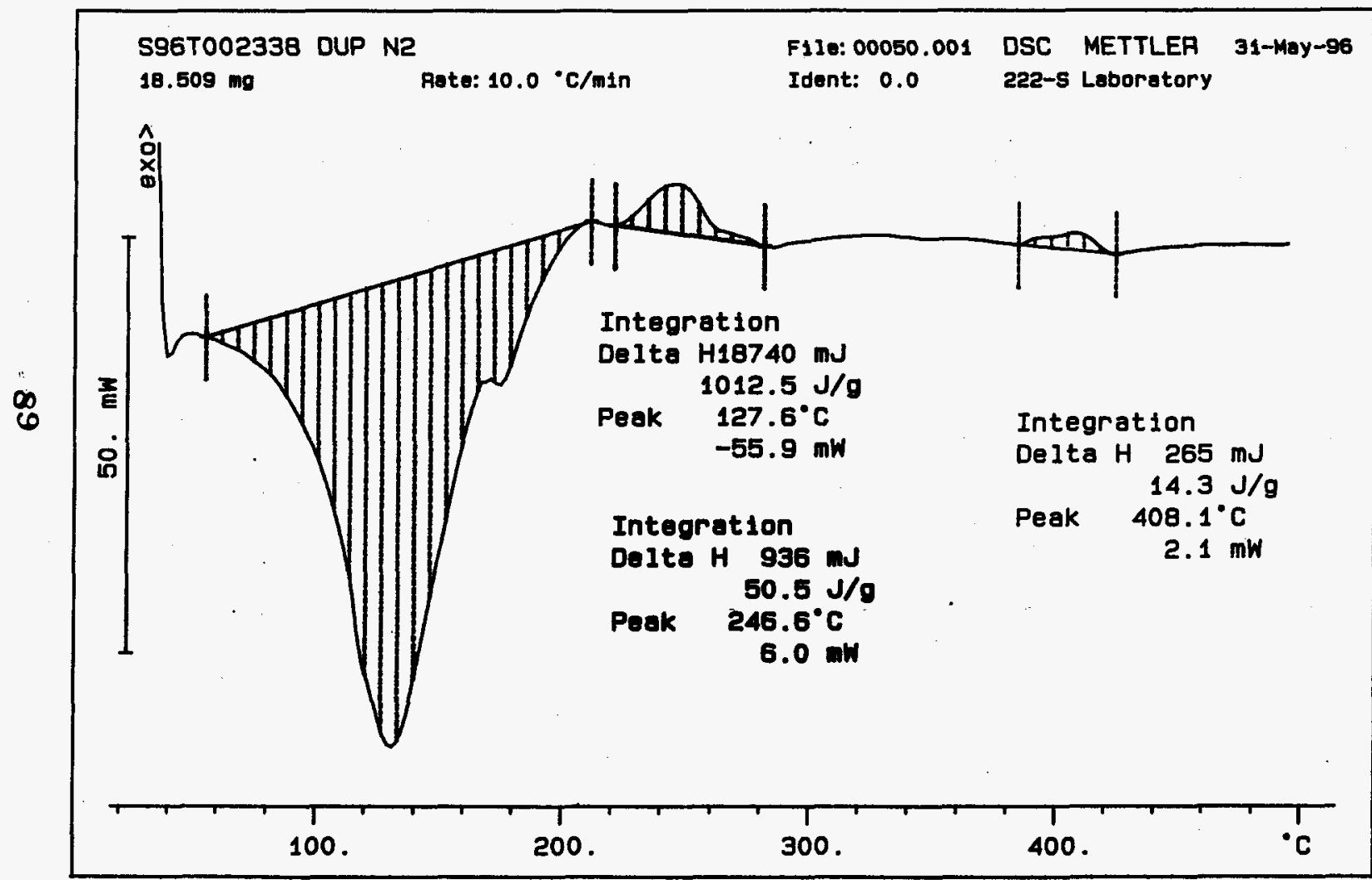

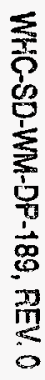




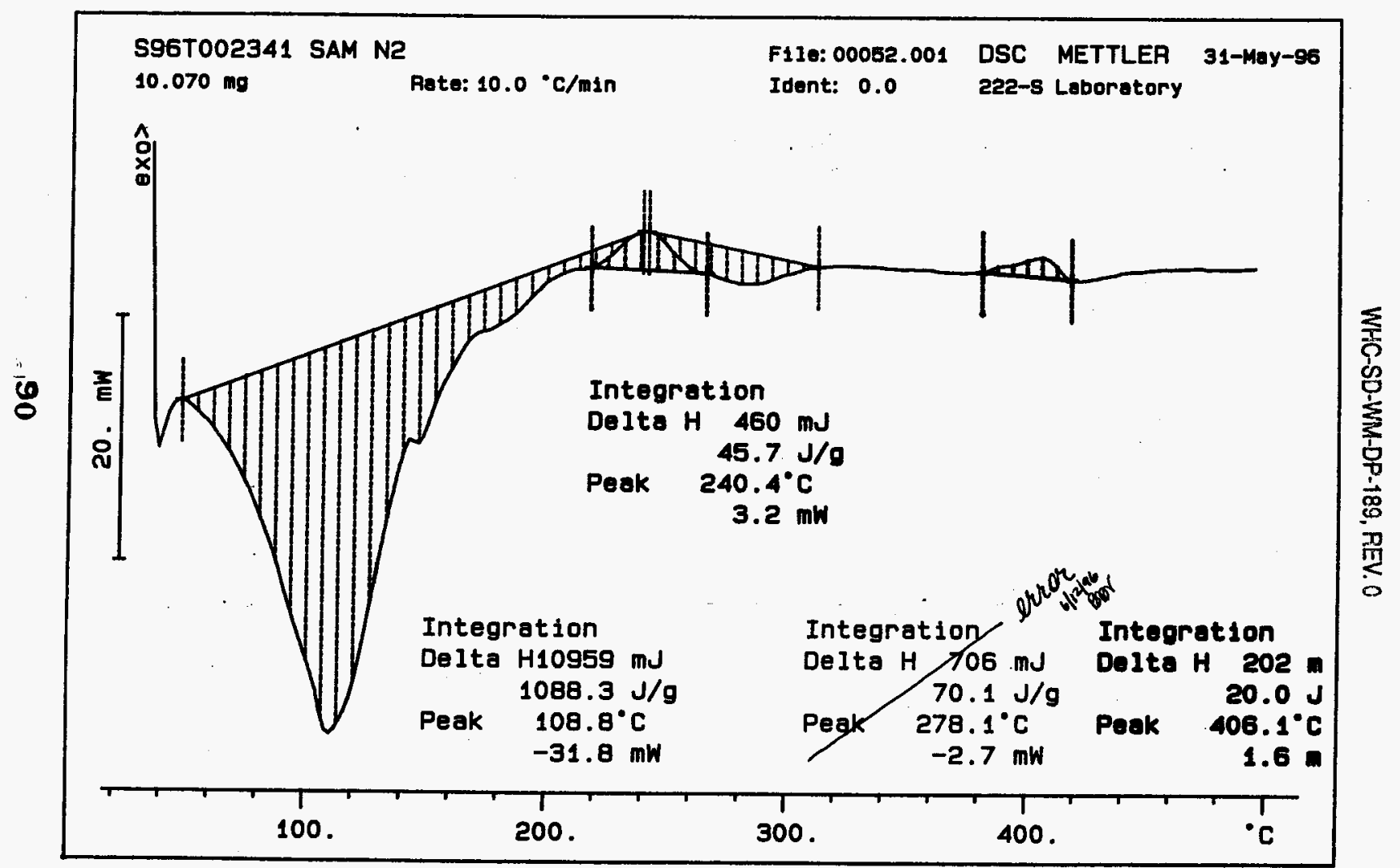




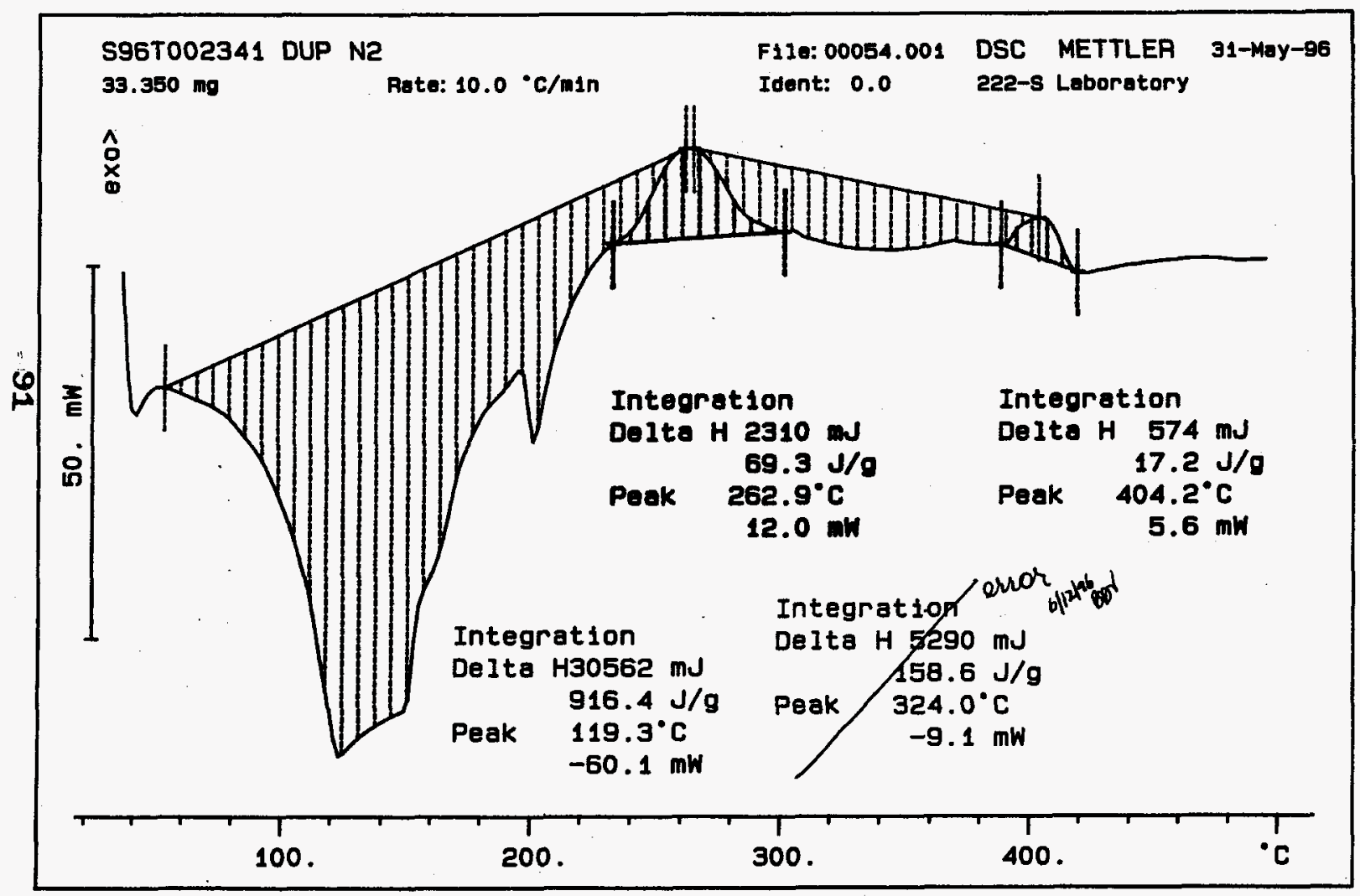

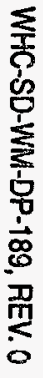


WHC-SD-WM-DP-189, REV. 0

worthistrpt Version $2.105 / 15 / 95$

Page: 1

$06 / 12 / 9615: 39$

LABCORE Data Entry Template for Worklist\#

9261

Analyst: $\quad$ ADP $\quad$ Instrument: DSCO 3 Book \#12N14B

Method: LA-514-114 Rev/Mod C-1

Worklist Comment: U-102 DSC RUN UNDER 'N2. RCJ

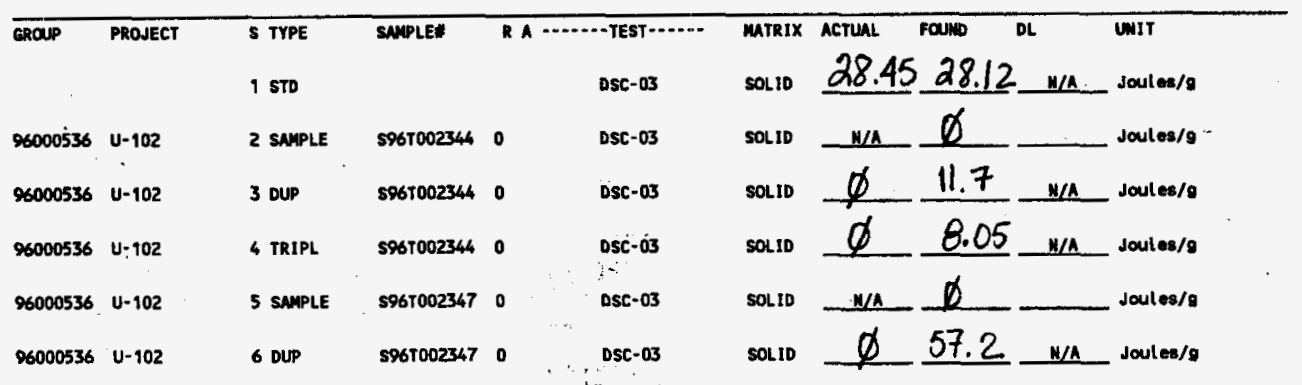

Final page for worklist \# $\quad 9261$

See attached for signatures

Analyst Signature Date, $6-1296$

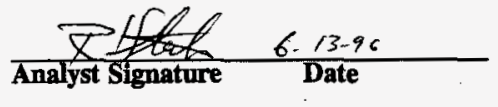

Verified/Vacidated by

Blandina Valenzuela

$6 / 141960$

S96 TOO 2344 was run in triplicate because of the small difference between sample and duplicate.

Data Entry Commons: 9967002347 wa o not run in triplicate because the duplicate result was not close to the notification limit.

Units shown for QC (SPR \& STD) may not reflect the actual units. DL = Detection Limit, $S=$ Worklist Slot Number, $R=$ Replicate Number, $A=$ Aliquot Code.

92 


\section{LABCORE Data Entry Template for Worklist\#}

Analyst: ADP Instrument: DSCO Book\# $12 N / 4 B$

Method: LA-514-113 Rev/Mod

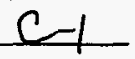

Worklist Comment: U-102 DSC RUN UNDER N2. RCJ

\begin{tabular}{|c|c|c|c|c|c|c|c|c|c|c|}
\hline \multirow[t]{2}{*}{ EROUP } & \multirow[t]{2}{*}{ PRONECI } & S TYPE & \multirow[t]{2}{*}{ SATPLE: } & \multicolumn{2}{|c|}{ R A $\cdots . . .-$-TEST $\cdots$} & \multirow{2}{*}{$\begin{array}{l}\text { MATRIX } \\
\text { SOLID }\end{array}$} & \multirow[t]{2}{*}{ ACTunL } & \multirow[t]{2}{*}{ Found } & DL & \multirow{2}{*}{$\begin{array}{l}\text { UNIT } \\
\text { Joules/s }\end{array}$} \\
\hline & & 1 sto & & & Dsc-01 & & & & $\mathbf{n} / \mathbf{A}$ & \\
\hline 96000536 & $U-102$ & 2 SNMPLE & $596 r 002344$ & 0 & DSC-01 & sol.10 & $M / \mathbf{a}$ & & & Joules/s \\
\hline 96000536 & $U-102$ & 3 DUP & 596ro02344 & 0 & Dsc-01 & SOLID & & & $N / A$ & Joules/s \\
\hline 96000536 & $U-102$ & 4 SNMPLE & $\$ 967002347$ & 0 & DSC-01 & SOLID & $N / \mathbf{A}$ & & & Joules/g \\
\hline 96000536 & U-102 & 5 DuP & 5961002347 & 0 & Dsc-01 & SOL 10 & & & $\mathbf{N} / \mathbf{A}$ & Jouted/a \\
\hline
\end{tabular}

Final page for worklist \# $\quad 9261$

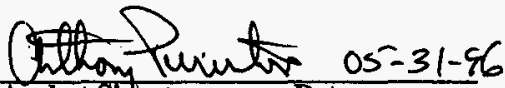

Analyst Sighature Date

\section{Analyst Signature Date}

DSC-03 instrument

was used.

$6-12-96$

Blandina

Valenguela

Dạta Eutry Comments:

Units shown for $Q C$ (SPR \& STD) may not reflect the actual units. $D L=$ Detection Limit, $S=$ Worklist Slot Number, $R=$ Replicoste Number, $A=$ Aliquot Code. 
Curve 1: DSC

F1le info: IND053101 Fr1 May 31 15: 11: 171996

Sanple Veight: 12.080 . ng

12N14-8 INDILM AT 10CWMIJGNATURE BELOW REPRESENTS CHEMICAL TECHNOLOGIST/CHEMIST THAT

COMPLETED/VERIFIED THE CALIBRATION/ANALYSIS ON PAGES 94 TO 99.

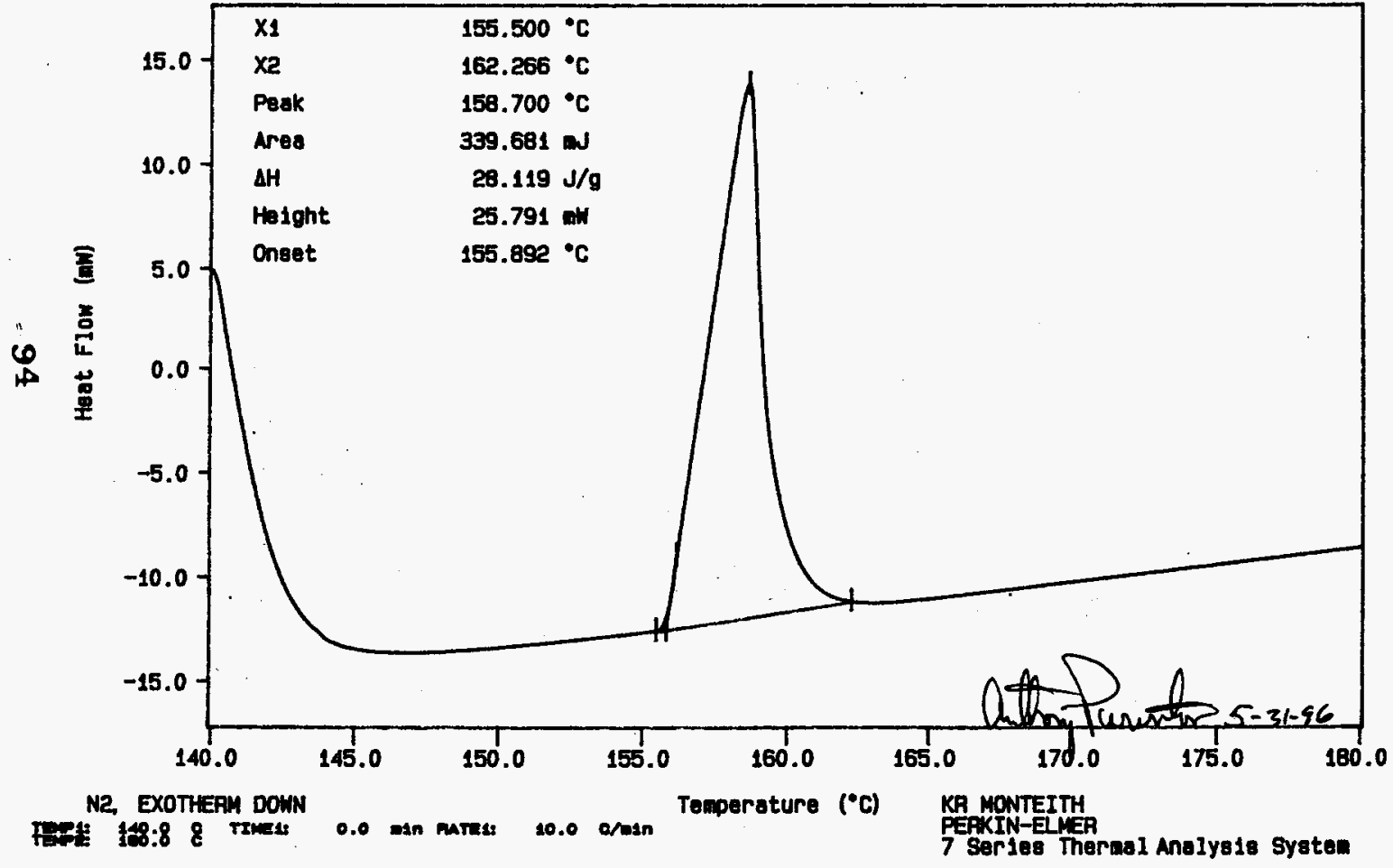


Curve 1: DSC

File info: SAM053101 Fri May 31 19: $15: 431996$

Sanple Weight: 13.100

ing

S96T002344 SAM

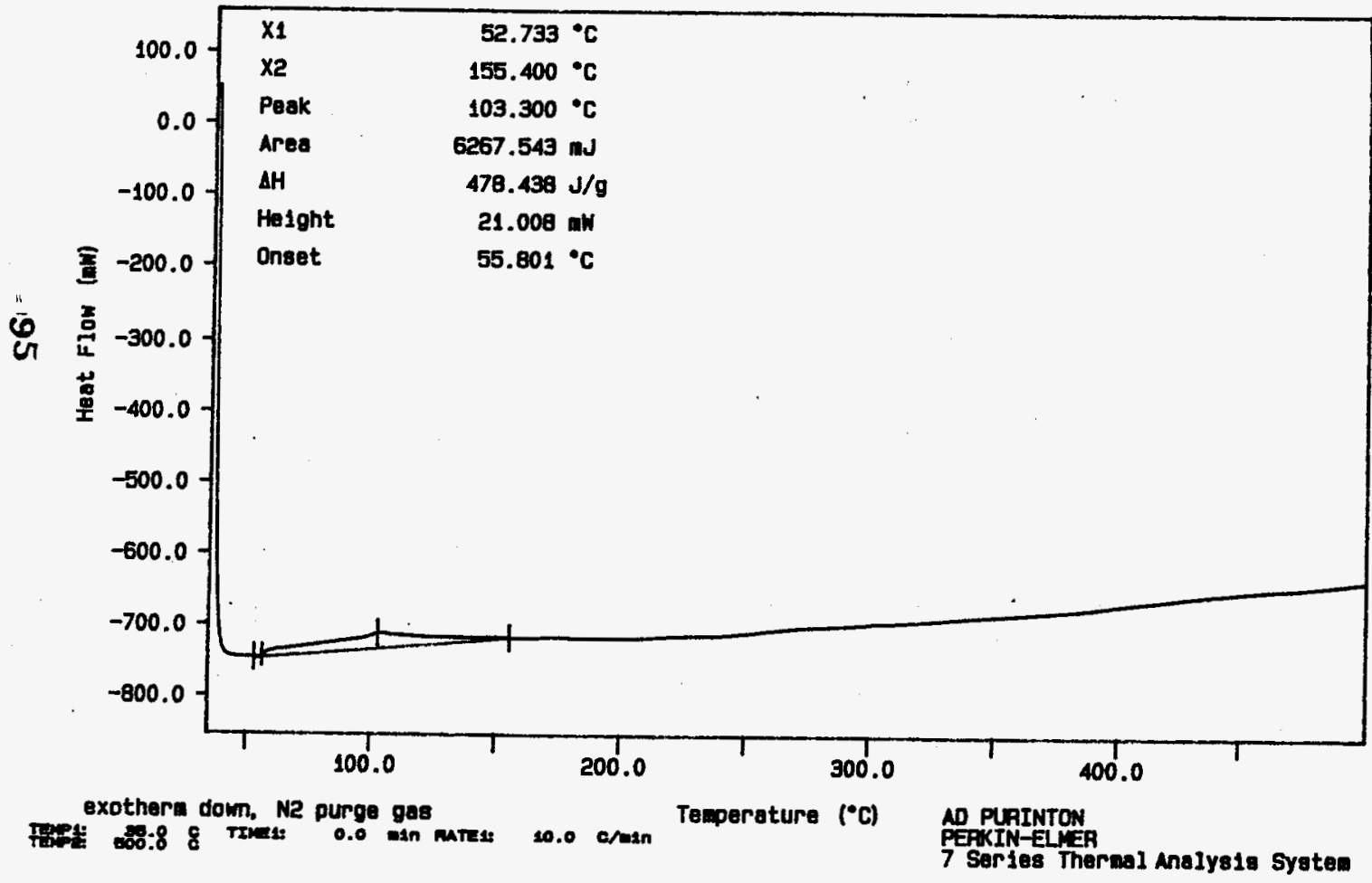


- Curve 1: DSC

F1le info: SAM053102 Fri May 31 21: 59: 101996

Saaplo Ne1ght: 46.410

ang

S96T002344 DUP

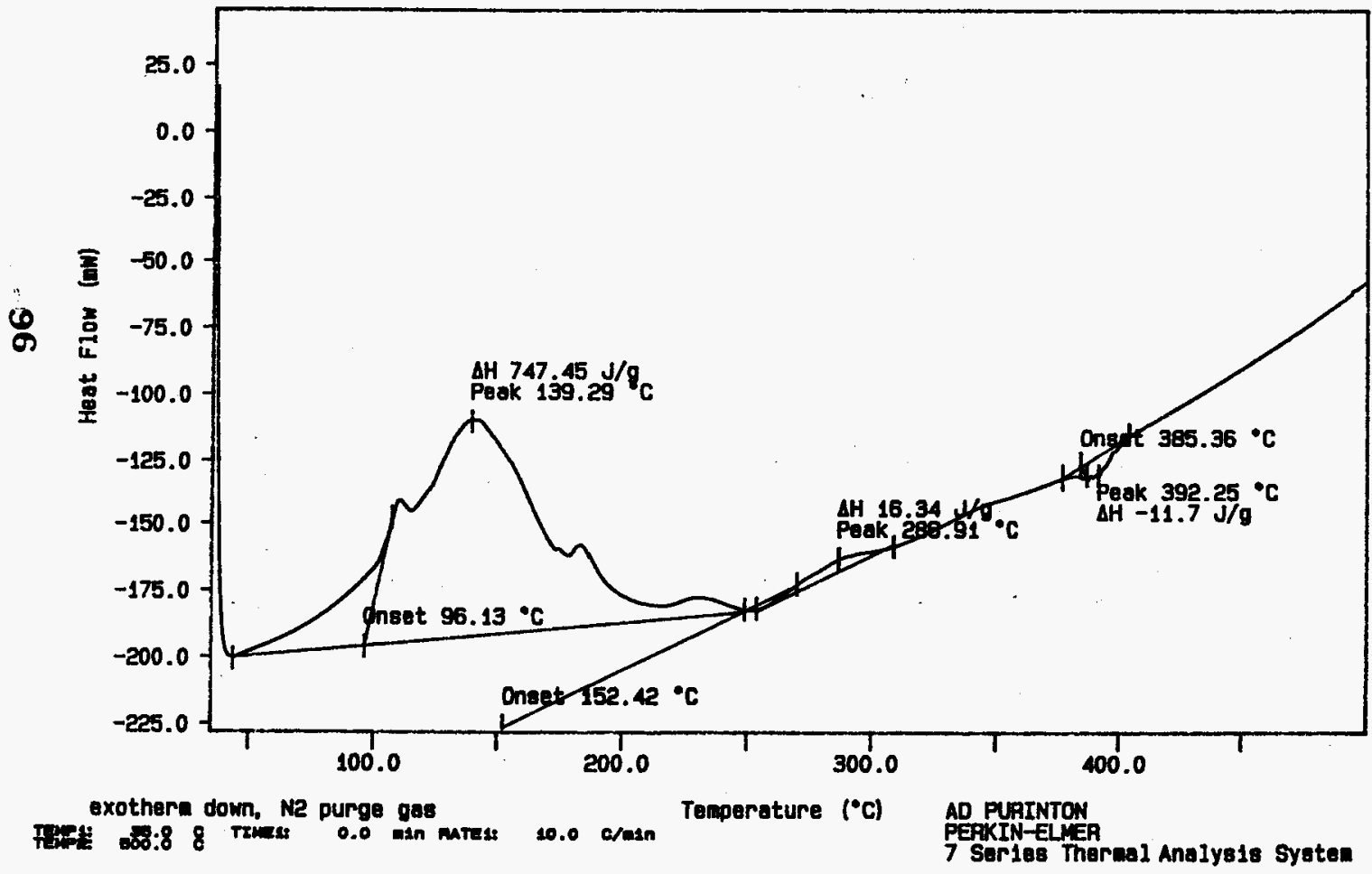


Curve 1: DSC

Ftle info: SuM053105 Sat wn 1 02 35: 491996

Sample Neight: $40.240 \mathrm{ng}$

S96T002344 TRP

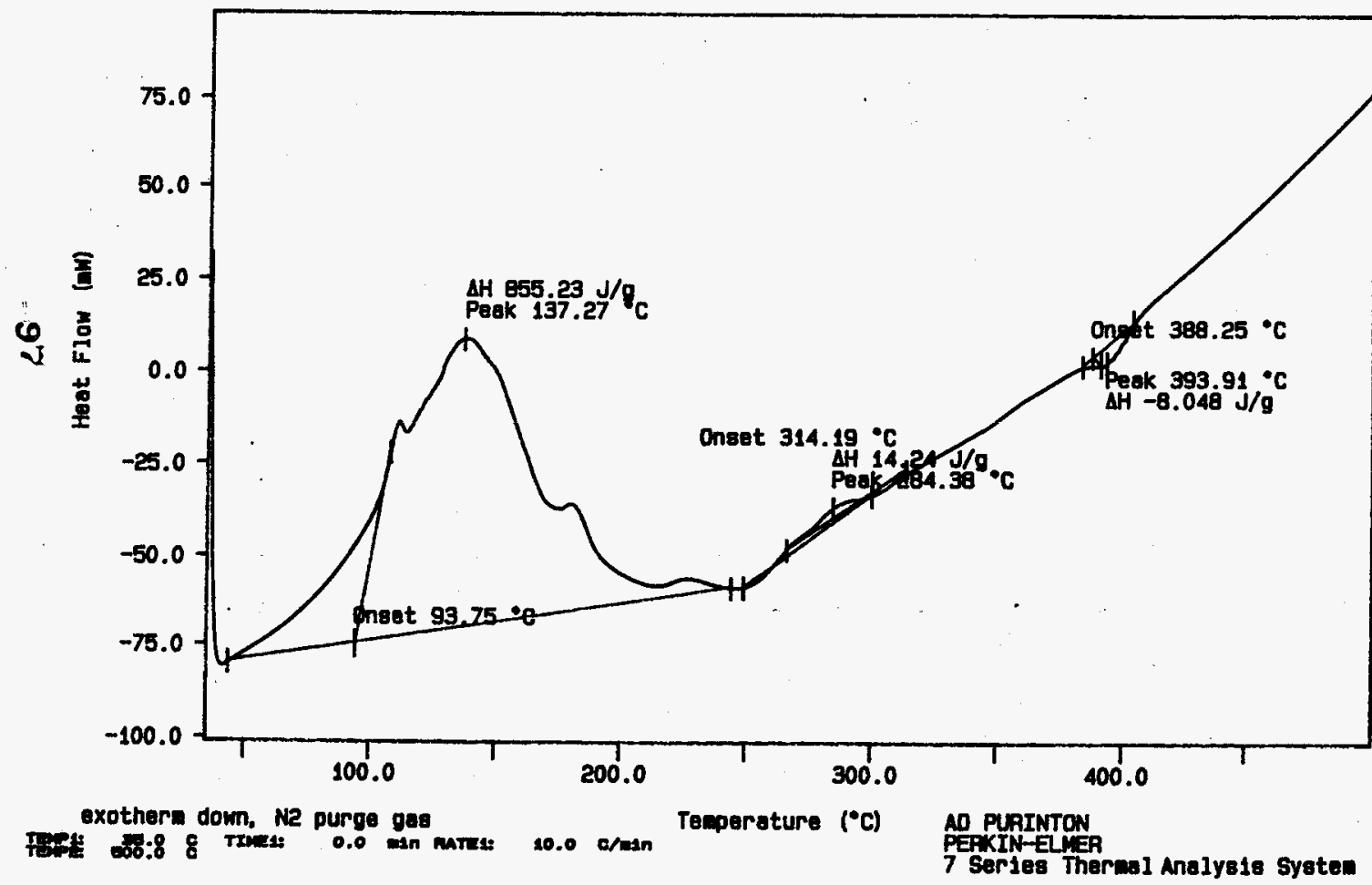


Curve 1: DSC

F1le Info: SAM053103 Fr1 May 31 29: 17: 441996

Sample Weight: 41.070

S96T002347 SAM

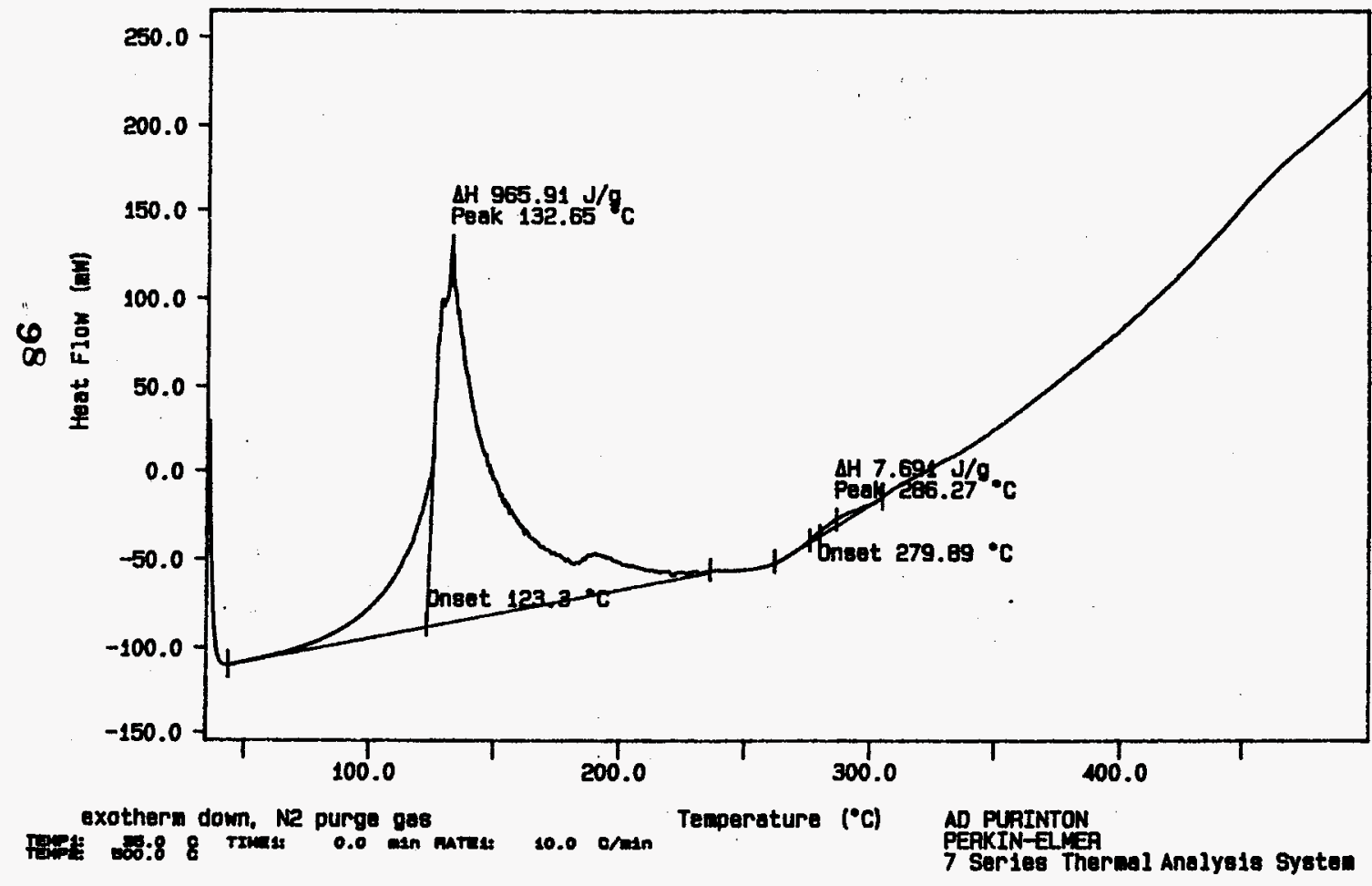


Curve 1: DSC

F1le 1nfo: SAM053104 Sat tun 1 01: 28: 251996

Sample Waight: 39.200

S96T002347 DLP

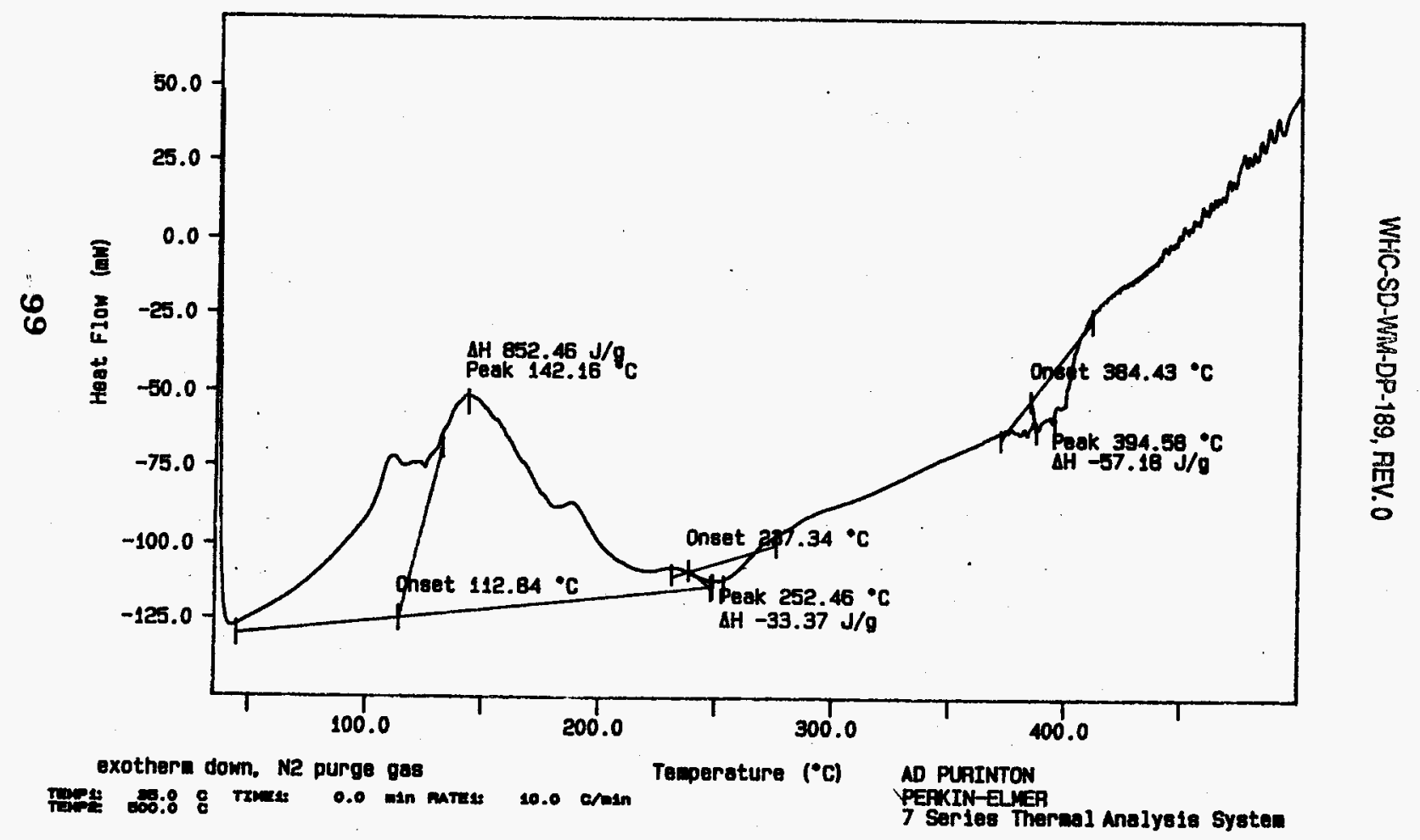


wortlistrpt Version 2.1 05/15/95

05/31/96 13:10

WHC-SD-WM-DP-189. DEF

Page: I

LABCORE Data Entry Template for Worklist\#

9381

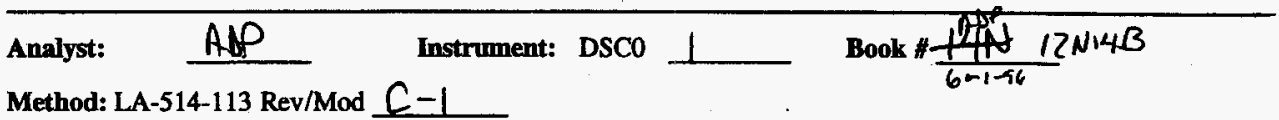

Worklist Comment: U-102 DSC RUN UNDER N2. RCJ

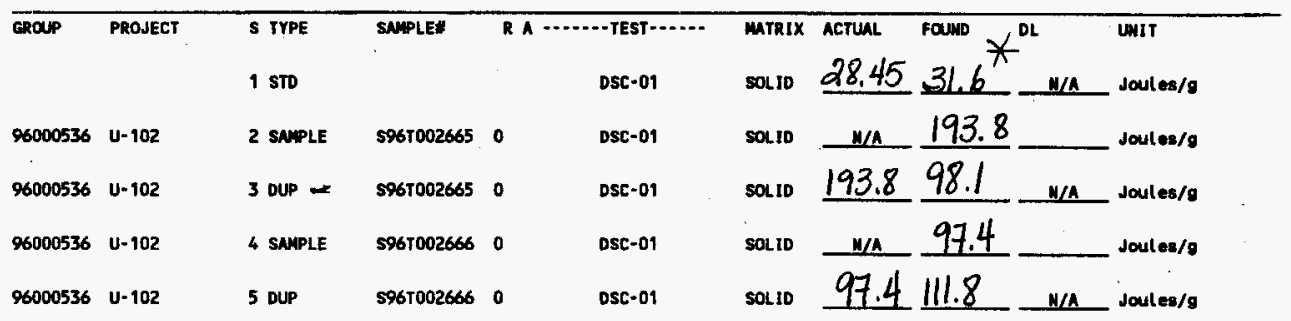

Final page for worklist \# 9381

Chanflunth 6-1-96

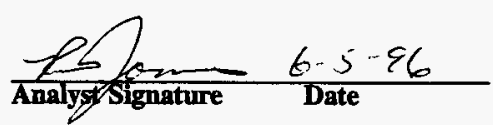

Validated by Hanasts b-6-96

S96T002665 Sample will be rerun due to high RPD's and the thermograms did not $100 \mathrm{~K}$ similar.

Data Entry Commons: S967002666 Samples were mot rerun because The RPD's wee close to 10\% and the results are not close to the notification limit.

Units shown for $Q C$ (SPK \& STD) may not reflect the actual units. $D L=$ Detection Limit, $S=$ Worklist Slot Number, $R=$ Replicate Number, $A=$ Aliquot Code.

100 


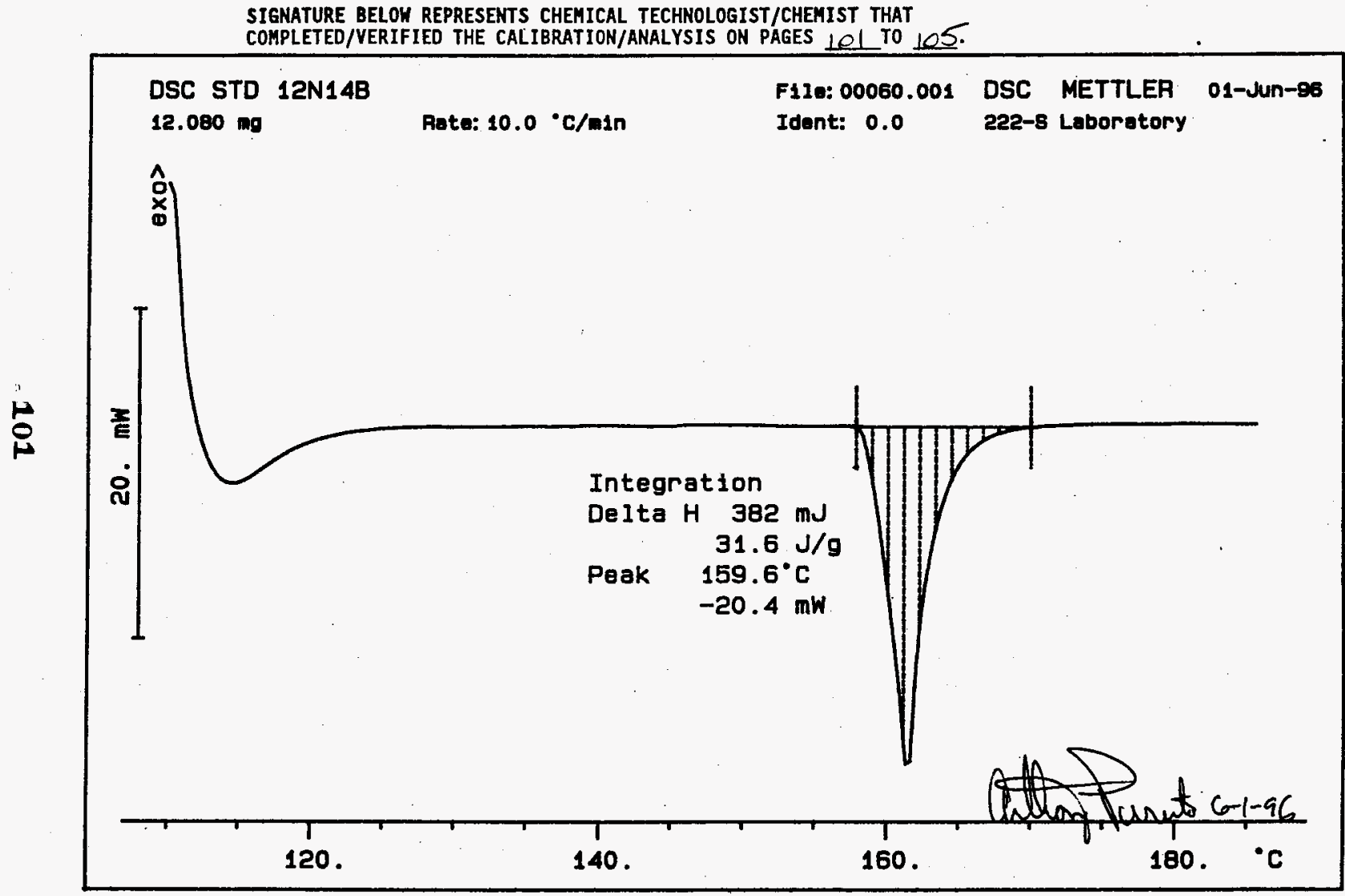


WHC-SD-WM-DP-189, REV. 0

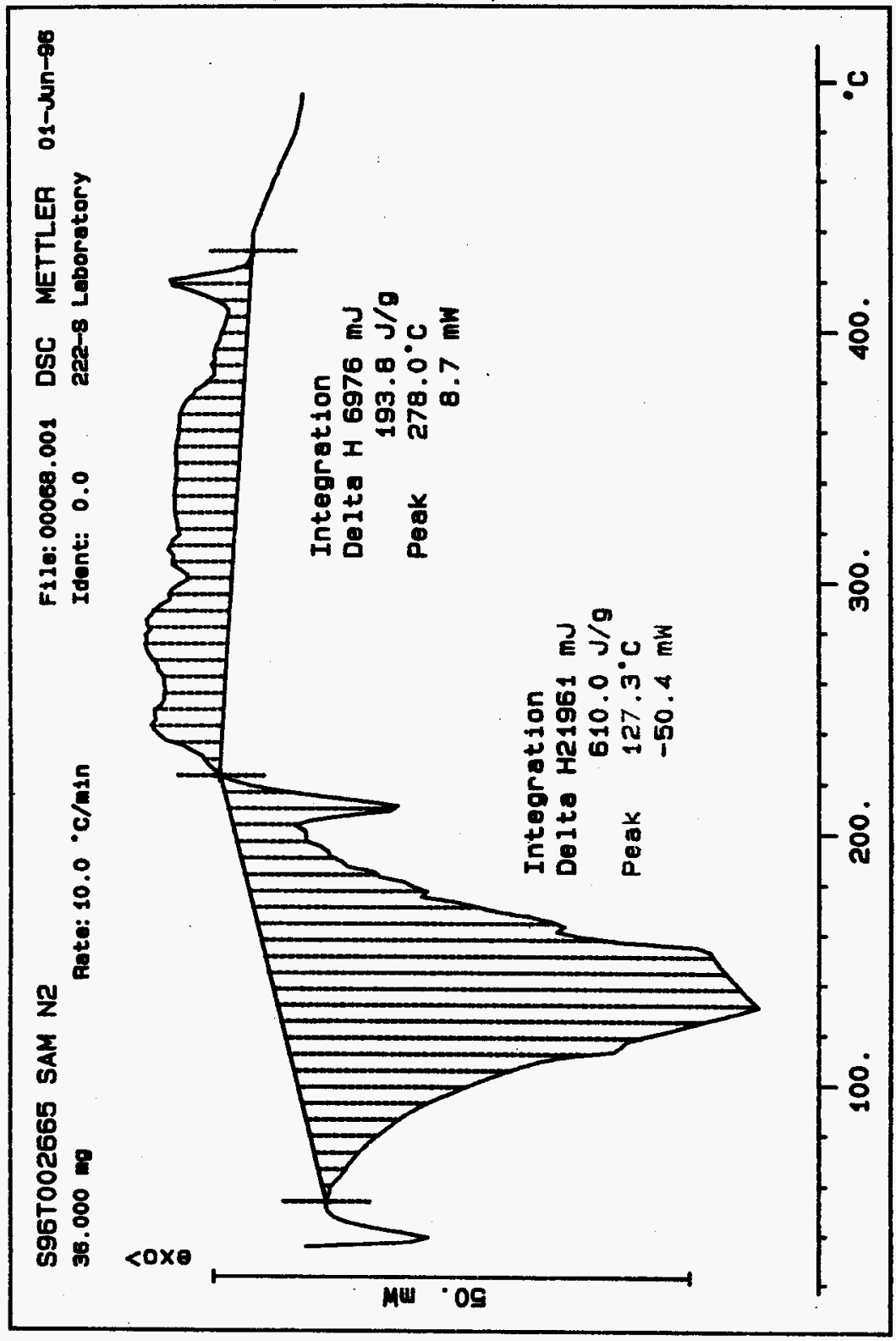




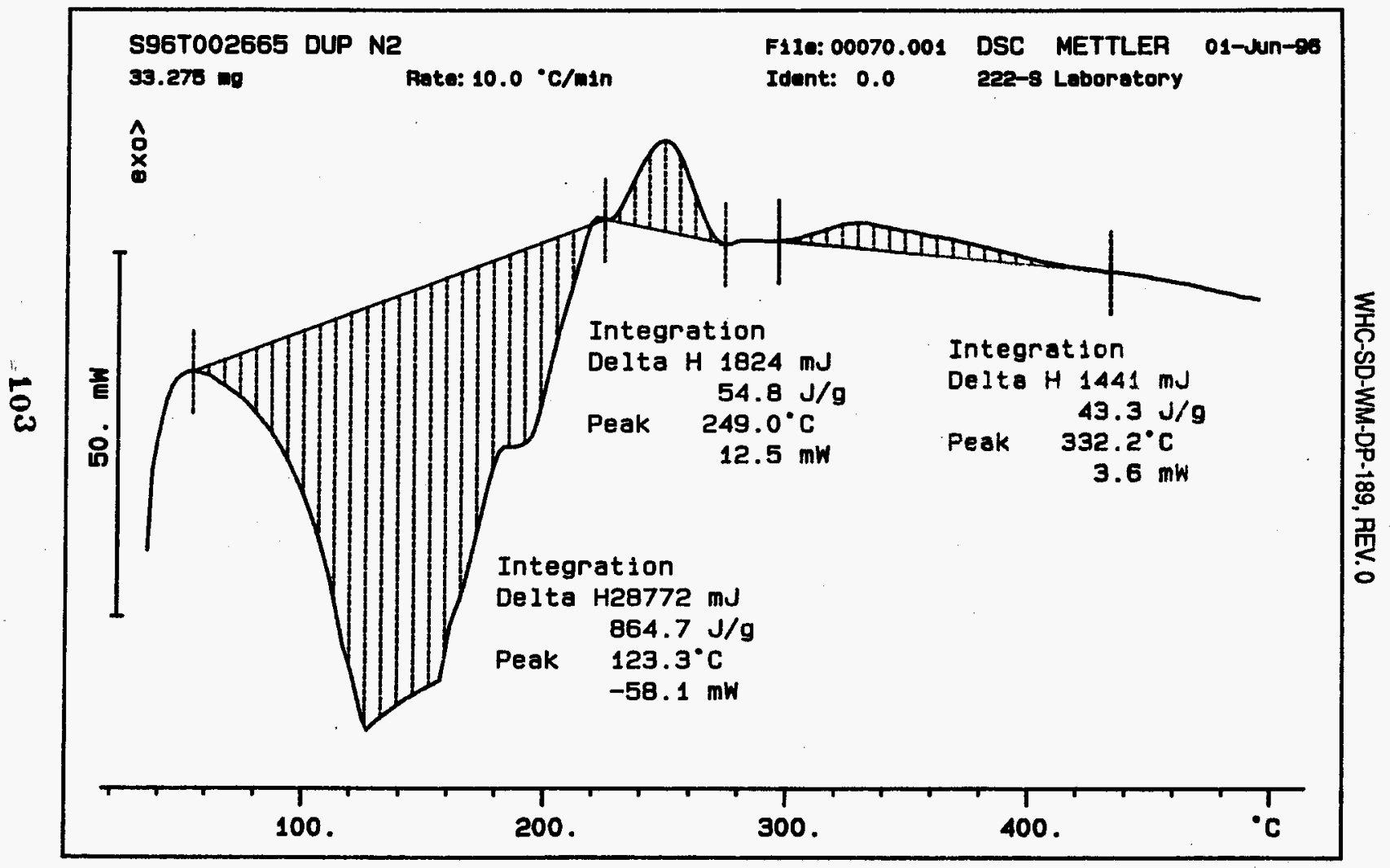




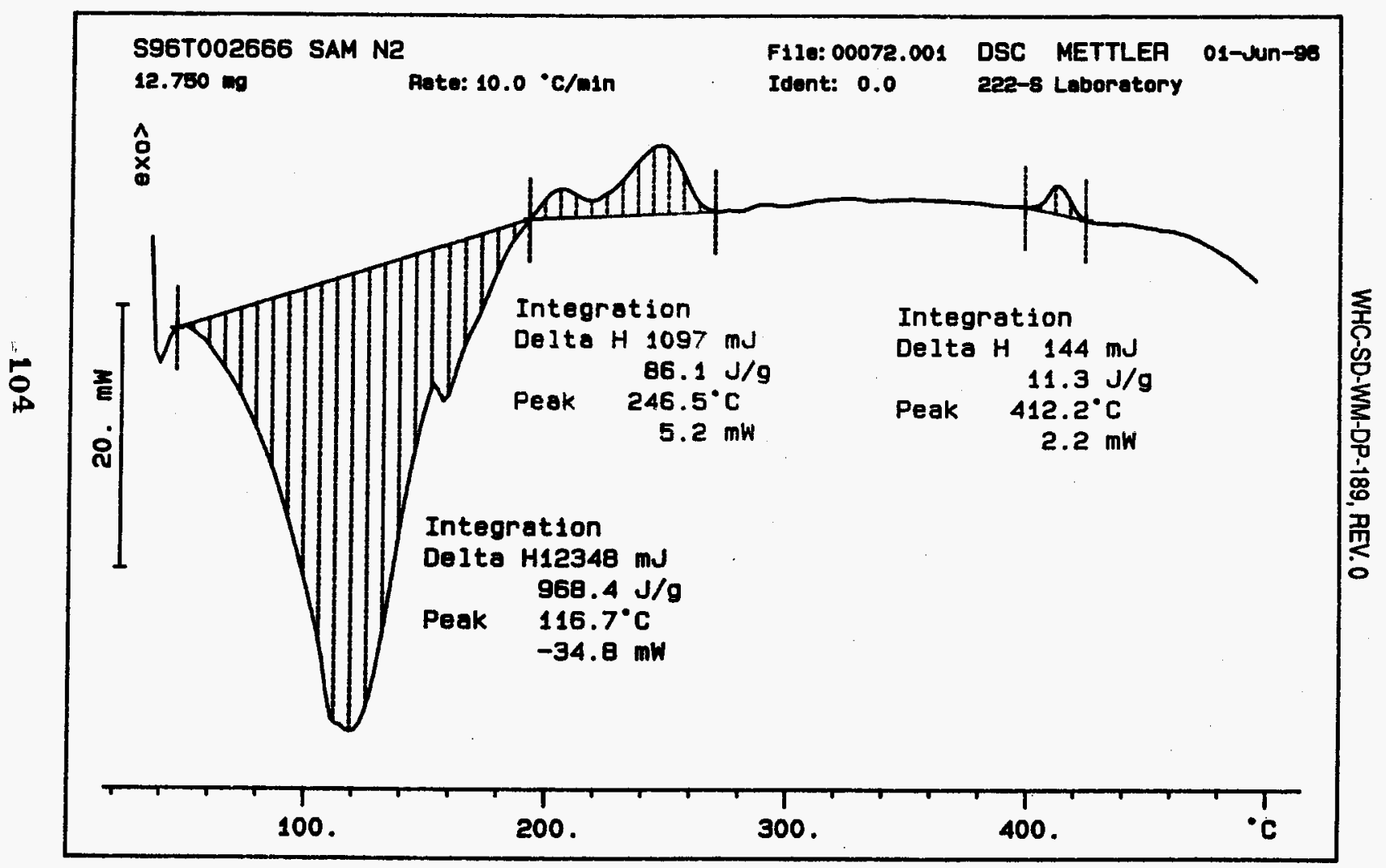




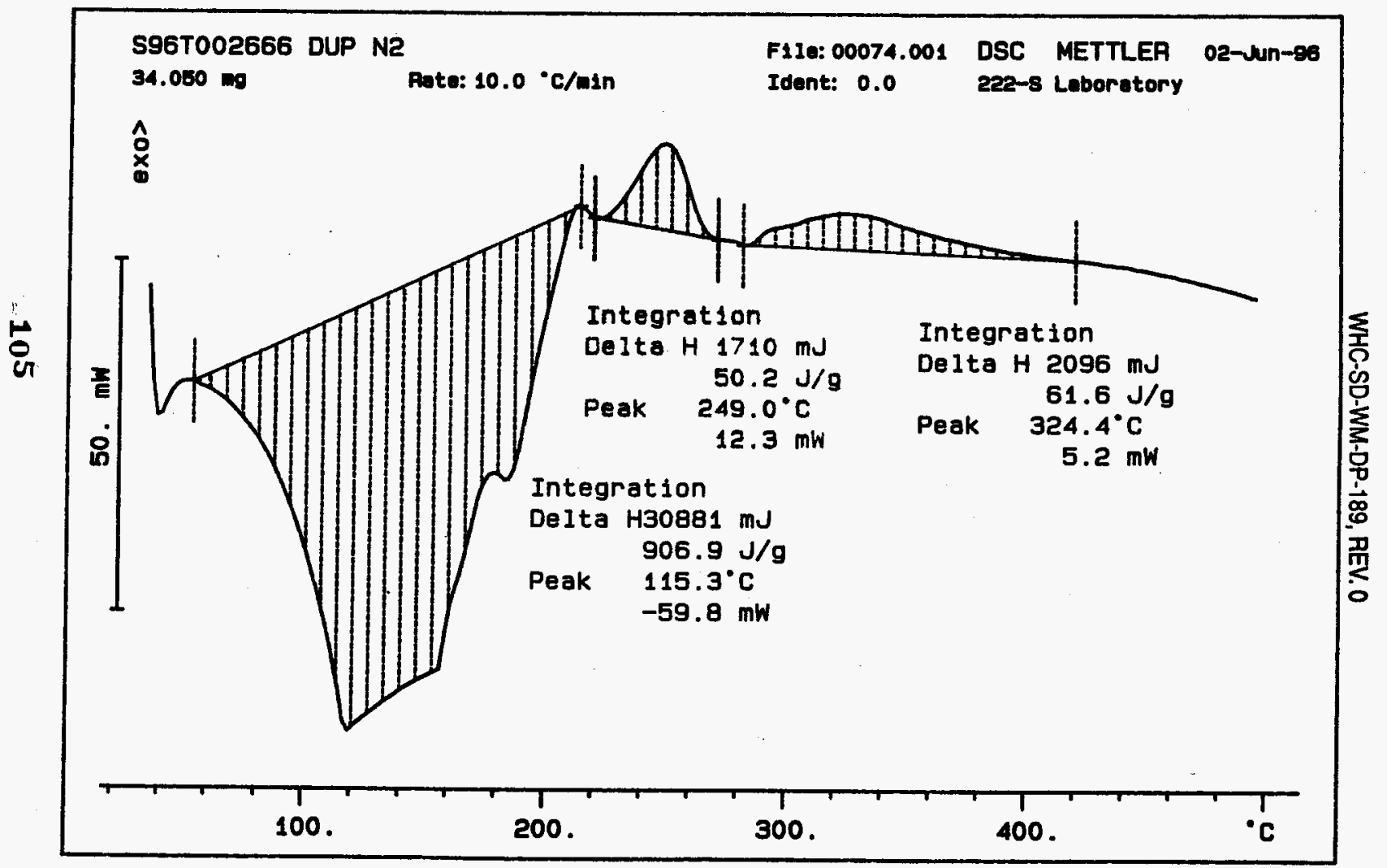


morklistrpt Version 2.1 05/15/95

05/31/96 13:11

WHC-SD-WM-DP-189, REV. 0

LABCORE Data Entry Template for Worklist\#
Page: 1

9382

Method: LA-514-113 Rev/Mod C -

Worklist Comment: U-102 DSC RUN UNDER N2. RCJ

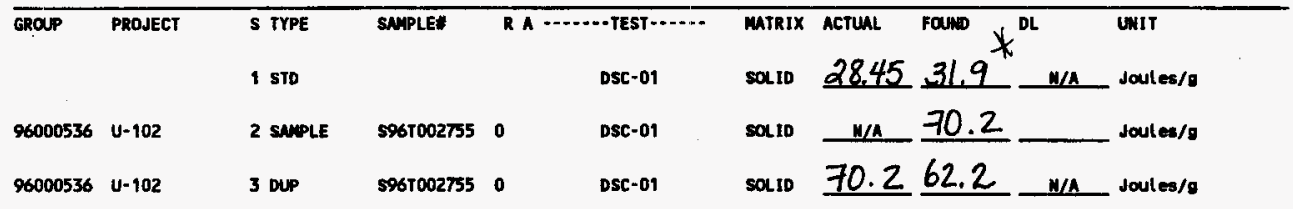

Final page for worklist \# 9382
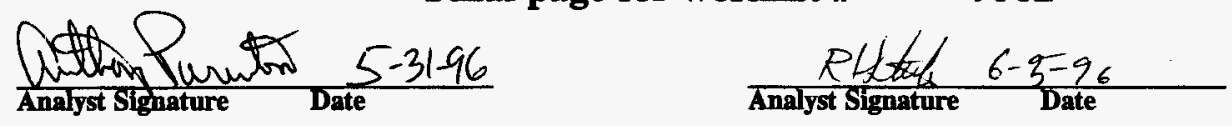

Validated by Hon aston 6.6.96

Data Bray Commons: Sample results are the sum of two exotherms.

Units shown for $Q C$ (SPK \& STD) may not reflect the actual writs. DL = Detection Limit, $S=$ Worklist Slot Number, $R=$ Replicate Number, $A=$ Aliquot Code.

106 


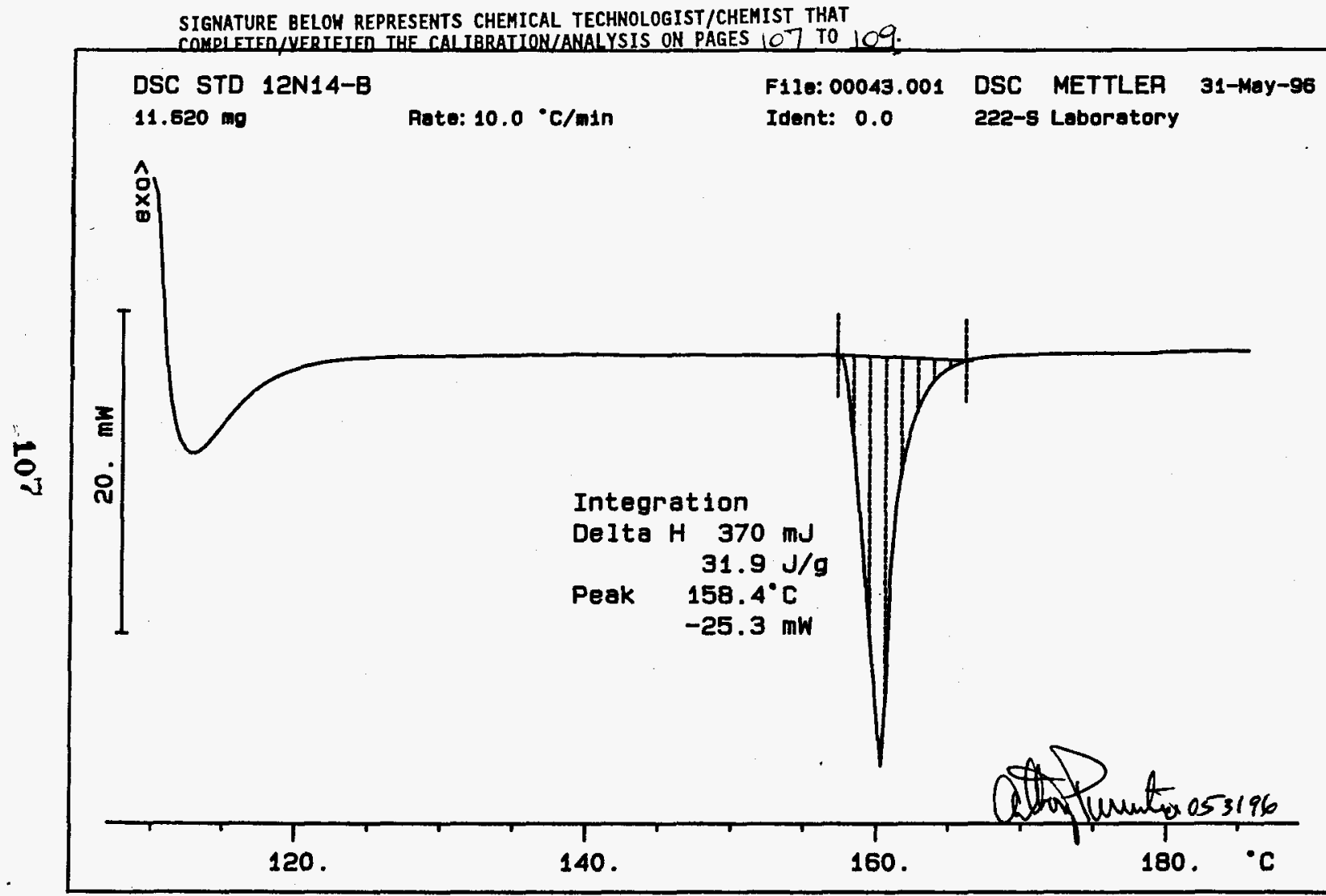




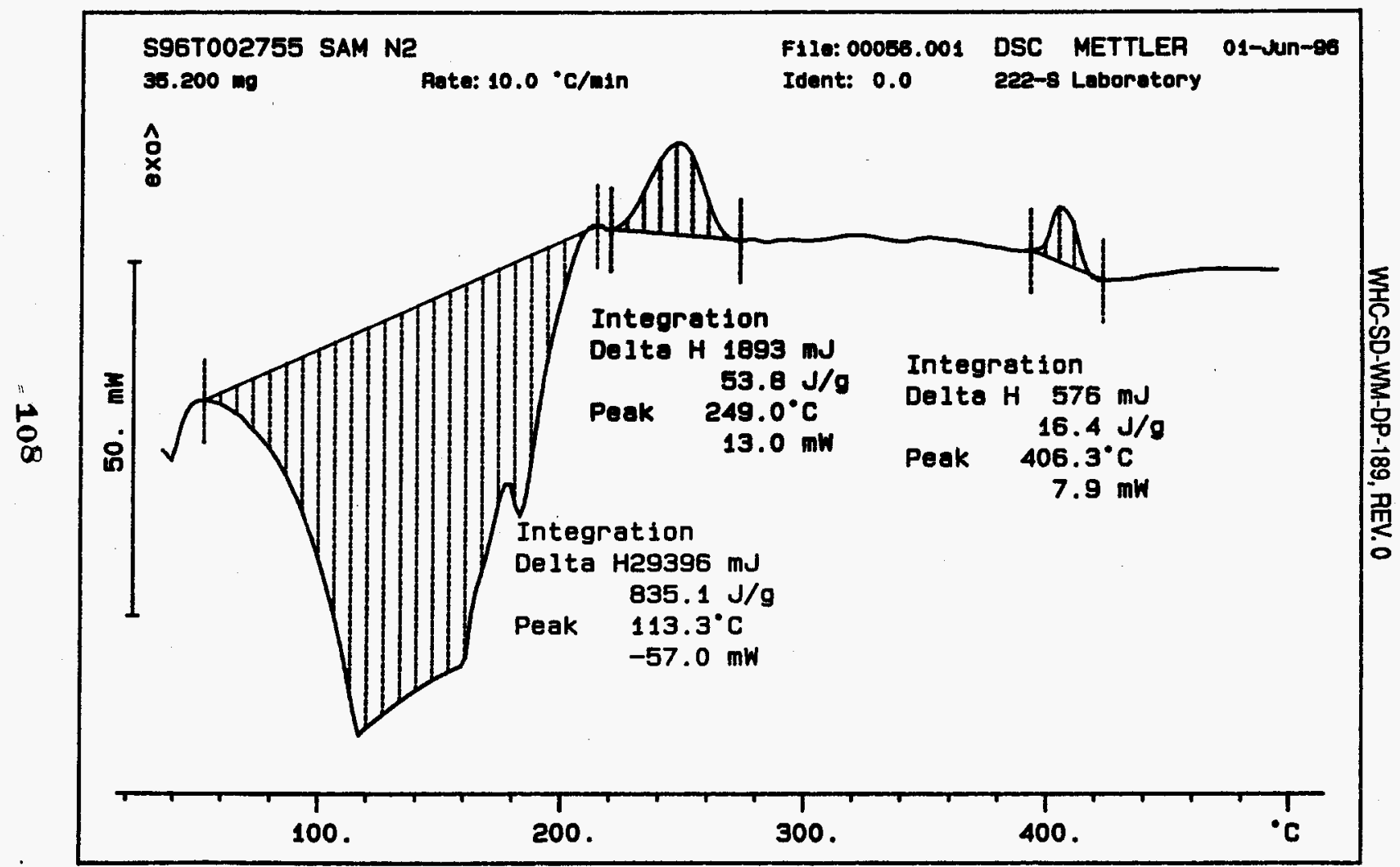




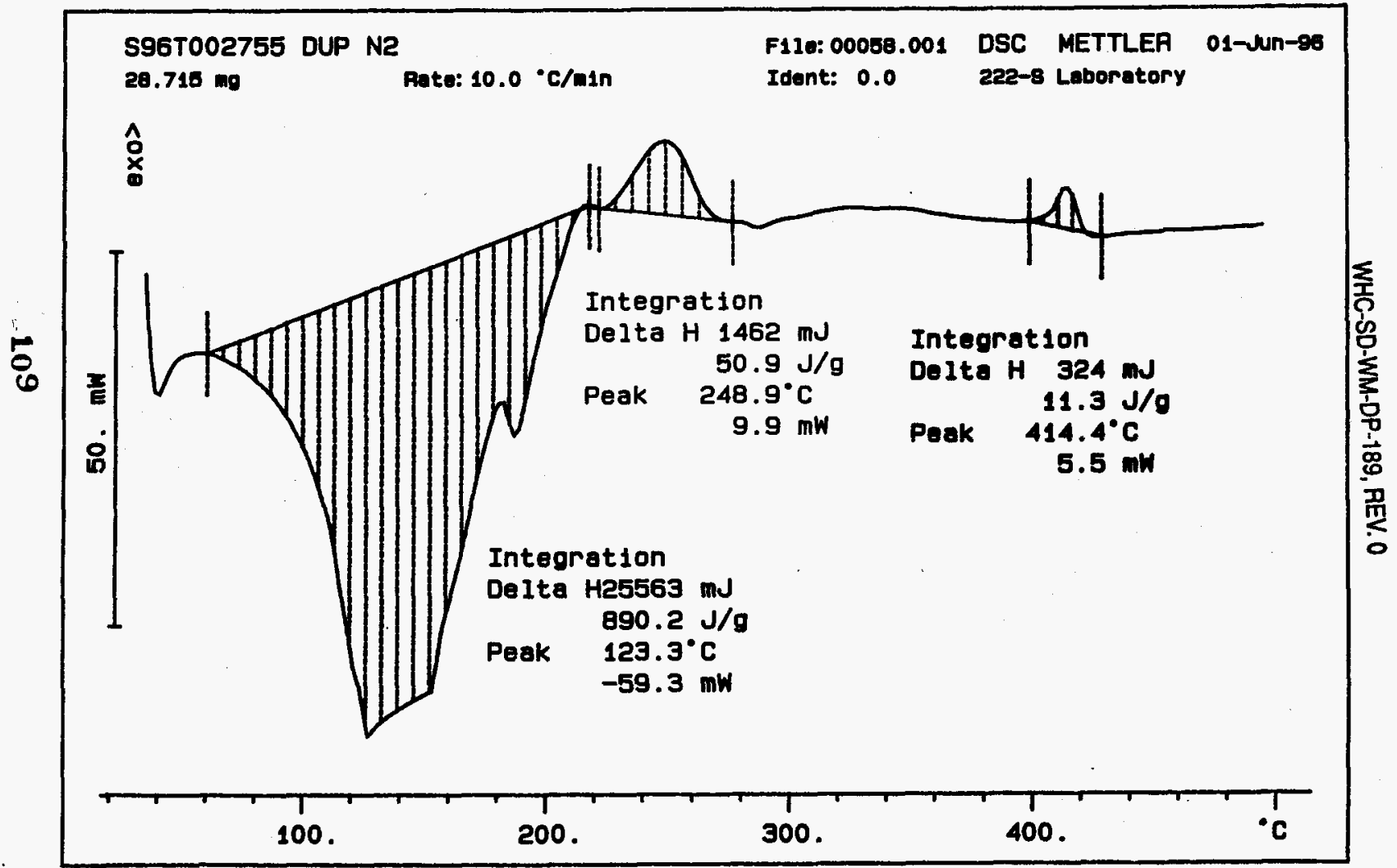




Analyst: $D C D$ Instrument: $D S C 011$ Book \# $12 N / 4 B$

Method: LA-514-113 Rev/Mod C-1

$102 \mathrm{Men} b / 2 / 99$

Worklist Comment: U-108 DSC RUN UNDER N2. RCJ

\begin{tabular}{|c|c|c|c|c|c|c|c|c|c|c|c|}
\hline \multirow[t]{2}{*}{ choup } & \multirow[t]{2}{*}{ PROJECT } & S TYPE & SAYPLE" & RA & 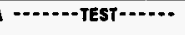 & MATRIX & ACTUAL & FOUND & DL & \multirow{2}{*}{\multicolumn{2}{|c|}{ Len IT }} \\
\hline & & 1 STO & $\cdot$ & & DSC-01 & SOLID & & & & & \\
\hline 96000536 & U-102 & 2 SNPLE & 5961002500 & 0 & DSC-01 & SOLID & $\mathbf{N} / \mathbf{A}$ & & & Joules/s & * \\
\hline 96000536 & $U-102$ & 3 DUP & 5961002500 & $\mathbf{0}$ & DSC-01 & SOLID & & & & Joules/o & \\
\hline 96000536 & $U-102$ & 4 SANPLE & S96T002501 & 0 & Dsc-01 & SOLID & $\mathbf{N} / \mathbf{A}$ & & & Joul es/g & \\
\hline 96000536 & $u-102$ & 5 DUP & S96T002501 & 0 & Dsc-01 & SOLJD & & & & Joul 1 s/g & \\
\hline
\end{tabular}

\section{Final page for worklist \# 9459}

\section{$\frac{D_{\text {Analyst Signature }}(x)}{\text { Date }} 6-4-x$}

Verified Validated bry Blandina Valentuela 6/2/96

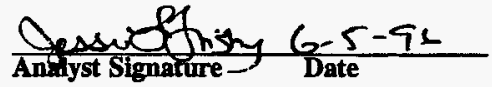

efetwe 6-11-96

Data Entry Comments:

Units shown for $Q C$ (SPK \& STD) may not reflect the actual writs. $D L=$ Detection Limit, $S=$ Worklist Slot Number, $R \cdot=$ Replicate Number, $A=A$ Aliquot Code. 


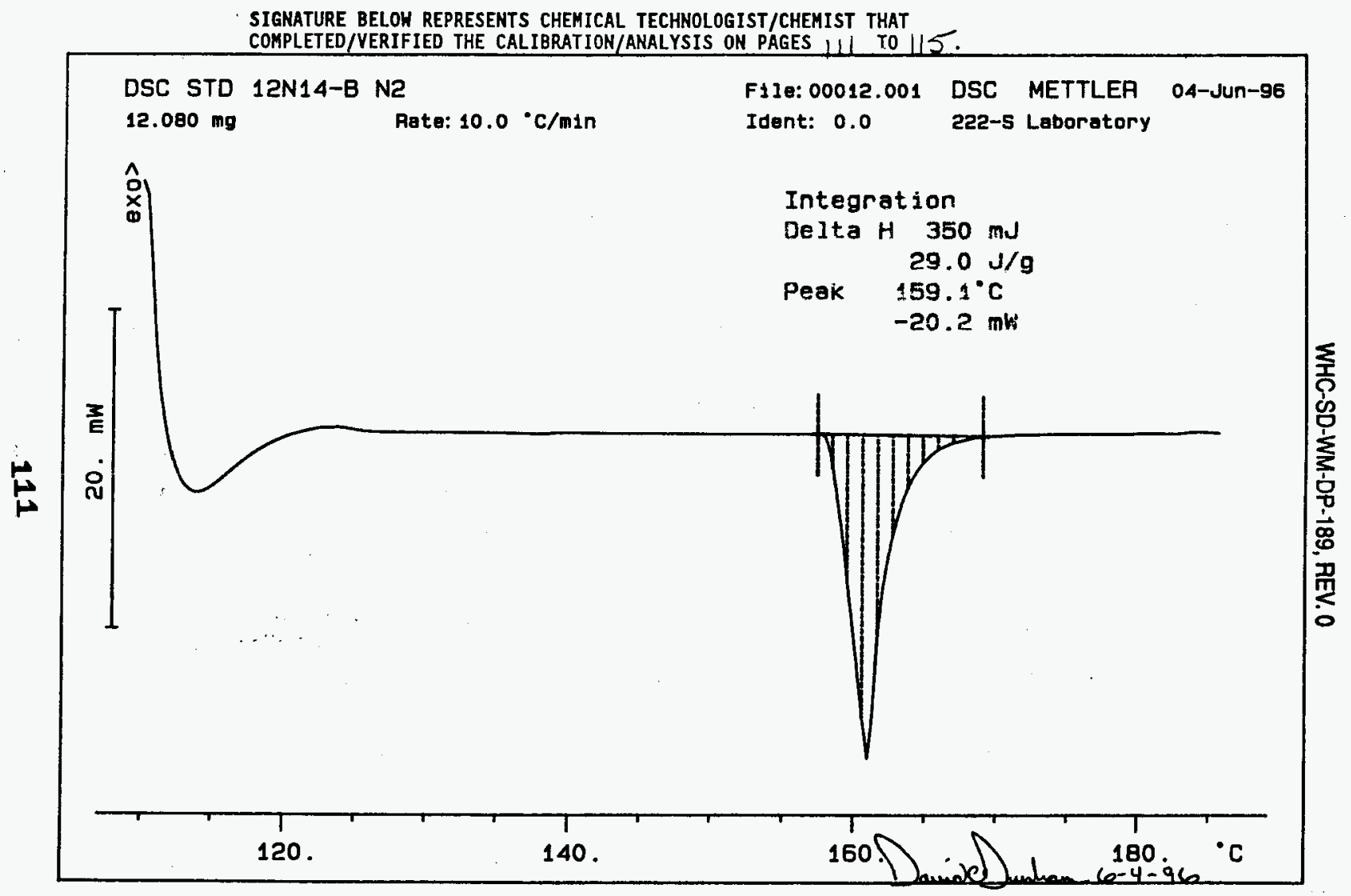




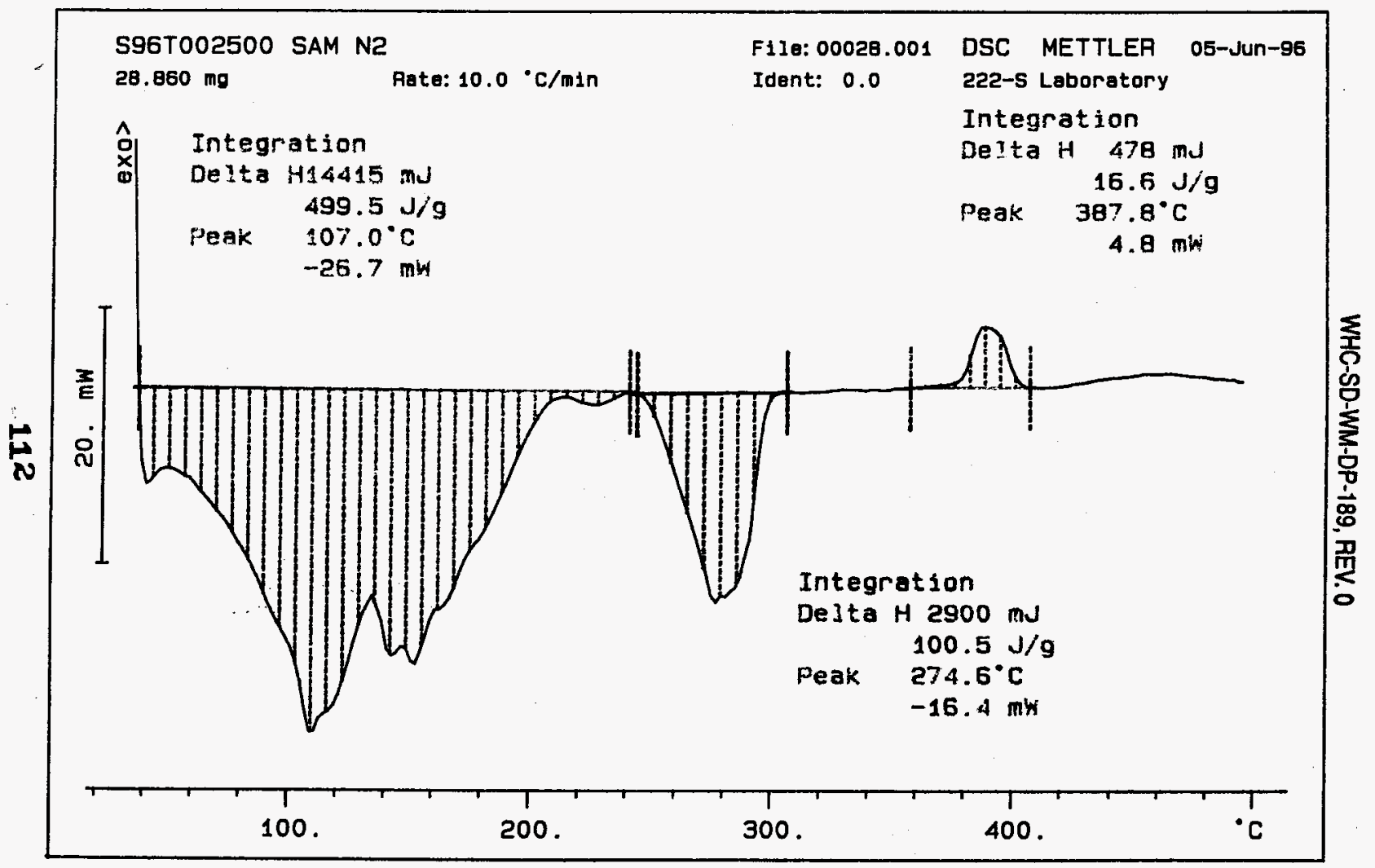




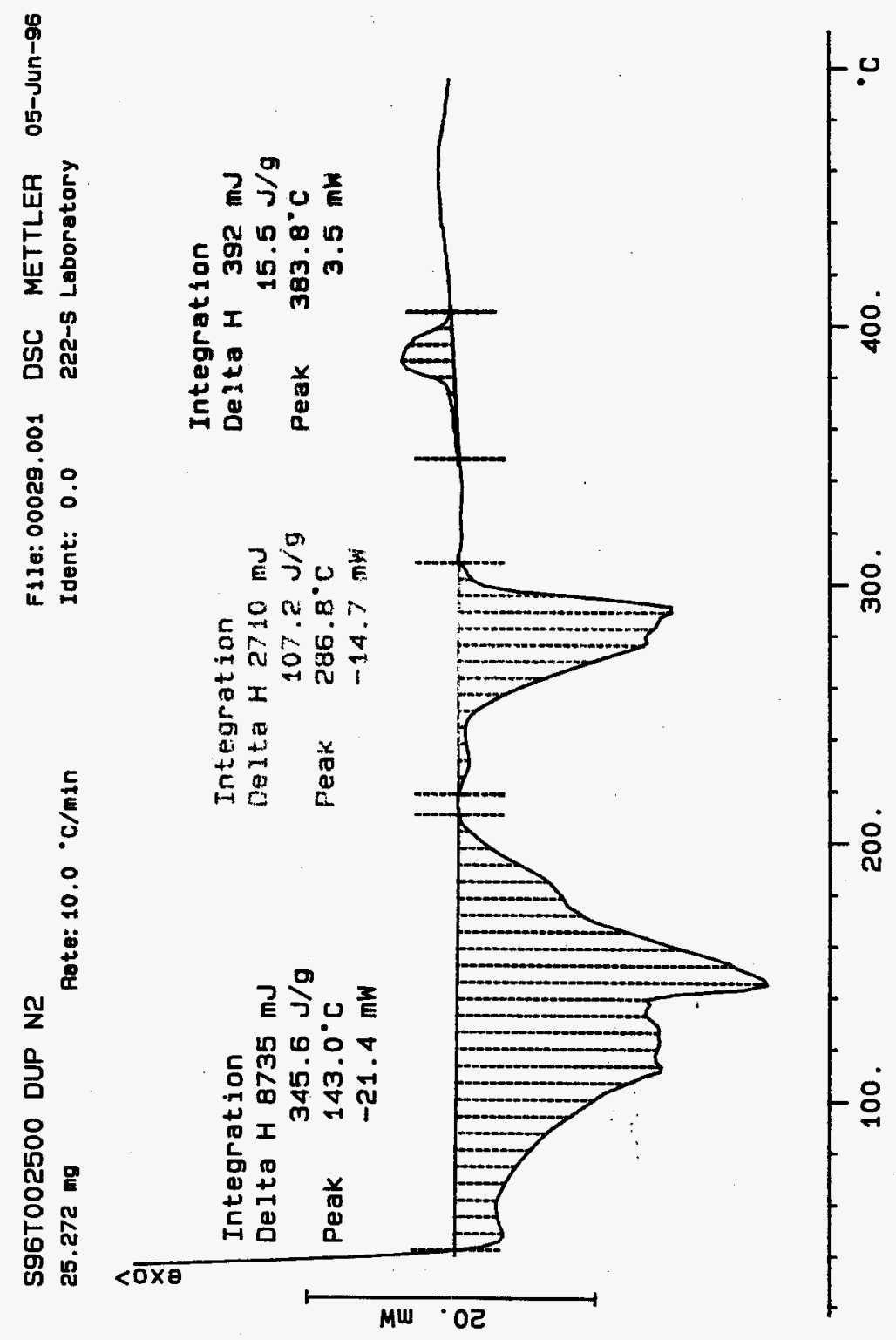


WHC-SD-WM-DP-189, REV. 0

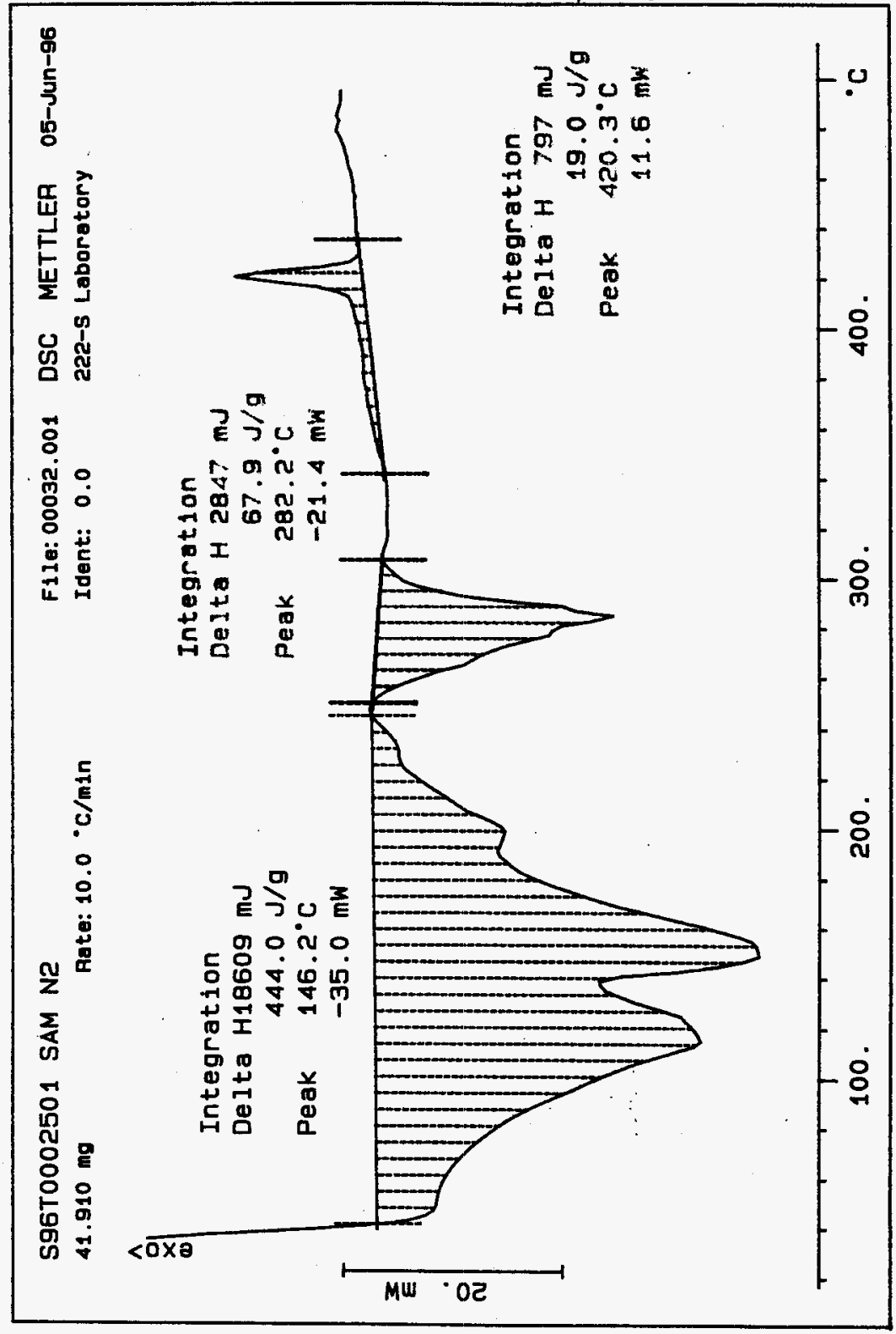


WHC-SD-WM-DP-189, REVO

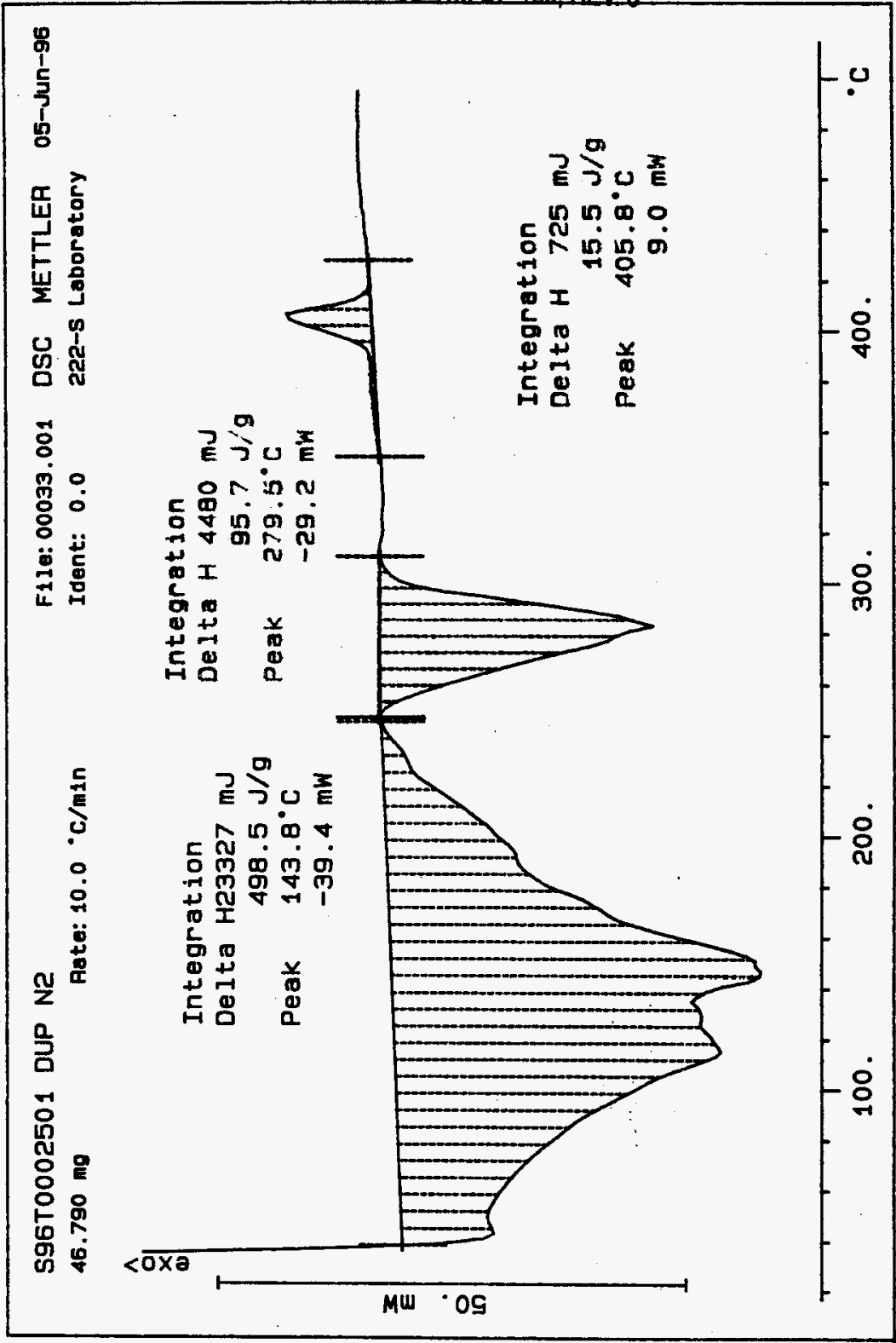




\section{LABCORE Data Entry Template for Worklist\#}

Analyst: $\quad$ CCD Instrument: DSC0 1 Book \# 12 N/4-B

Method: LA-514-113 Rev/Mod C - I

Worklist Comment: U-102 DSC, RUN UNDER N2. RCJ

\begin{tabular}{|c|c|c|c|c|c|c|c|c|}
\hline GROUP & PROJECT & $\begin{array}{l}5 \text { TYPE } \\
1 \text { STR }\end{array}$ & SAMPLE: & $\begin{array}{l}\text { R A } \cdots \text { TEST } \cdots . . . . . \\
\text { DSC-01 }\end{array}$ & $\begin{array}{l}\text { MATRIX } \\
\text { LIOUID }\end{array}$ & $\begin{array}{l}\text { ACTUAL FAND } \\
28.4531 .4^{\prime} \\
\end{array}$ & L/A & $\begin{array}{l}\text { Uwit } \\
\text { Joules/g }\end{array}$ \\
\hline 96000569 & $u-102$ & 2 SAMPLE & 596T002549 & DSC-01 & LIQUiD & 111.5 & & Joules/g \\
\hline 96000569 & $U-102$ & 3 oup - & 5961002549 & DSC-01 & LIQUID & 146.6 & ע/A & Joules/g \\
\hline 96000569 & U-102 & 4 SAMPLE & S96T002762 & DSC-01 & LIQUID & w/A & & Joules/g \\
\hline 96000569 & U-102 & 5 DUP & S96T002762 & DSC-01 & LIQUID & $b$ & $\boldsymbol{N} / \boldsymbol{A}$ & Joules/g \\
\hline
\end{tabular}

\section{Final page for worklist \# $\quad 9540$}
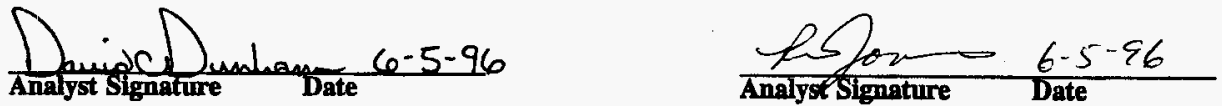

Validated by Nonasts 6.6 .96

S96100 2549 results are the sum of two exotherms.

Data Entry Comments:

Units shown for QC (SPK \& STD) may not reflect the actual wits. $D L=$ Detection Limit, $S=$ Worklist Slot Number, $\boldsymbol{R}=$ Replicate Number, $\boldsymbol{A}=$ Aliquot Code. 


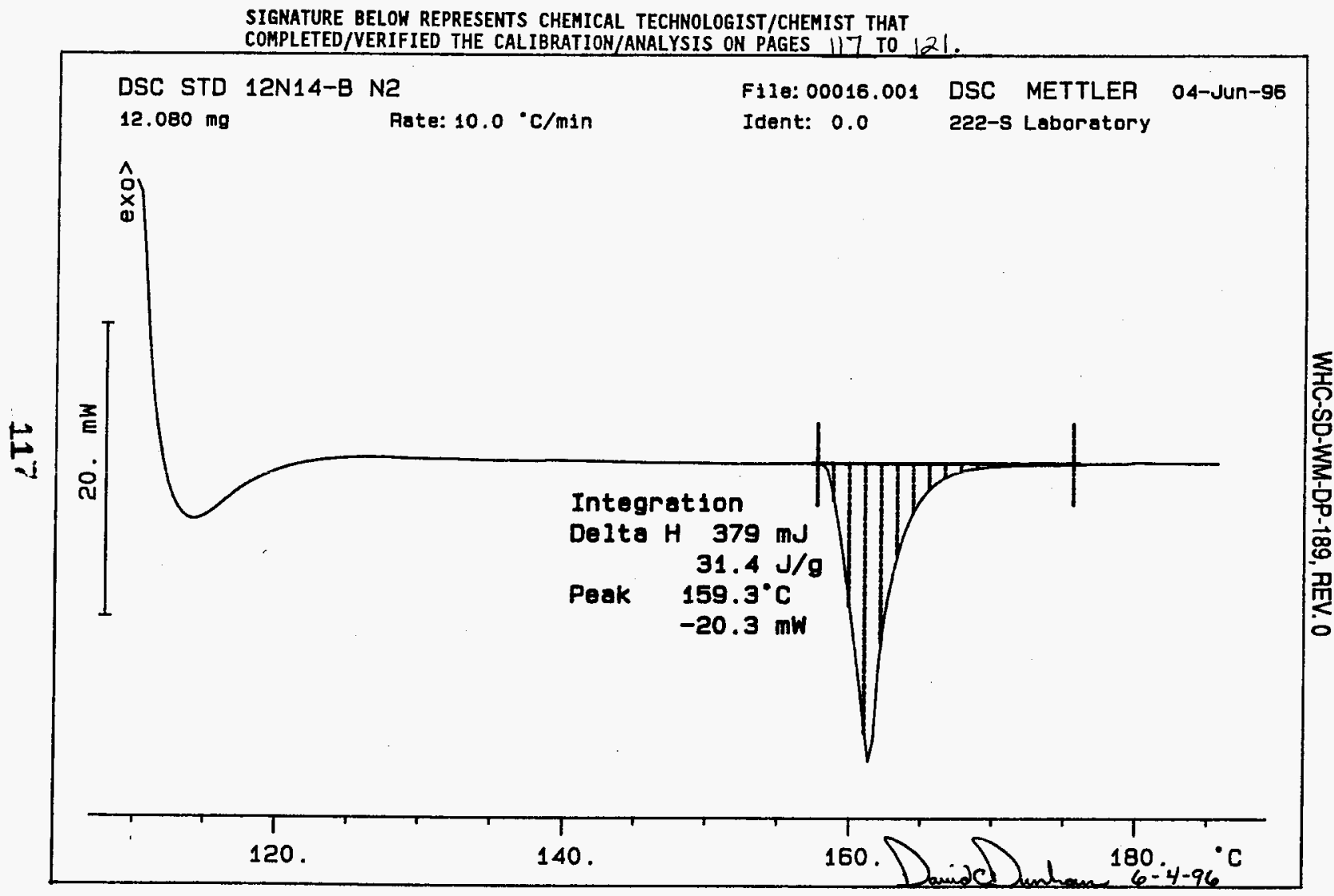




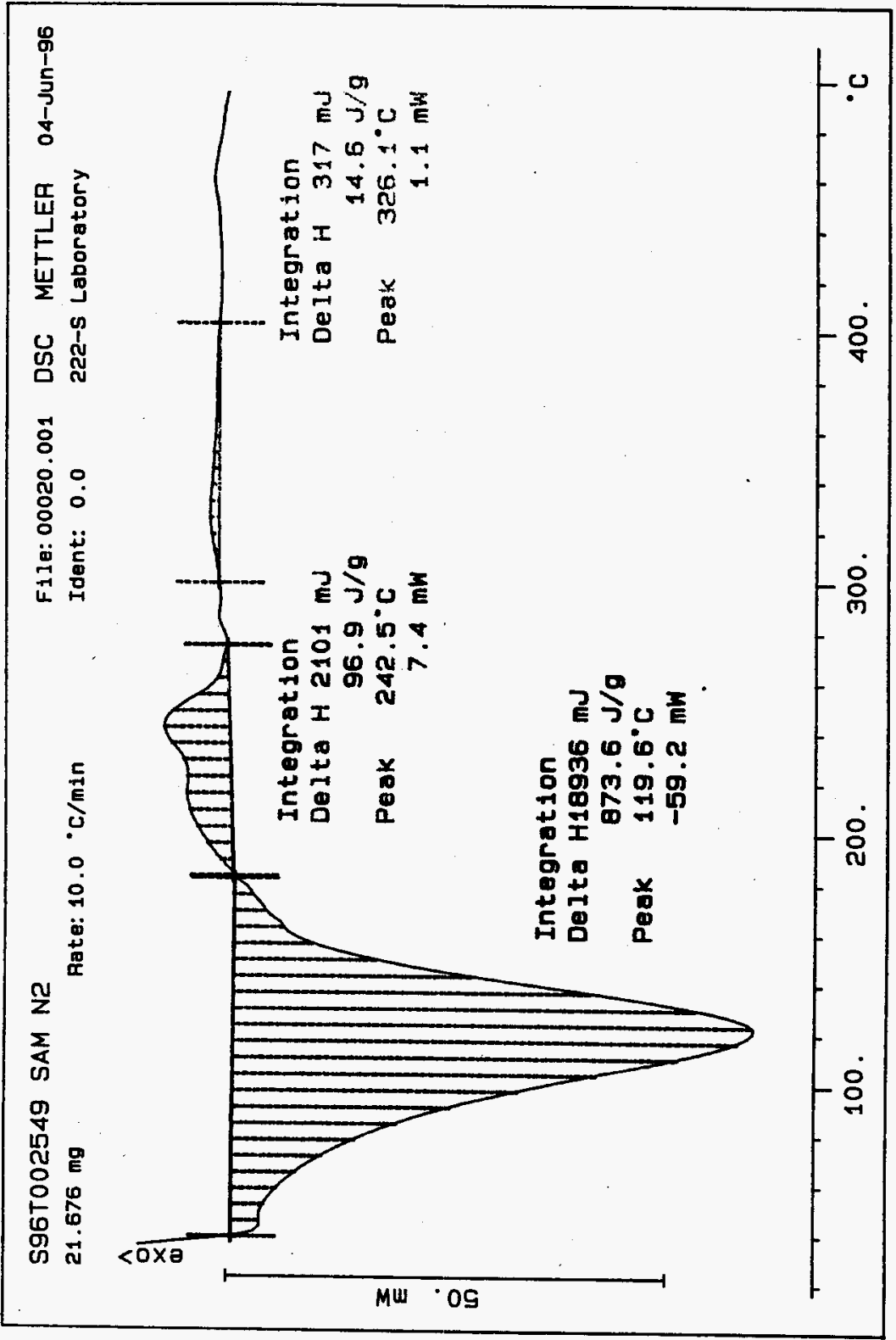


6IT

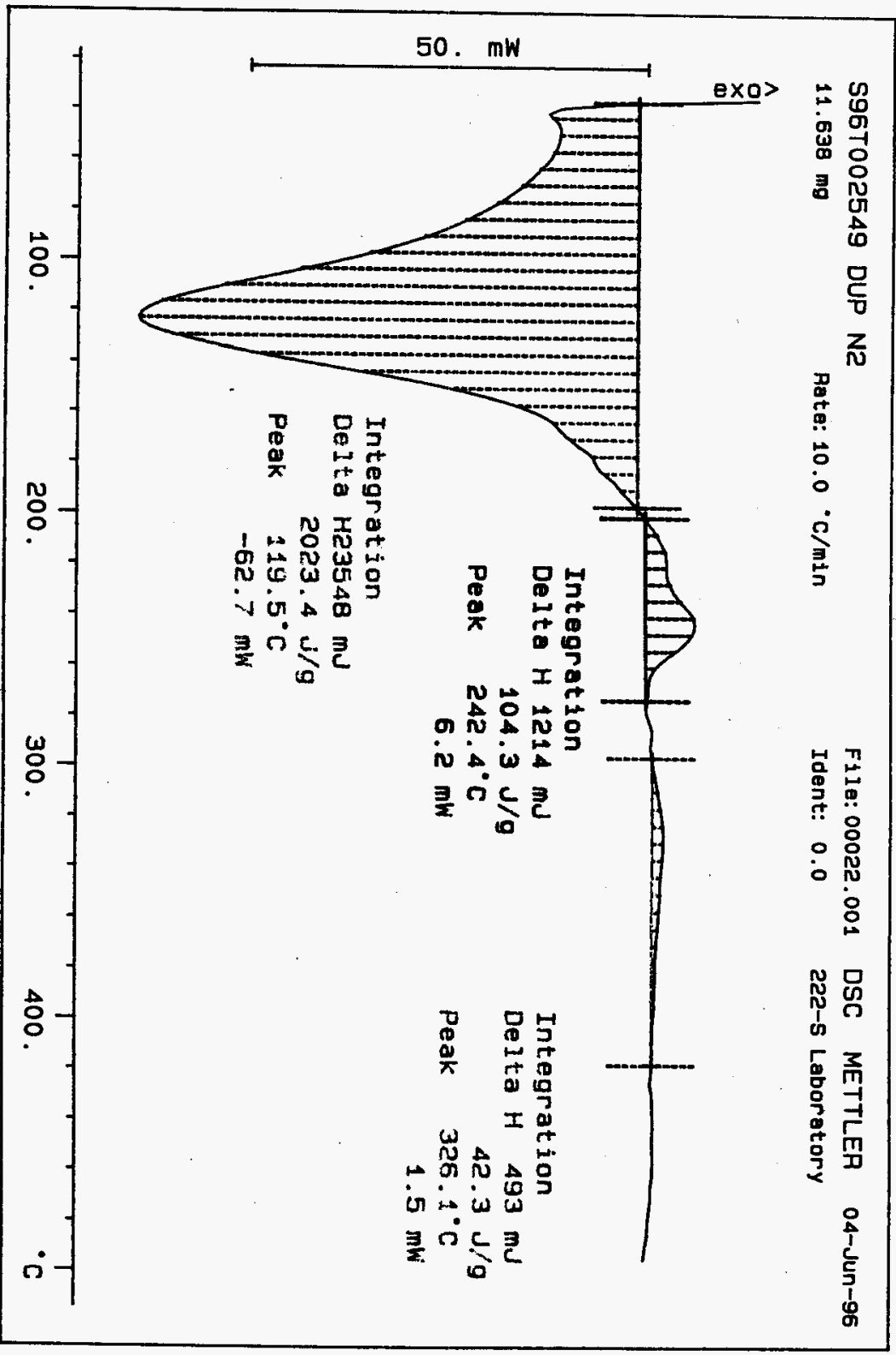


WHC-SD-WM-DP-189, REV. 0

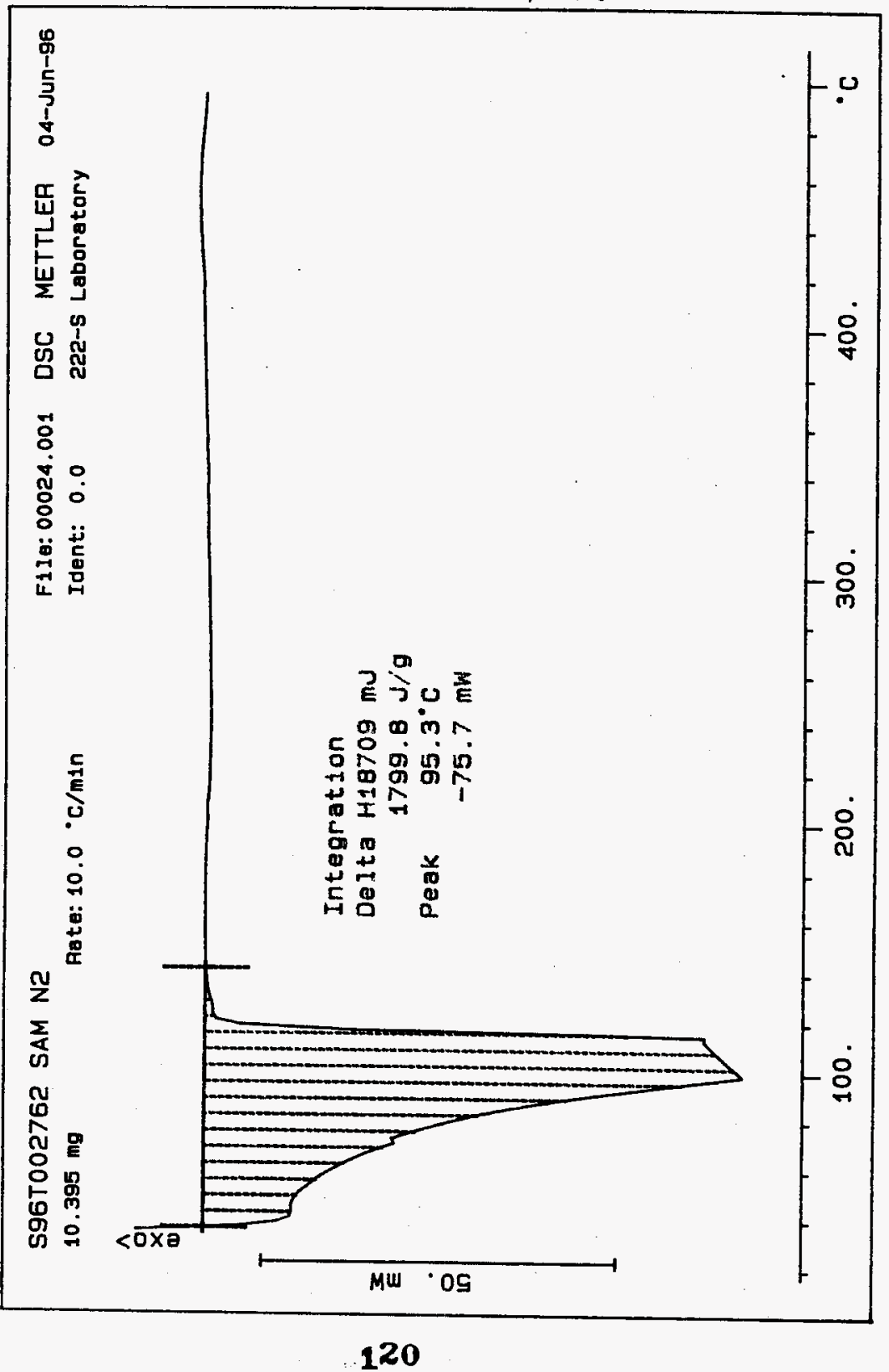


WHC-SD-WM-DP-189. REV. 0

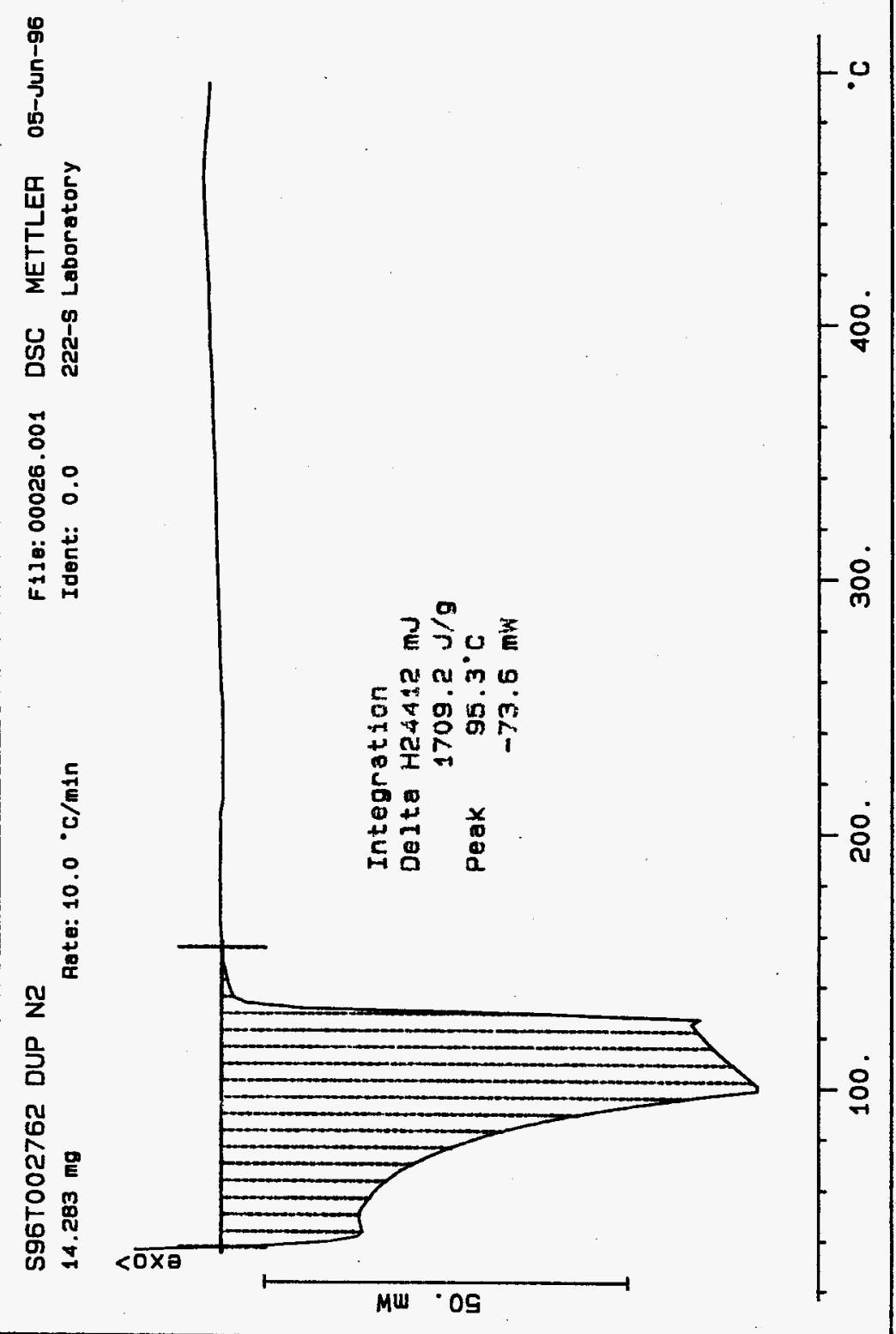

.121 
worklistrpt Version 2.1 05/15/95 WHC-SD-WM-DP-189, REV. 0

Page: 1

06/03/96 14:47

LABCORE Data Entry Template for Worklist\#

9541

Analyst: $\quad D C D \quad$ Instrument: $D S C 0 \perp$ Book \# 12 NAY

Method: LA-514-113 Rev/Mod C - 1

Worklist Comment: U-102 DSC, RUN UNDER N2. RCJ

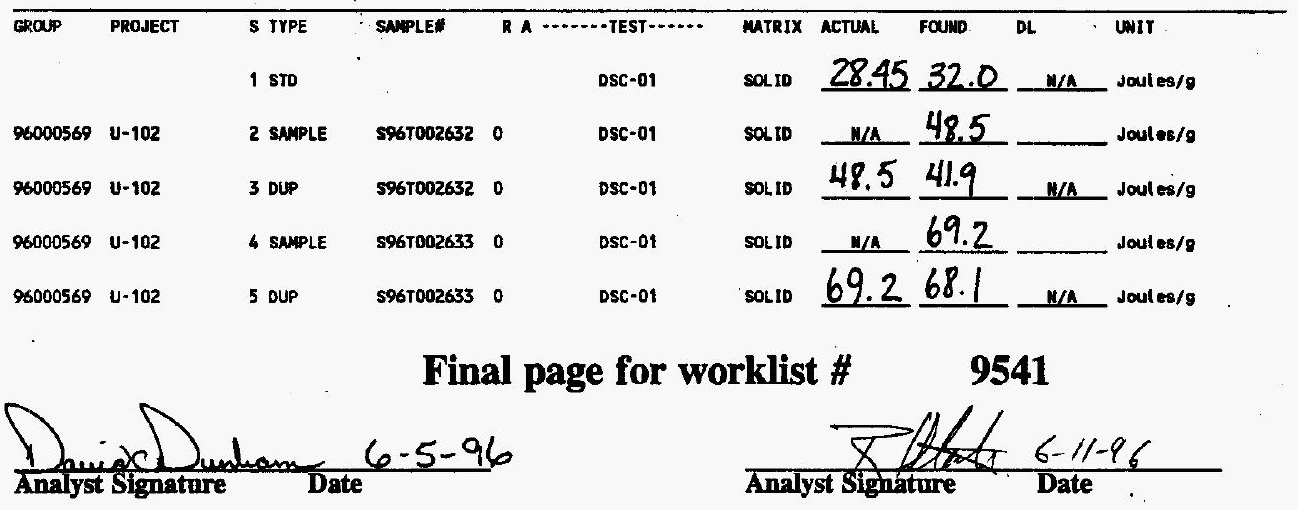

Verified/ Validated by

Blandina Valenzuela $6-12-96$

Data Entry Corneas: 9967002633 results are the sum of two exotherms.

Units shown for $Q C$ (SPK \& STD) may not reflect the actual units. $D L=$ Detection Limit, $S=$ Worklist Slot Number, $R=$ Replicate Number, $A=A$ Aliquot Code.

122 
SIGNATURE BELOH REPRESENTS CHEMICAL TECHNOLOGIST/CHEMIST THAT

COMPLETED/VERIFIED THE CALIBRATION/ANALYSIS ON PAGES 123 T0 127

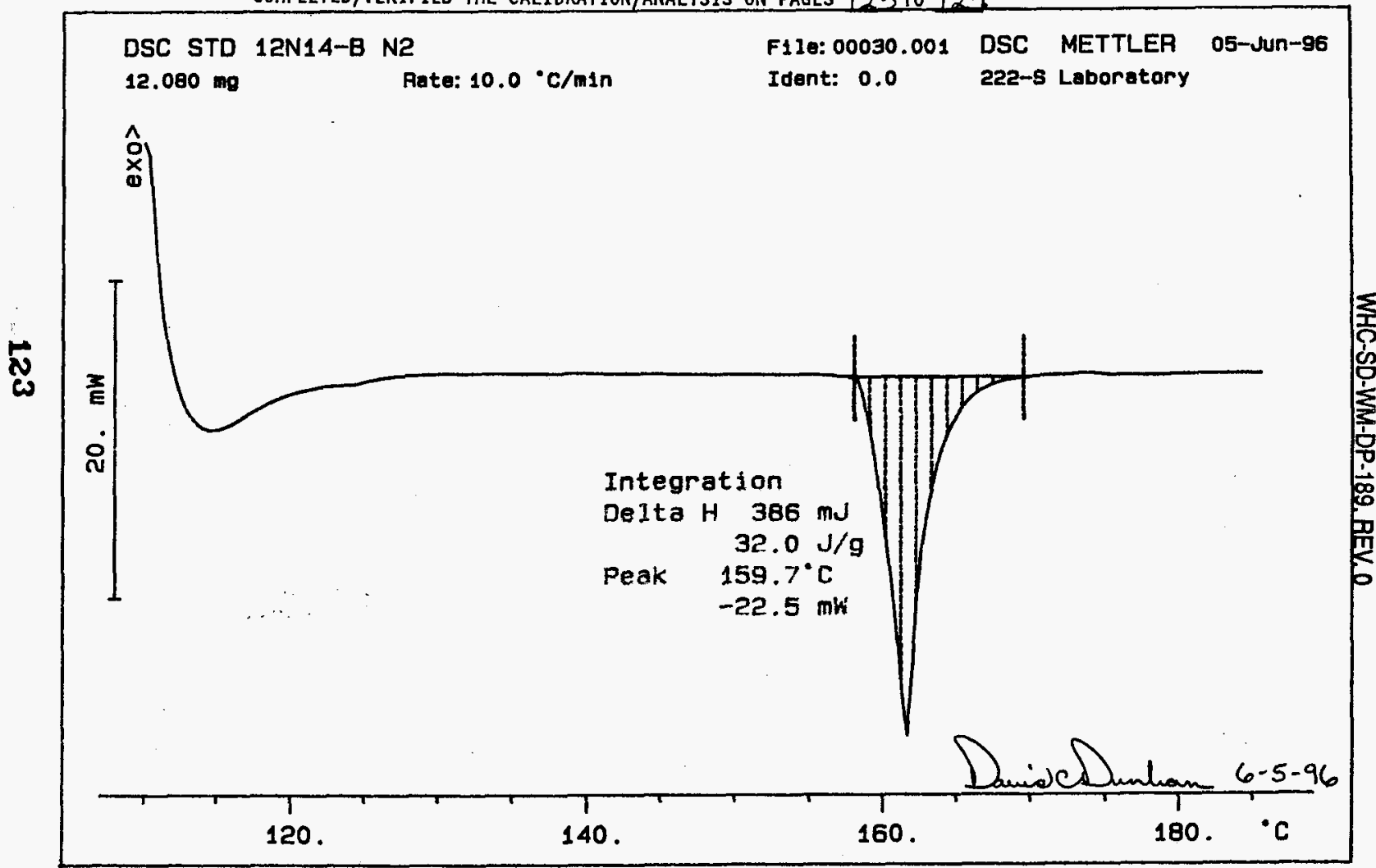


WHC-SD-WM-DP-189, REV. 0

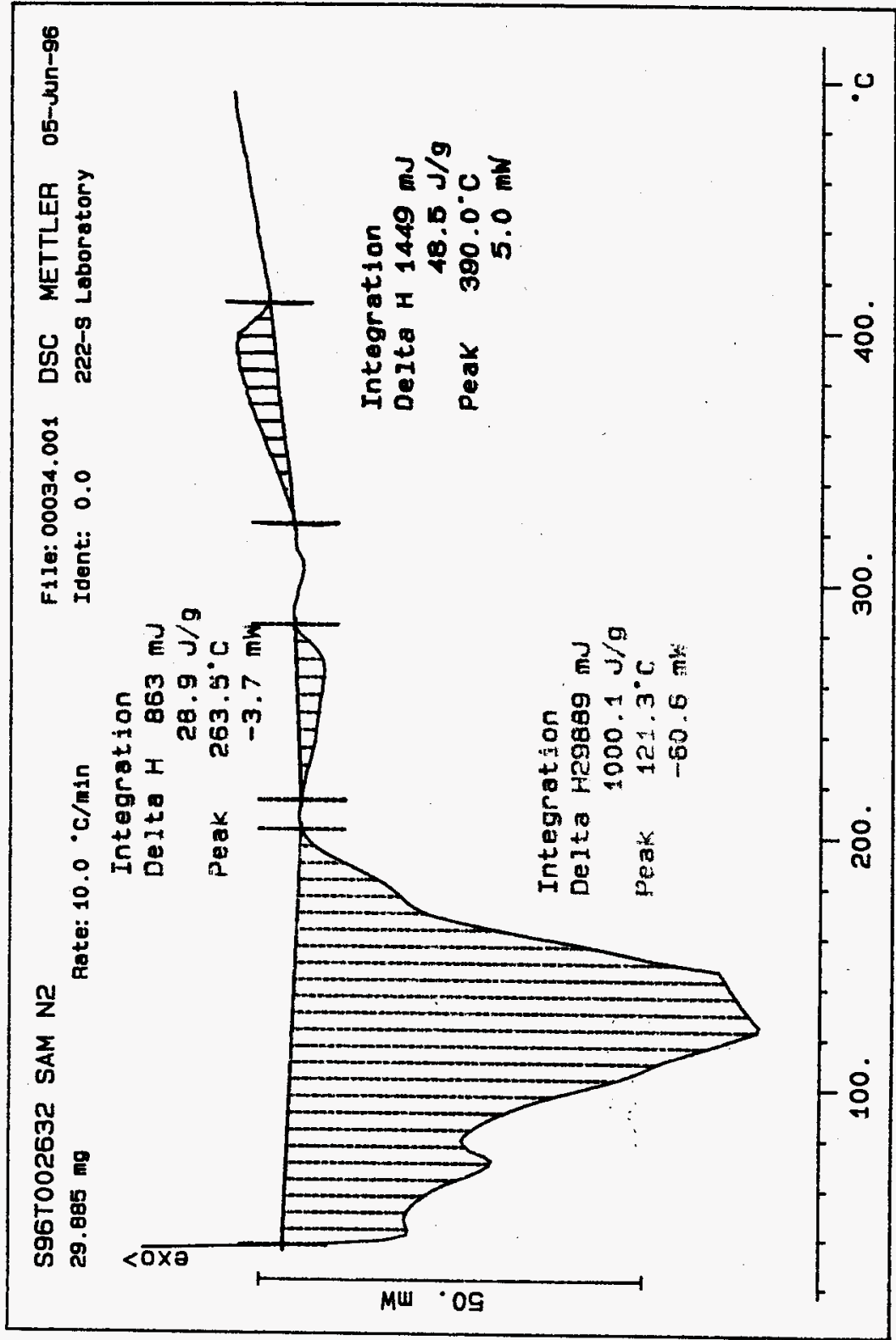




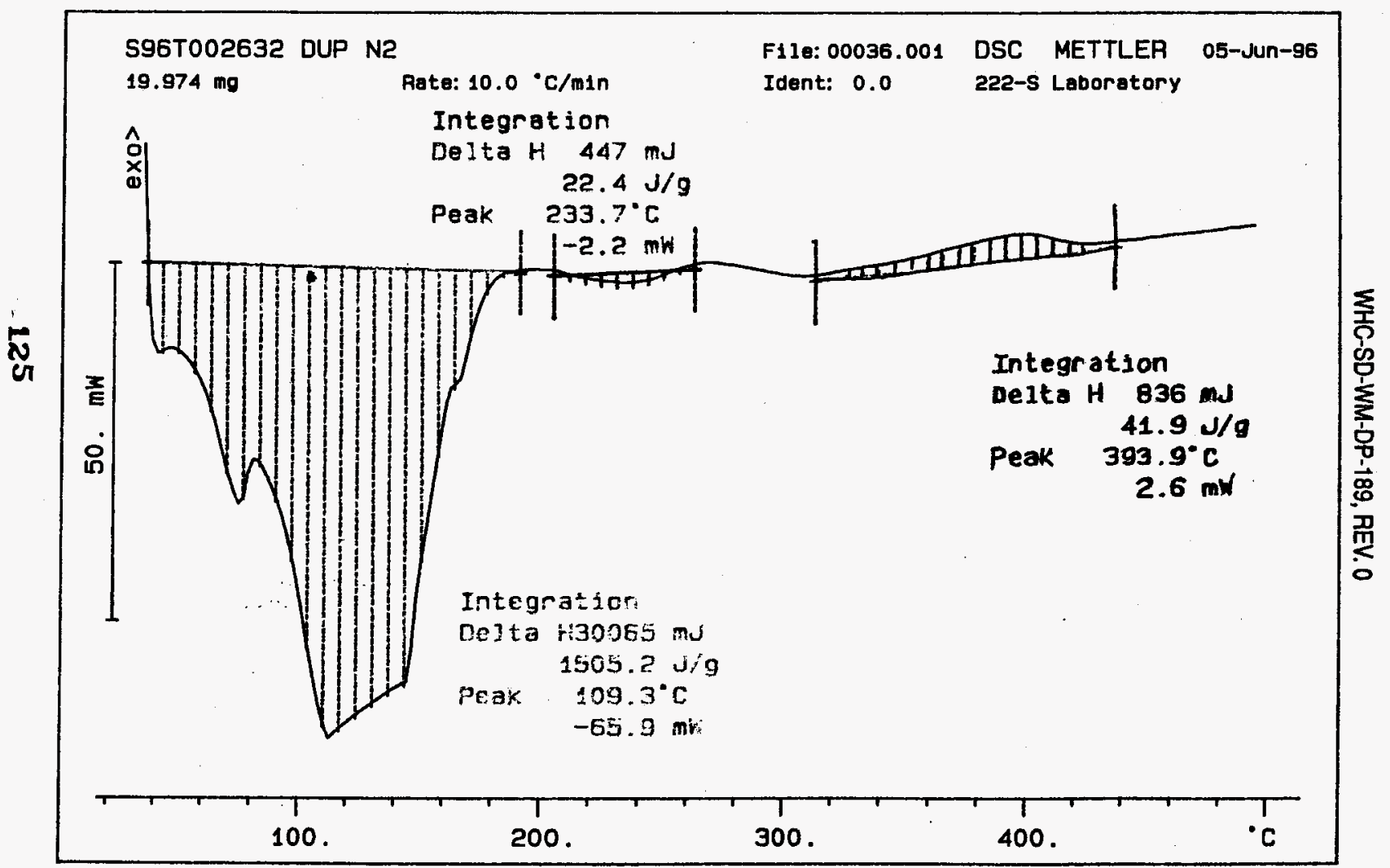




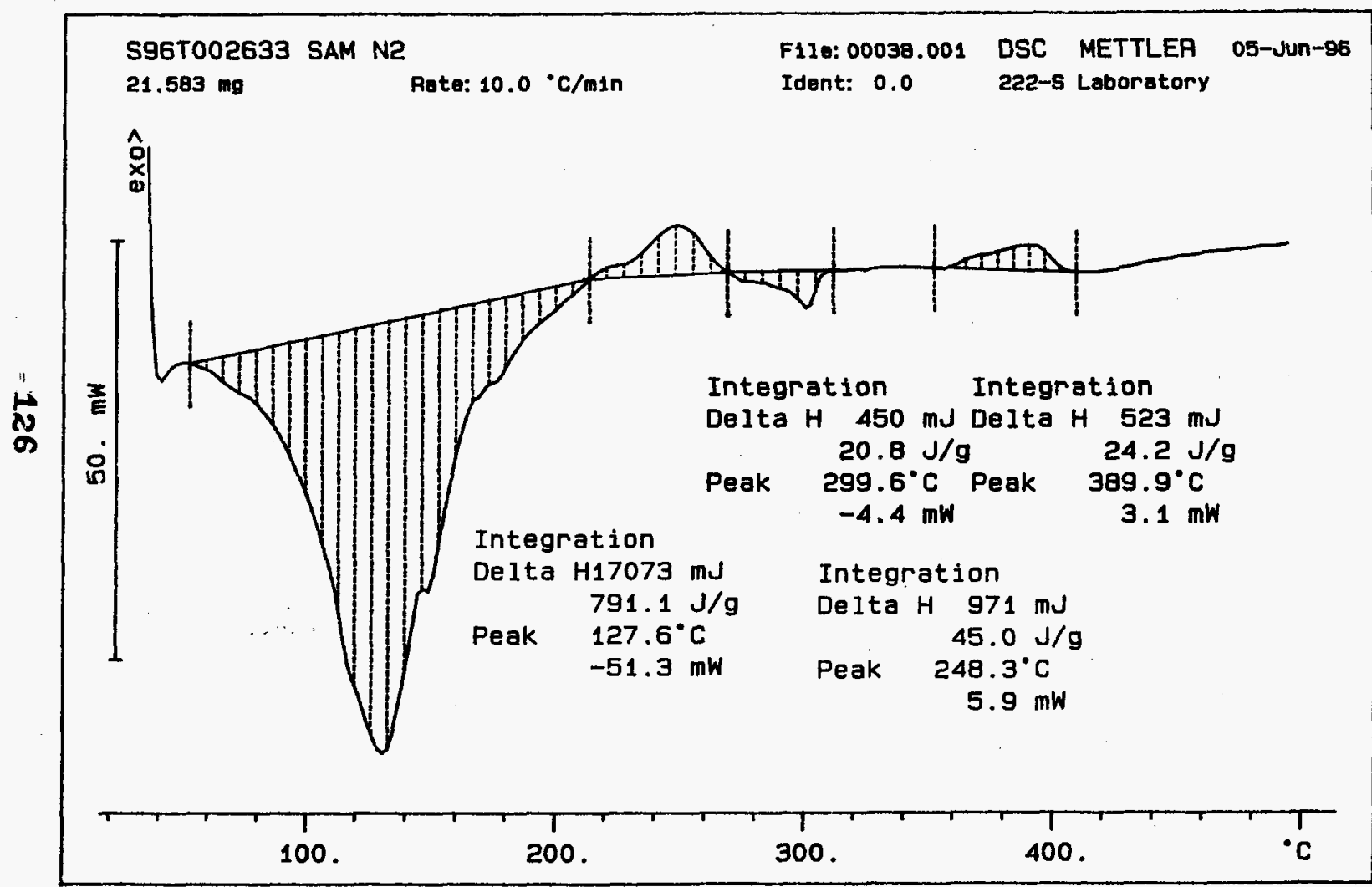




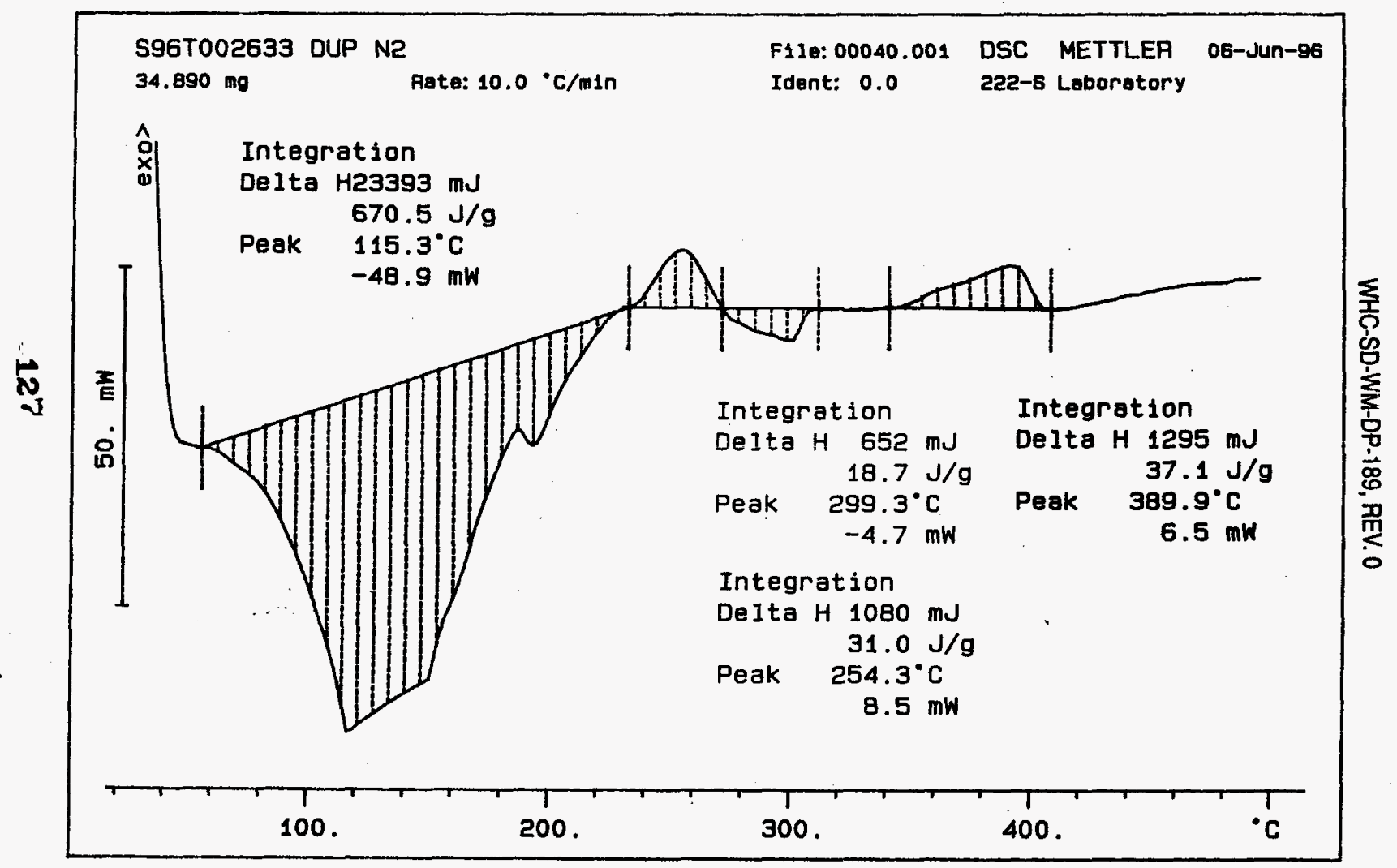


WHC-SD-WM-DP-189, REV. 0

worklistrpt Version 2.1 05/15/95

Page: 1 $06 / 11196$ 14:11

LABCORE Data Entry Template for Worklist\#

9543

Analyst: $\quad$ LCD $\quad$ Instrument: SC 3 Book \# 12N14 B

Method: LA-514-114 Rev/Mod C - I

Worklist Comment: U-102 DSC, RUN UNDER N2. RCJ

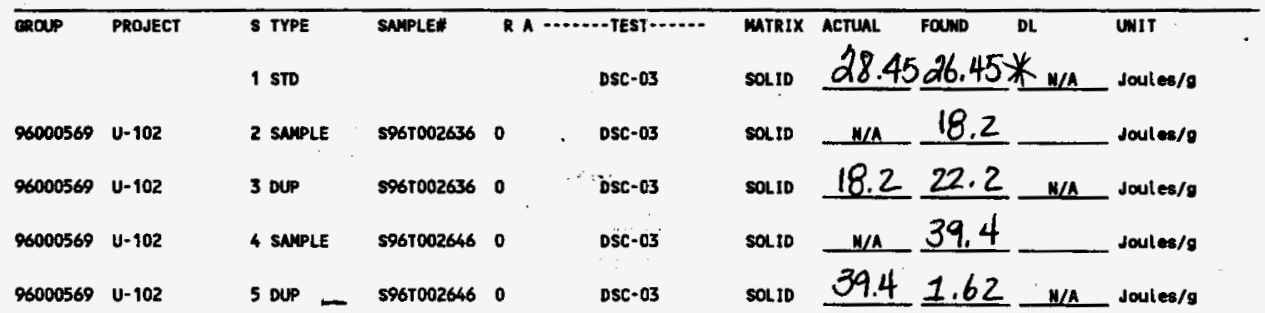

Final page for worklist \# $\quad 9543$

See attached for signatures

Analyst Signature Date ${ }_{6} / 1 / 96$

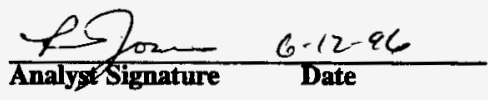

Verified/Valideded by

Binding Valenizfila 613196

Data Entry comments: 3967002646 did not have a triplicate because it was not close to the notification limit.

Units shown for $Q C$ (SPK \& STD) may not reflect the actual units. $D L=$ Detection Limit, $S=$ Worklist Slot Number, $R=$ Replicate Number, $A=$ Aliquot Code.

$$
=128
$$




\section{LABCORE Data Entry Template for Worklist\#}

\section{Analyst: $\quad$ DCD Instrument: DSCO Book \# $\angle 2 N 44 B$}

Method: LA-514-113 Rev/Mod

Workhist Comment: U-102 DSC, RUN UNDER N2. RCJ

\begin{tabular}{|c|c|c|c|c|c|c|c|c|c|c|}
\hline \multirow[t]{2}{*}{ ERoup } & \multirow[t]{2}{*}{ PROJECT } & S TYPE & \multirow[t]{2}{*}{ SAYPLE\# } & \multicolumn{2}{|c|}{ 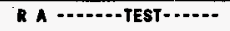 } & \multirow{2}{*}{$\begin{array}{l}\text { MtRIX } \\
\text { SOLID }\end{array}$} & \multirow[t]{2}{*}{ ACTUAL } & \multirow[t]{2}{*}{ FOUND } & \multirow[t]{2}{*}{$\mathbf{D L}$} & \multirow{2}{*}{$\begin{array}{l}\text { UAlT } \\
\text { Joules/g }\end{array}$} \\
\hline & & 1 STD & & & DSE-01 & & & & & \\
\hline 96000569 & $U-102$ & 2 SAMPLE & S96t002636 & 0 & DSC-01 & 50010 & $\boldsymbol{N} / \boldsymbol{A}$ & & & Joules/g \\
\hline 96000569 & $U-102$ & 3 DuP & $\$ 961002636$ & 0 & DSC-01 & SOL10 & & & $y / A$ & Joutes/o \\
\hline 96000569 & $U-102$ & 5 DUP & 5961002646 & $\mathbf{0}$ & Dsc-01 & SOLID & & & $N / A$ & Joules/s \\
\hline
\end{tabular}

\section{Final page for worklist \# 9543}

Dinc) Duter- 6-6-96

Analyst Signature Date

$$
\begin{aligned}
& \text { DSC-03 instrument } \\
& \text { was used. } 611196
\end{aligned}
$$


Curve 1: DSC

F11e info: IND060601 Thu un 6 20: 22: 381996

Somple Neight: 10.650 ag

12N14-B INDIUM AT 10CNIN

SIGNATURE BELOW REPRESENTS CHEMICAL TECHNOLOGIST/CHEMIST THAT

COMPLETED/VERIFIED THE CALIBRATION/ANALYSIS ON PAGES 130 TO 134.

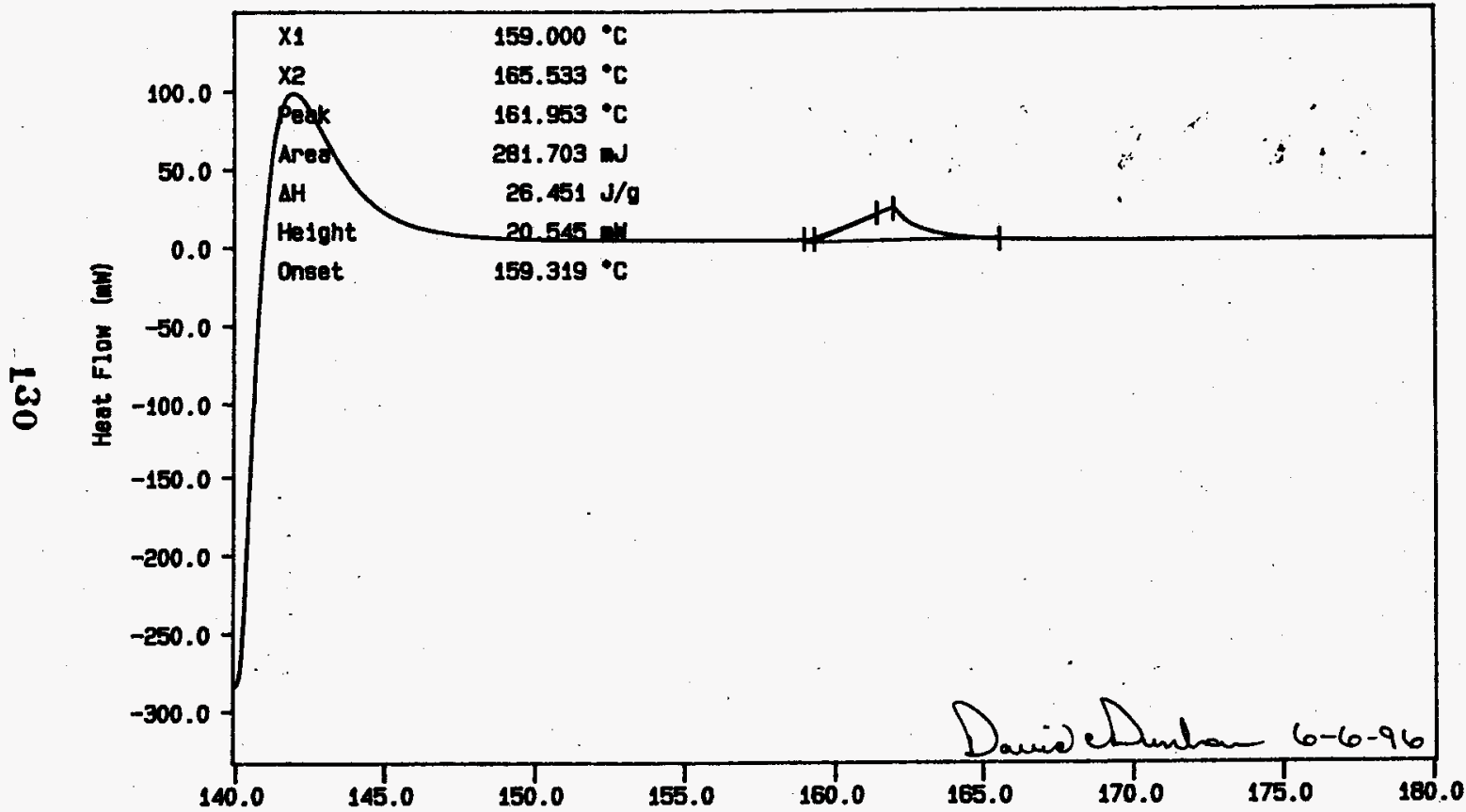

Ne, EXOTHERM DONN

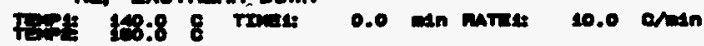

Temperature ( $\left.{ }^{\circ} \mathrm{C}\right)$

DC Dunhen

PERKIN-ELIEA

7 Ser1es Therad Analysis Systei Thu Jun 6 20:55 17. 1990 
Curve 1: DSC

F1le info: SAM060603 Thu un 6 18:49:35 1996

Sample Waight: 20.570

s96T0022636 SAM

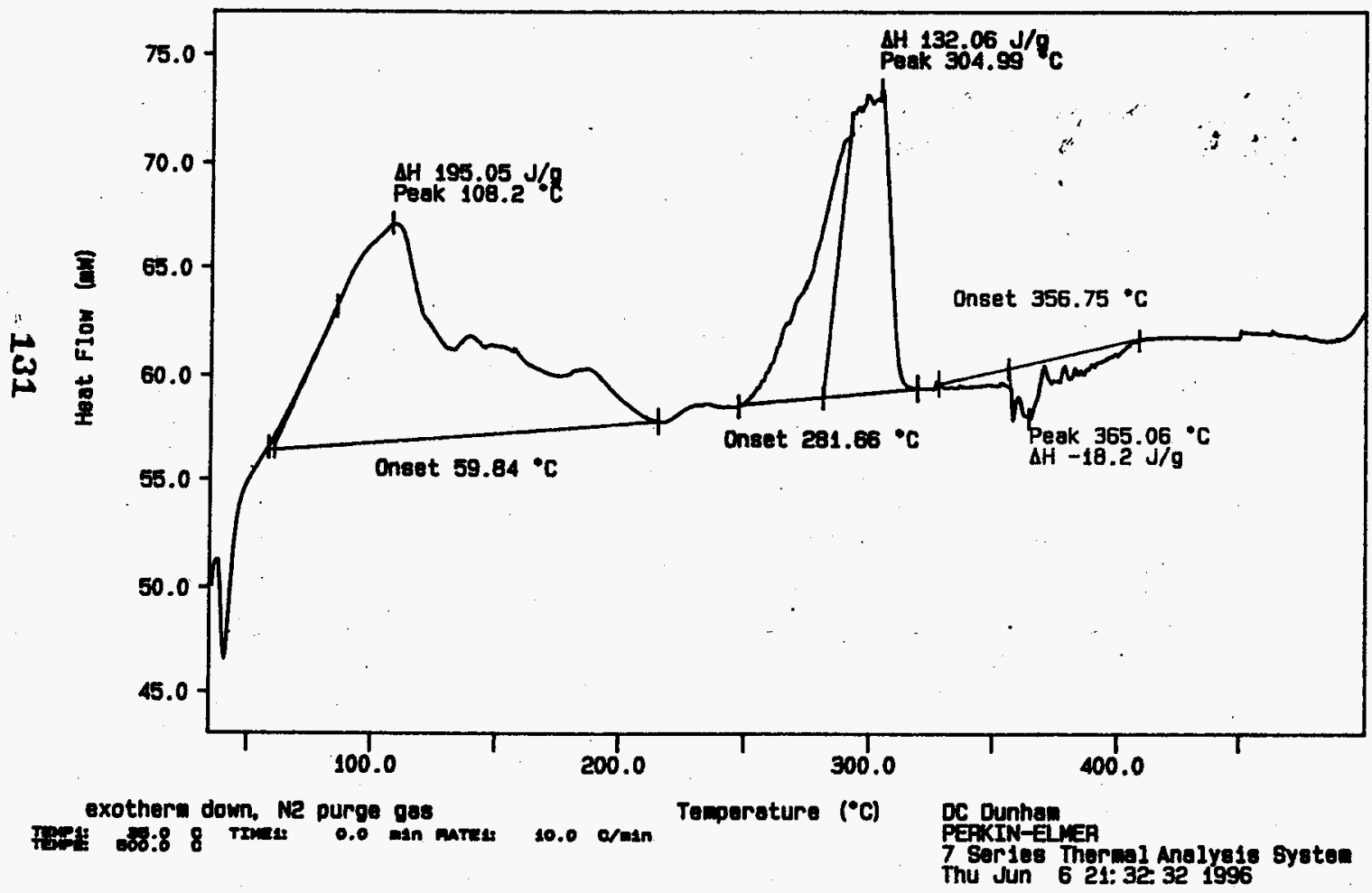




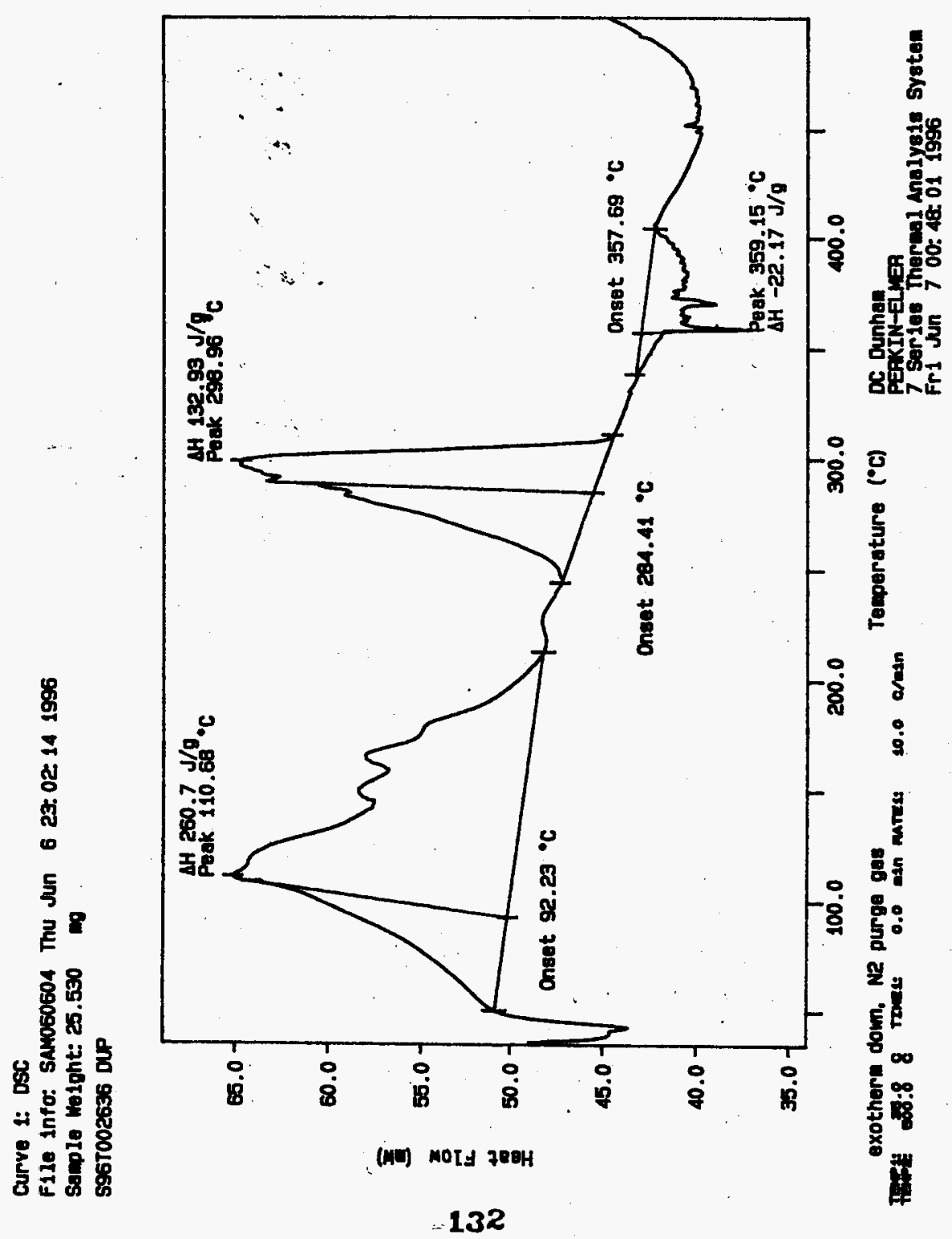


Curve 1: DSC

F1le info: SAmo60605 Fr1 un 7 01:21: 261996

Sanple Wejght: 8.520

596T002646 SAM

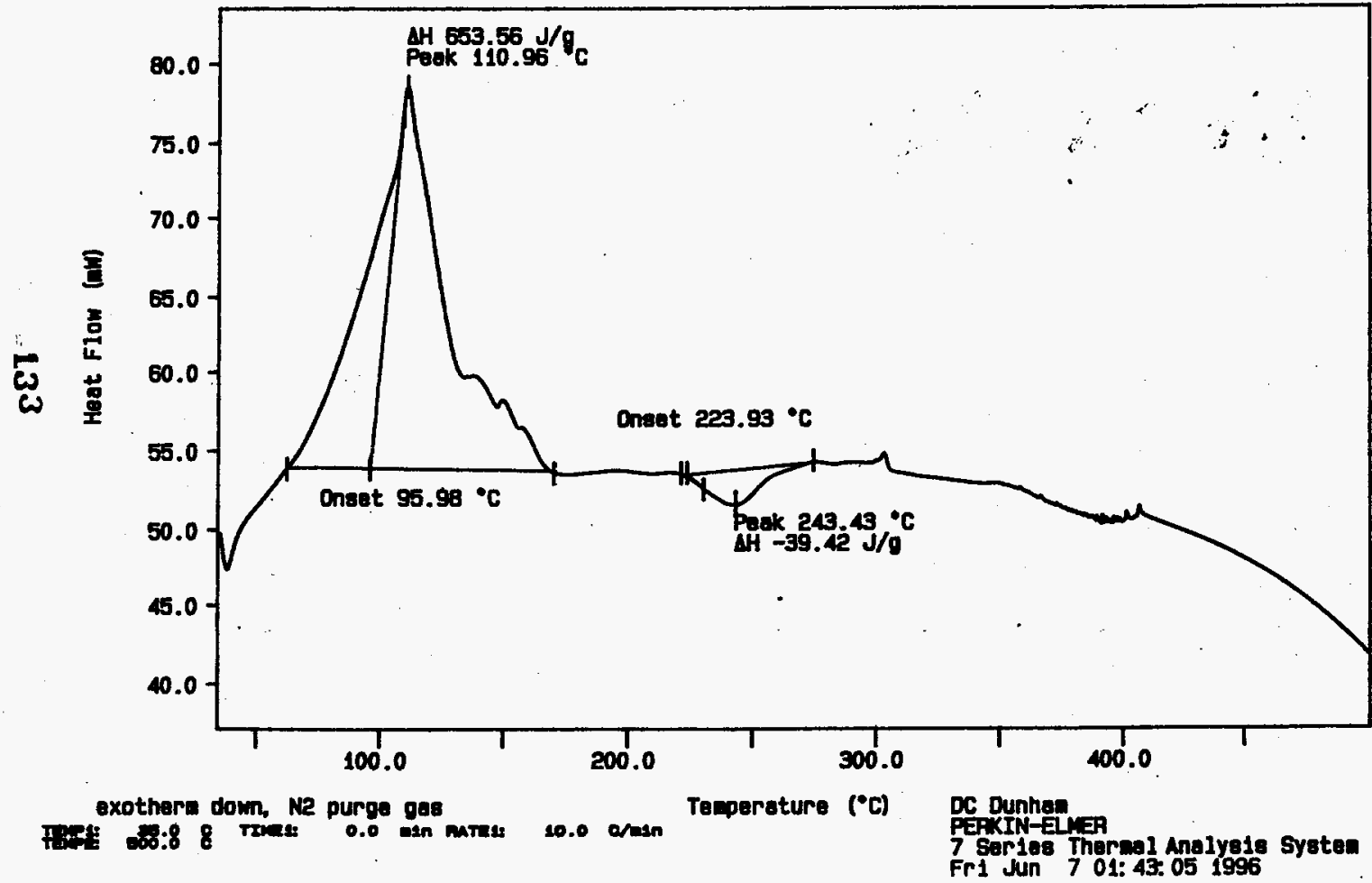


Curve 1: DSC

F1le info: SAM060606 Fri un 7 03:51: 171996

Sanple We1ght: 27.240

5967002646 DUP

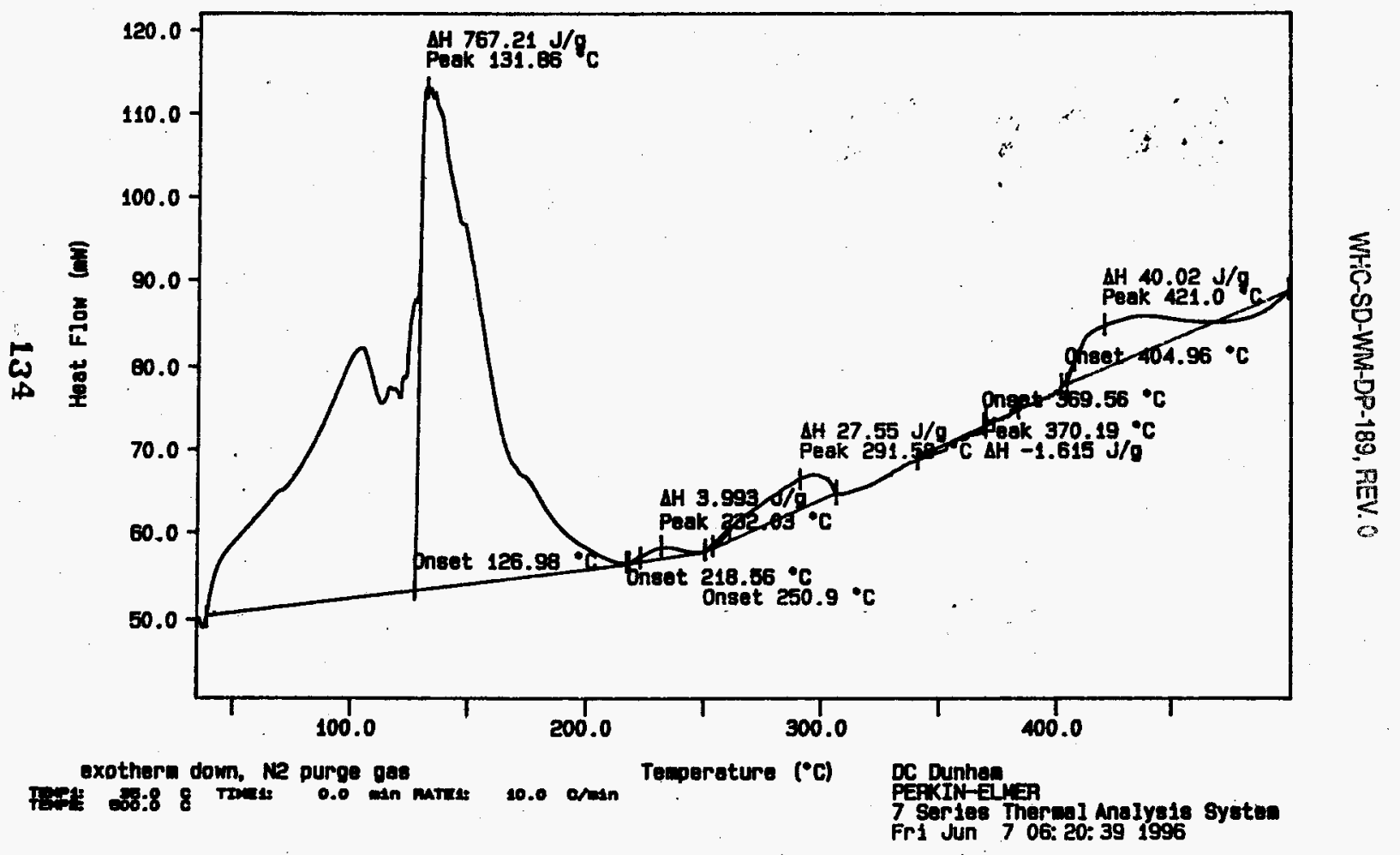




\section{LABCORE Data Entry Template for Worklist\#}

Analyst: $D C D$ Instrument: $D S C O \perp$ Book \# 12 N14 B

Method: LA-514-113 Rev/Mod C -

Worklist Comment: U-102 DSC, RUN UNDER N2. RCJ

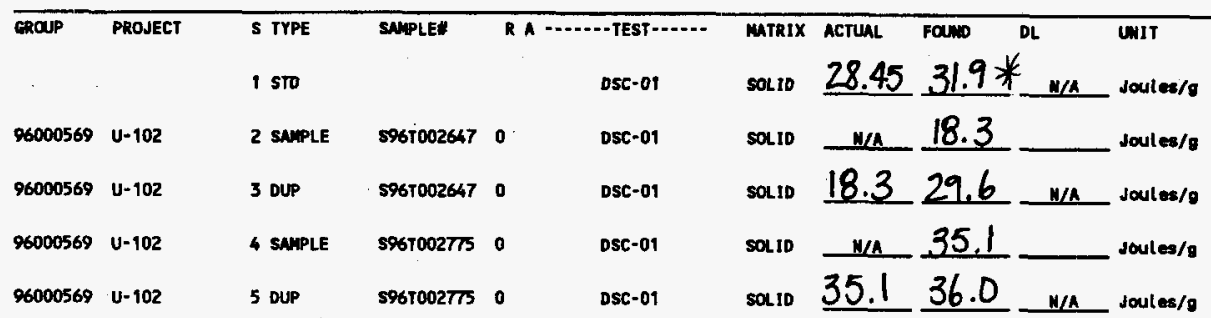

\section{Final page for worklist \# $\quad 9544$}
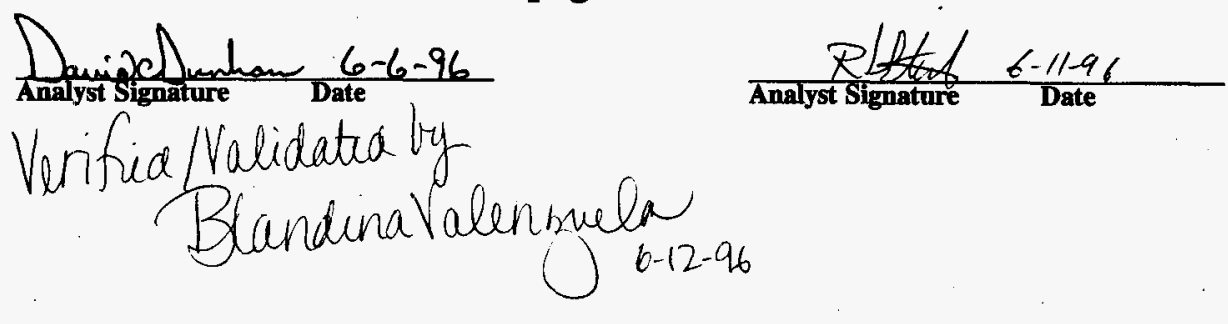

596700 2647 produced an endotherm at approximately $295.6 \mathrm{C}$ S96700 2647 produced an endotherm at app
this peak is probably indicatice of a luminum Hydroxide decomposition
reaction.

Data Entry Comments:

Units shown for QC (SPK \& STD) may not reflect the actual wnits. $D L=$ Detection Limit, $S=$ Worklist Slot Number, $\boldsymbol{R}=$ Replicate Number, $A=A$ Aliquot Code. 


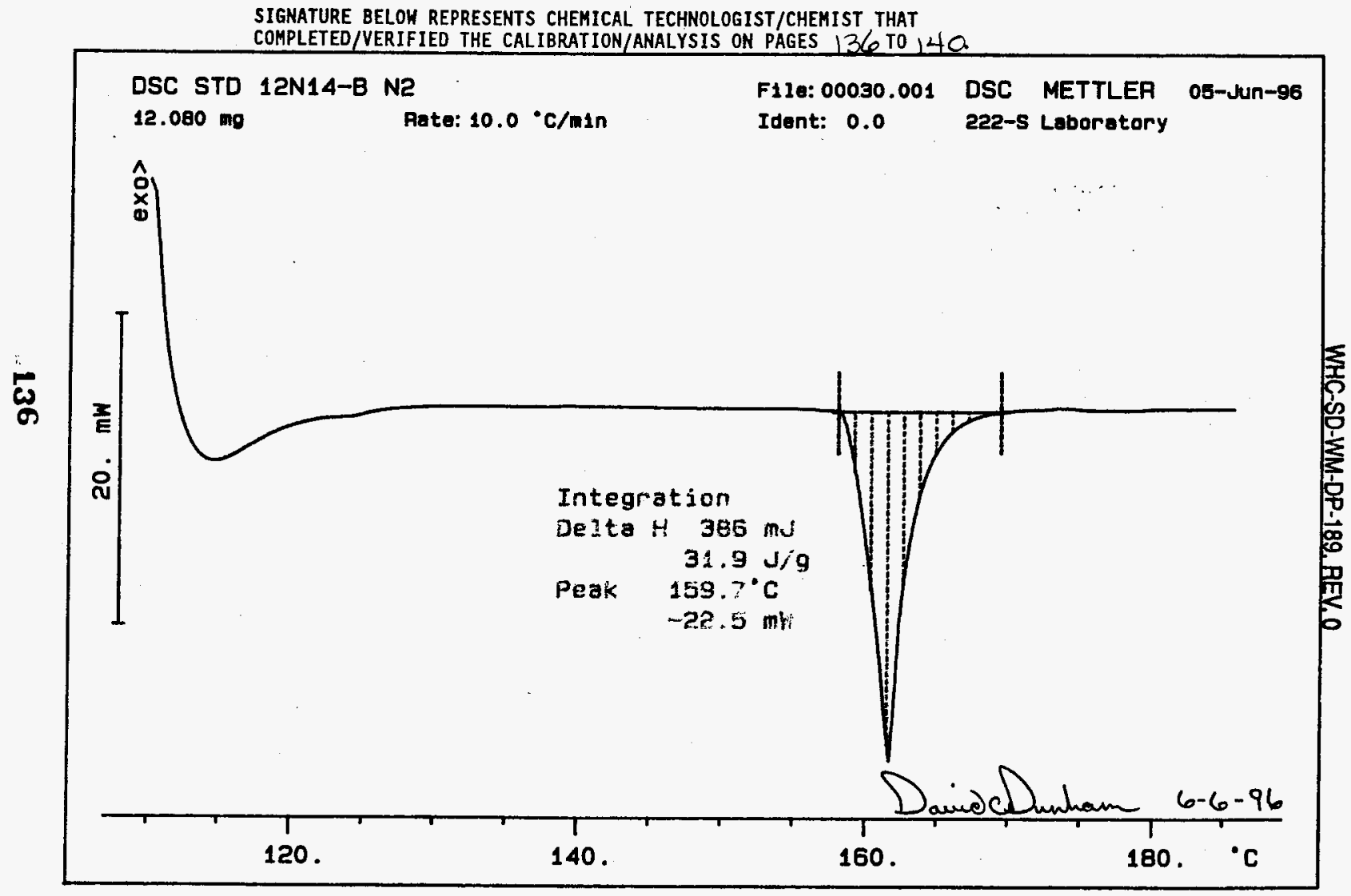




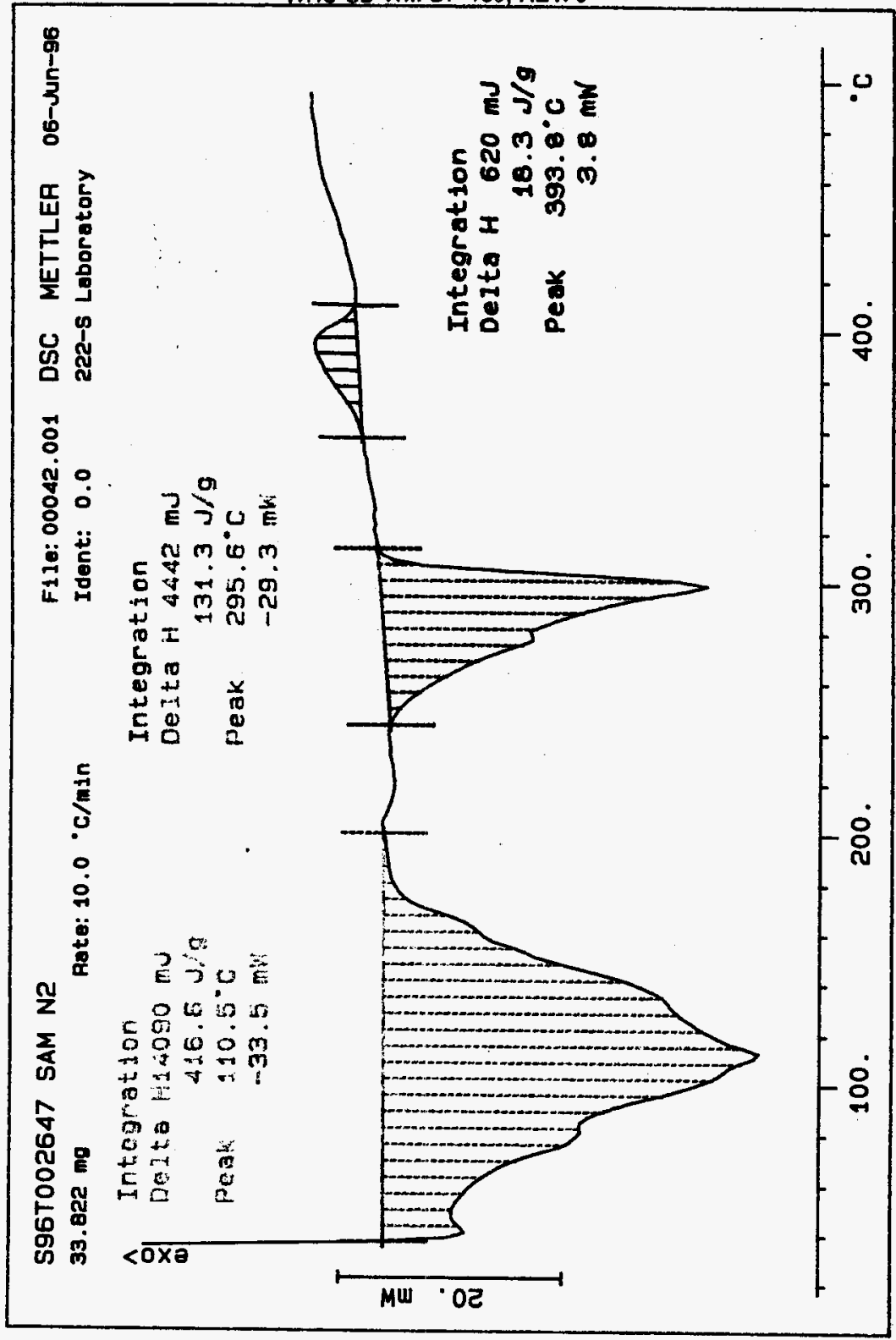




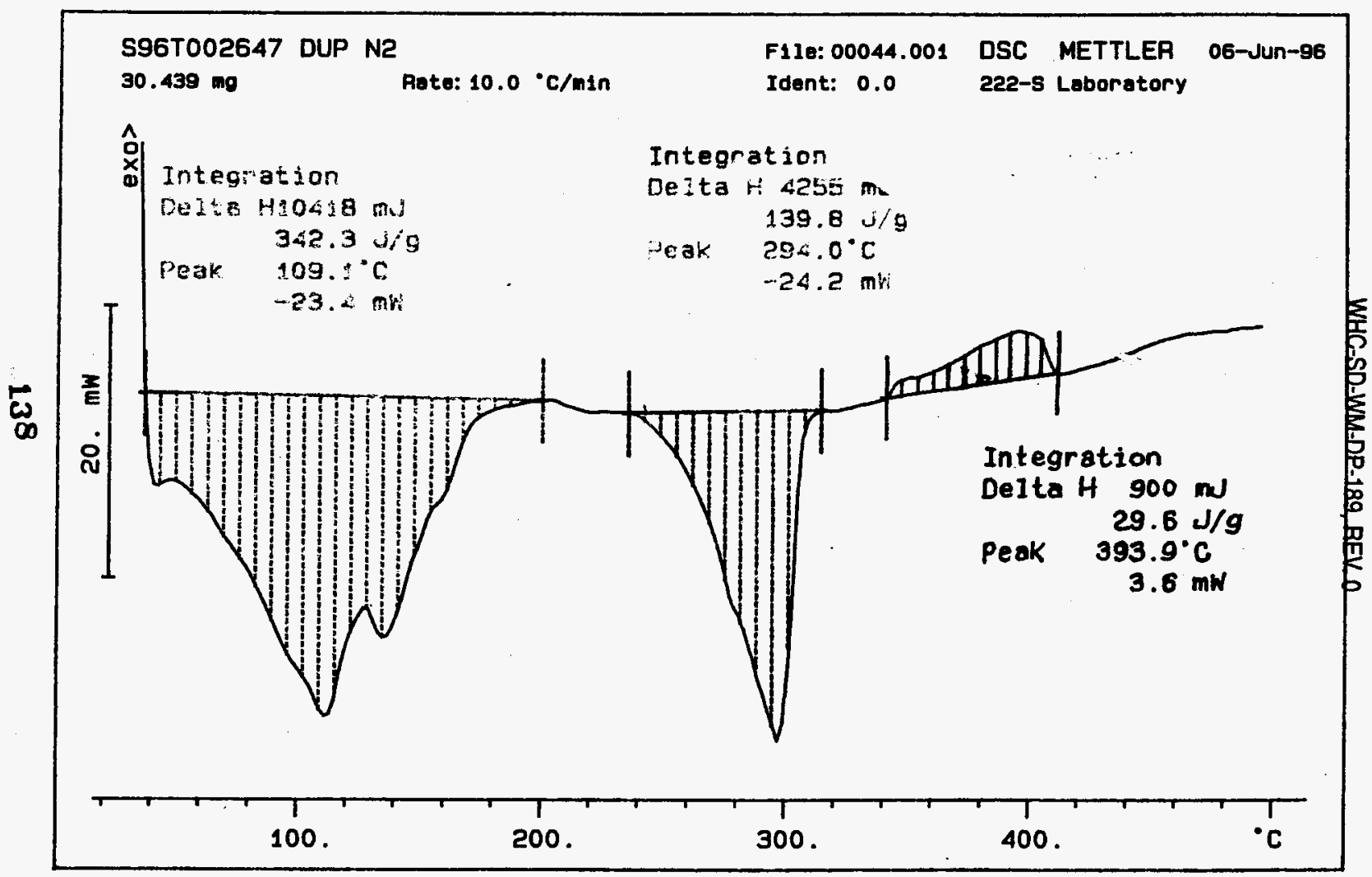




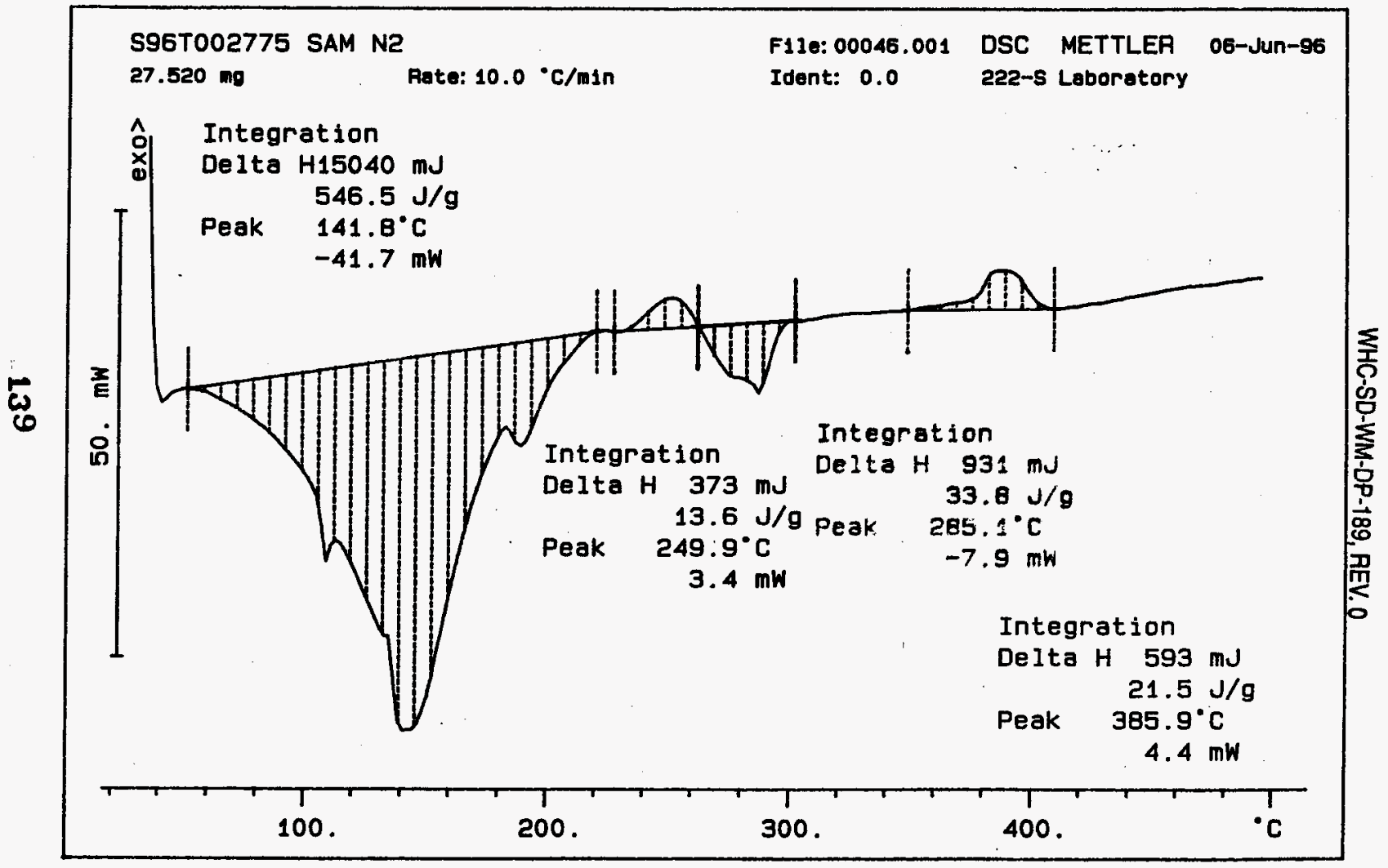




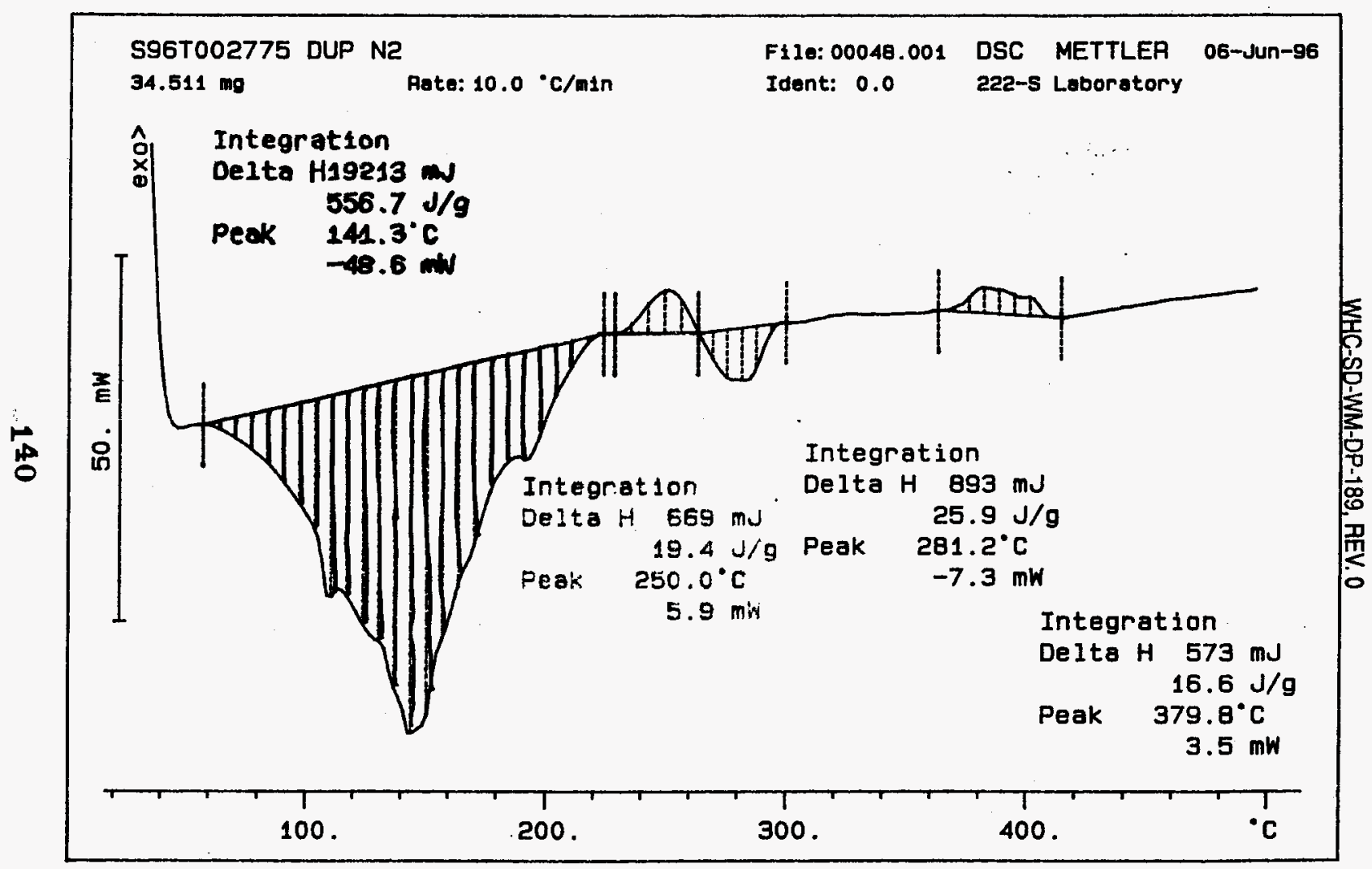


worklistrpt Version 2.1 05/15/95

WHC-SD-WM-DP-189, REV. 0

06/03/96 14:50

LABCORE Data Entry Template for Worklist\#

Page: $I$

9545

Analyst: $D C D$ Instrument: DSCO I Book $12 N / 4 B$

Method: LA-514-113 Rev/Mod C-1

Worklist Comment: U-102 DȘC, RUN UNDER N2. RCJ

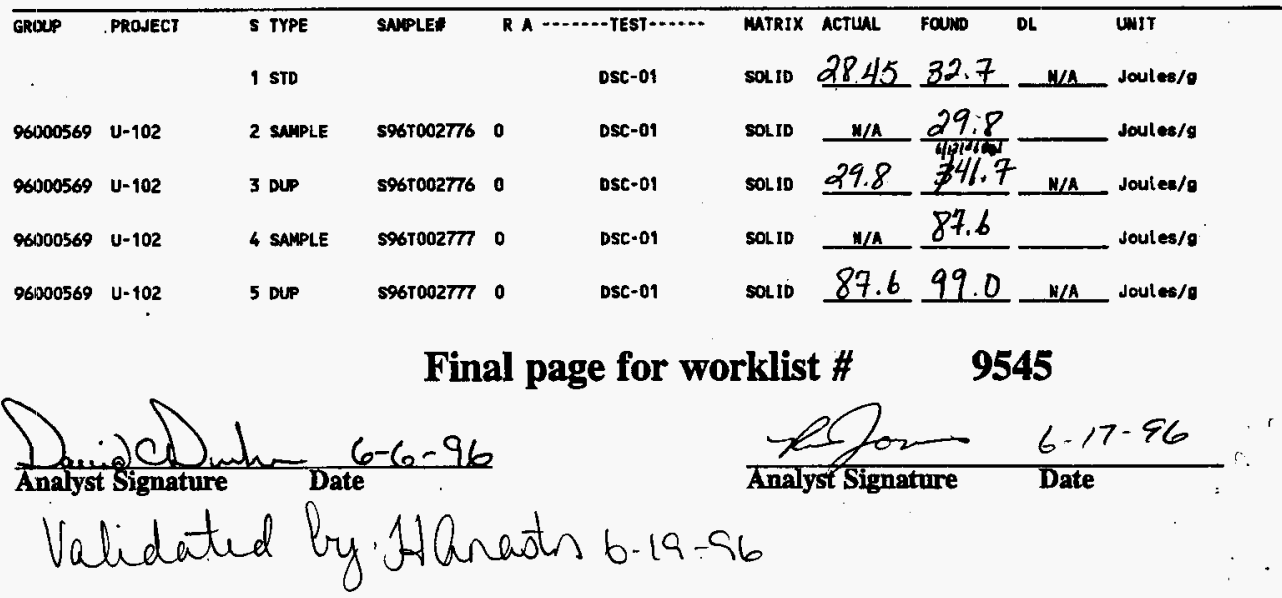

Data Entry Comments:

Units shown for QC (SPK \& STD) may not reflect the actual units. $D L=$ Detection Limit, $S=$ Worklist Slot Number, $R=$ Replicate Number, $A=$ Aliquot Code.

141 


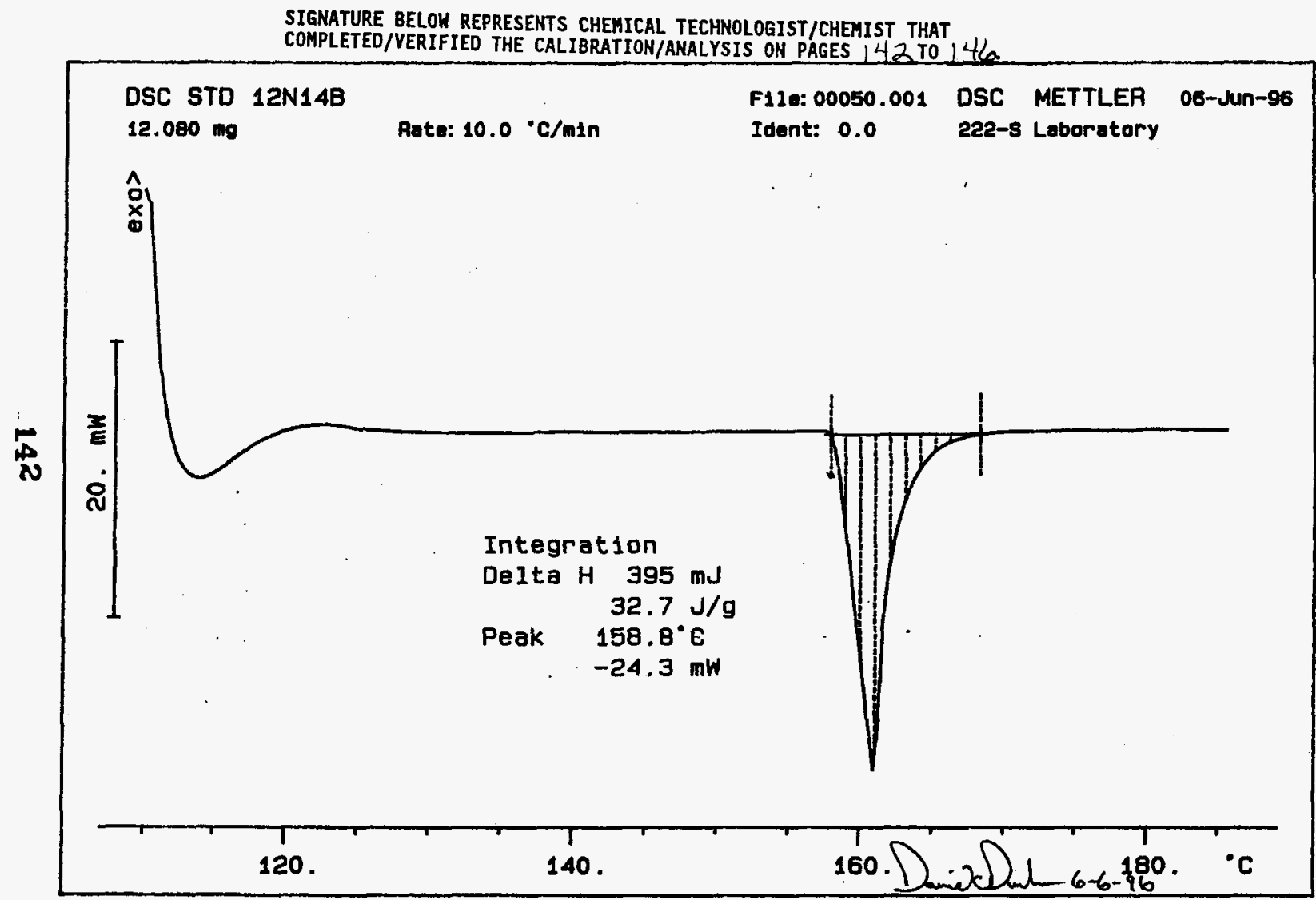




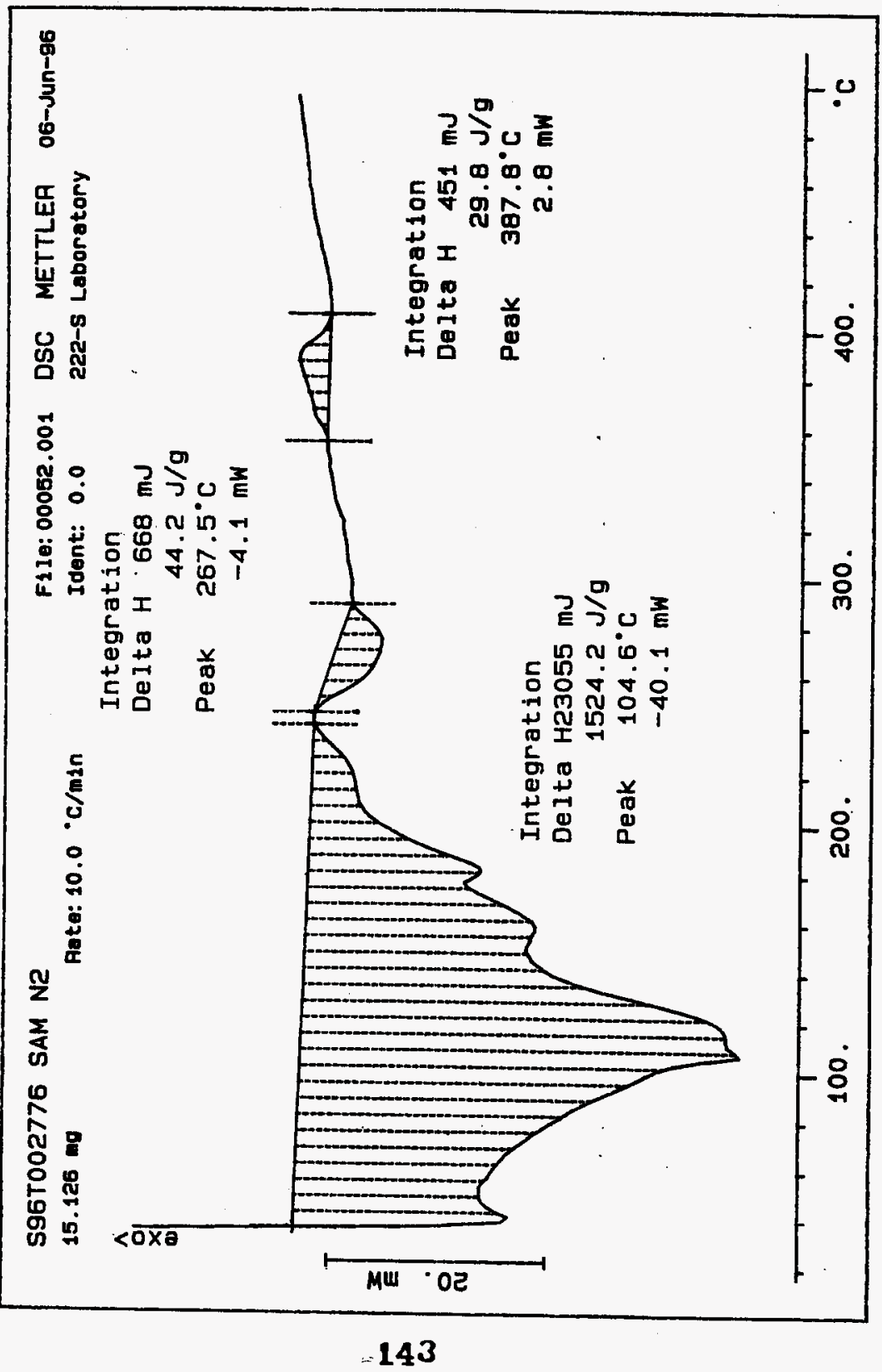




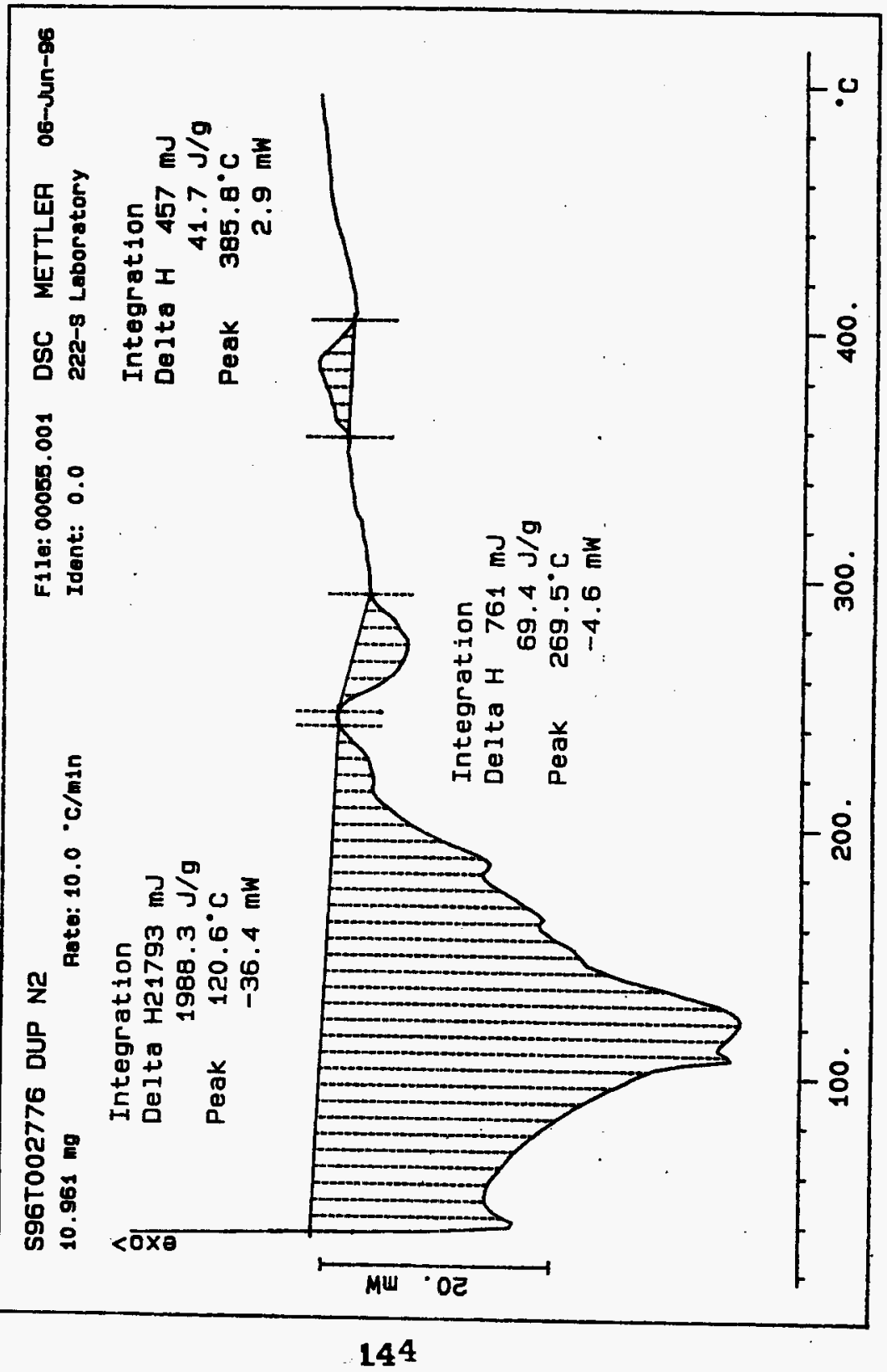




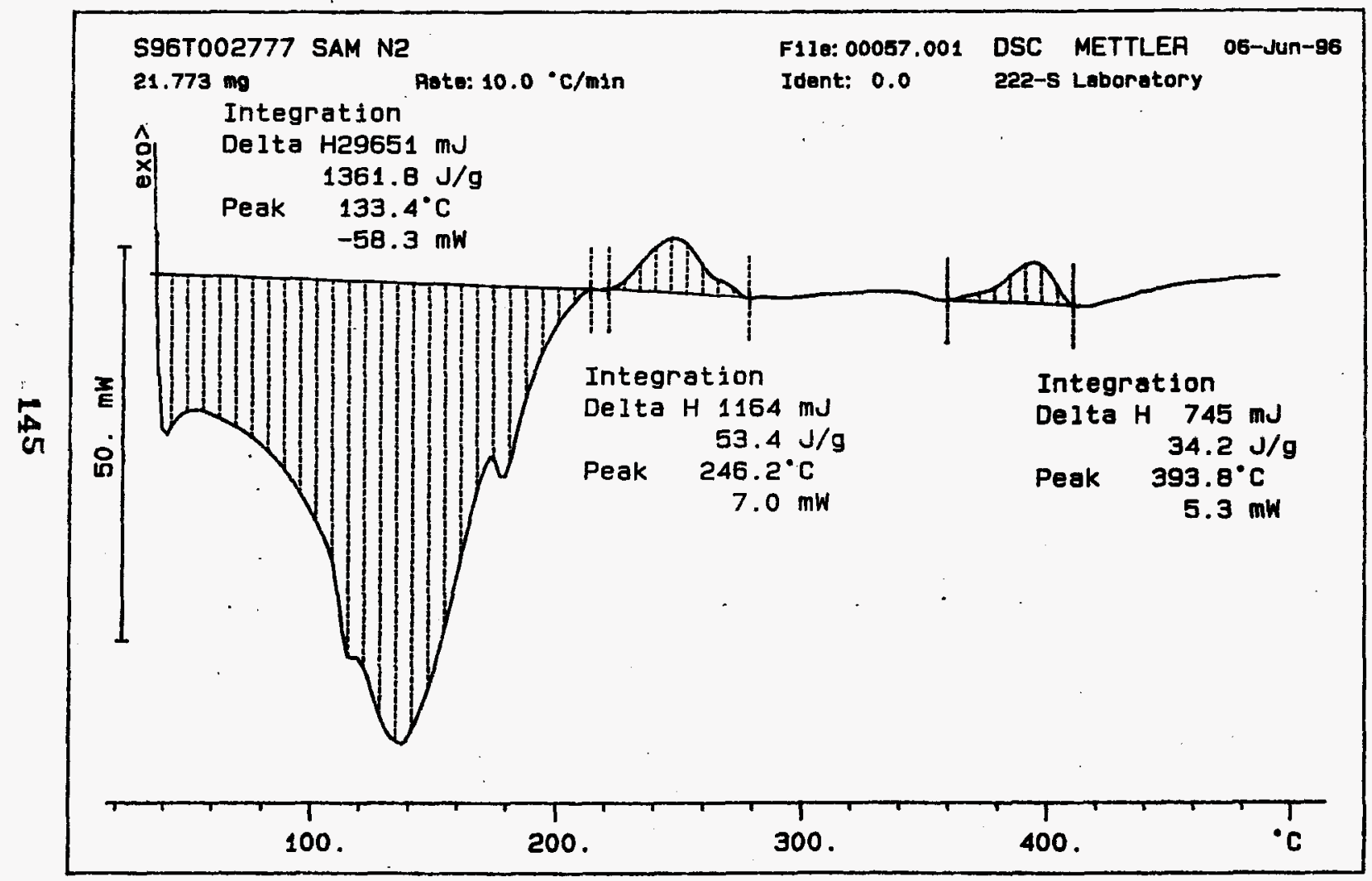




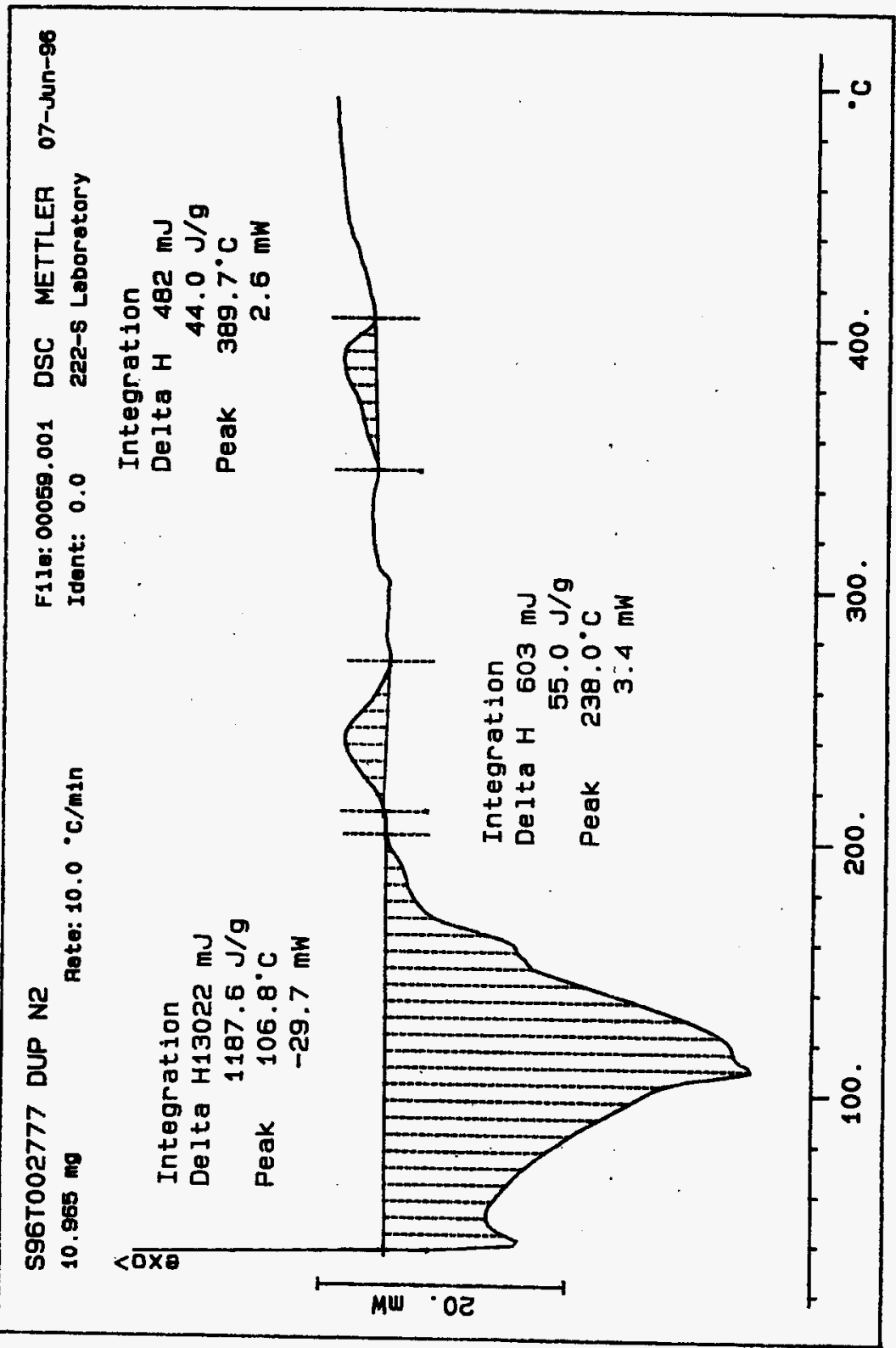


worklistrpt Version $2.105 / 15 / 95$

WHS-SD-WTE-DP-189, REV .O

06/03/96 14:51

LABCORE Data Entry Template for Worklist\#

Page: I

9547

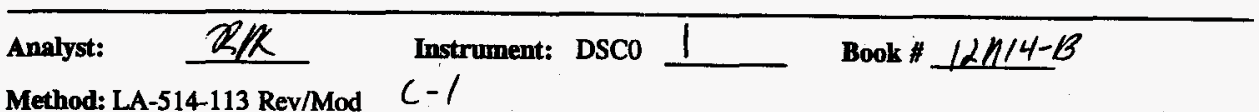

Method: LA-514-113 Rev/Mod C-I

Worklist Comment: U-102 DSC, RUN UNDER N2. RCJ

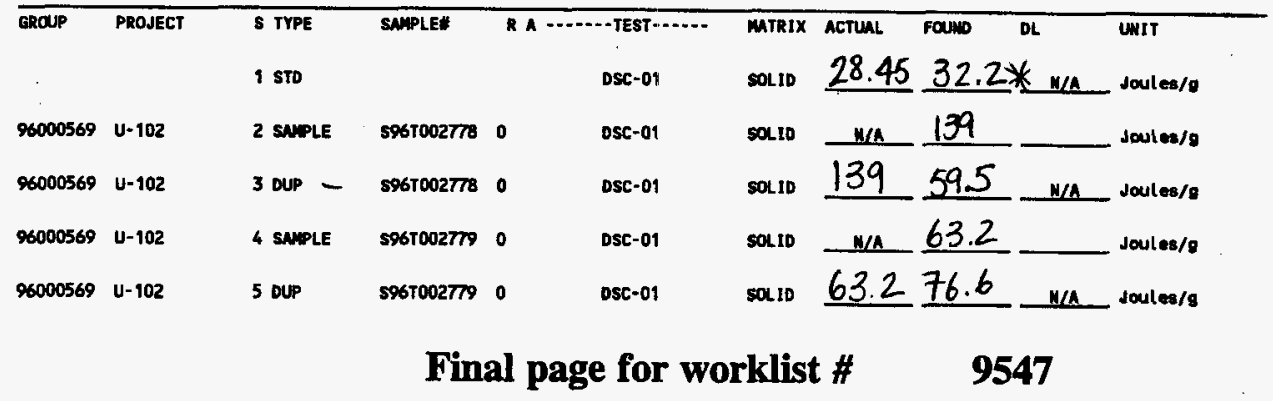

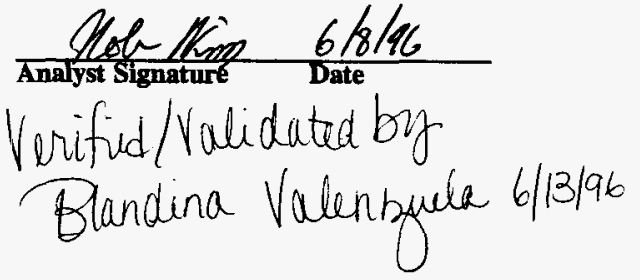

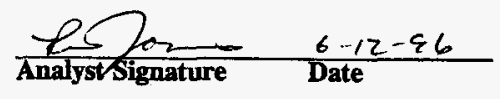

\$967002778 results are the sum of two exotherms. A triplicate want run because results were the close to the notification lima

Data Bury Comments: 596 To 02779 results are the s km: of two exotherms.

Units shown for QC (SPK \& STD) may not reflect the actual units. DL. = Detection Limit, $S=$ Worklist Slot Number, $R .=$ Replicate Number, $A=A$ Aliquot Code.

147 
SIGNATURE ABOVE REPRESENTS CHEMICAL TECHNOLOGIST/CHEMIST THAT

COMPLETED/VERIFIED THE CALIBRATION/ANALYSIS ON PAGES 148 TO 152.

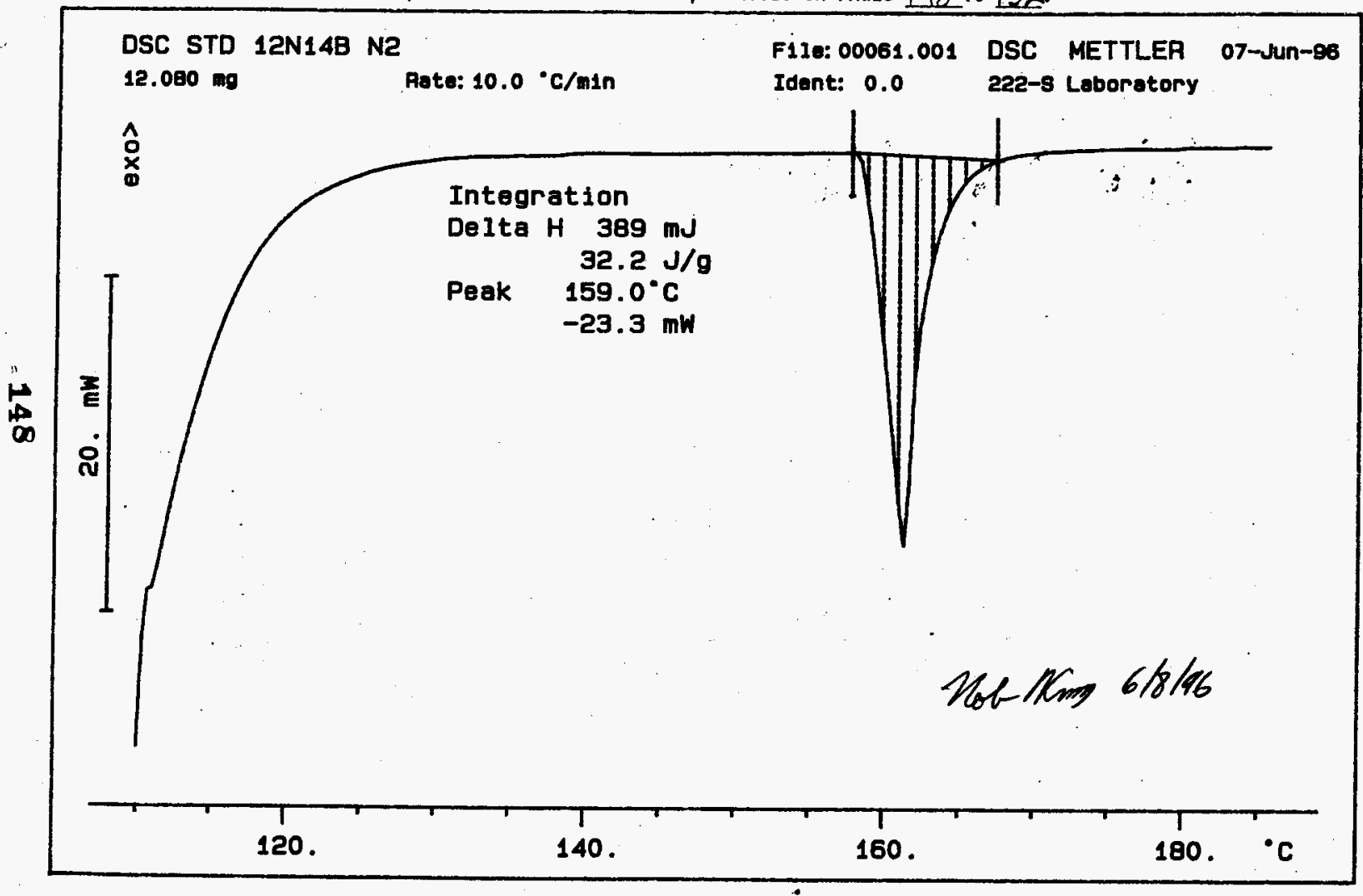


WHC-SU-VIE-DP-189, REV.O

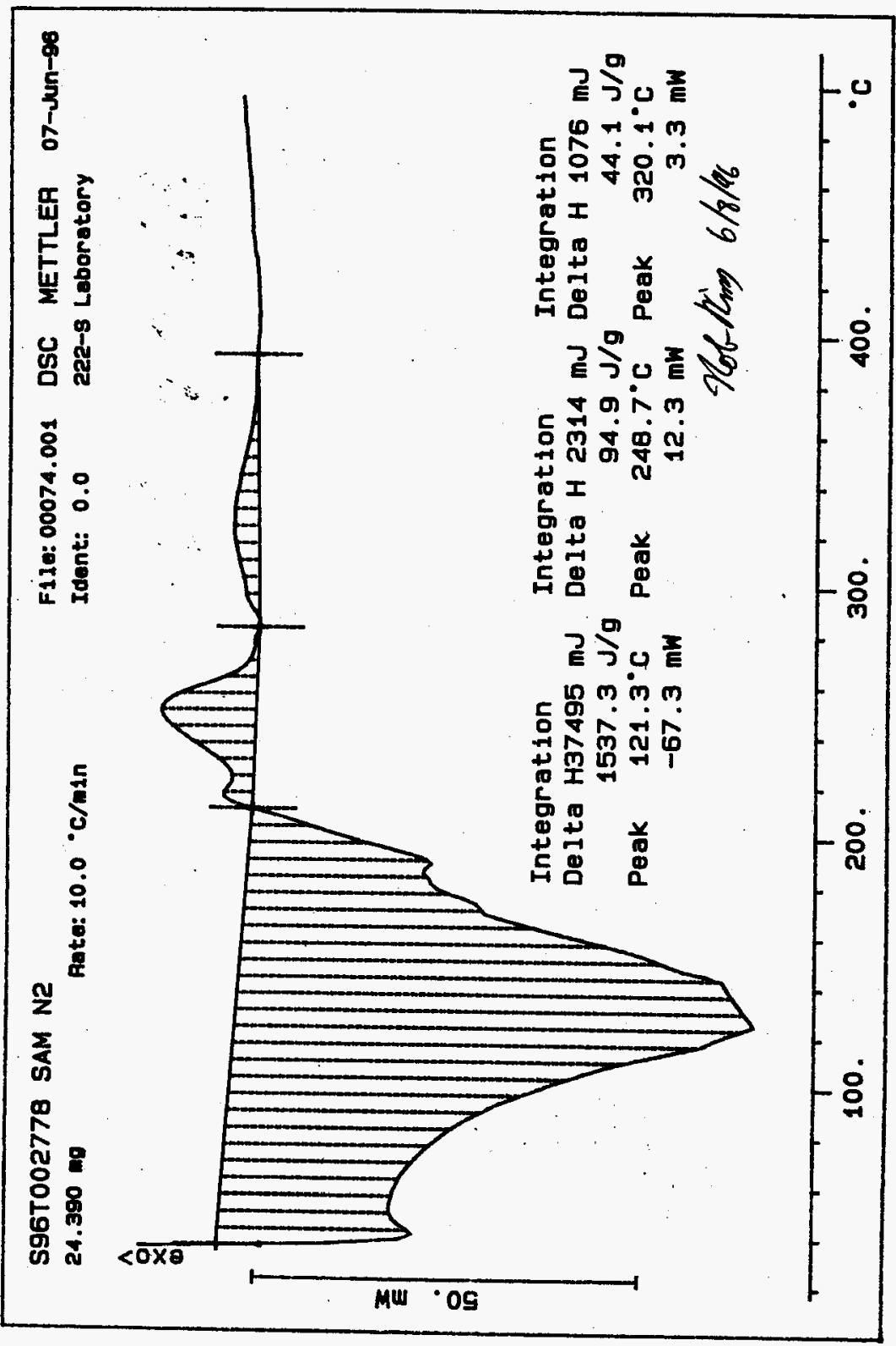




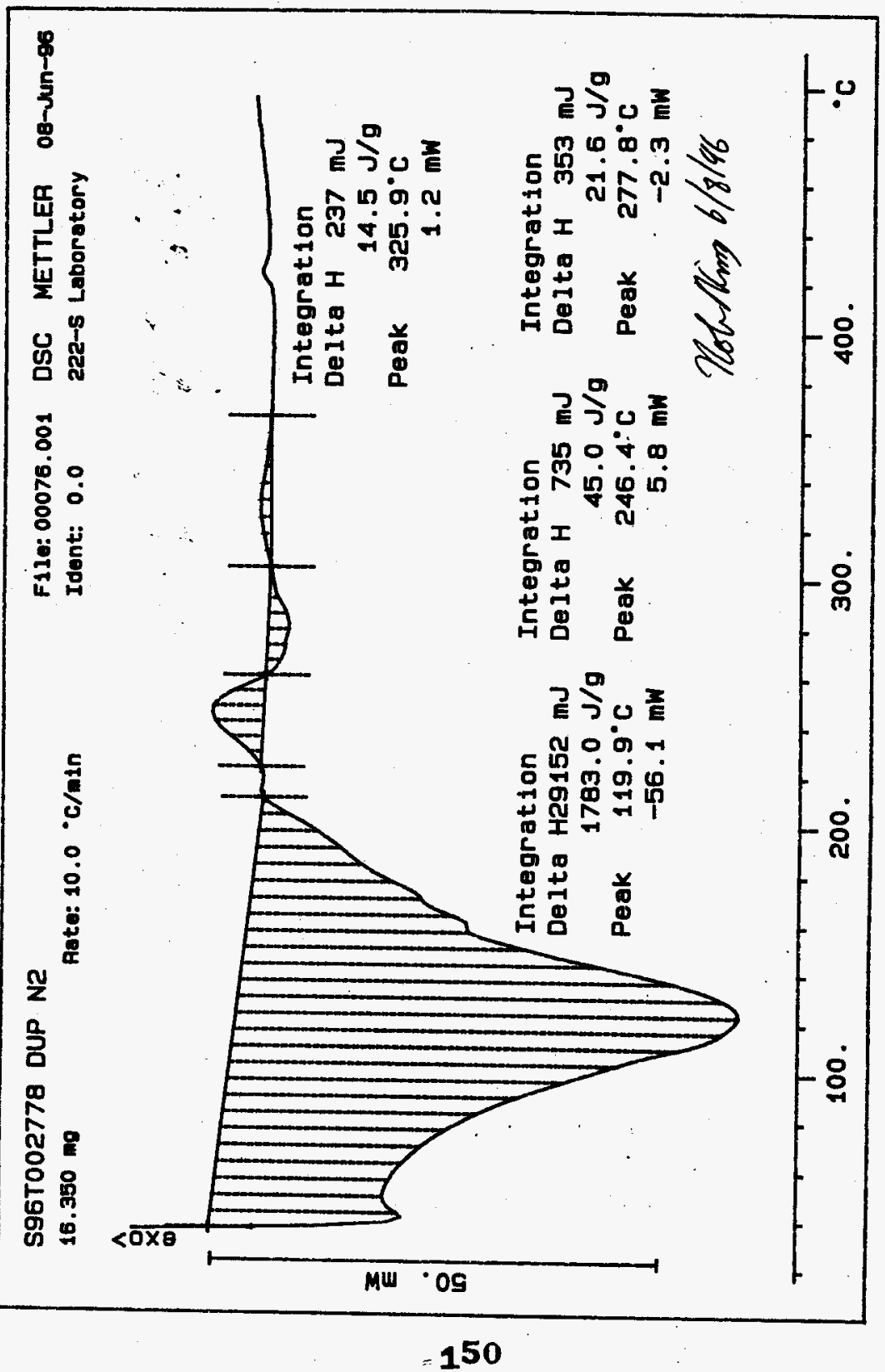


WHIC-SD-WMA-DP-189, REV.O

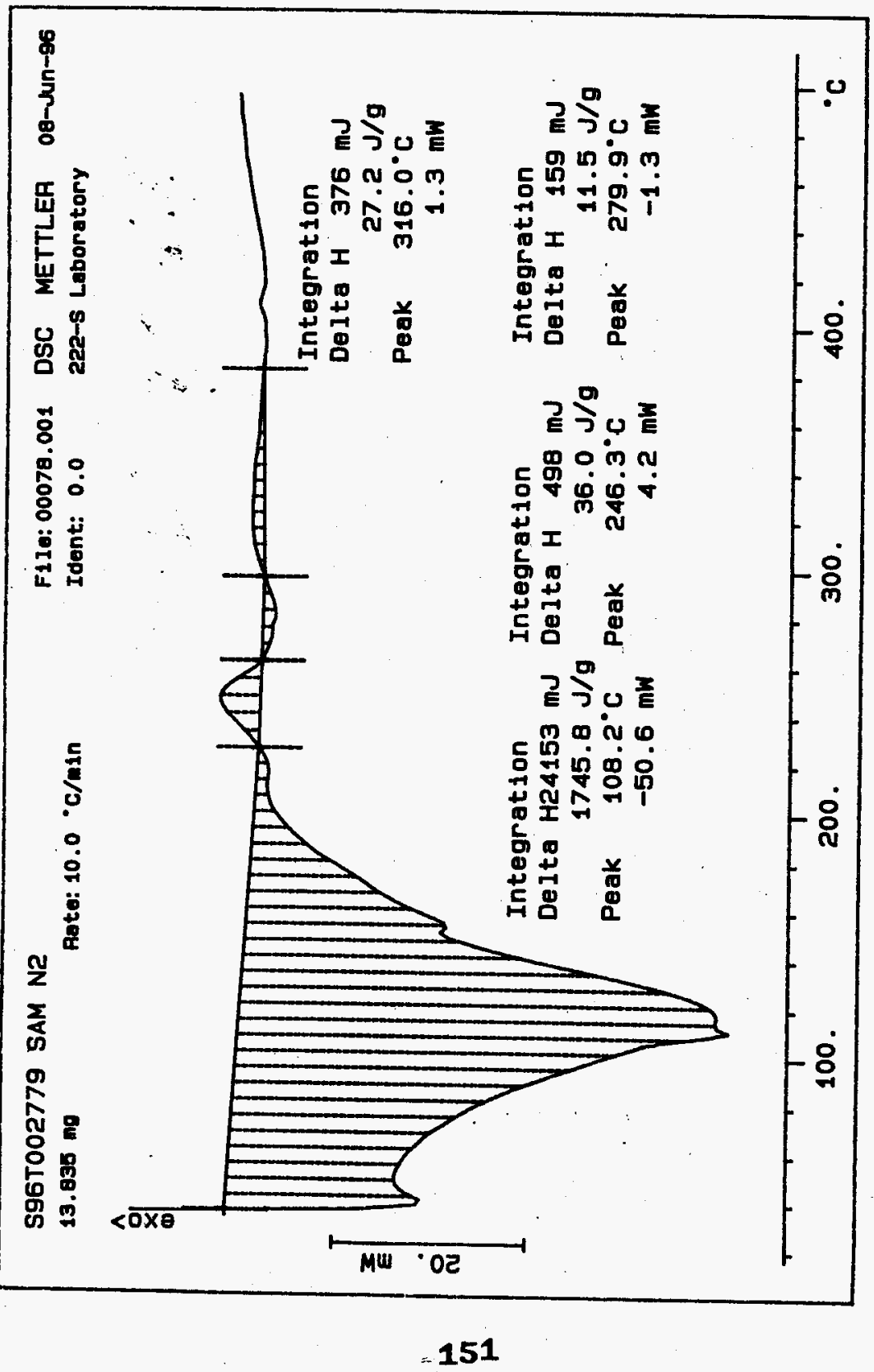


WHIC-SD-WMDP-189, REY. O

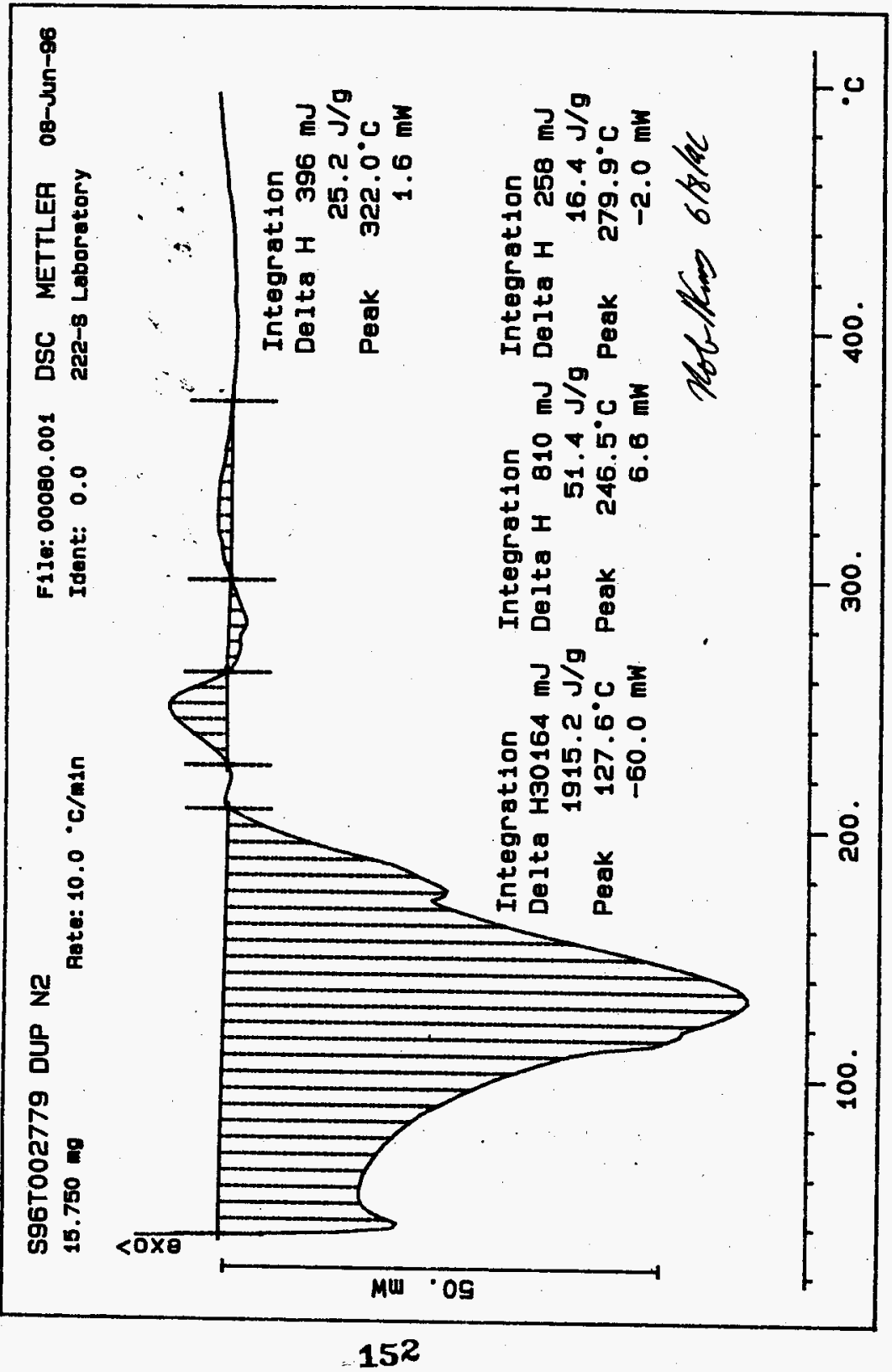




Analyst: DCD Instrument: DSCO I Book \#12N/4B

Method: LA-514-113 Rev/Mod C -

Worklist Comment: U-102 DSC, RUN UNDER N2. RCJ

\begin{tabular}{|c|c|c|c|c|c|c|c|c|c|c|}
\hline GROPP & PROJECT & $\begin{array}{l}S \text { TYPE } \\
9 \mathrm{STD}\end{array}$ & SUMDLE* & & DSC-01 & $\begin{array}{l}\text { MATRIX } \\
\text { SOLID }\end{array}$ & $\begin{array}{l}\text { ActuL } \\
28.45 \\
\end{array}$ & $\begin{array}{l}31.5 \\
\end{array}$ & $\begin{array}{l}\text { DL } \\
. M / A \\
\end{array}$ & $\begin{array}{l}\text { UNIT } \\
\text { Joules/g }\end{array}$ \\
\hline 96000569 & U-102 & 2 SNIPLE & $596 T 002780$ & 0 & DSC-01 & SOL ID & W/A & 14.4 & & Joules/g \\
\hline 96000569 & $u-102$ & 3 oup & 5967002780 & 0 & DSC-01 & SOLID & 4.4 & 19.8 & N/A & Joules/g \\
\hline
\end{tabular}

\section{Final page for worklist \# 9548}

\section{Dinst Signature 6 Date $6-11-96$}

Verified/Validatia by Blandina Valen iquela
Rllted $6-13-16$

Data Entry Comments:

Units shown for $Q C$ (SPK \& STD) may not reflect the actual units. $D L=$ Detection Limit, $S^{\circ}=$ Wontlist Slot Number, $R=$ Replicate Number, $A=$ Aliquot Code. 
SIGNATURE BELON REPRESENTS CHEMICAL TECHNOLOGIST/CHEMIST THAT

COMPLETED/VERIFIED THE CALIBRATION/ANALYSIS ON PAGES 154 TO 156

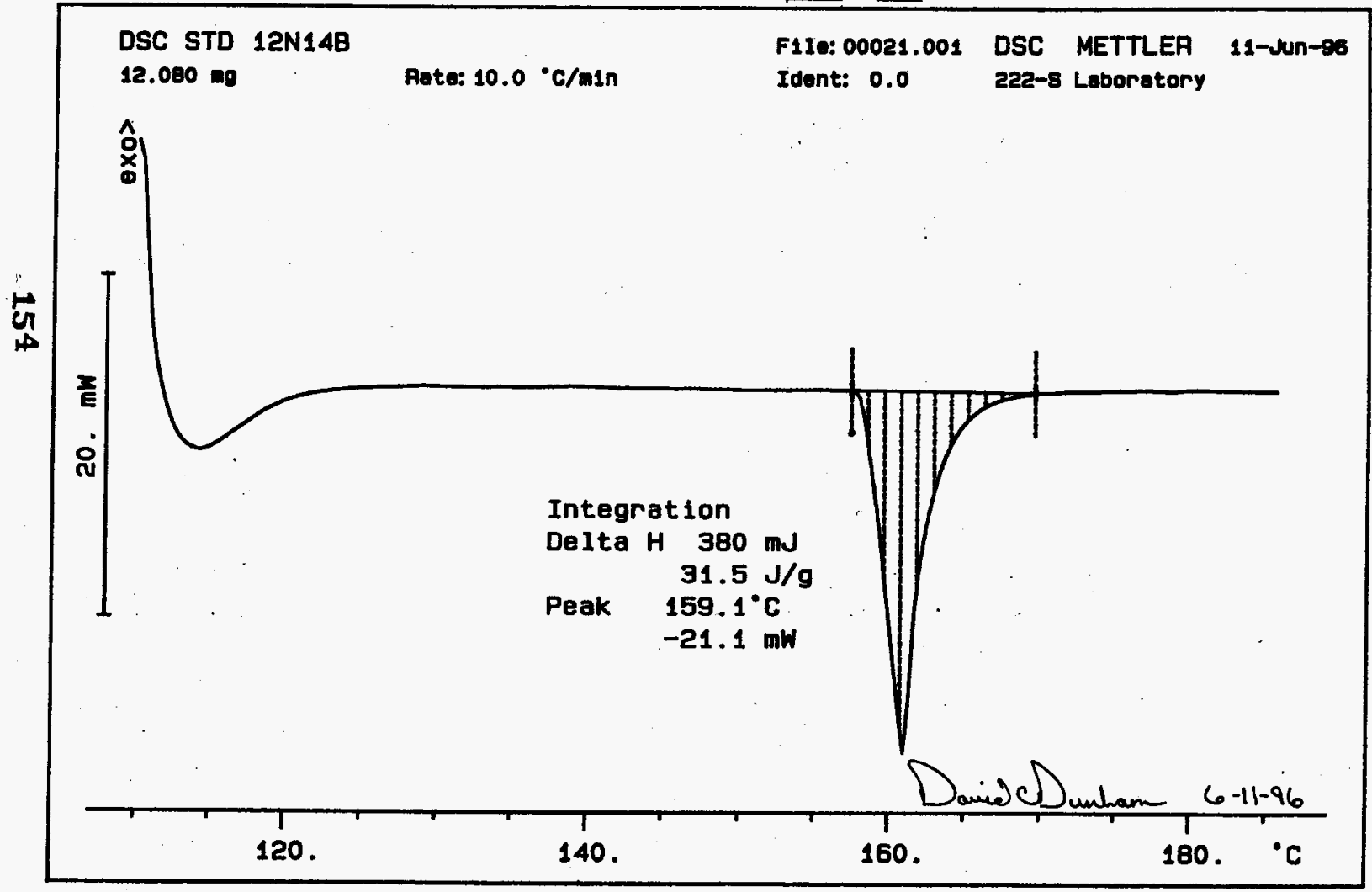




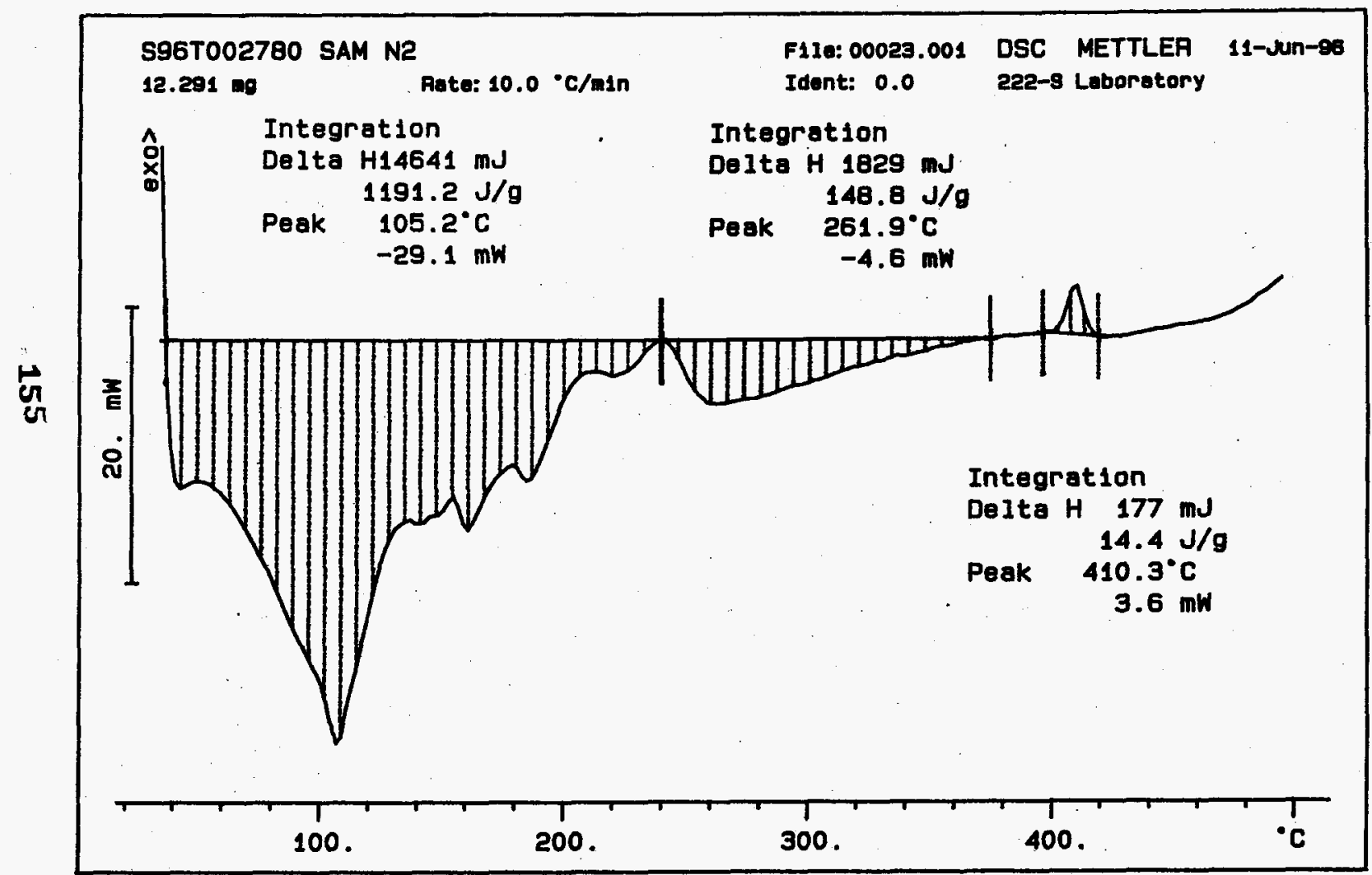




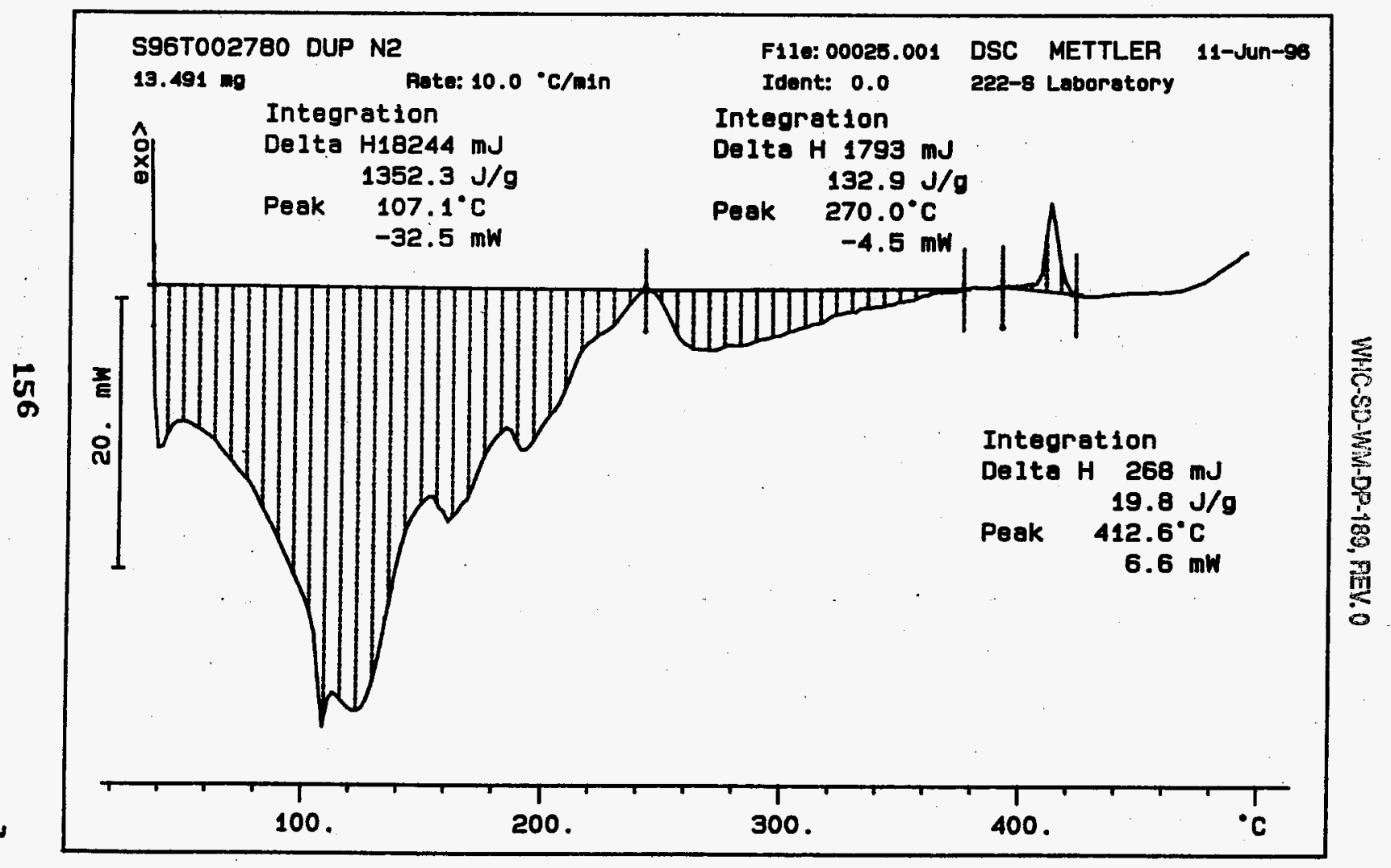




\section{LABCORE Data Entry Template for Worklist\#}

Analyst: EAL Instrument: DSCO 3 Book \# 12 N14B

Method: LA-514-114 Rev/Mod C - I

Worklist Comment: DSC U-102 Reruns Run under N2. RUSH

\begin{tabular}{|c|c|c|c|c|c|c|c|c|c|}
\hline GROUP & PROJECT & $\begin{array}{l}\text { S TYPE } \\
\text { I STD }\end{array}$ & SAMPLE* & & $\begin{array}{c}\text { A } \cdots \text { TEST } \cdots \cdots \\
\text { DSC }-03\end{array}$ & $\begin{array}{l}\text { MATRIX } \\
\text { SOLID }\end{array}$ & $\begin{array}{l}\text { Actual } \\
28.45 \\
\end{array}$ & 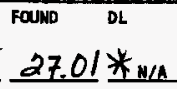 & $\begin{array}{l}\text { UNIT } \\
\text { Joules/R }\end{array}$ \\
\hline 96000536 & $U \cdot 102$ & 2 SNMPLE & S96T002685 & 1 & DSC-03 & SOLID & M/A & 164.3 & Joules/s \\
\hline 96000536 & $U-102$ & 3 DUP & s96T002665 & 1 & ¿i.bsc-03 & SOLID & 164.3 & 141.5 & Joules/g \\
\hline
\end{tabular}

Final page:for worklist \#

10073

Suattached for signatures

Verified/Validated bor
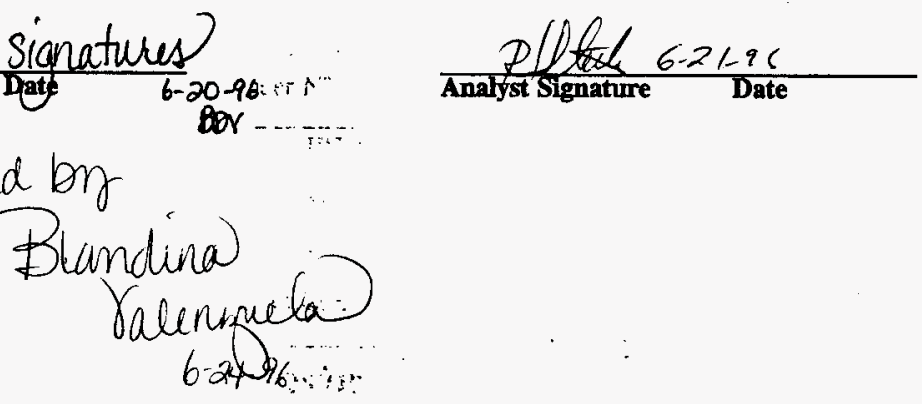

Data Entry Comments:

$\because \because \because$

r....

$\therefore$

Units shown for $Q C$ (SPK \& STD) may not reflect the actual units. $D L=$ Detection Limit, $S=$ Worklist Slot Number,

$R=$ Replicate Number, $A=$ Aliquot Code. 


\section{Analyst: $\quad \& \mathbb{R}$ Instrument: DSC0 \\ Book \# $12 N 14 \cdot B$}

Method: LA-514-113 $\operatorname{Rev} / \mathrm{Mod} C \cdot \mathrm{l}$

Worklist Comment: DSC U-102 Reruns Run under N2. RUSH

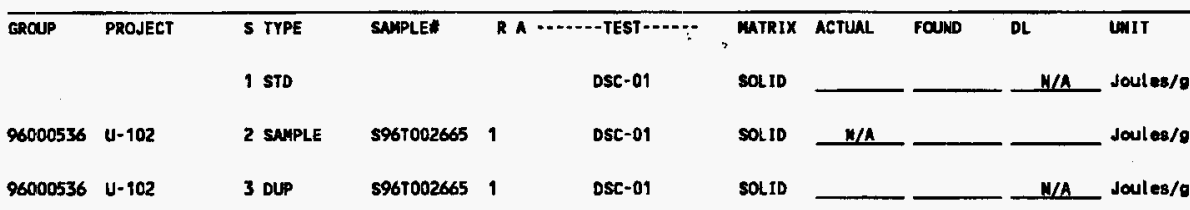

\section{Final page for worklist \# 10073}

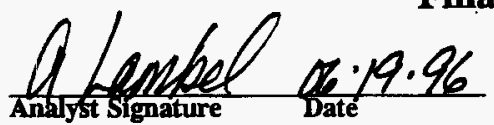

Analyst SIgnature Date

DSC-03 instrument

was noed.

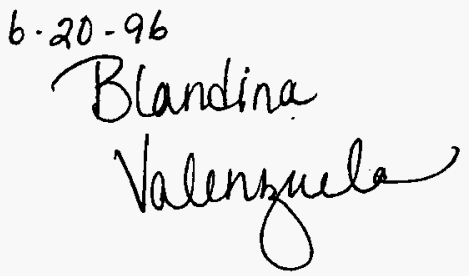

Data Entry Comments:

Units shown for $Q C$ (SPK \& STD) may not reflect the actual writs. $D L=$ Detection Limit, $S=$ Worklist Slot Number, $R=$ Replicate Number, $A=$ Aliquot Code. 


\section{Adambel 06.19 .96}

Curve is DSC

F11e info: Sambiges Mad un 19 05:52 551996

Sample Welght: 12.000 ing

12N14-B INOIUN AT 10CWIN

SIGNATURE ABOVE REPRESENTS CHEMICAL TECHNOLOGIST/CHEMIST THAT

COMPLETED/VERIFIED THE CALIBRATION/ANALYSIS ON PAGES 159 TO $\mathrm{LOL}$.

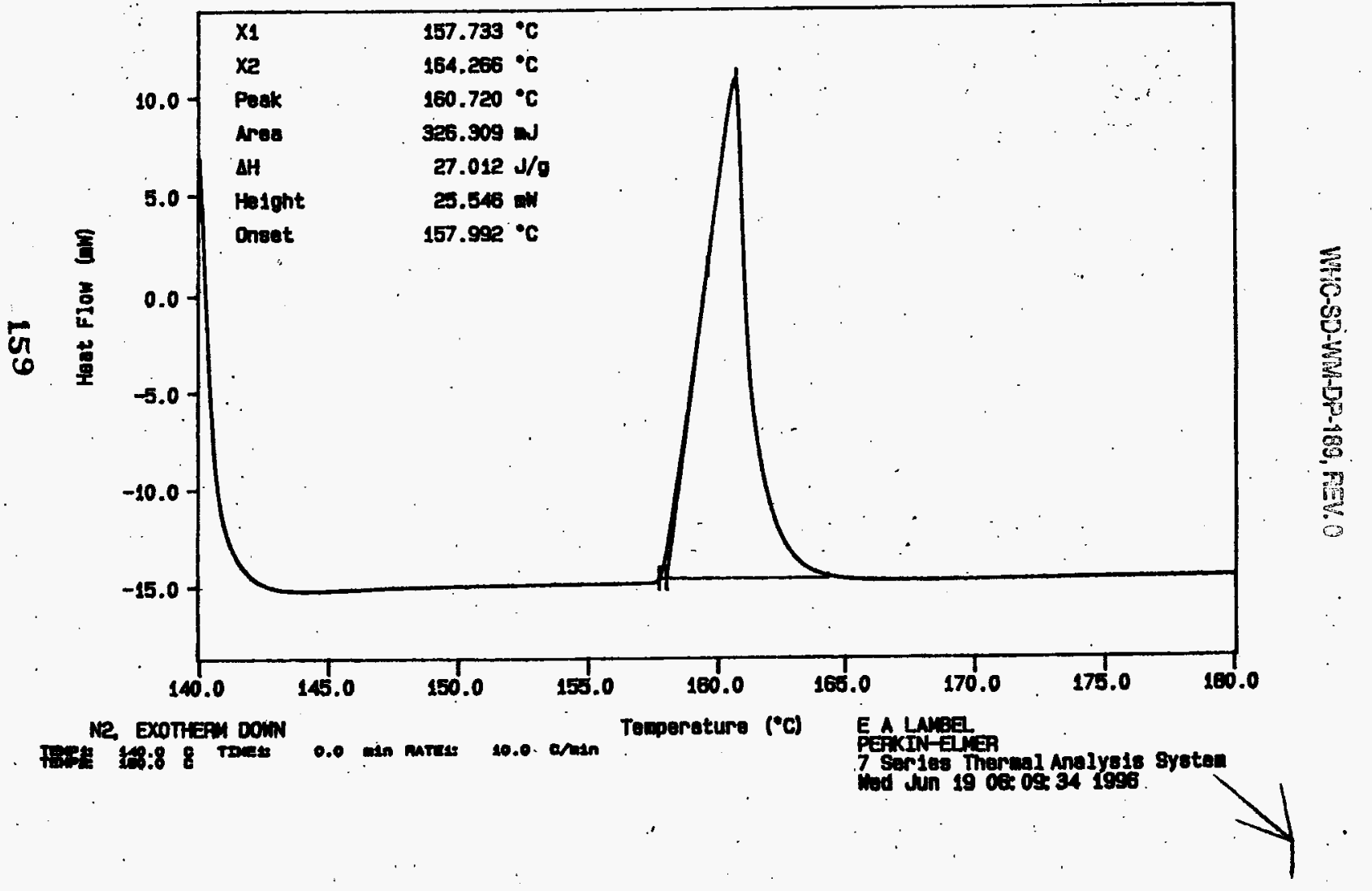


Curve 1: Dec

F1le info: SAm06190s Ned Jun 19 12:00:33 1996

Sample We1ght: 23.120

s967002665

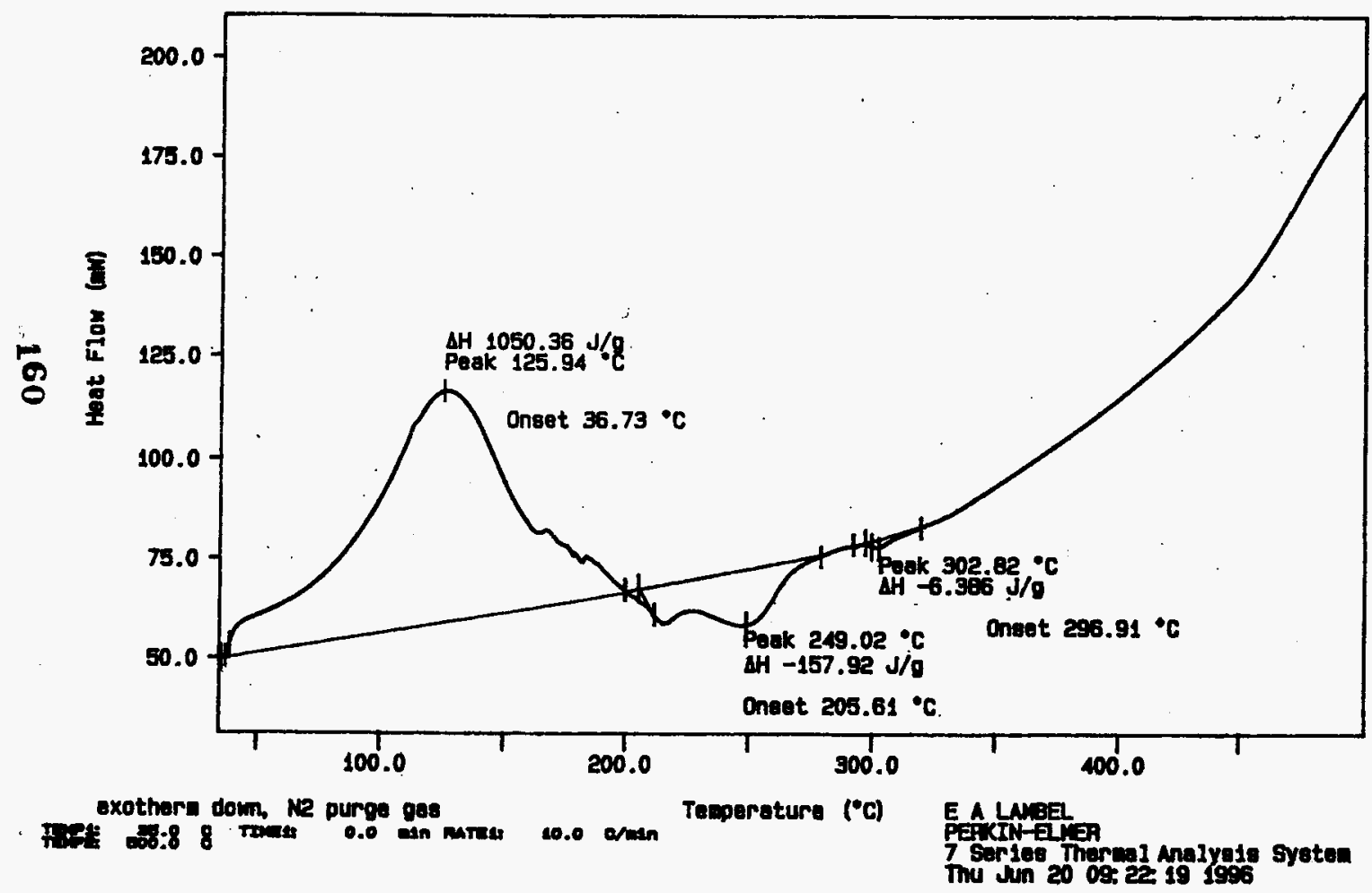


Curve 1: Dec

- F1le 1nfo: SAM061905 Ned un 19 13:52: 461986 Semple Waight: 11.430 mg

S95T002665 DUP

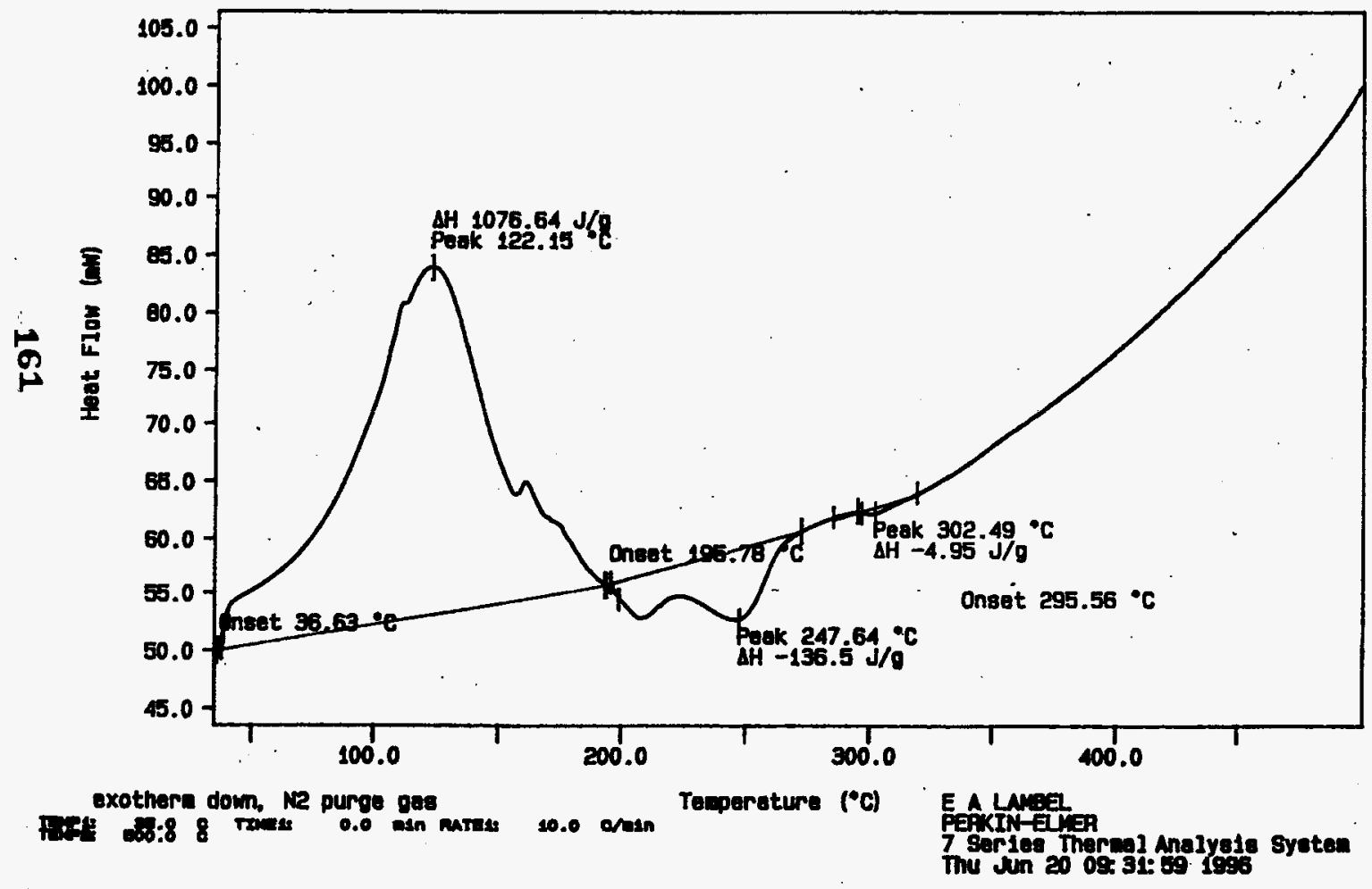


Analyst: He Instrument: DSC01

Book \# N/A

Method: LA-514-113 Rev/Mod C-1

Worklist Comment: U-102 Dry DSCs.

\begin{tabular}{|c|c|c|c|c|c|c|c|c|c|c|}
\hline GROUP & PROJECT & $S$ TYPE & SAMPLE\# & $R A$ & $\because-$ TEST $\cdots . . . .$. & MATRIX & ACTUAL & FOUND & & UNI T \\
\hline 96000569 & U-102 & 1 SAMPLE & 5961002776 & 0 & DSC-02 & SOL10 & N/A & 42.57 & & Joules/g Dry \\
\hline 96000569 & $U-102$ & 2 DUP & 5961002776 & 0 & DSC-02 & SOLID & 42.57 & 59.5 & H/A & Joules $/ g$ Dry \\
\hline 96000569 & $u-102$ & 3 SAMPLE & s96r002777 & o & DSC-02 & SOLID & N/A & 134.4 & & Joules/g Dry \\
\hline 96000569 & $U-102$ & 4 DUP & s96T002777 & 0 & DSC-02 & SOL.1D & 134.4 & 151.8 & N/A & Joules/o Dry \\
\hline 96000569 & $u-102$ & 5 SAMPLE & $596 \mathrm{~T} 002778$ & 0 & DSC. 02 & SOLID & N/A & 226.2 & & Joules/g Dry \\
\hline 96000569 & $U-102$ & 6 DUP & S96T002778 & 0 & DSC- 02 & SOLID & 226.2 & 96.81 & N/A & Joules/g Dry \\
\hline 96000569 & $u-102$ & 7 SAMPLE & 5961002779 & 0 & DSC- 02 & SOLID & $N / A$ & 133.8 & & Joules/g Dry \\
\hline 96000569 & $U-102$ & 8 DUP & S96T002779 & 0 & DSC- 02 & SOLID & 133.8 & 162.2 & N/A & Joules/s Dry \\
\hline 96000569 & $u-102$ & 9 SAMPLE & S96T002780 & 0 & DSC-02 & SOLID & N/A & 25.94 & & Joules/g Dry \\
\hline 96000569 & $U-102$ & 10 DUP & S96T002780 & 0 & DSC-02 & SOL 10 & 25.94 & 35.67 & W/A & Joules/g Dry \\
\hline 96000569 & $u-102$ & 11 SAMPLE & 596T002647 & 0 & DSC-02 & SOLID & H/A & 20.13 & & Joules/g Dry \\
\hline 96000569 & U-102 & 12 DUP & S96T002647 & 0 & DSC-02 & SOLID & 20.13 & 32.55 & N/A & Joules/g Dry \\
\hline 96000569 & $u-102$ & 13 SAMPLE & S96T002775 & 0 & DSC-02 & SOLIO & N/A & 45.17 & & Joules/g Dry \\
\hline 96000569 & U- 102 & 14 DUP & S96T002775 & 0 & DSC -02 & SOL ID & 45.17 & 46.32 & N/A & Joules/g Dry \\
\hline
\end{tabular}

\section{Final page for worklist \# 10099}

\section{NRnastos b-20.96 \\ Analyst Signature Date \\ Validated 6-20.96 kea \\ Analyst Signature Date}

Data Entry Comments:

Units shown for QC (SPK \& STD) may not reflect the actual units. $D L=$ Detection Limit, $S=$ Worklist Slot Number, $R=$ Replicate Number, $A=$ Aliquot Code. 


\section{LABCORE Data Entry Template for Worklist\#}

Analyst: Hea Instrument: DSC01

Book \# N/A

Method: LA-514-113 Rev/Mod C-1

Worklist Comment: U-102 Dry DSCs.

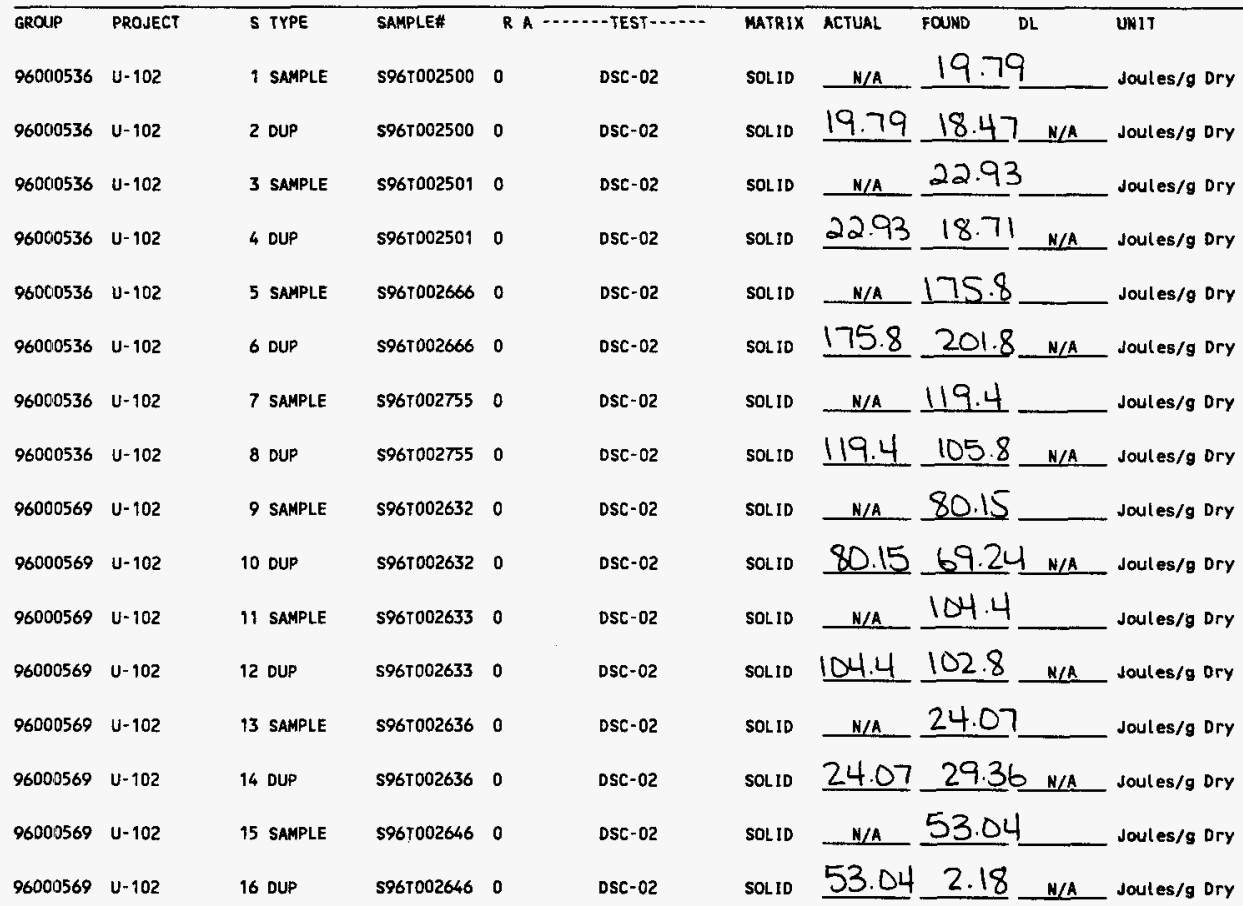

Data Entry Comments:

Units shown for $Q C$ (SPK \& STD) may not reflect the actual units. $D L=$ Detection Limit, $S=$ Worklist Slot Number, $R=$ Replicate Number, $A=$ Aliquot Code. 


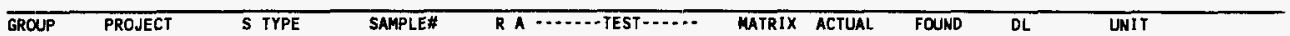

\section{Final page for worklist \# 10100}

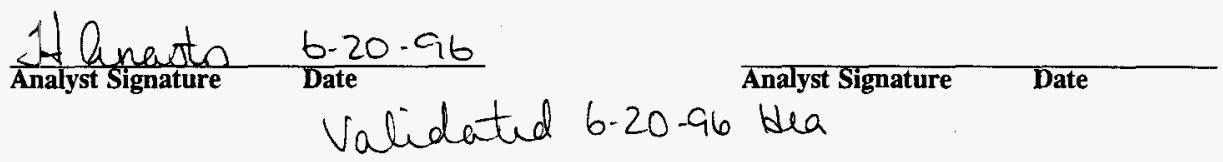

Units shown for $Q C$ (SPK \& STD) may not reflect the actual units. $D L=$ Detection Limit, $S=$ Worklist Slot Number, $R=$ Replicate Number, $A=$ Aliquot Code. 


\section{Analyst: \\ Hea \\ Instrument: DSC01 \\ Book \# N/A}

Method: LA-514-113 Rev/Mod $\mathrm{C}-1$

Worklist Comment: U-102 Dry DSCs

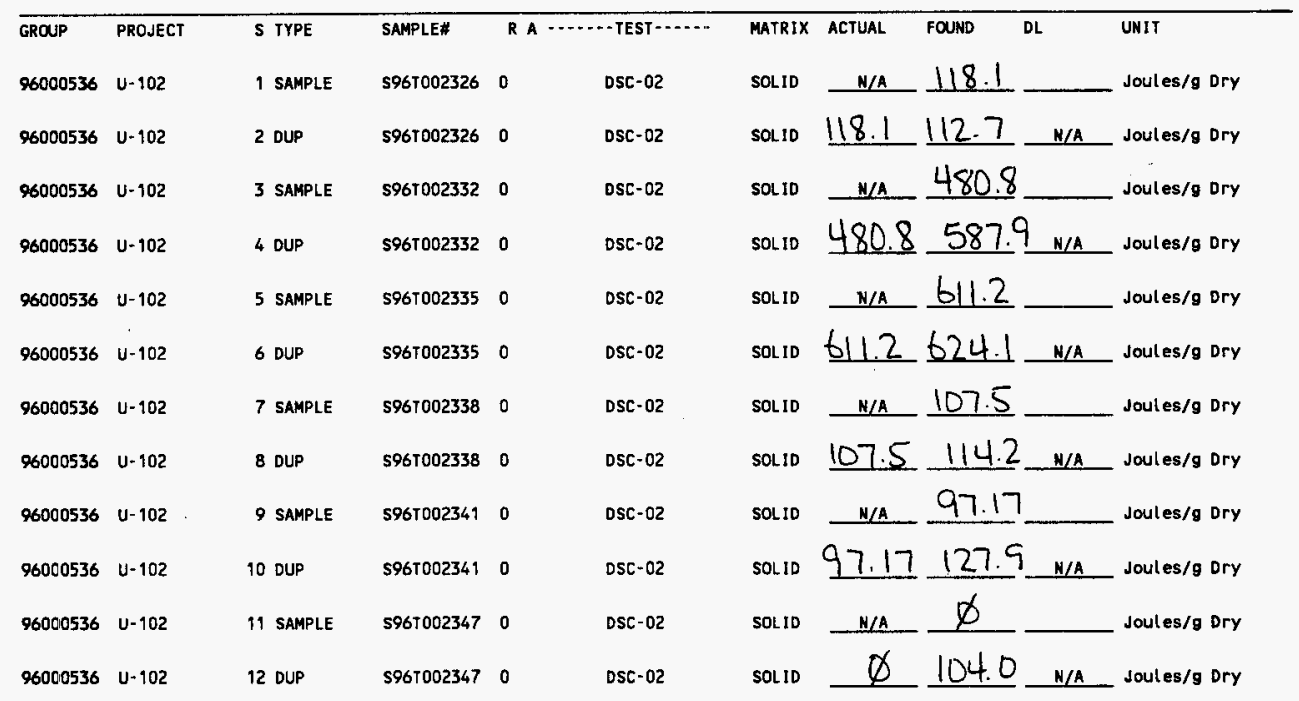

Final page for worklist \# 10101

Nanaston b-20-96

Analyst Signature Date

Validated 6-20-96 tha

Analyst Signature Date

Data Entry Comments:

Units shown for QC (SPK \& STD) may not reflect the actual units. $D L=$ Detection Limit, $S=$ Worklist Slot Number,

$R=$ Replicate Number, $A=$ Aliquot Code. 


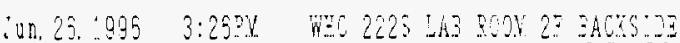

worklistpt Version 2.1 05/15/105

MPo-

No. 2453 P. 1/2

06/20/96 14:15

\section{LABCORE Data Entry 'Template for Worklist\#}

Page: I

10117

Analyst: $\quad$ In $\quad$ Instrument: DSC01 Book \#-

Method: LA-514-113 Rev/Mod

Worklist Comment: Dry DSC for U-102. bdy

\begin{tabular}{|c|c|c|c|c|c|c|c|c|c|}
\hline GROUP & PROJECT & 5 TYPE & SAMPLE: & RA & -TEST- - - ... & MTRIX & ACTUAL & DL & UNIT \\
\hline 96000536 & U-102 & 1 SAPLE & $596 T 002329$ & 0 & DSC-02 & SOLID & 109.6 & & Joules/\& Dry \\
\hline 96000536 & U-102 & 2 DUP & S96T002329 & o & DSC-02 & SOLJD & 109.6106 .8 & w/a & Joules/g Dry \\
\hline 96000536 & U- 102 & 3 SNPLE & S96T002346 & 0 & DSC-02 & SOL1D & $\varnothing$ & & Joules/g Dry \\
\hline 96000536 & $U-102$ & 4 OUP & 5961002344 & 0 & DSC-02 & SOLID & 15.45 & N/A & Joules/g Dry \\
\hline 9600536 & $v-102$ & 5 TRIPL & $\$ 967002344$ & 0 & DSC-02 & SOLID & 10.63 & N/A & Joules/o Dry \\
\hline 96000536 & U-102 & 6 SAMPLE & 5967002665 & 0 & DSC-02 & SOLID & N/A 305.0 & - & Joules/g Dry \\
\hline 96000536 & $U-102$ & 7 DUP & S96T002665 & 0 & DSC-02 & SOLID & 30501 & N/A & Joules/a Dry \\
\hline 96000536 & $U=102$ & 8 SAMPLE & S967002665 & 1 & Dsc-02 & Sol 10 & 258.5 & & Joules/g Dry \\
\hline 96000536 & $U-102$ & 9 DUP & 5967002665 & 1 & DSC-02 & SOLID & 258.5 & N/A & Joules/g Dry \\
\hline 96000536 & $U-102$ & 10 SAMAPLE & 596T002323 & 0 & DST-02 & LIOUID & 246.8 & & Joules/g Dry \\
\hline 96000536 & $U-102$ & 11 DUP & 5967002323 & 0 & DSC -02 & LIOUID & $24 t .8 \quad 251.3$ & H/A & Joules/g Dry \\
\hline 96000569 & $U-102$ & 12 SAMPLE & 5967002549 & 0 & DSC-02 & LIOUID & 07.5 & & Joules/g Dry \\
\hline 961000569 & U-102 & 13 DUP & $5 \% 67002549$ & 0 & DSC-02 & LIOUID & 207.5272 .8 & $N / A$ & Joules/g Dry \\
\hline 96000569 & $U=102$ & 14 SNPLLE & S96T002762 & 0 & DSC-02 & LIOUID & $H / A$ & & Joules/g Dry \\
\hline 96000569 & U-102 & 15 DUP & $\$ 967002762$ & 0 & DSC-02 & LIQUID & $\phi$ & W/A & Joules/g Dry \\
\hline
\end{tabular}

Final page for worklist \# 10117

Data Entry Comments:

Units shown for $Q C$ (SPK \& STD) moy not reflect the actual units. $D L=$ Detection Limit, $S=$ Worklist Slot Number, $R=$ Replicate Number, $A=$ Aliquot Code. 
wortlistrpt Version 2.1.05/15/95

Page: 2

06/20/96 14:15

\section{LABCORE Data Entry Template for Worklist\#}

10117

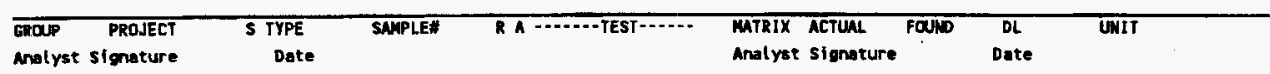

WHC-SD-WW-DP-16\%, FEV.O

Units shown for QC (SPK \& STD) may not reflect the actual units. $D L=$ Detection Limit, $S=$ Worklist Slot Number, $R=$ Replicate Number, $A=$ Aliquot Code. 


Analyst: ROMn Instrument: TGAO $\quad$ Book \# $82 \mathrm{NgA}$

Method: LA-560-112 Rev/Mod B-

Worklist Comment: U-102 TGA RUN UNDER N2. RCJ

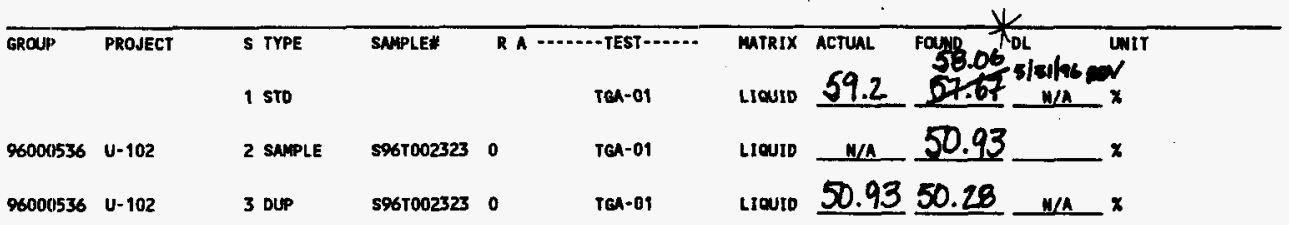

\section{Final page for worklist \# 9248}

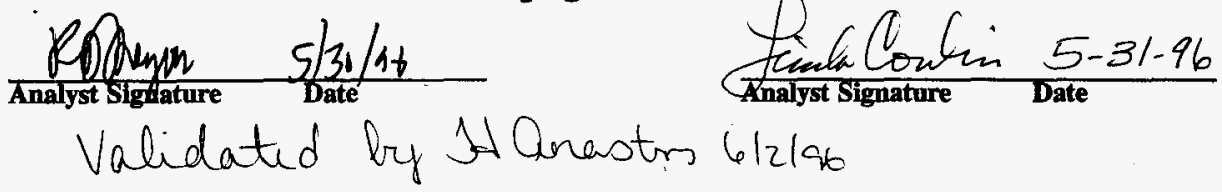

Data! Entry Comments:

Units shown for QC (SPK \& STD) may not reflect the actual units. $D L=$ Detection Limit, $S=$ Worklist Slot Number, $R=$ Replicate Number, $A=$ Aliquot Code. 


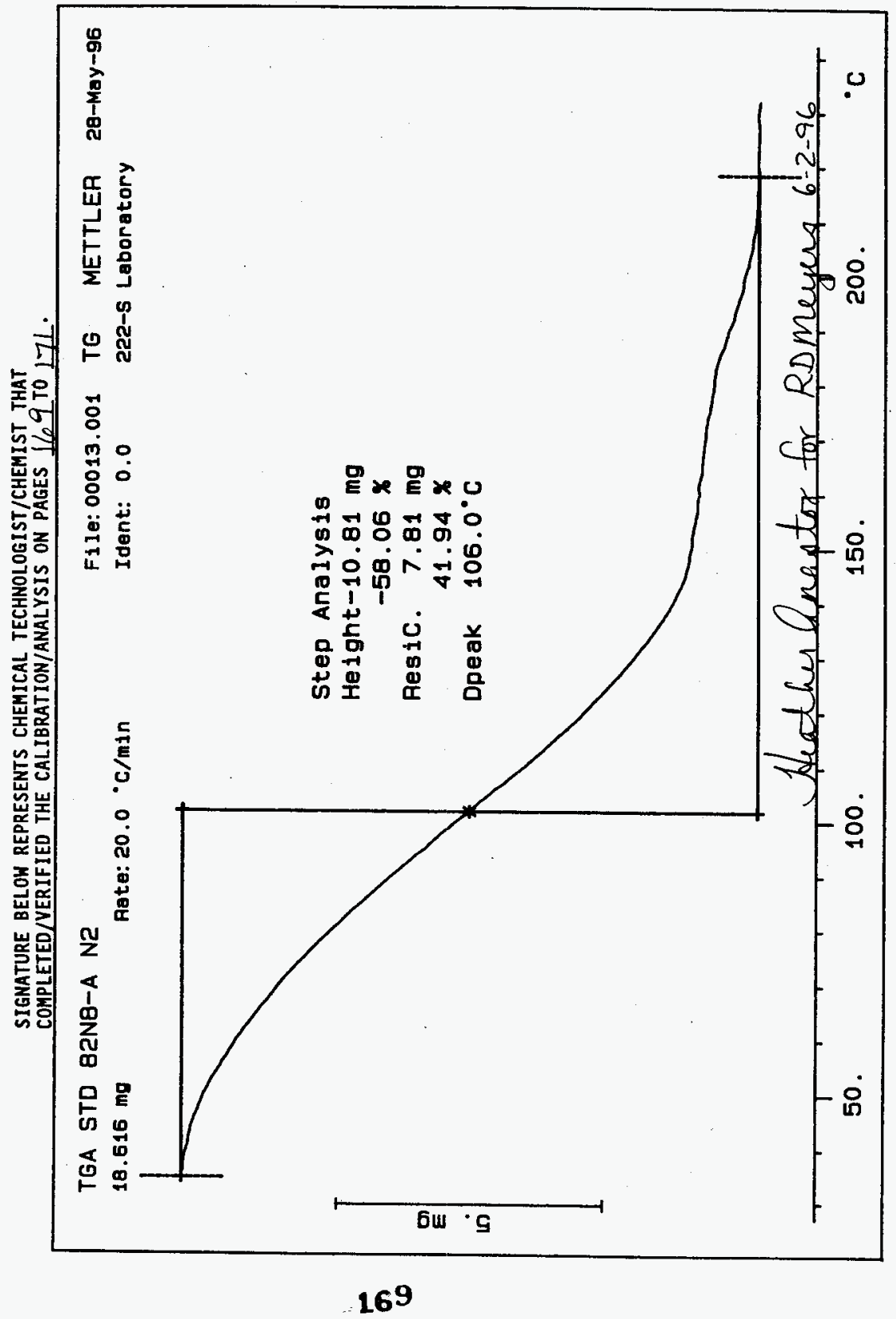




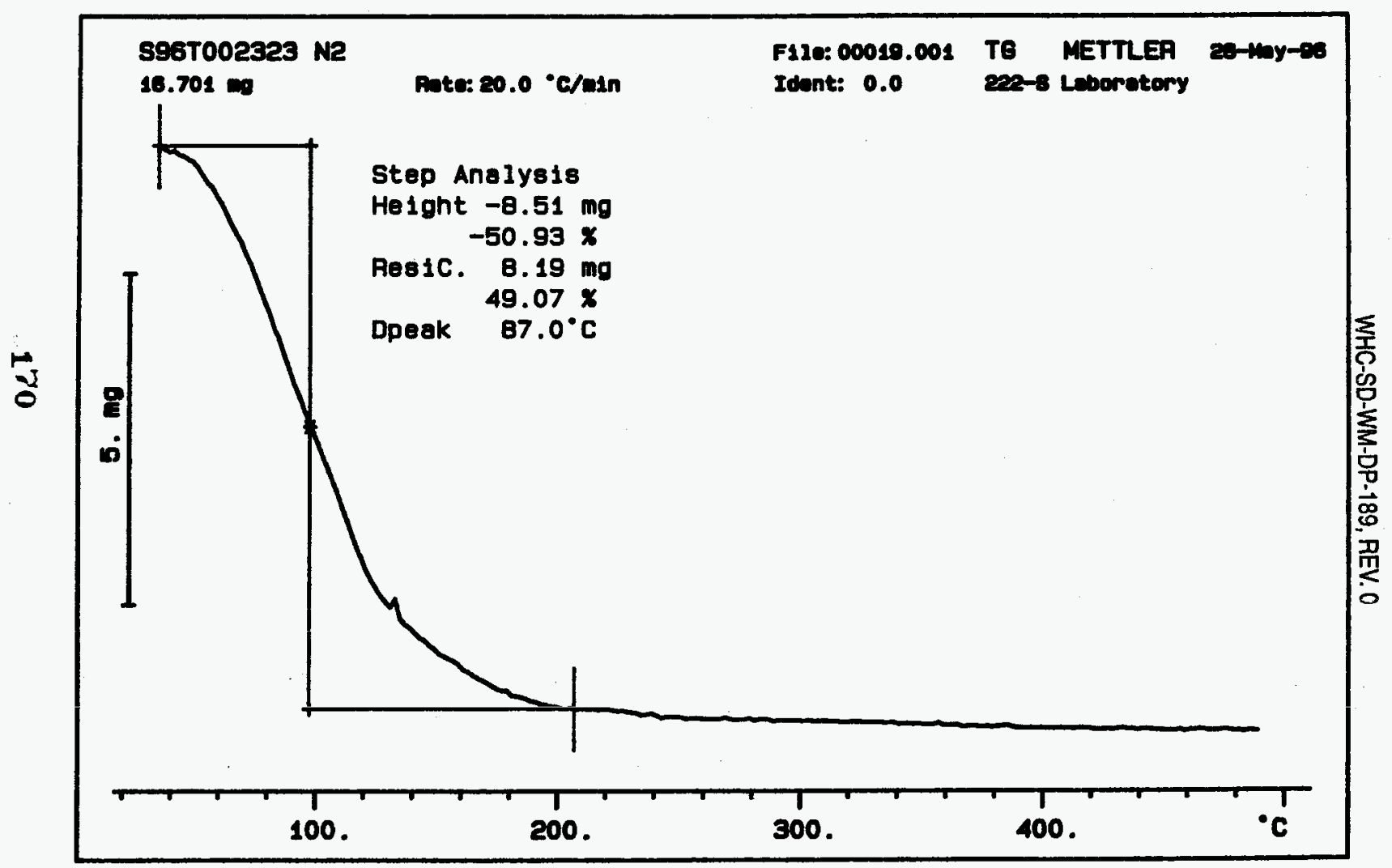




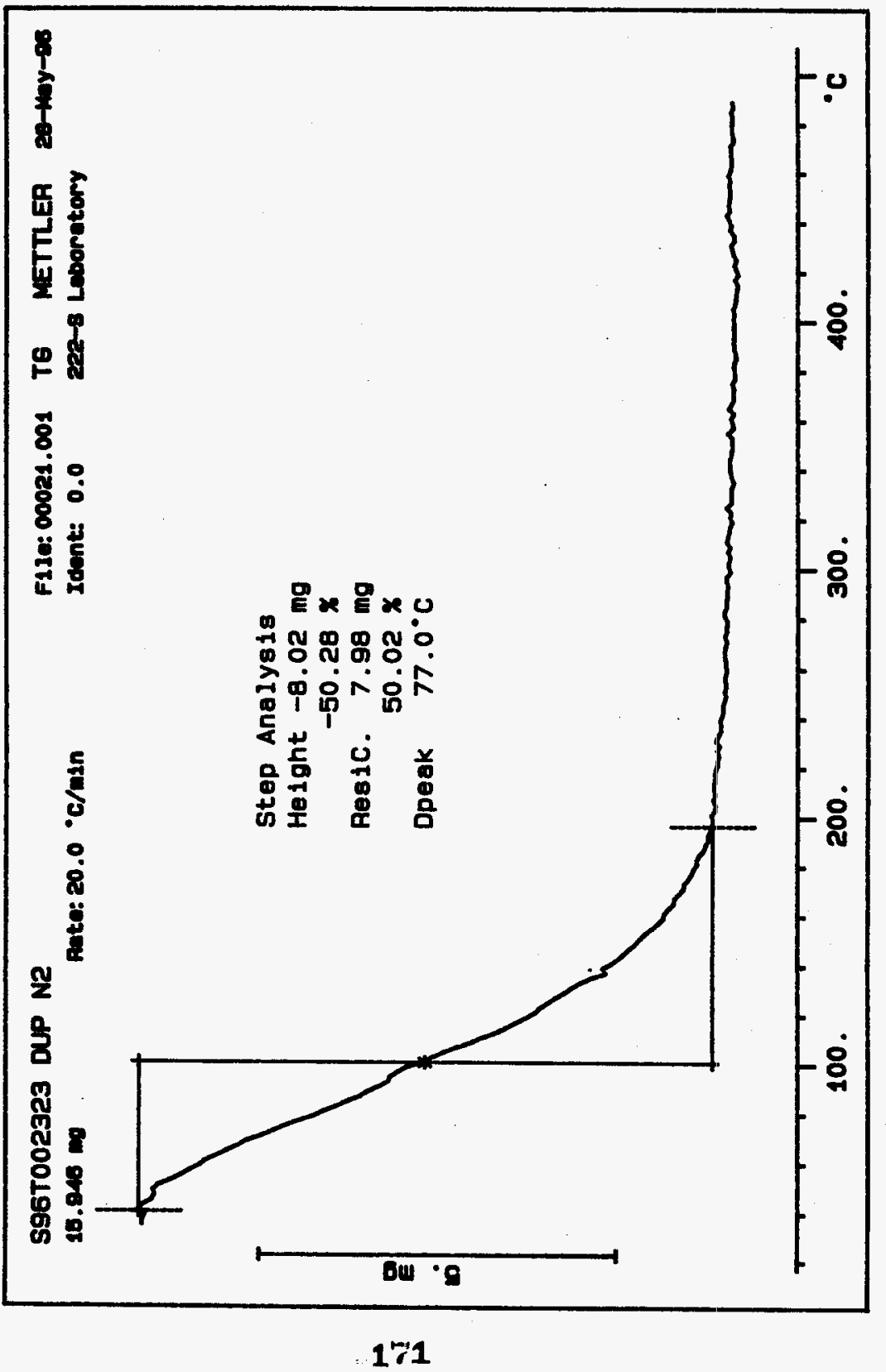




Analyst: PJM Instrument: TGA0 3 Book \# 82 N8,A

Method: LA-514-114 Rev/Mod C-1

Worklist Comment: U-102 TGA RUN UNDER N2. RCJ

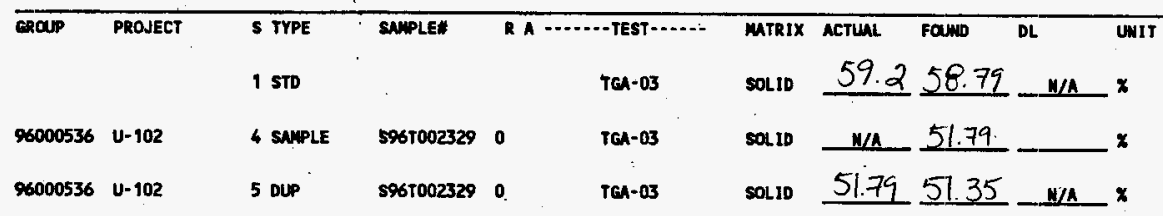

\section{Final page for worklist \#}

Bandina Valentulefor DPRTMcloun Analyst Signature stente Anayst Signature

\section{9}


Curve 1: TBA

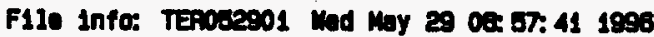

8ompe waight: 23.083 in

ToA $8 T 0$ seins-A

SIGNATURE BELOW REPRESENTS CHEMICAL TECHNOLOGIST/CHEMIST THAT

COMPLETED/VERIFIED THE CALIBRATION/ANALYSIS ON PAGES 173 TO 175.

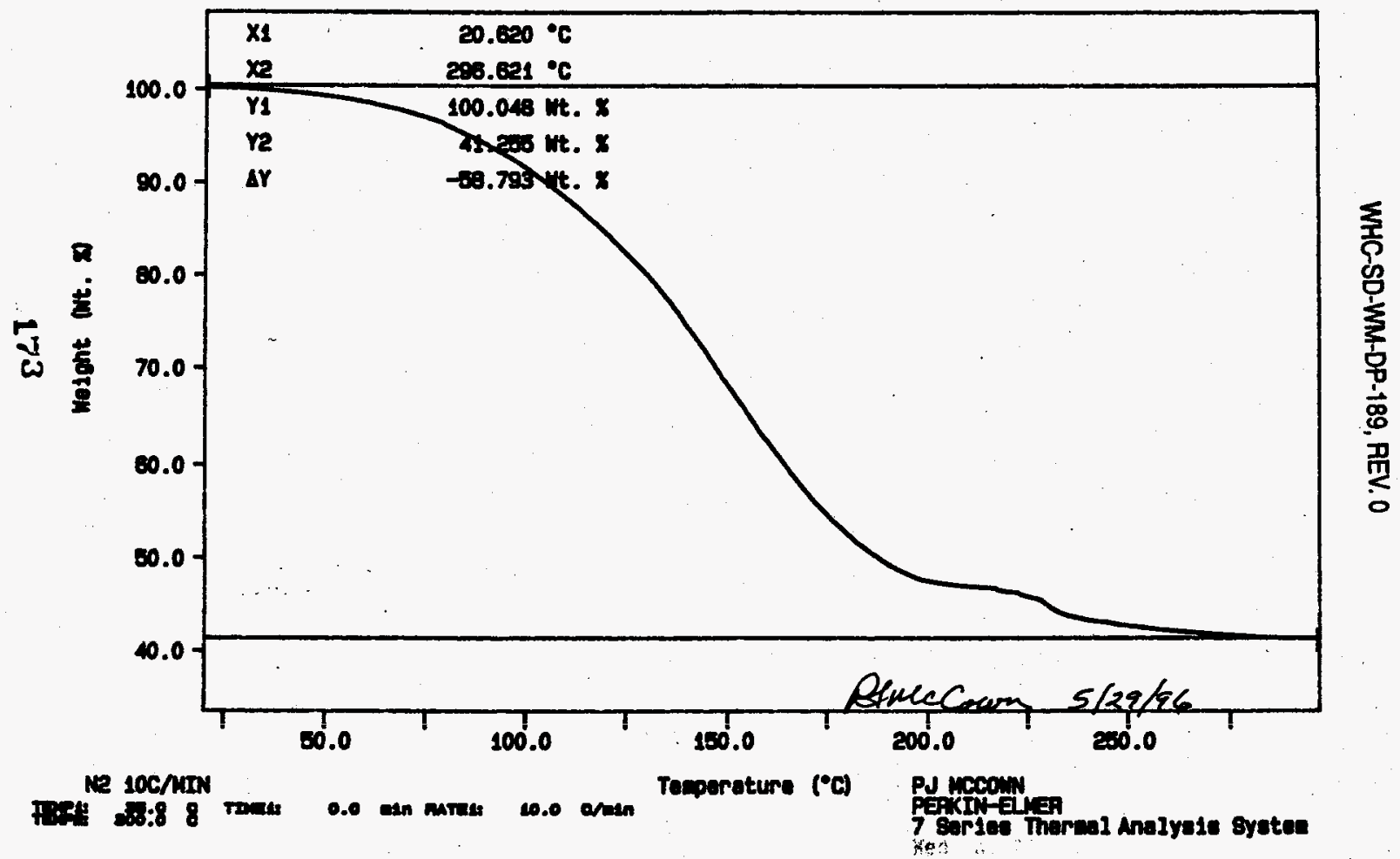


Curve 1: TGA

F1le info: SAM052907 Hed May 29 22: 36: 571996

Sanple Weight: 13.661

S96T002329

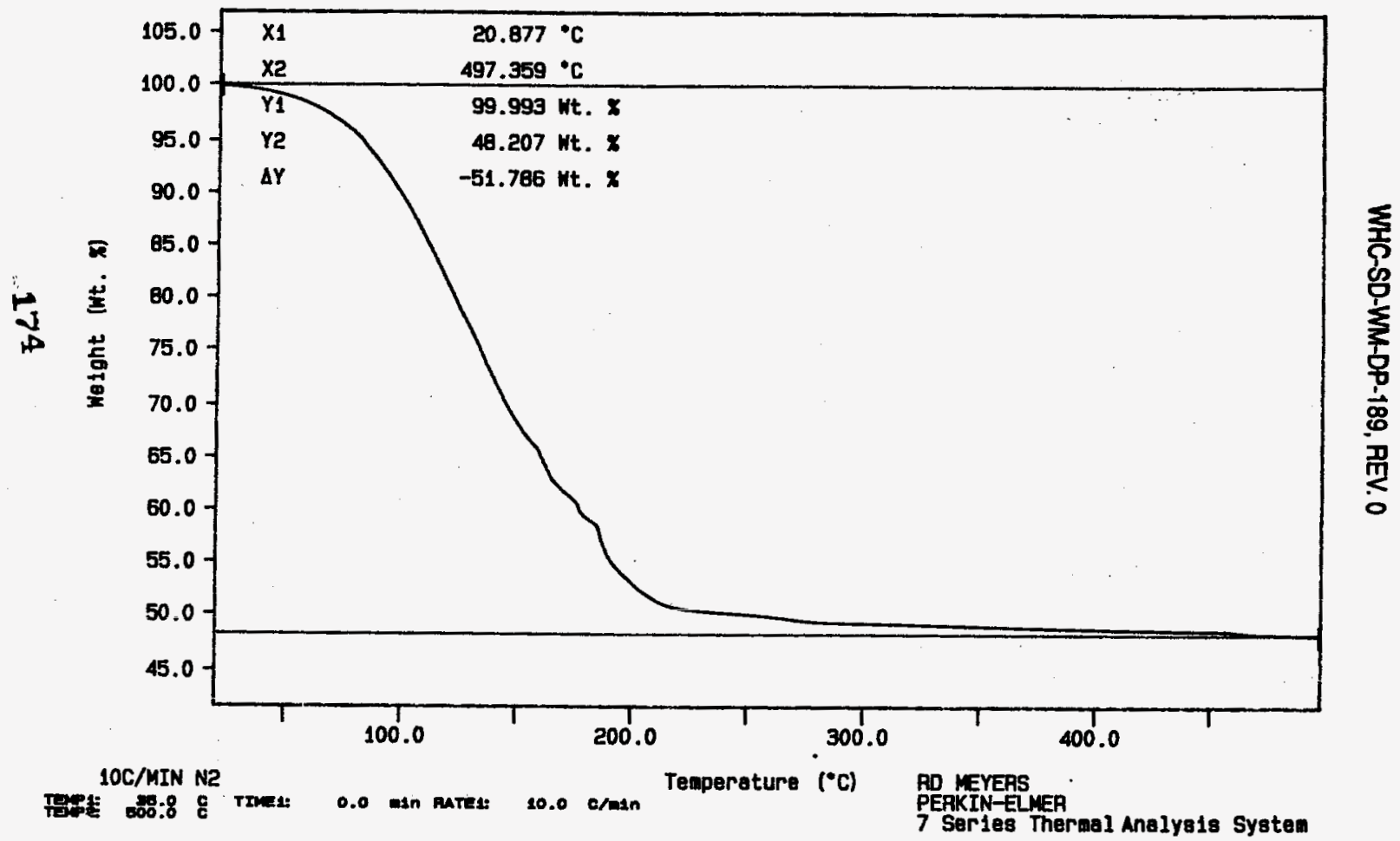


Curve 1: TGA

F1le info: SAm053001 Thu May 30 02: 04: 321996

Sample We1ght: 11.674

ing

S96T0023290uP

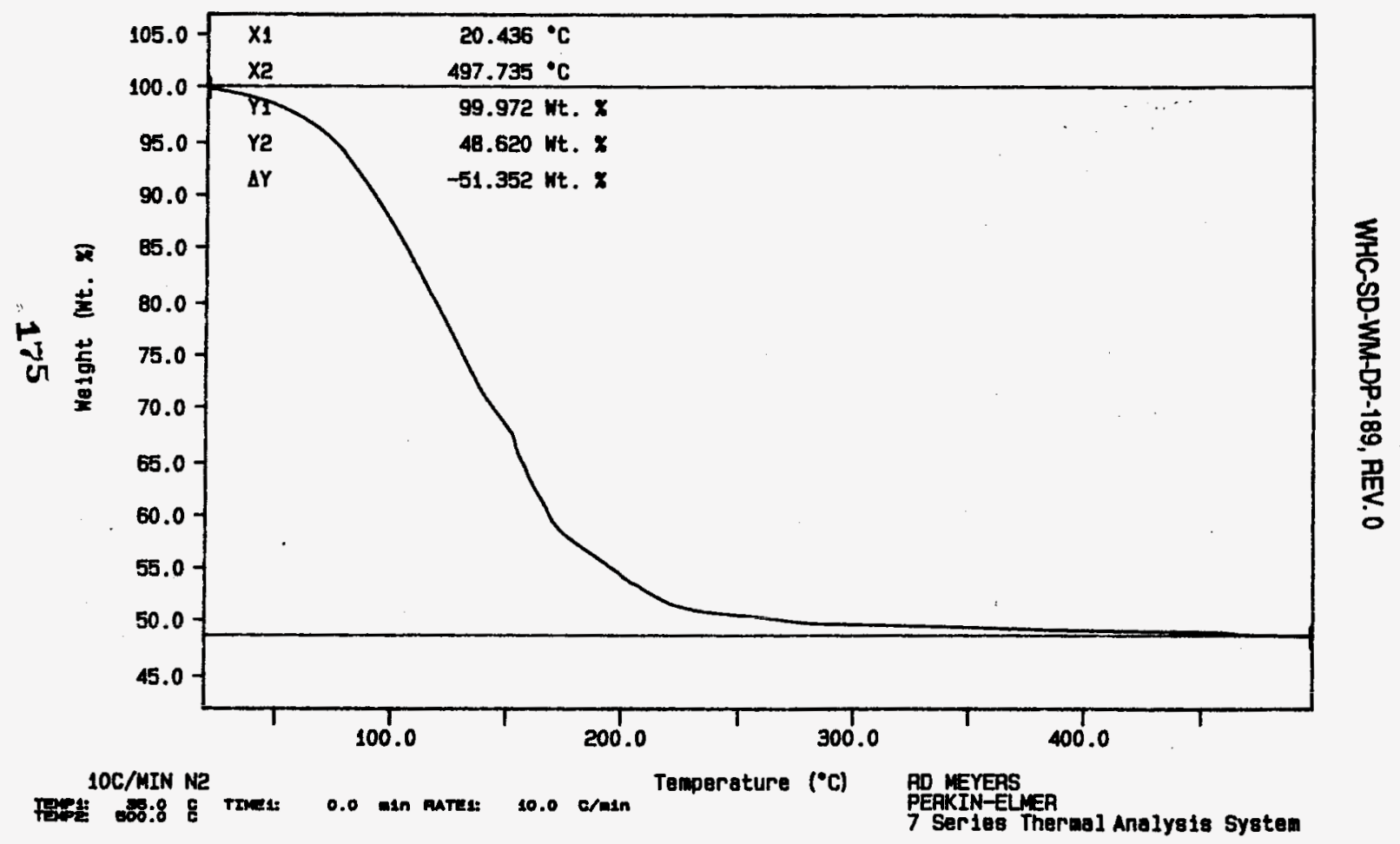




Analyst: YOD Instrument: TGAO I Book GLNBA

Method: LA-560-112 Rev/Mod B-I

Worklist Comment: U-102 TGA RUN UNDER N2. RCJ

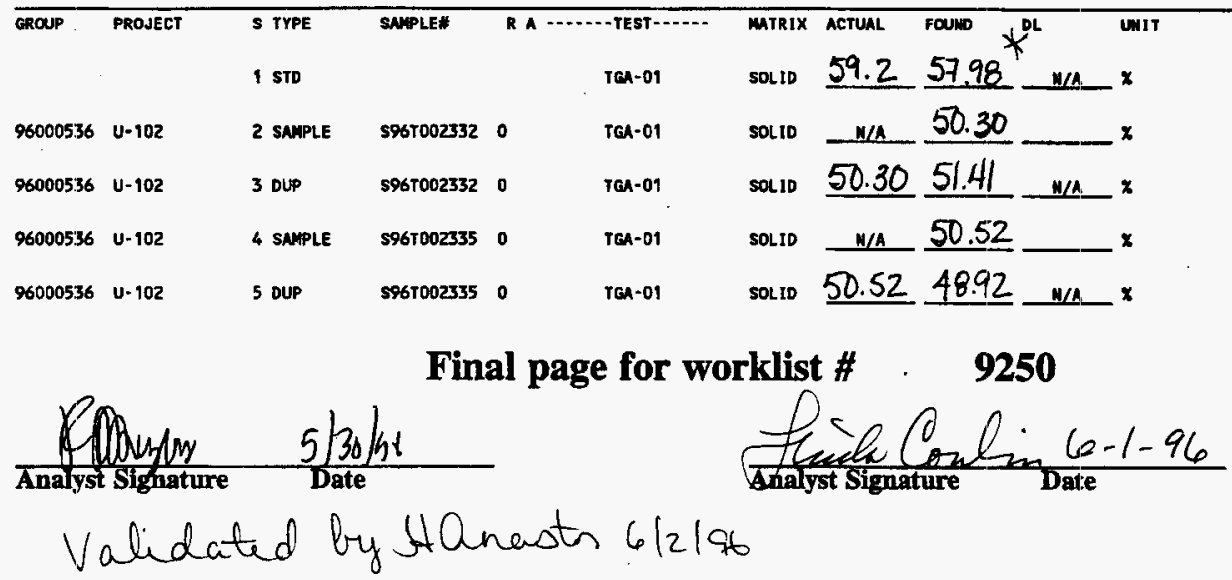

Data Entry Comments:

Units shown for QC (SPK \& STD) may not reflect the actual wits. $D L=$ Detection Limit, $S=$ Worklist Slot Number, $R=$ Replicate Number, $A=$ Aliquot Code. 
SIGNATURE BELOH REPRESENTS CHEMICAL TECHNOLOGIST/CHEMIST THAT

COMPLETED/VERIFIED THE CALIBRATION/ANALYSIS ON PAGES 177 TO 181.

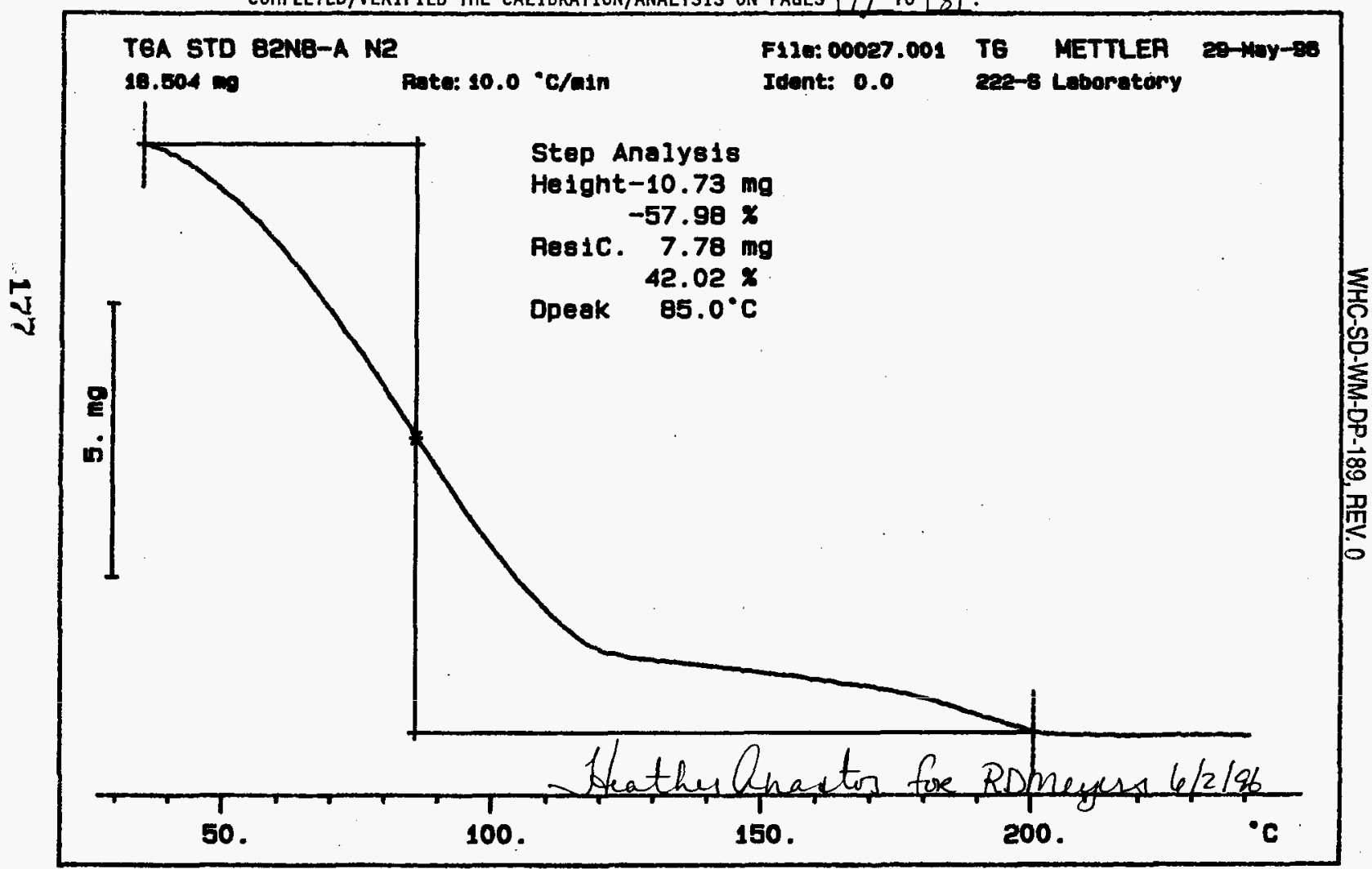




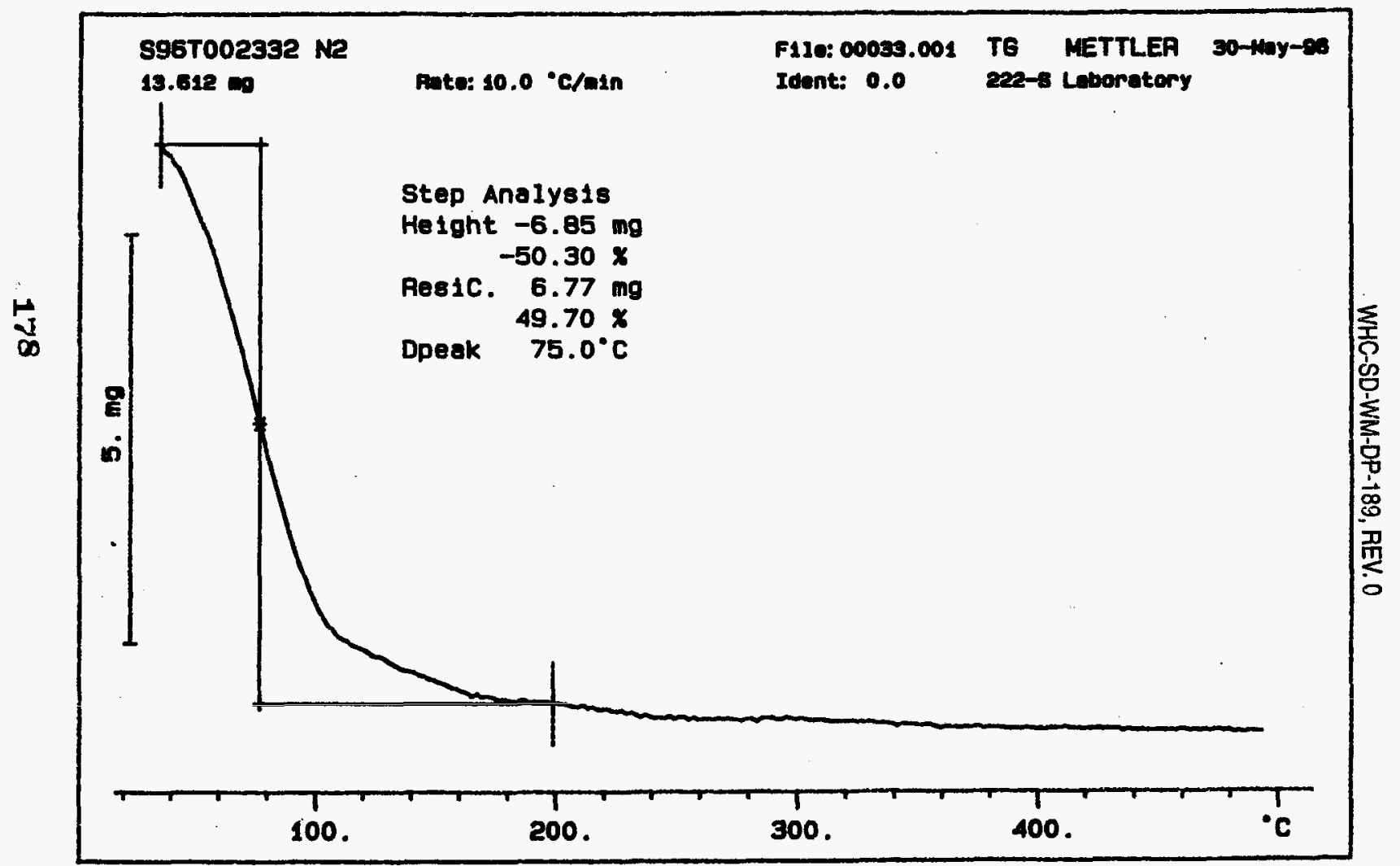




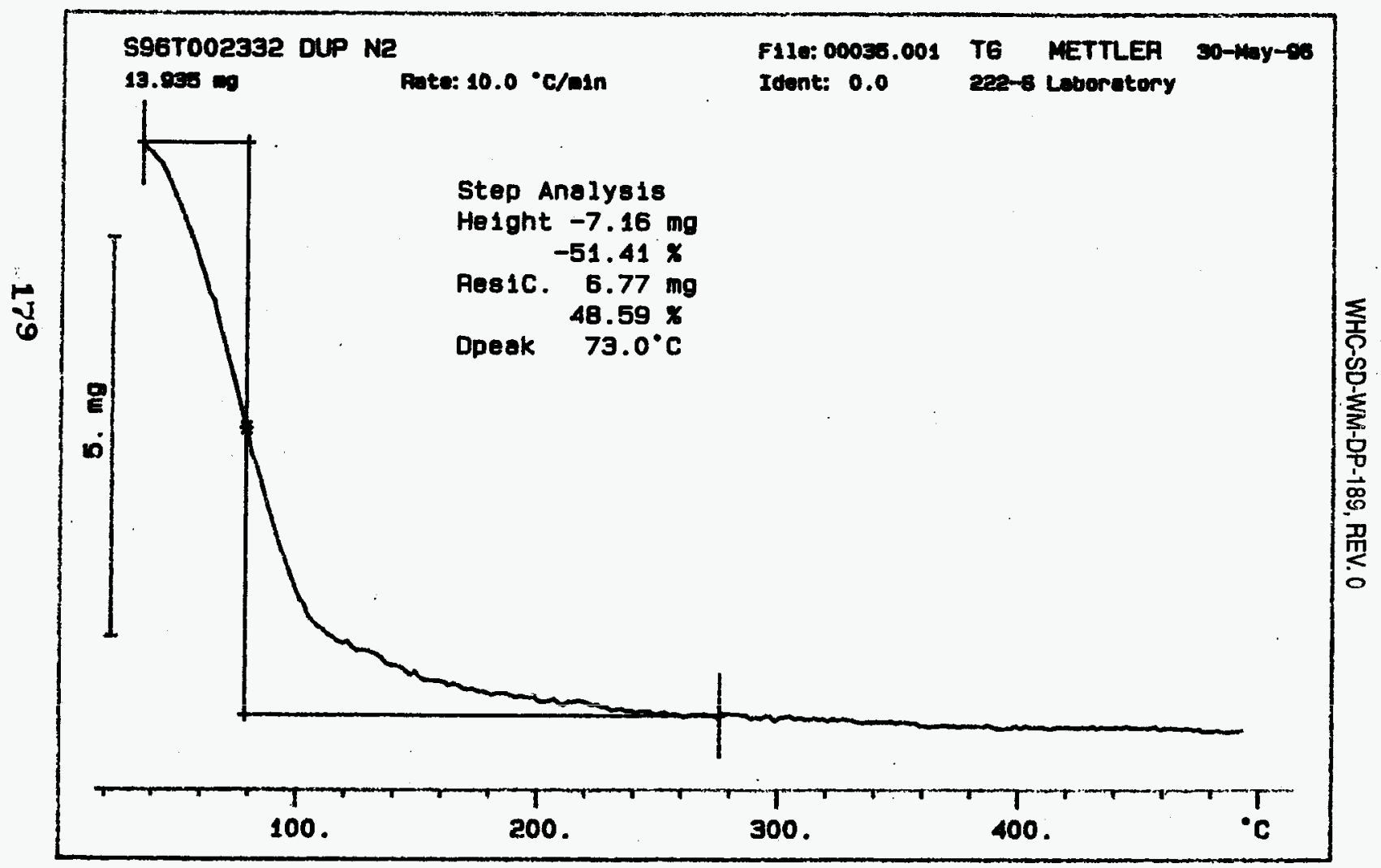




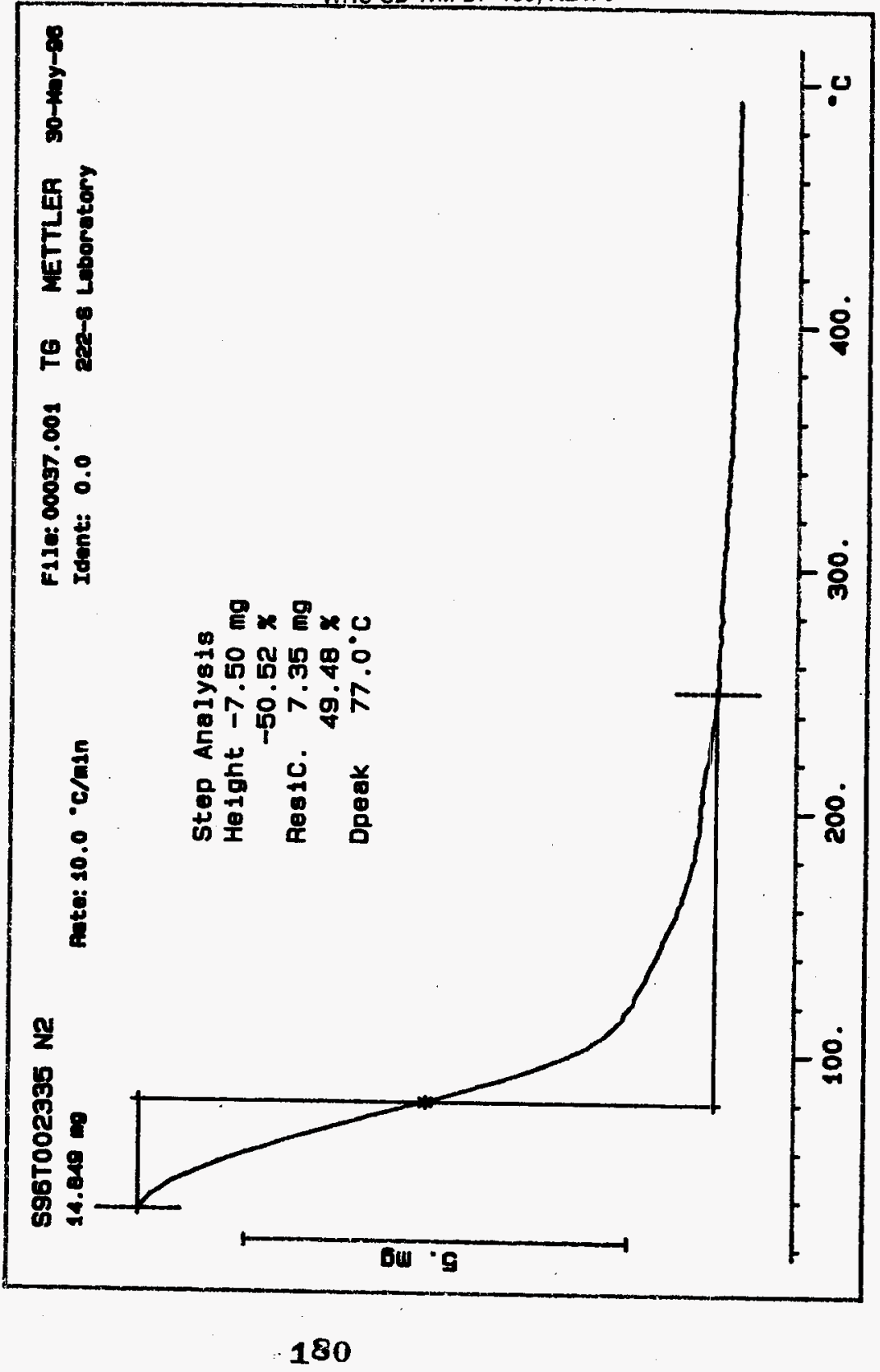




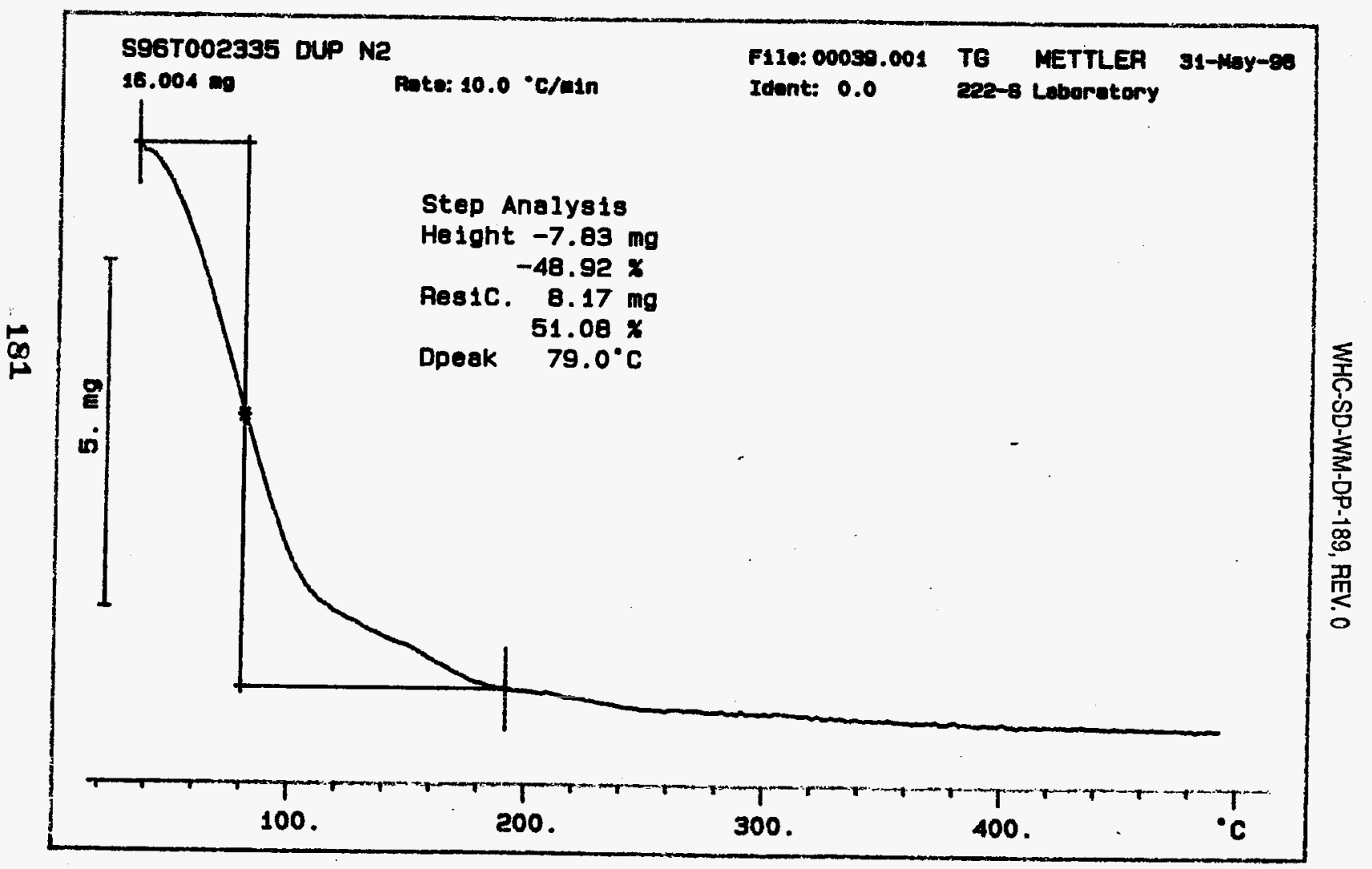


Analyst: $\quad$ KRM Instrument: TGAO I Book\# 82N8A

Method: LA-560-112 Rev/Mod B. I

Worklist Comment: U-102 TGA RUN UNDER N2. RCJ

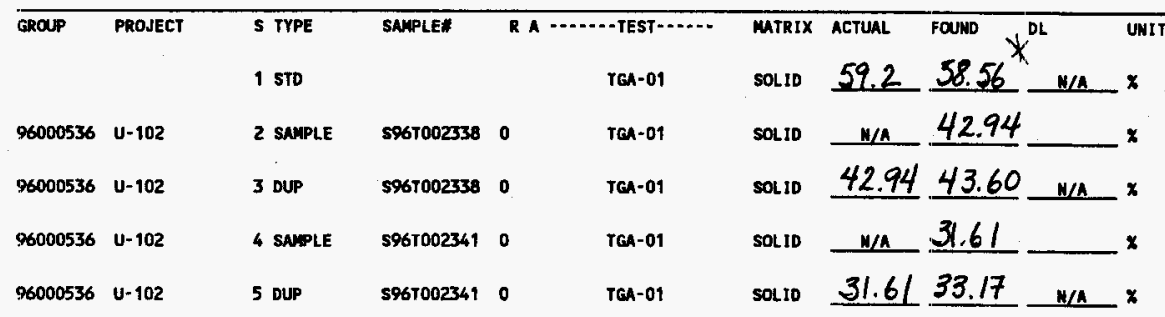

\section{$5-3146$ \\ Analyst Signature}

Validated by tha 6/4/96
9251

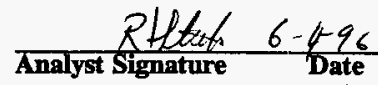

Data Entry Comments:

Units shown for QC (SPK \& STD) may not reflect the actual wits. $D L=$ Detection Limit, $S=$ Worklist Slot Number, $R=$ Replicate Number, $A=$ Aliquot Code. 
SIGNATURE BELOH REPRESENTS CHEMICAL TECHNOLOGIST/CHEMIST THAT

COMPLETED/VERIFIED THE CALIBRATION/ANALYSIS ON PAGES 183 TO 187.

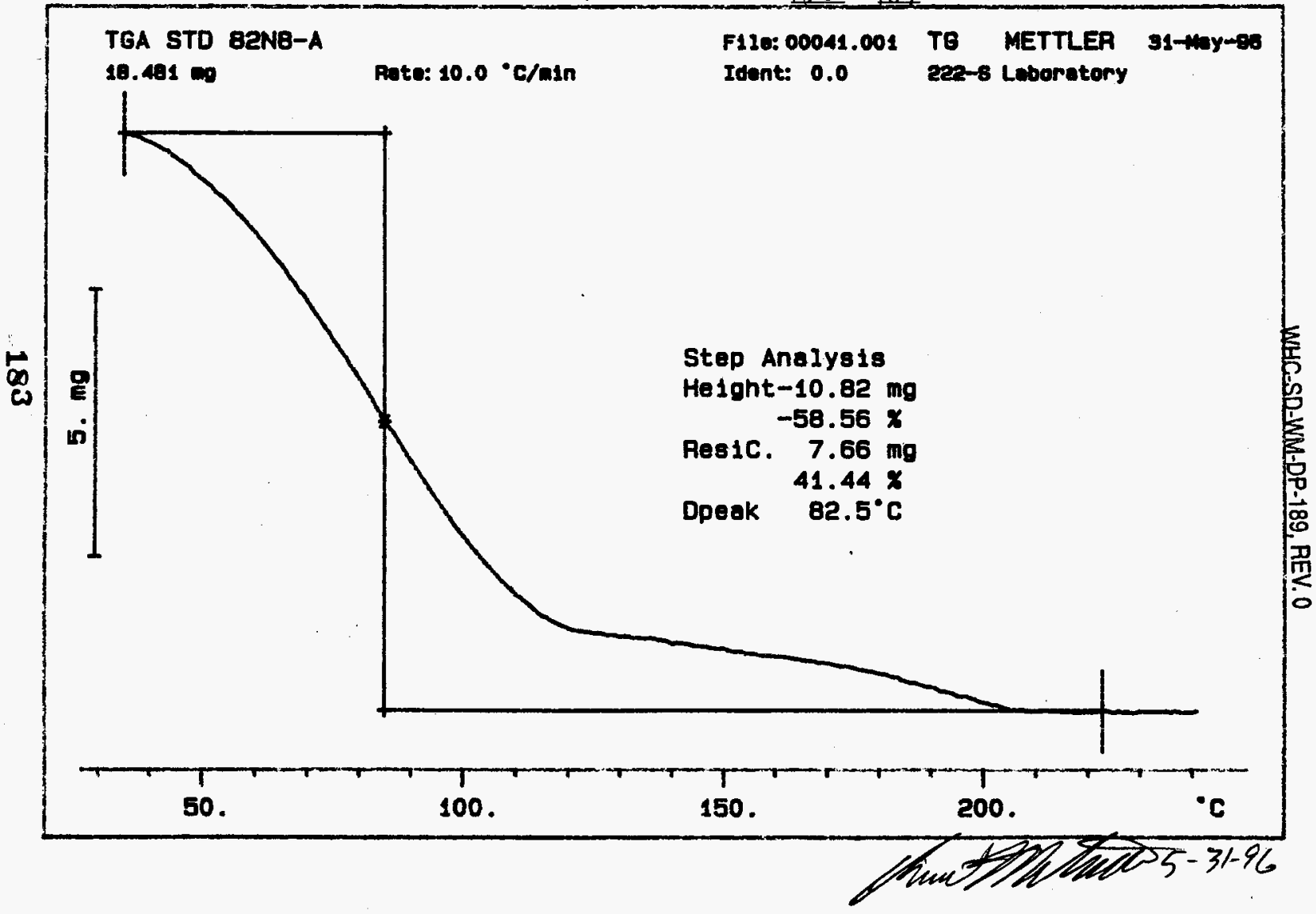




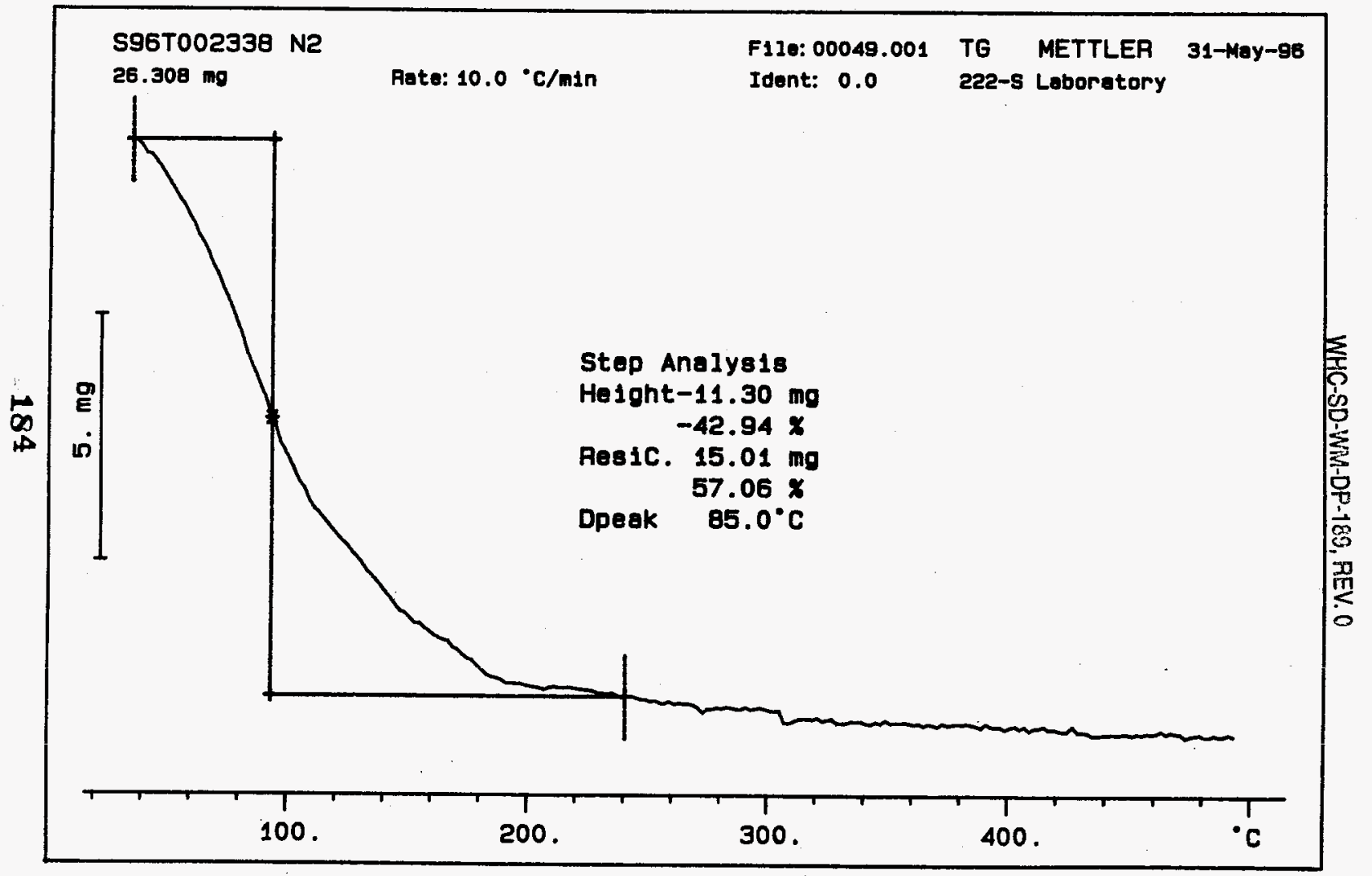




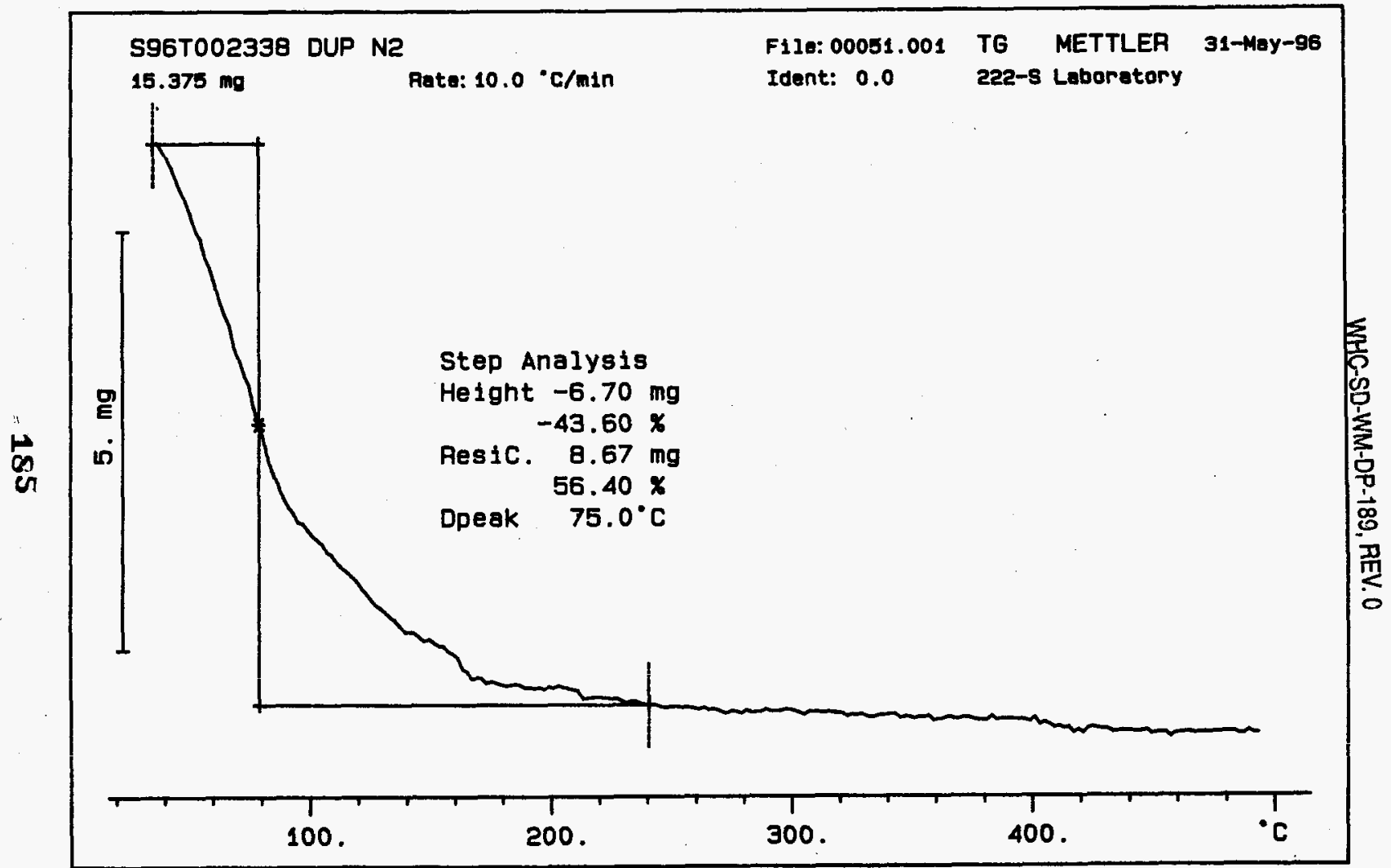




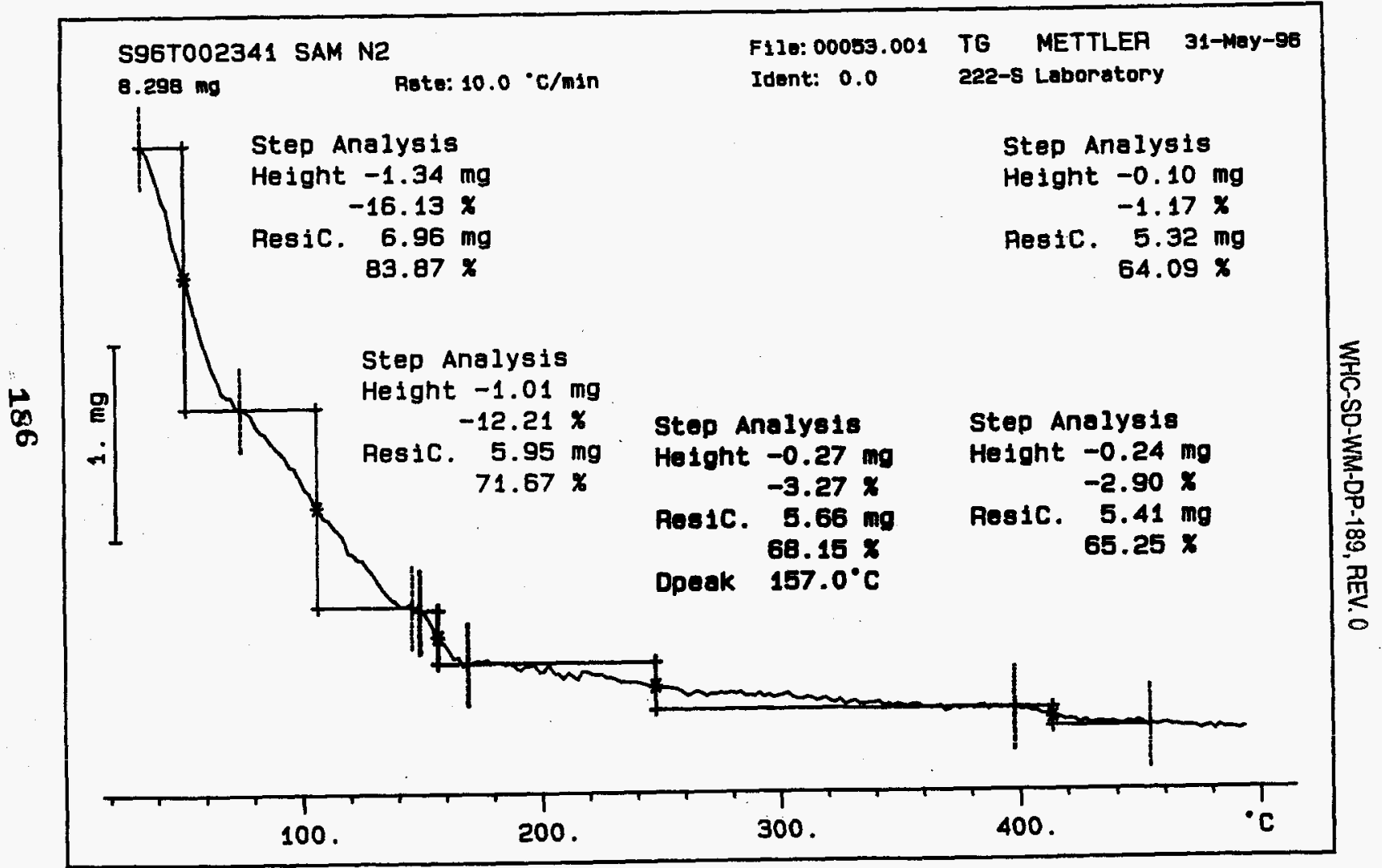




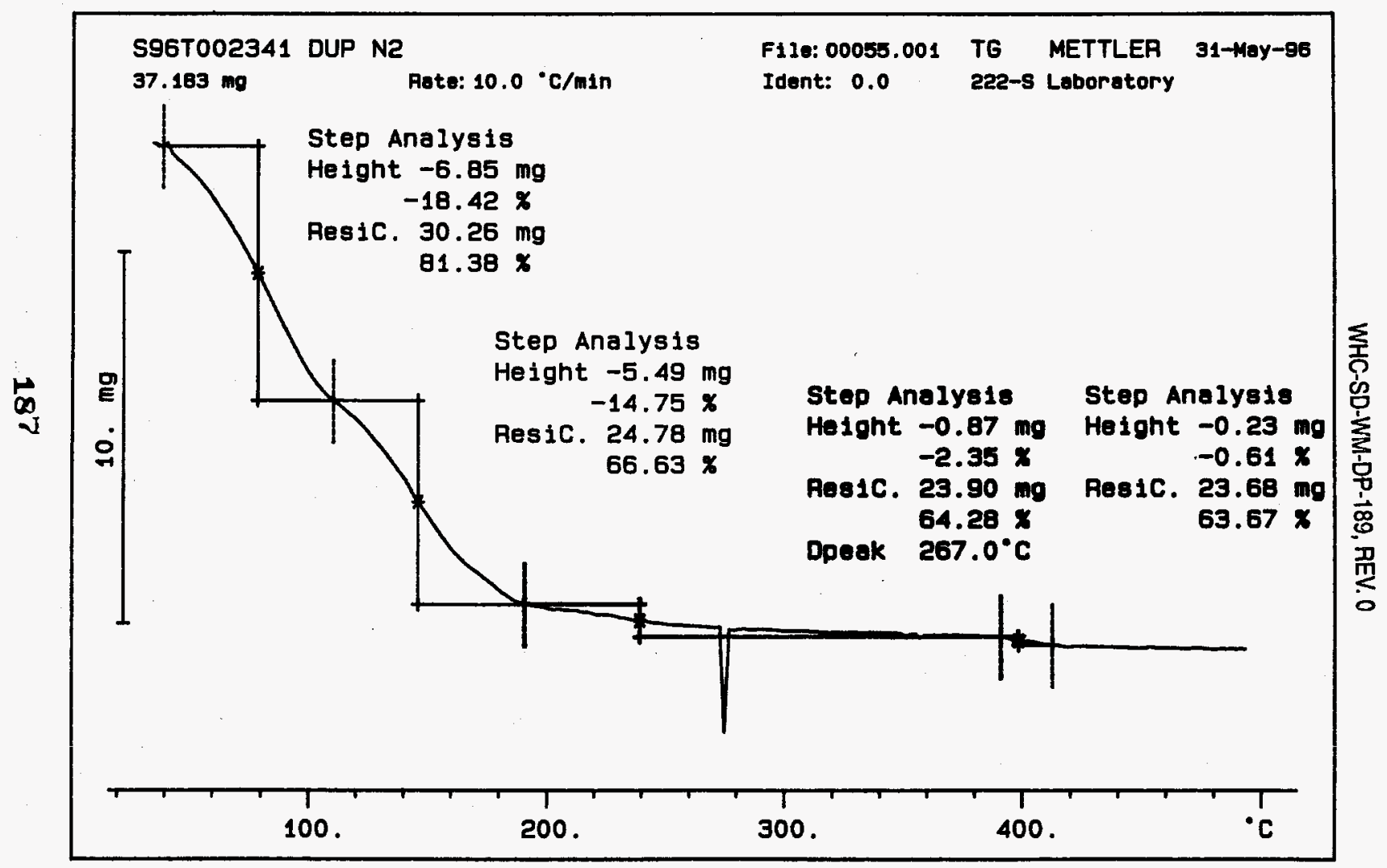




\section{LABCORE Data Entry Template for Worklist\#}

Analyst: $\quad$ ADP Instrument: TGAO $3 \quad$ Book \# 82 N8. A

Method: LA-514-114 Rev/Mod C-1

Worklist Comment: U-102 TGA RUN UNDER N2. RCJ

\begin{tabular}{|c|c|c|c|c|c|c|c|c|c|c|}
\hline GROAP & PROJECT & $\begin{array}{l}S \text { TYPE } \\
1 \text { STD }\end{array}$ & SAMPLE: & & $\begin{array}{l}\text {-TEST }-\cdots . .- \\
\text { TEA-03 }\end{array}$ & $\begin{array}{l}\text { MATRIX } \\
\text { SOLID }\end{array}$ & $\begin{array}{l}\text { Actual } \\
59.2 \\
\end{array}$ & $58.29^{\mathrm{DL}}$ & w/a & $\begin{array}{l}\text { Unit } \\
x\end{array}$ \\
\hline 96000536 & U-102 & 2 SNAPLE & \$96T002344 & 0 & TEA-03 & sol I0 & $N / A$ & 22.44 & & $x$ \\
\hline 96000536 & U-102 & 3 DUP $=$ & S96T002344 & 0 & זGA-03 & SOLID & 22.44 & 14.78 & . $/ \mathrm{A}$ & $x$ \\
\hline 96000536 & U-102 & 4 SAIPLE & 5967002347 & 0 & TGA-03 & SOL ID & $n / A$ & 46.82 & & $x$ \\
\hline 96000536 & $U-102$ & 5 DUP & S96T002347 & 0 & $\tan -03$ & sol to & 46.82 & 43.20 & $w / \mathbf{A}$ & $x$ \\
\hline
\end{tabular}

\section{Final page for worklist \# $\quad 9252$}

Sie attached for signatures

Analyst Signature Date 6/3196

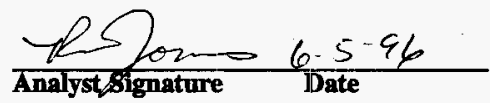

Validated by Hanasto $6-6-96$

S96700 2344 bill be remen because of the Shape of
the thermogram being 80 different and the high RPD.

Data Entry Comments:

Units shown for QC (SPK \& STD) may not neflect the actual wits. $D L=$ Detection Limit, $S=$ Worklist Slot Number, $R=$ Replicate Number, $A=$ Aliquot Code. 


\section{LABCORE Data Entry Template for Worklist\#"}

Analyst: ADD Instrument: TGA0 Book\# $82 \mathrm{~N} 8 \mathrm{~A}$

Method: LA-560-112 Rev/Mod $\mathrm{C}-1$

Worklist Comment: U-102 TGA RUN UNDER N2. RCJ

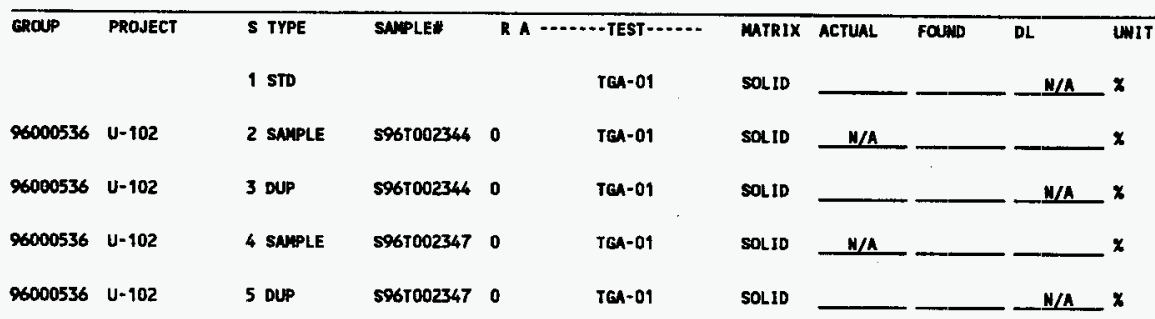

\section{Final page for worklist \# $\quad 9252$}

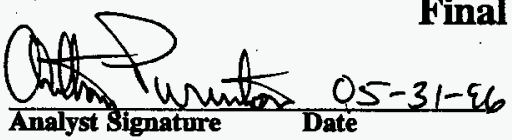

\section{Analyst Signature Date}

Data Bntry Comments:

Units shown for QC (SPK \& STD) may not reflect the actual units. $D L=$ Detection Limit, $S=$ Worklist Slot Number, $R=$ Replicate Number, $A=A l i q u o t$ Code. 
Curve 1: TGA

F1le Info: TER053101 Fr1 May 31 15: 36: 181996

Sanple Weight: 14.833 ang

TGA STD BaNB-A

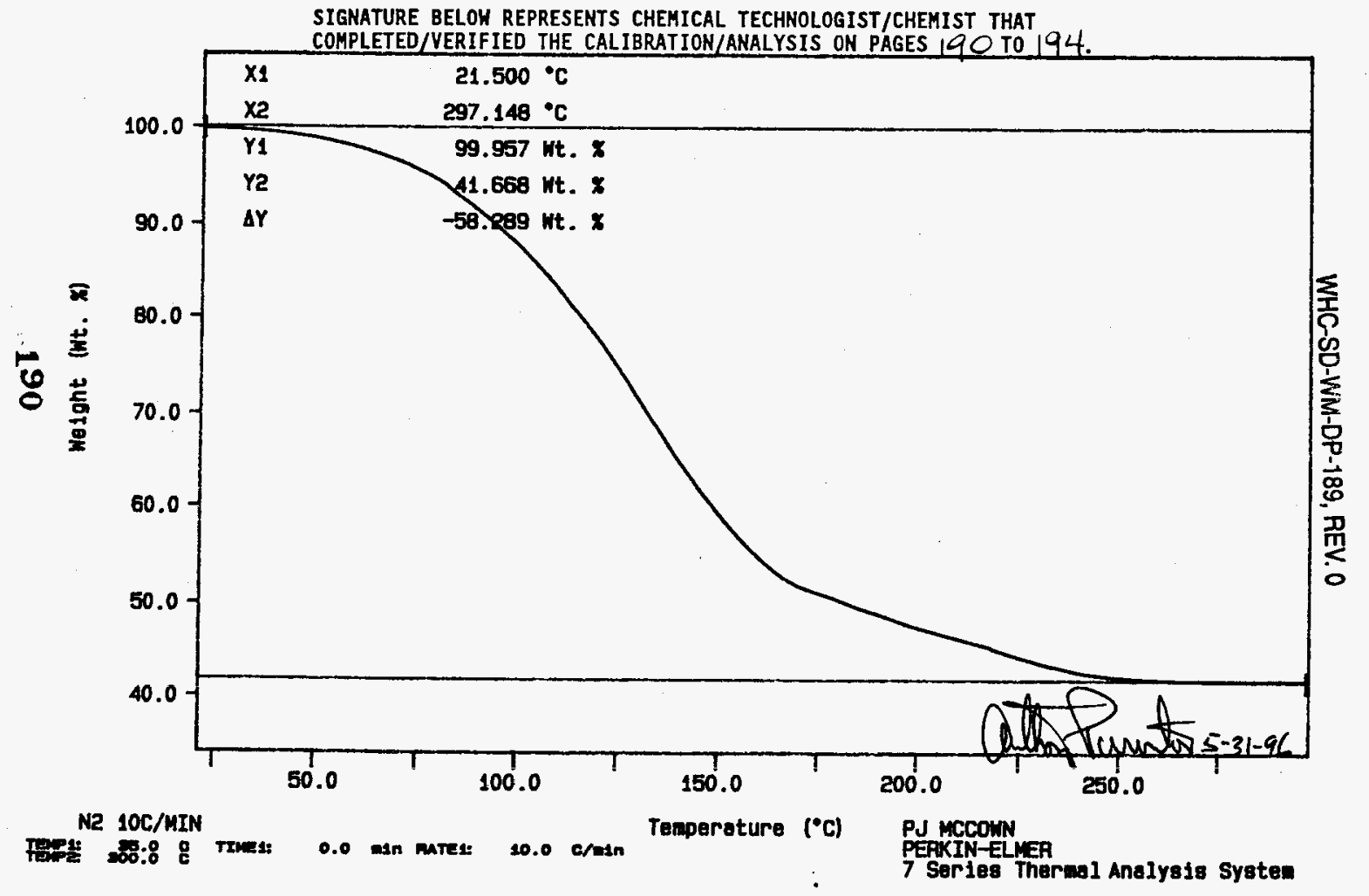


Curve 1: TGA

F1le info: SAM053106 Sat Jun 1 02: $55: 021996$

Sanple Weight: 29.404

S96T002344 SAM

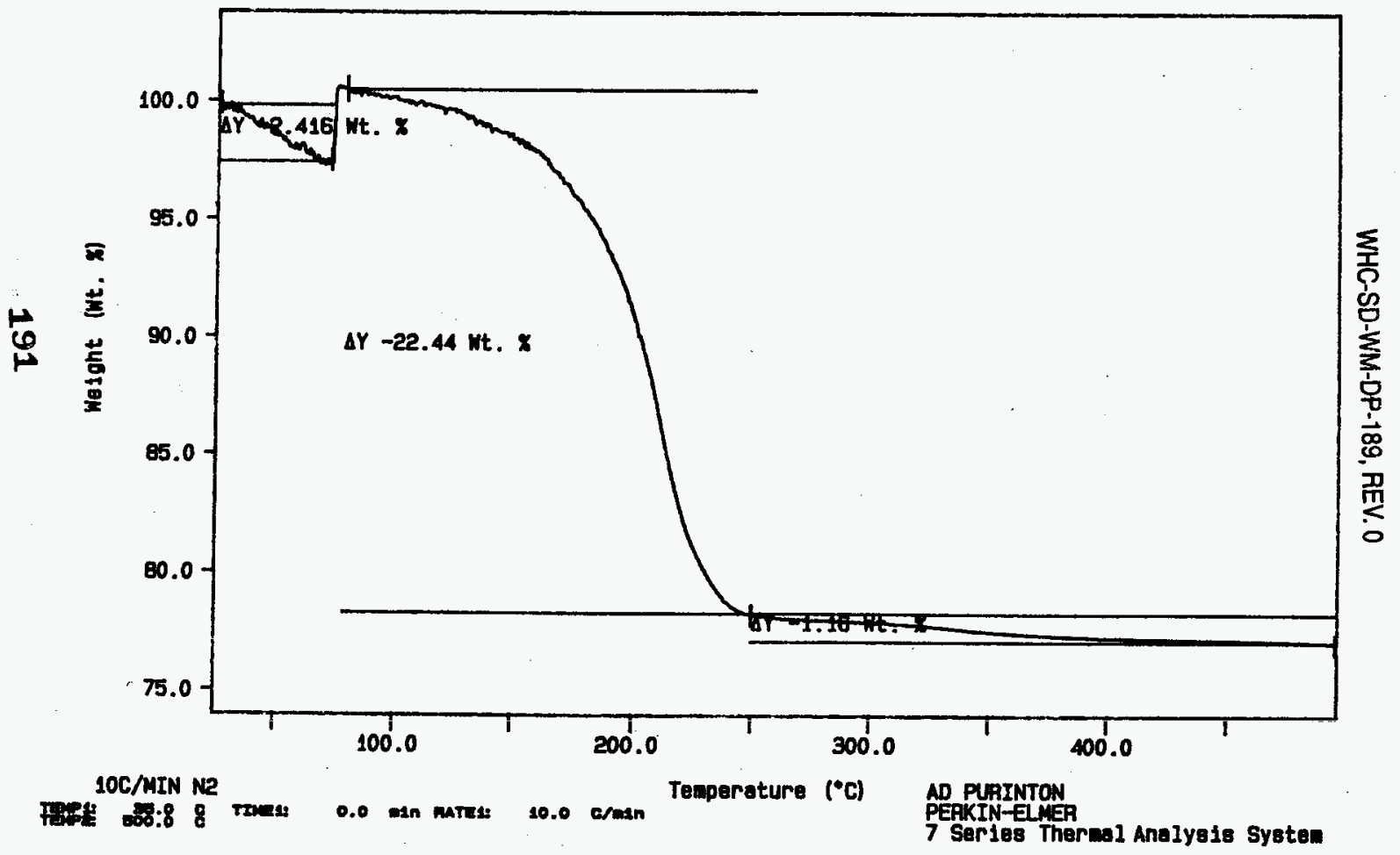


Curve 1: TGA

F1le info: SAM053107 Sat Jun 1 04: 06: 301996

Sample Neight: 29.141 ing

S96T002344 EAH 150

Dup $5-3196$

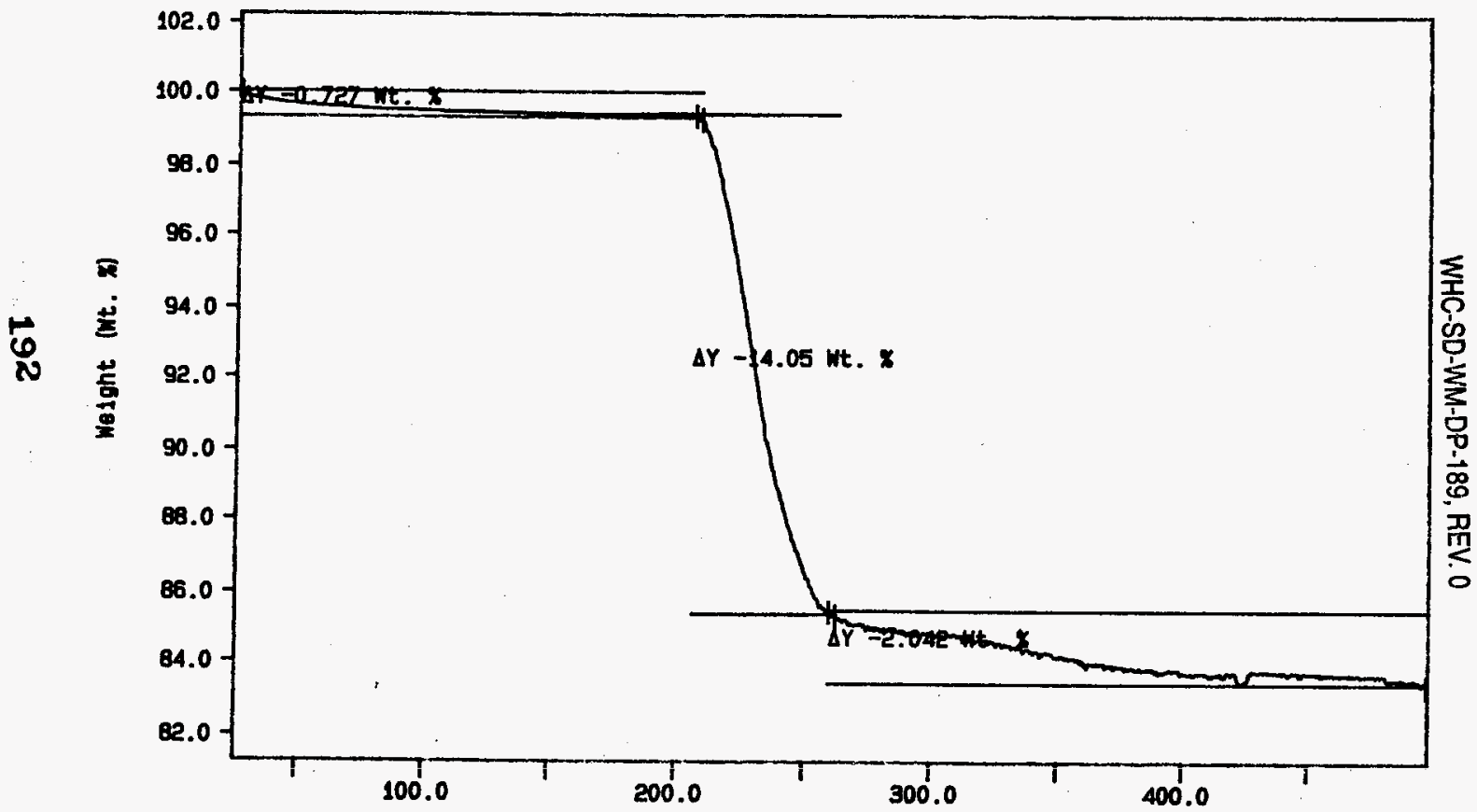

10C/MIN NP

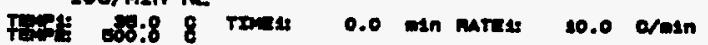

Teaperature ("C) AD PUAINTON

PERKIN-ELLER

7 Ser 108 Thermal Analysis System 
Curve 1: TGA

File Info: SAM053103 Fr1 May 31 23: Z5: 161996

Sample Neight: 21.545

S96T002347 SAM

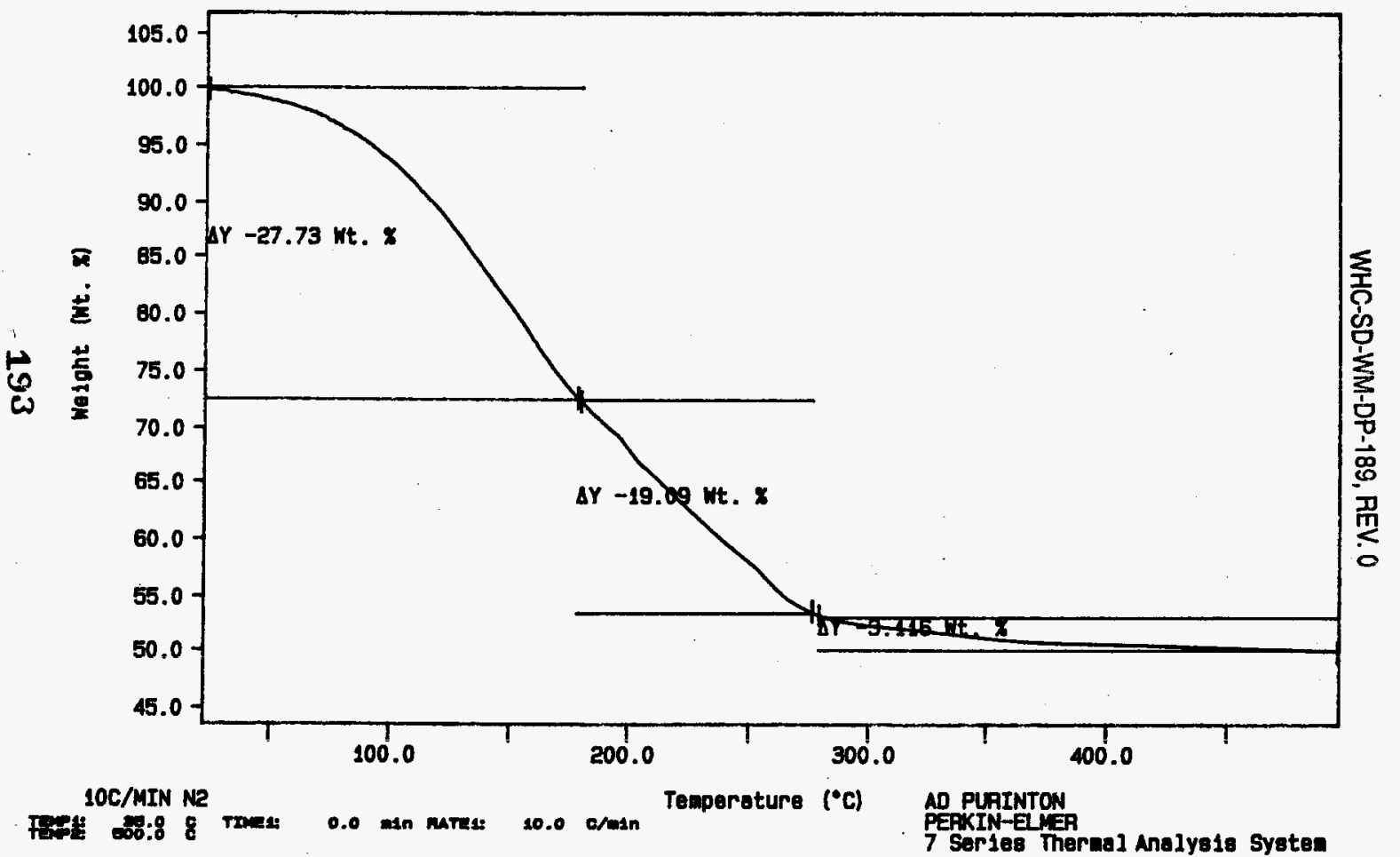


Curve 1: TGA

F1le info: SAM059104 Sat Jun 101: 15: 28 1996

Sanple We1ght: $23.128 \mathrm{mg}$

\$96T002347 DUP

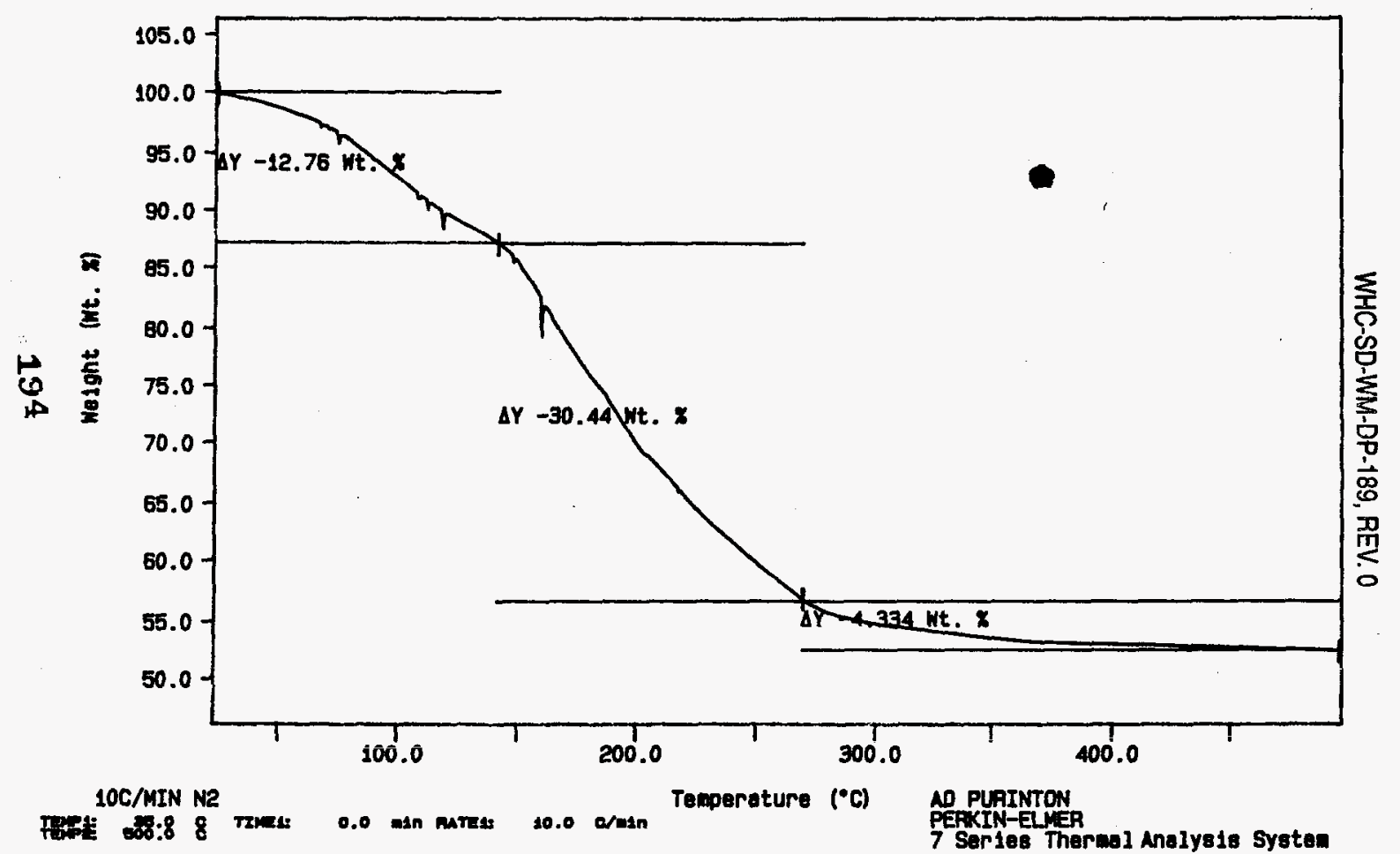




\section{LABCORE Data Entry Template for Worklist;"}

Analyst: ADP Instrument: TGAO $\perp$ Book \# \$2NSA

Method: LA-560-112 Rev/Mod B-1

Worklist Comment: U-102 TGA RUN UNDER N2. RCJ

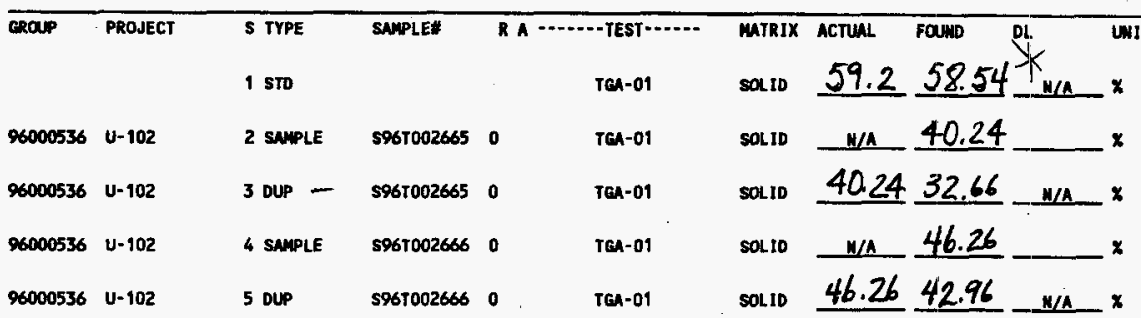

\section{Final page for worklist \# 9383}

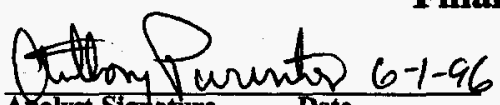

Ailalyst Signature Date

Validated by Sthnaoton b-6.96

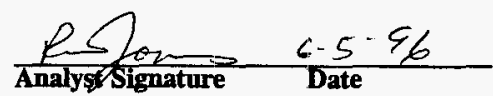

1

Data Bnary Comments: 9967002665 duplicate reaults are the aum of two wright loos steps.

Units shown for QC (SPK \& STD) may not reflect the actual units. $D L=$ Detection Limit, $S=$ Worklist Slot Niumber, $R=$ Replicate Number, $A=$ Aliquot Code. 


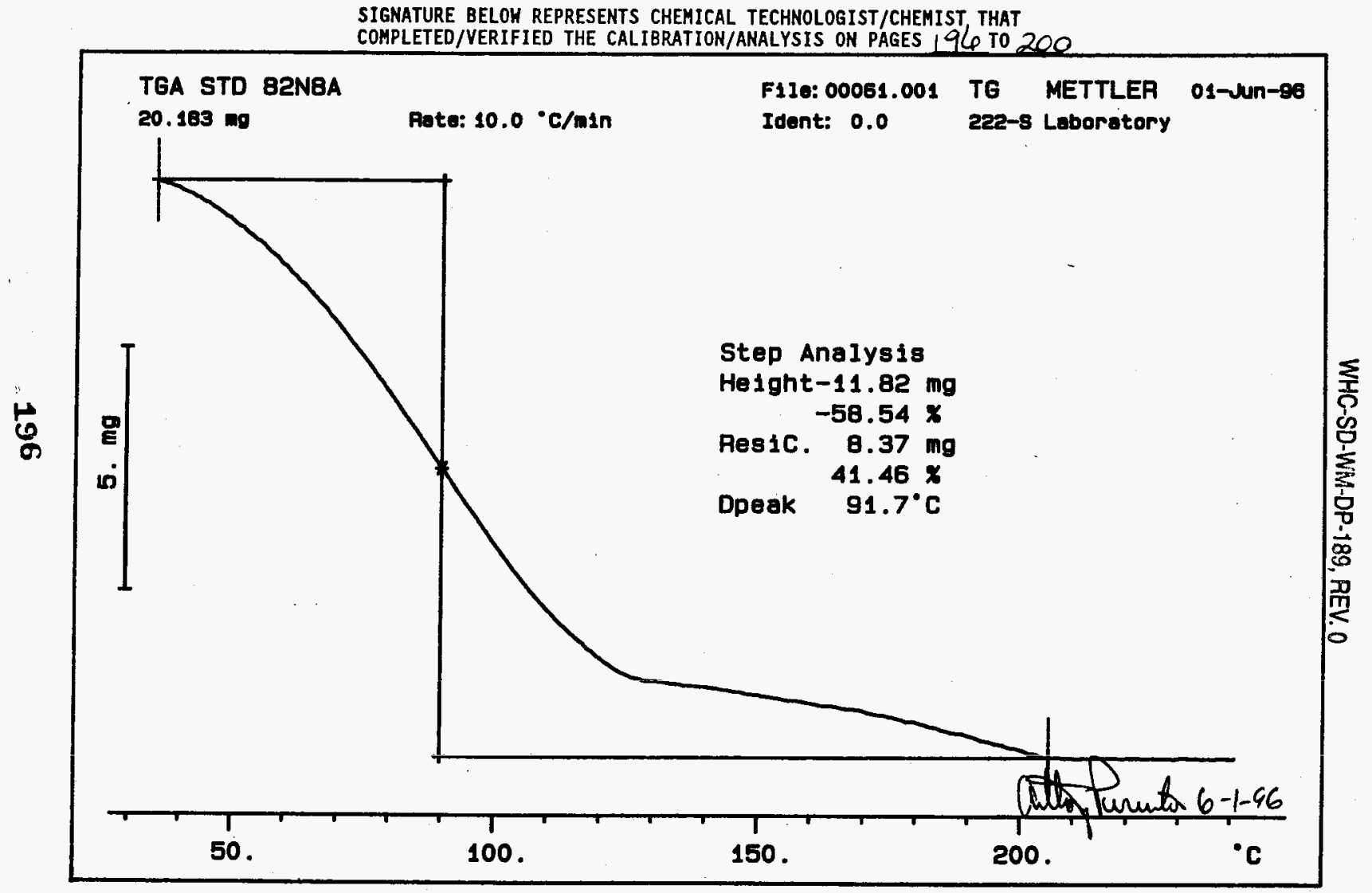




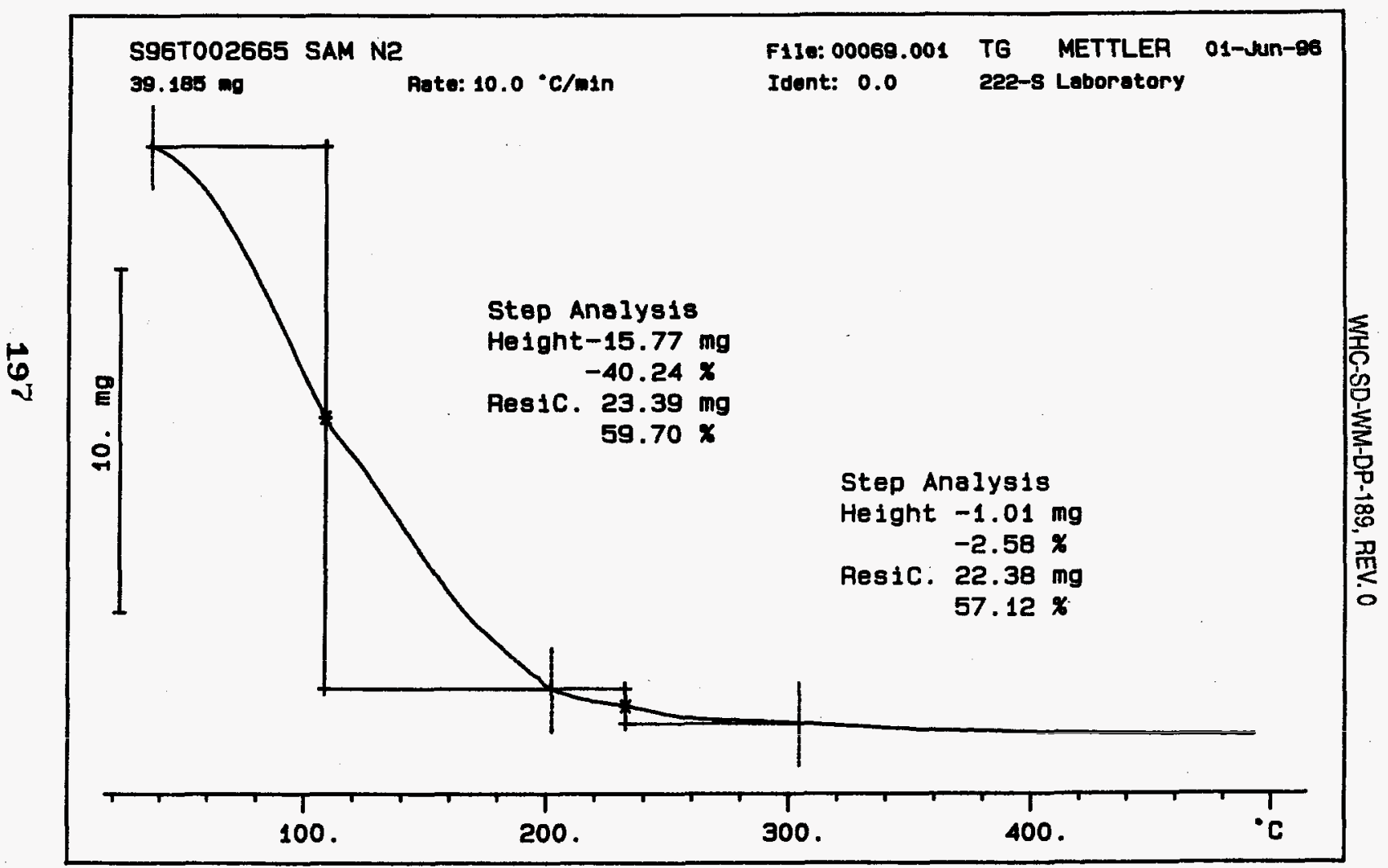




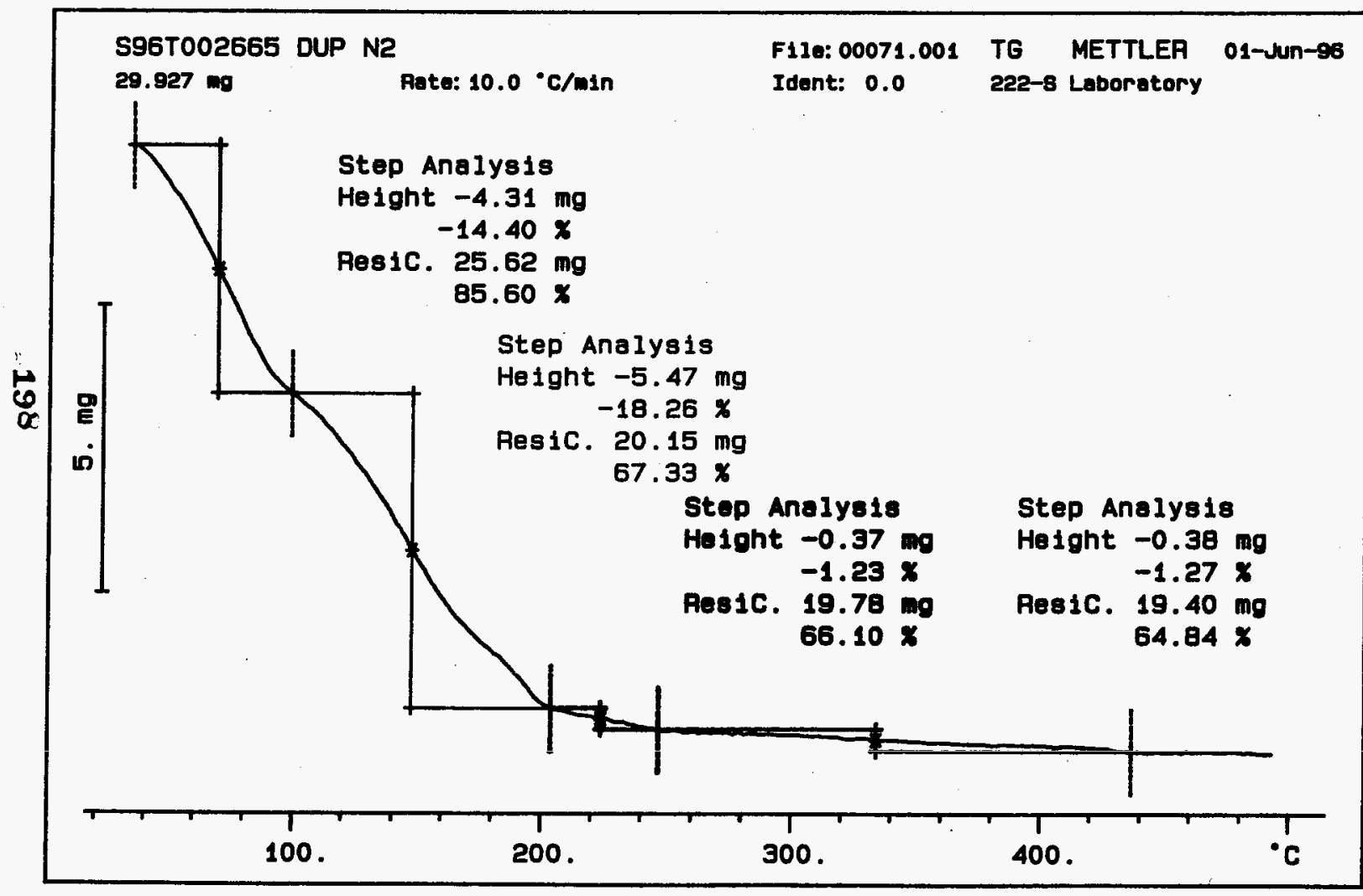




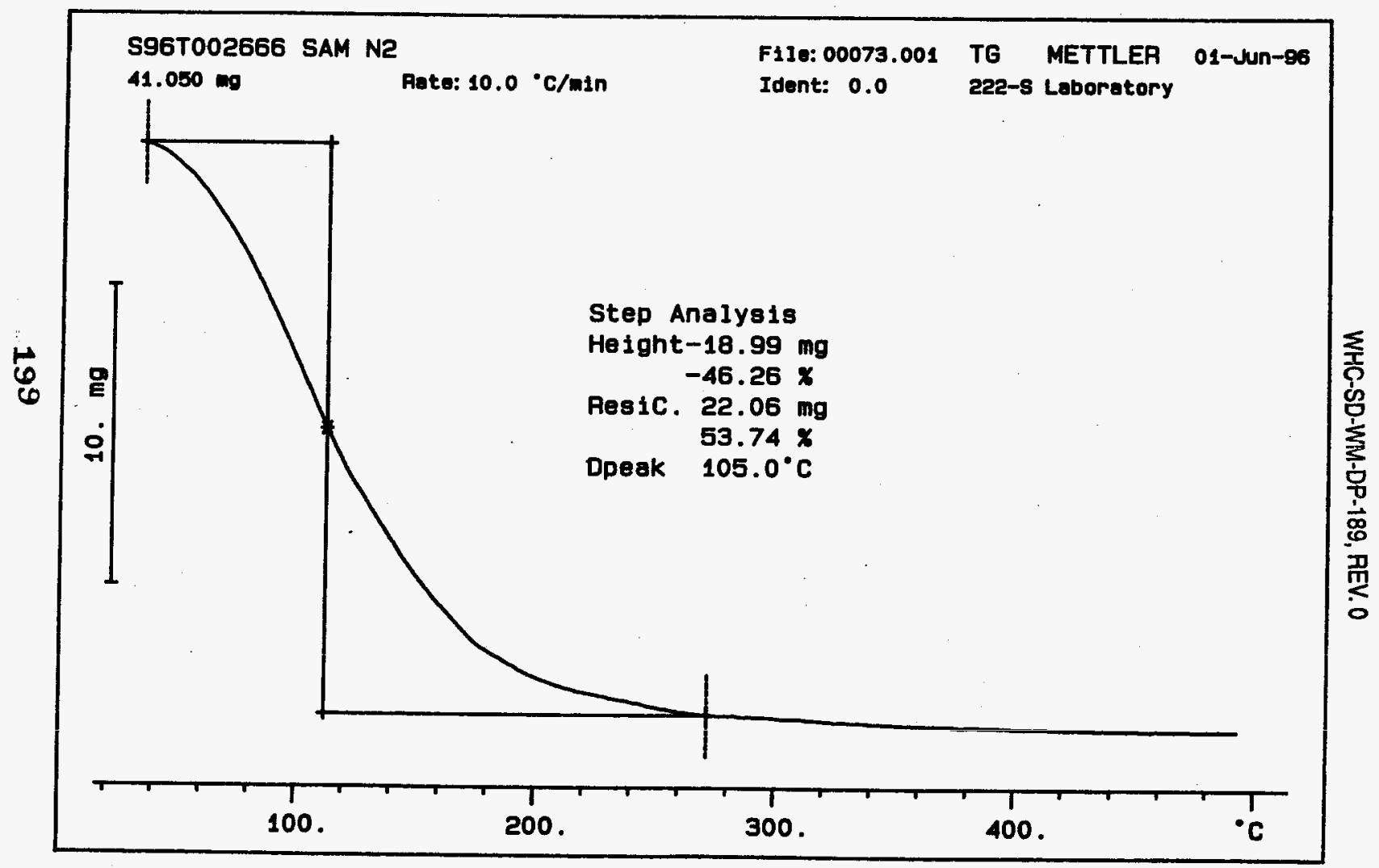




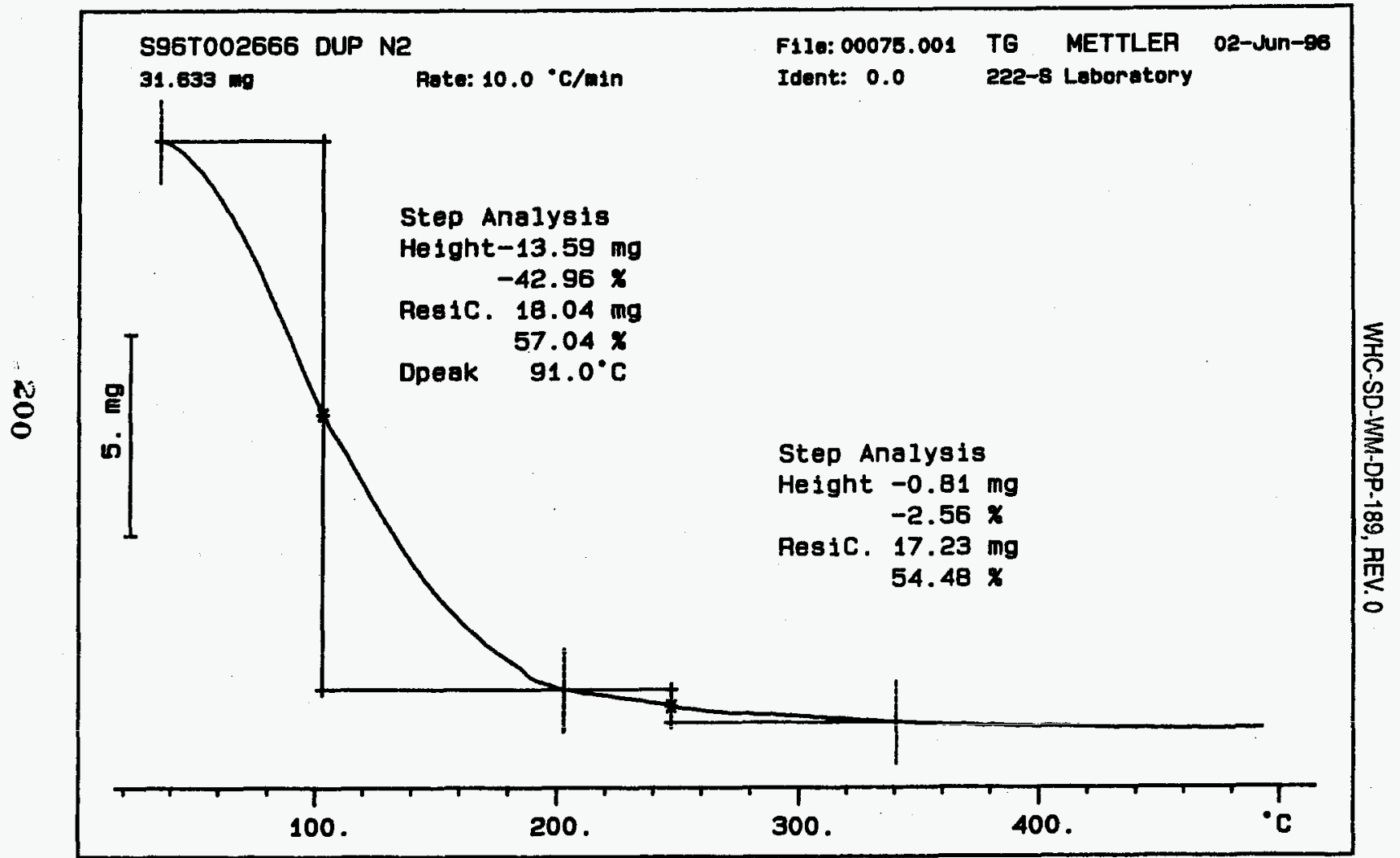




\section{LABCORE Data Entry Template for Worklist\#}

\begin{tabular}{|c|c|c|c|}
\hline Analyst: & AD & Instrument: TGA0 & Book \# $\$ 2 N 18 A$ \\
\hline
\end{tabular}

Worklist Comment: U-102 TGA RUN UNDER N2. RCJ

\begin{tabular}{|c|c|c|c|c|c|c|c|c|c|c|}
\hline GROUP & PROJECT & $\begin{array}{l}\text { S TYPE } \\
1 \text { STD }\end{array}$ & SAMPLE* & & $\begin{array}{l}\text { TEST- } \\
\text { TEA-01 }\end{array}$ & $\begin{array}{l}\text { MATRIX } \\
\text { SOLID }\end{array}$ & $\begin{array}{l}\text { ACTUAL } \\
59.2 \\
\end{array}$ & $\begin{array}{l}58.42 \\
\end{array}$ & $w / A$ & $\begin{array}{l}\text { UNIT } \\
x\end{array}$ \\
\hline 96000536 & $U-102$ & 2 SMMPLE & 5967002755 & 0 & TGA-0I & SOLID & n/a & 41.68 & & $x$ \\
\hline 96000536 & U-102 & 3 DUP & S967002755 & 0 & TEA-01 & SOLID & 41.68 & 40.7 & $N / A$ & $x$ \\
\hline
\end{tabular}

Final page for worklist \#

9384

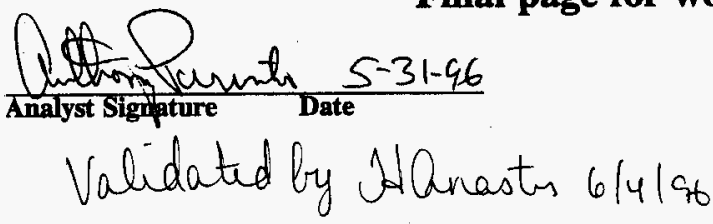

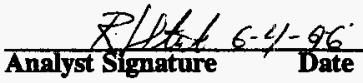

$$
\text { Validated by Nanasts 6/4/at }
$$

Data Entry Comments:

Units shown for $Q C$ (SPK \& STD) may not reflect the actual units. $D L=$ Detection Limit, $S=$ Worklist Slot Number, $R=$ Replicate Number, $A=$ Aliquot Code. 


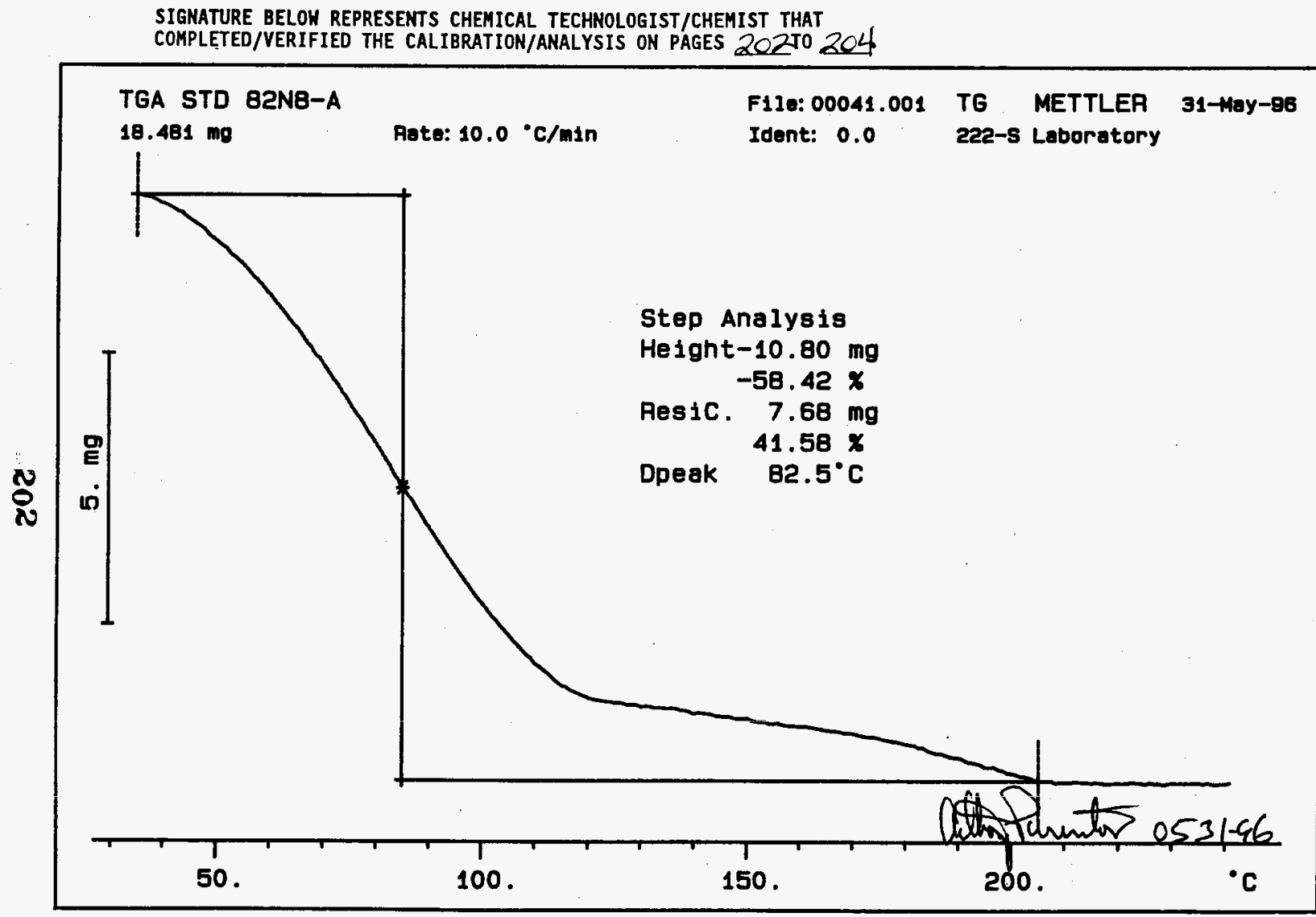




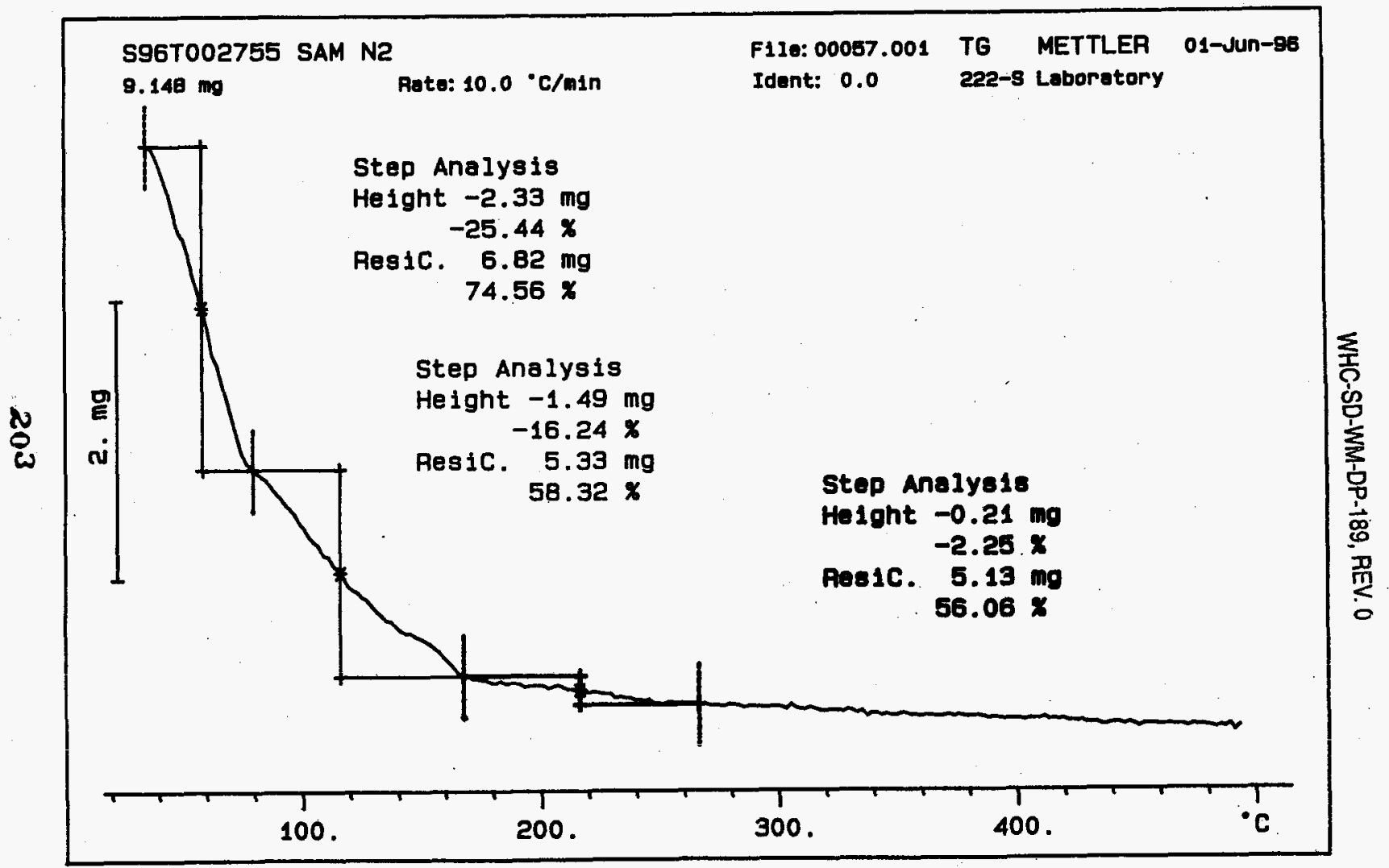




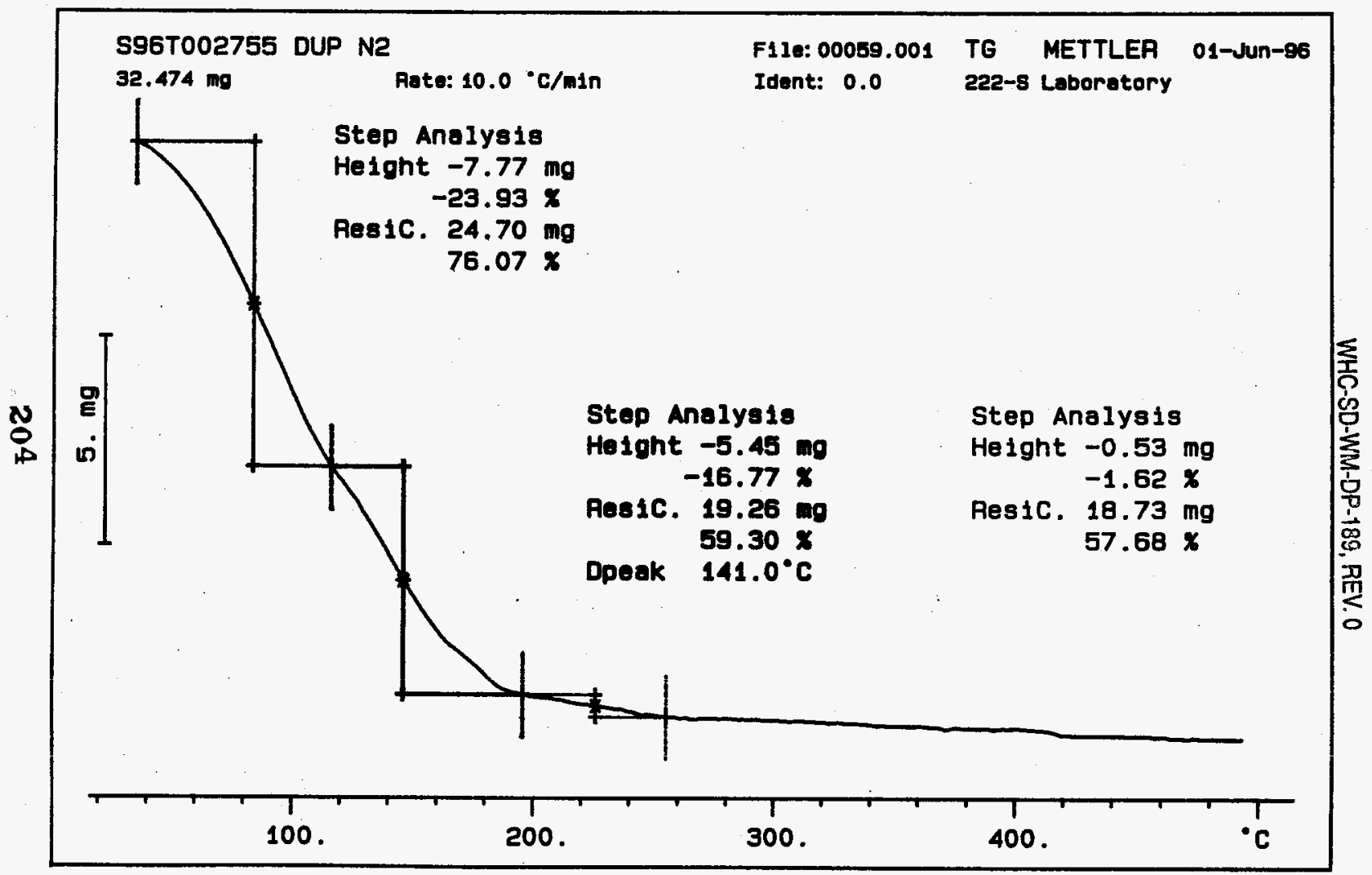


Analyst: SMF Instrument: TGA0 I Book\#82118 Method: LA-560-112 Rev/Mod B-1

Worklist Comment: U-102 TGA RUN UNDER N2. RCJ

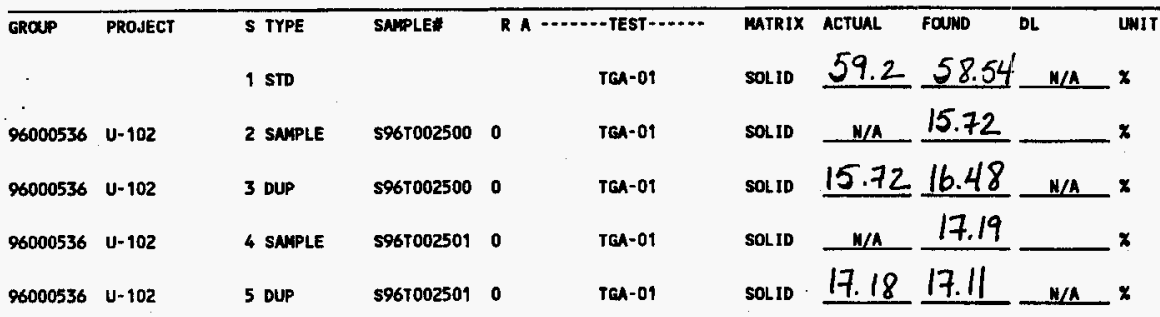

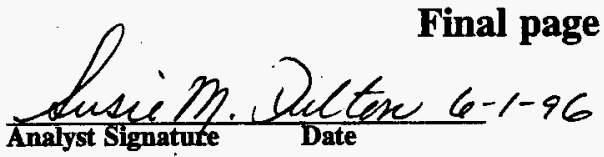

Validated by $1 /$ anastos $6 / 4 / 96$

Data Entry Comments:

Units shown for $Q C$ (SPK \& STD) may not reflect the actual units. $D L=$ Detection Limit, $S=$ Worklist Slot Number, $R=$ Replicate Number, $A=$ Aliquot Code. 


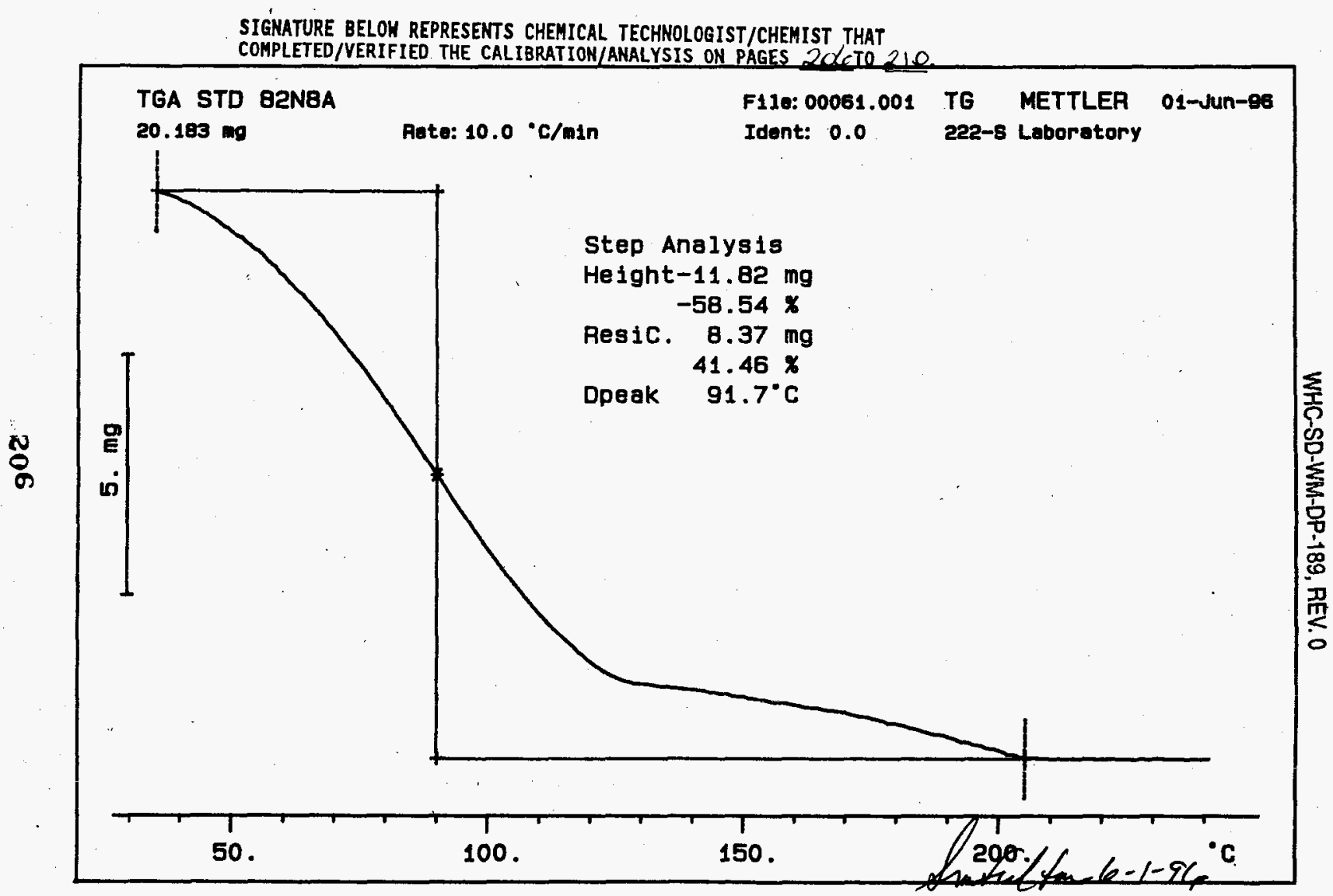




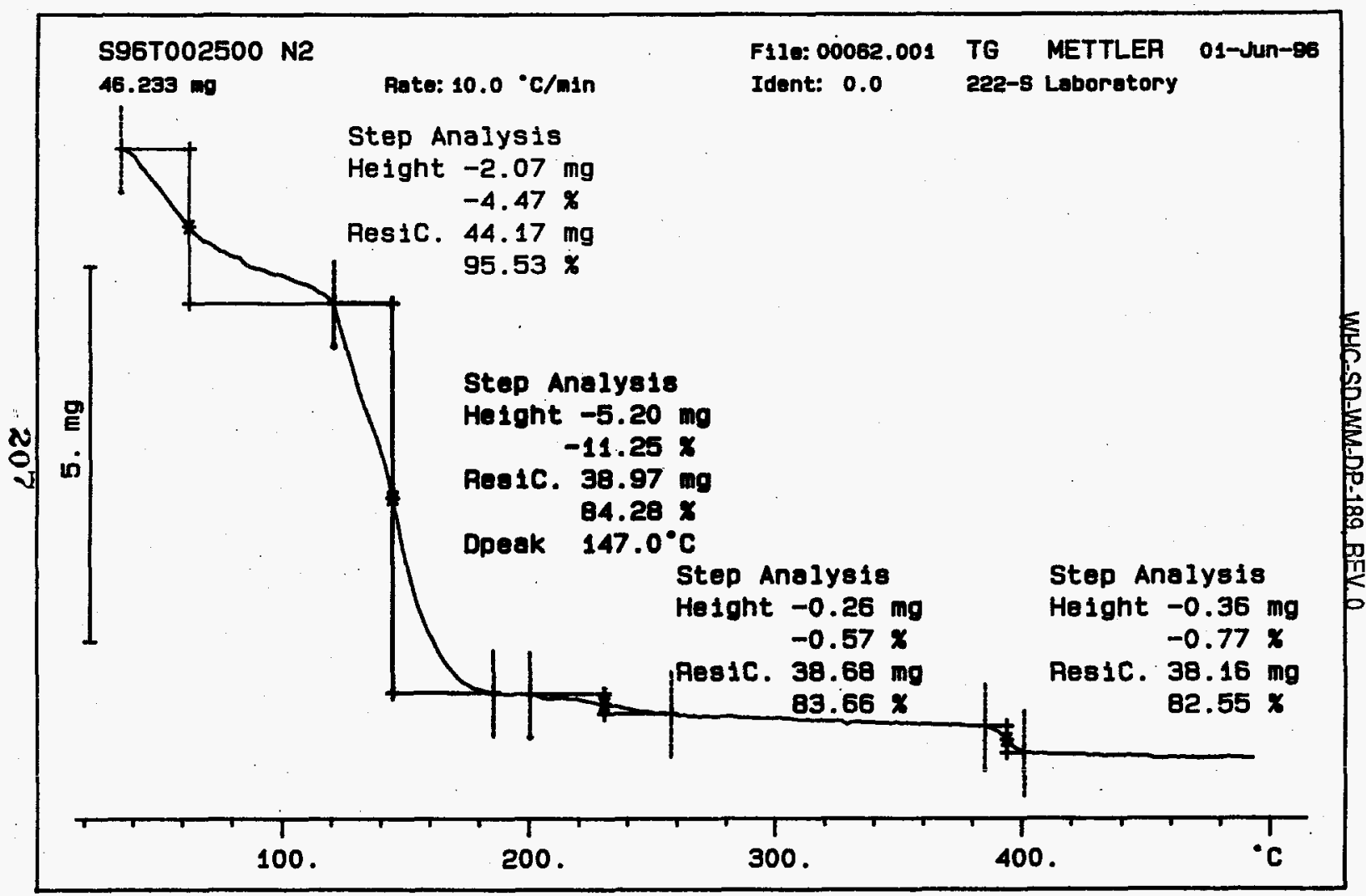




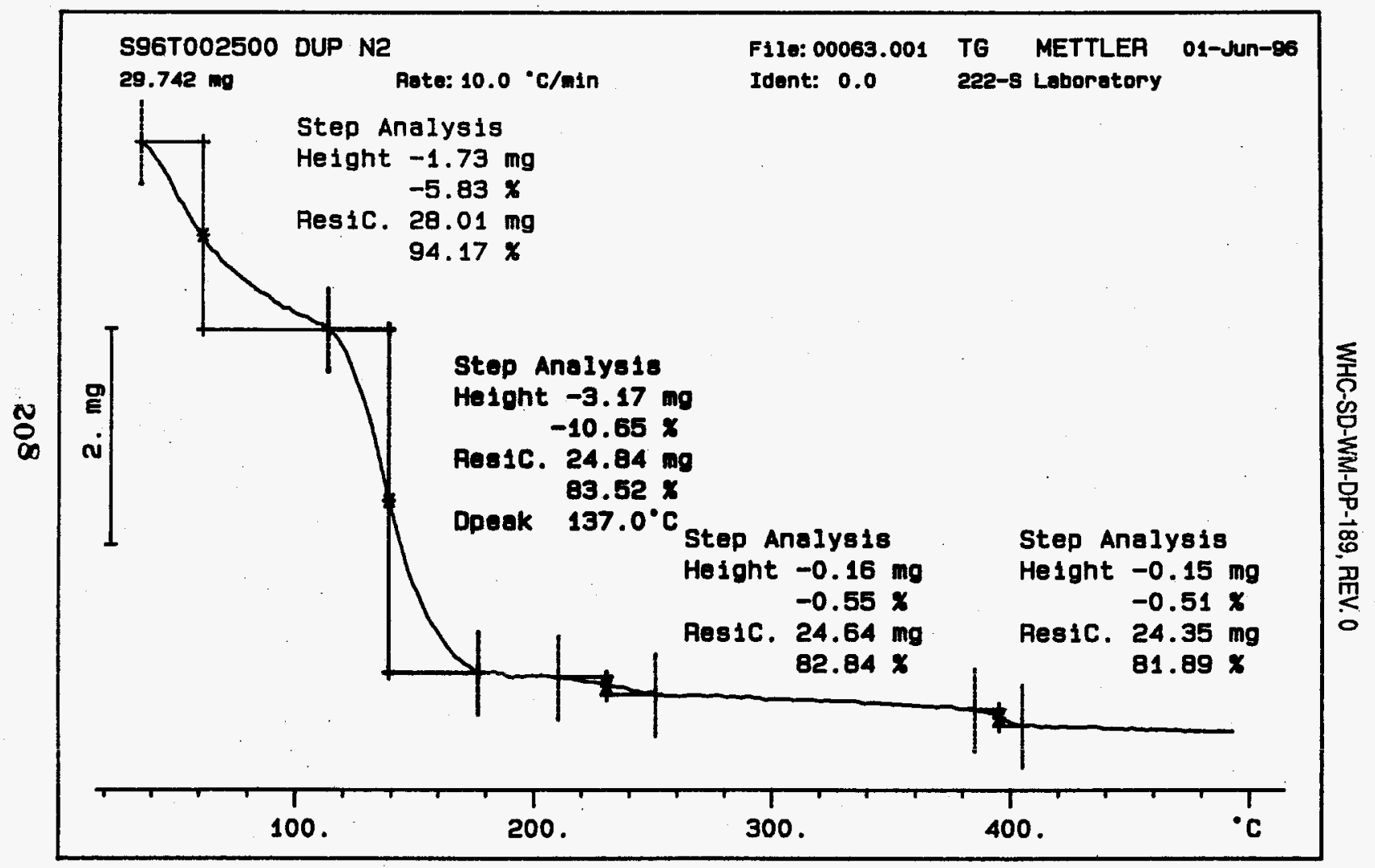




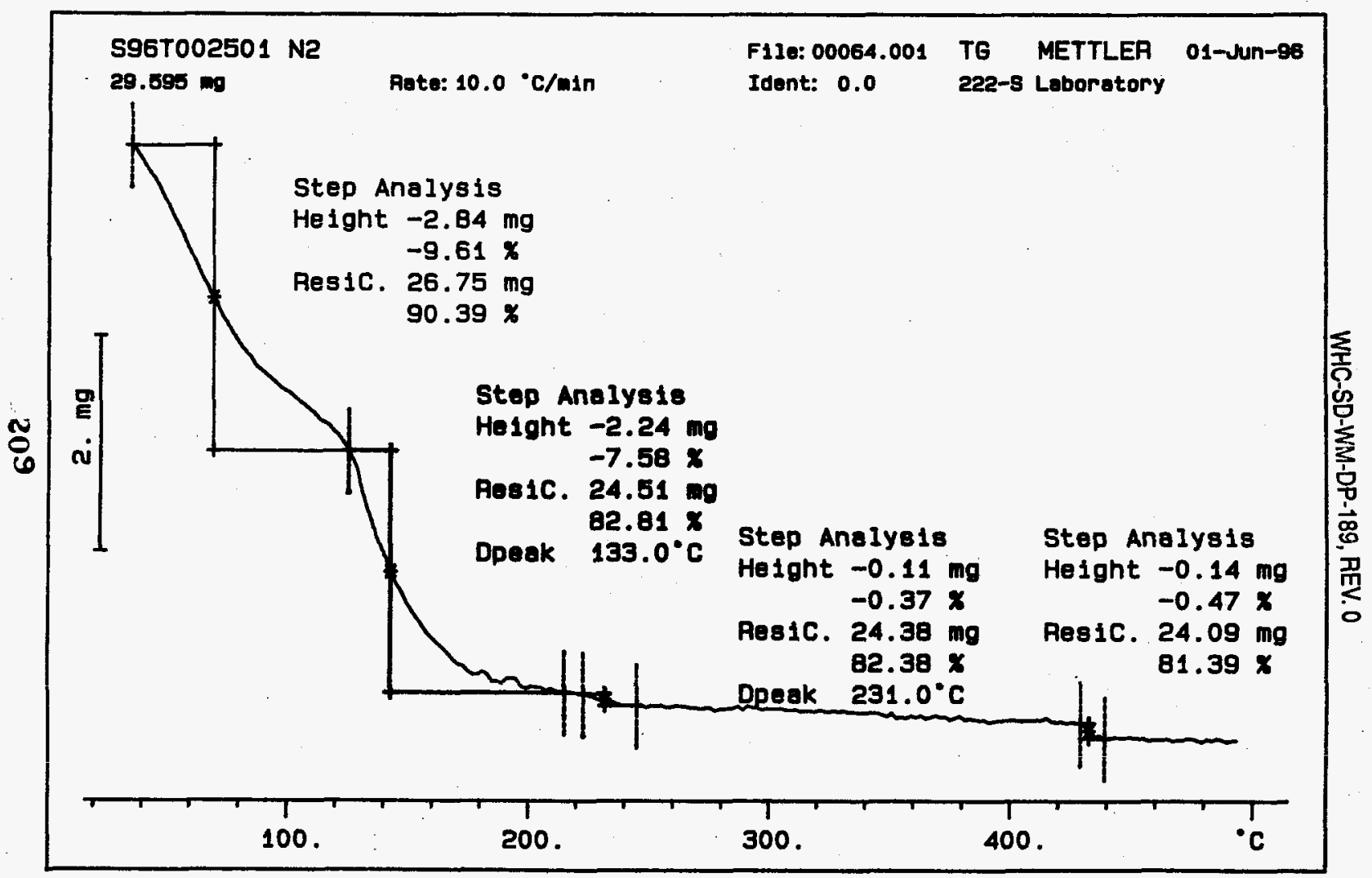




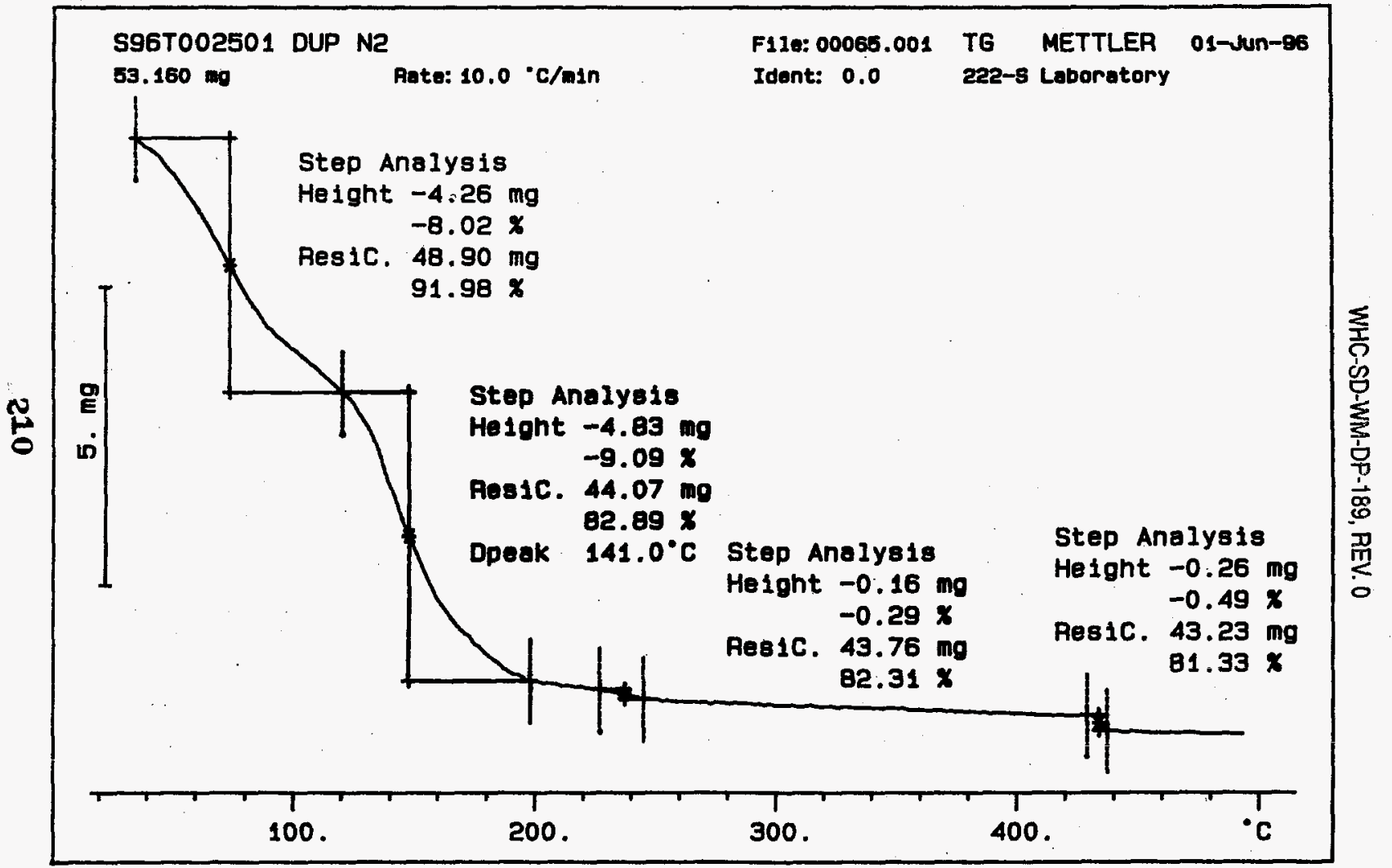




Analyst: SMF Instrument: TGAO I Book \# 82108A

Method: LA-560-112 Rev/Mod B.I

Worklist Comment: U-102 TGA RUN UNDER N2. RCJ

\begin{tabular}{|c|c|c|c|c|c|c|c|c|c|c|}
\hline EROUP & PROJECT & $\begin{array}{l}5 \text { TYPE } \\
1 \text { STD }\end{array}$ & SAMPLE\# & & TEA-01 & $\begin{array}{l}\text { MATRIX } \\
\text { SOLID }\end{array}$ & $\begin{array}{l}\text { ACTUAL } \\
59.2 \\
\end{array}$ & $58.54^{\text {Fa }}$ & W/A & $\begin{array}{l}\text { UNIT } \\
-x\end{array}$ \\
\hline 96000536 & U- 102 & 2 SAHPLE & $596 \mathrm{~T} 002326$ & 0 & & & w/a & 41.5 & & $x$ \\
\hline 96000536 & $U-102$ & 3 DUP & $596 r 002326$ & 0 & TGA-01 & SOLID & 41.5 & 40.3 & M/A & $-x$ \\
\hline
\end{tabular}

Final page for worklist \# 9389

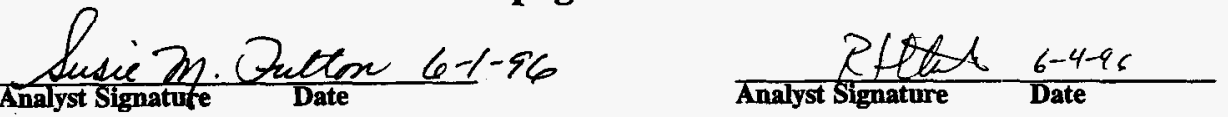
Validated by Hanaotos 6/ulos

Data Entry Comments:

Units shown for $Q C$ (SPK \& STD) may not reflect the actual units. $D L=$ Detection Limit, $S=$ Worklist Slot Number, $R=$ Replicate Number, $A=$ Aliquot Code. 


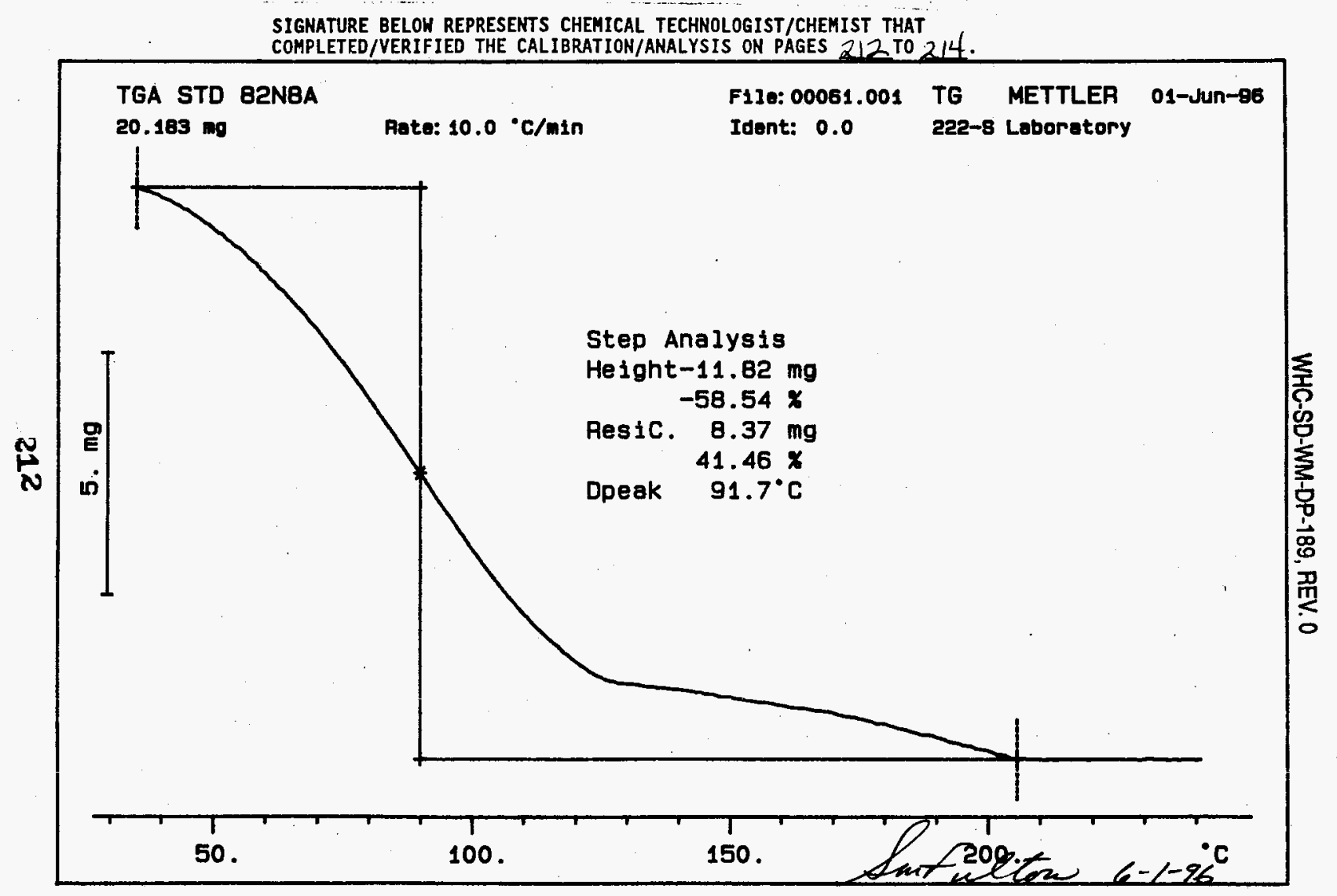




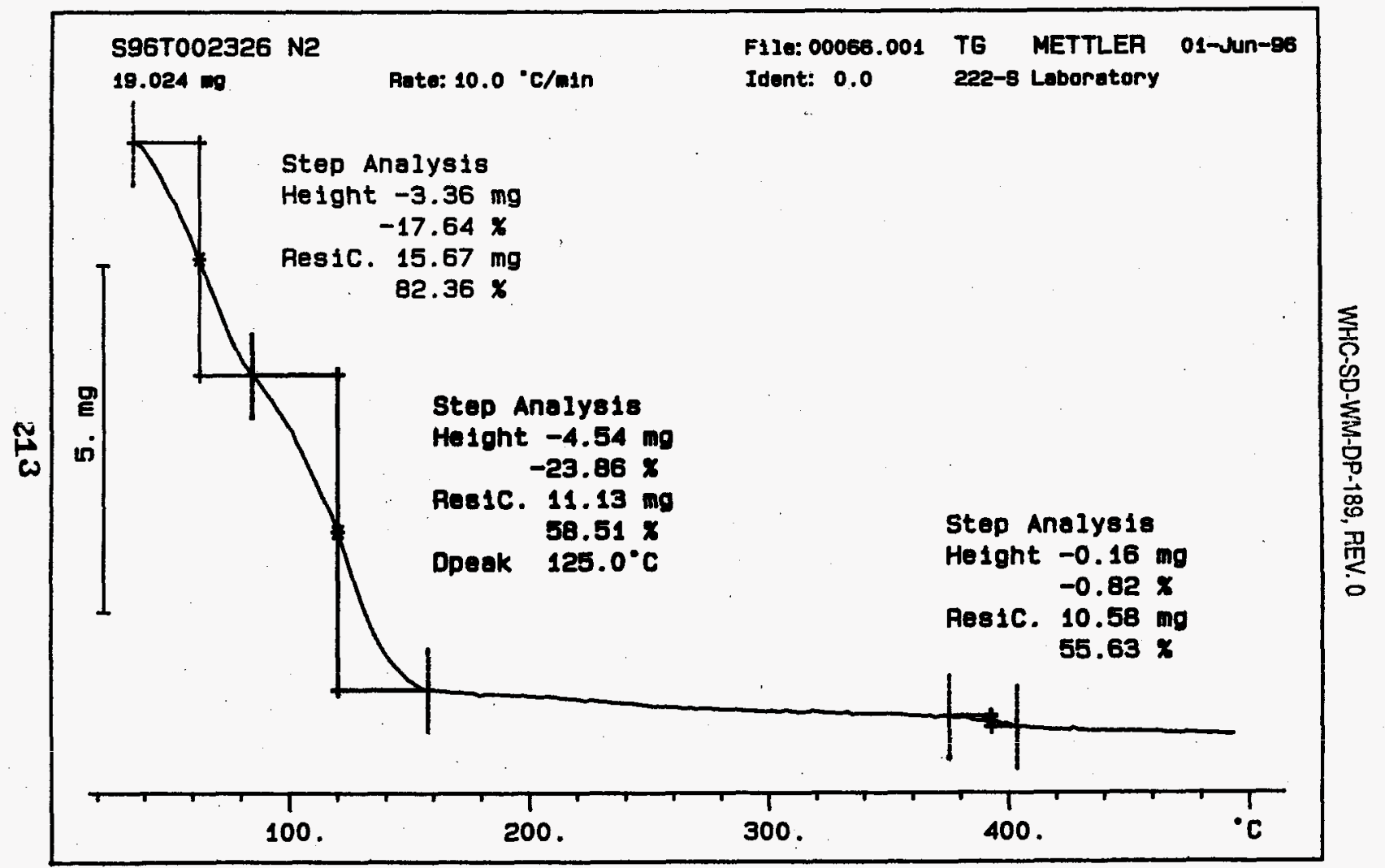




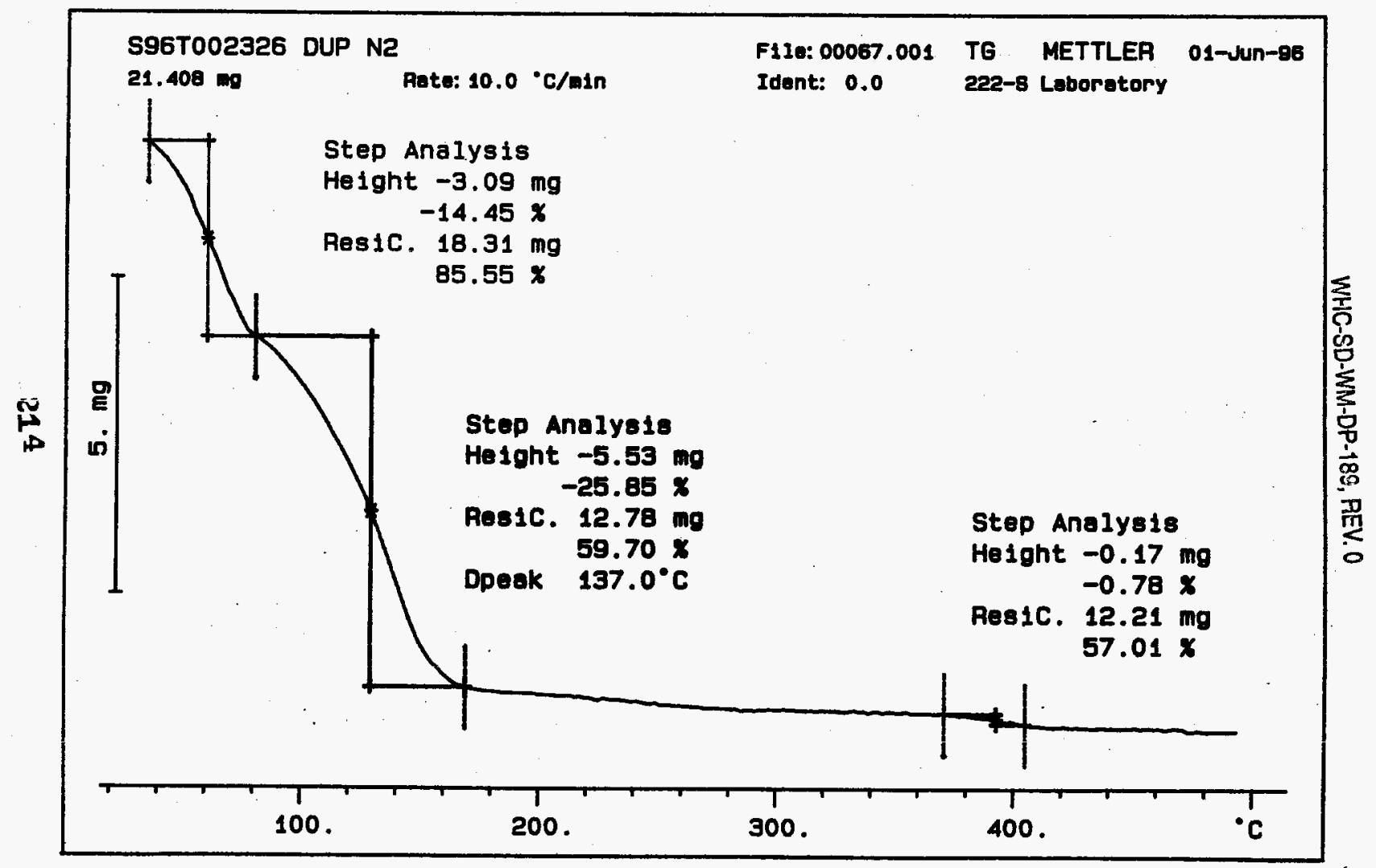




\section{LABCORE Data Entry Template for Worklist\# \\ LABCORE Data Entry Template for Worklist}

Analyst: DCD Instrument: TGA0 I Book \# 82N8A

Method: LA-560-112 Rev/Mod B-1

Worklist Comment: U-102 TGA RUN UNDER N2. RCJ

\begin{tabular}{|c|c|c|c|c|c|c|c|c|c|c|}
\hline croup & PROJECT & $\begin{array}{l}\text { S TYPE } \\
1 \text { STD }\end{array}$ & SAMPLEE & & TEA-01 & $\begin{array}{l}\text { MATRIX } \\
\text { LIQuID }\end{array}$ & $\begin{array}{l}\text { ActuAL } \\
59.2 \\
\end{array}$ & $\begin{array}{l}58.24 \\
\end{array}$ & $N / A$ & $\begin{array}{l}\text { UnIT } \\
x\end{array}$ \\
\hline 96000569 & $U-102$ & 2 SNAPLE & 5961002549 & 0 & teA-01 & LIQUID & $M / 4$ & 52.29 & & $x$ \\
\hline 96000569 & $u-102$ & 3 DUP & 5961002549 & 0 & TGA-01 & LIQUID & 52.29 & 40.23 & N/A & $-x$. \\
\hline 96000569 & U-102 & 4 SAHPLE & 5967002762 & 0 & TGA-01 & LIOUID & N/A & 99.03 & & $x$ \\
\hline 96000569 & U-102 & 5 DUP & S96T002762 & 0 & TGA-01 & LIOUID & 99.03 & 99.87 & H/A & $x$ \\
\hline
\end{tabular}

\section{Final page for worklist \# $\quad 9530$}

\section{$\frac{D_{\text {aind }} D_{\text {undam }} 6-5-96}{\text { Date }}$}

Validated by 1 lanastro 6.6.96
Dithot $6-5-96$

Analyst Signature Date

$$
\text { \#, } 15024
$$

Data Entry Comments:

Units shown for $Q C$ (SPX \& STD) may not reflect the actual units. $D L=$ Detection Limit, $S=$ Worklist Slot Number, $R=$ Replicate Number, $A=$ Aliquot Cade. 
SIGUATURE BEION REPRESENIS CHEMICAL TECGNDLOGIST/CHETIST THAT

COMPLETED/VERIFIED THE CALIBRATION/ANALYSIS ON PAGES 216 T0 220

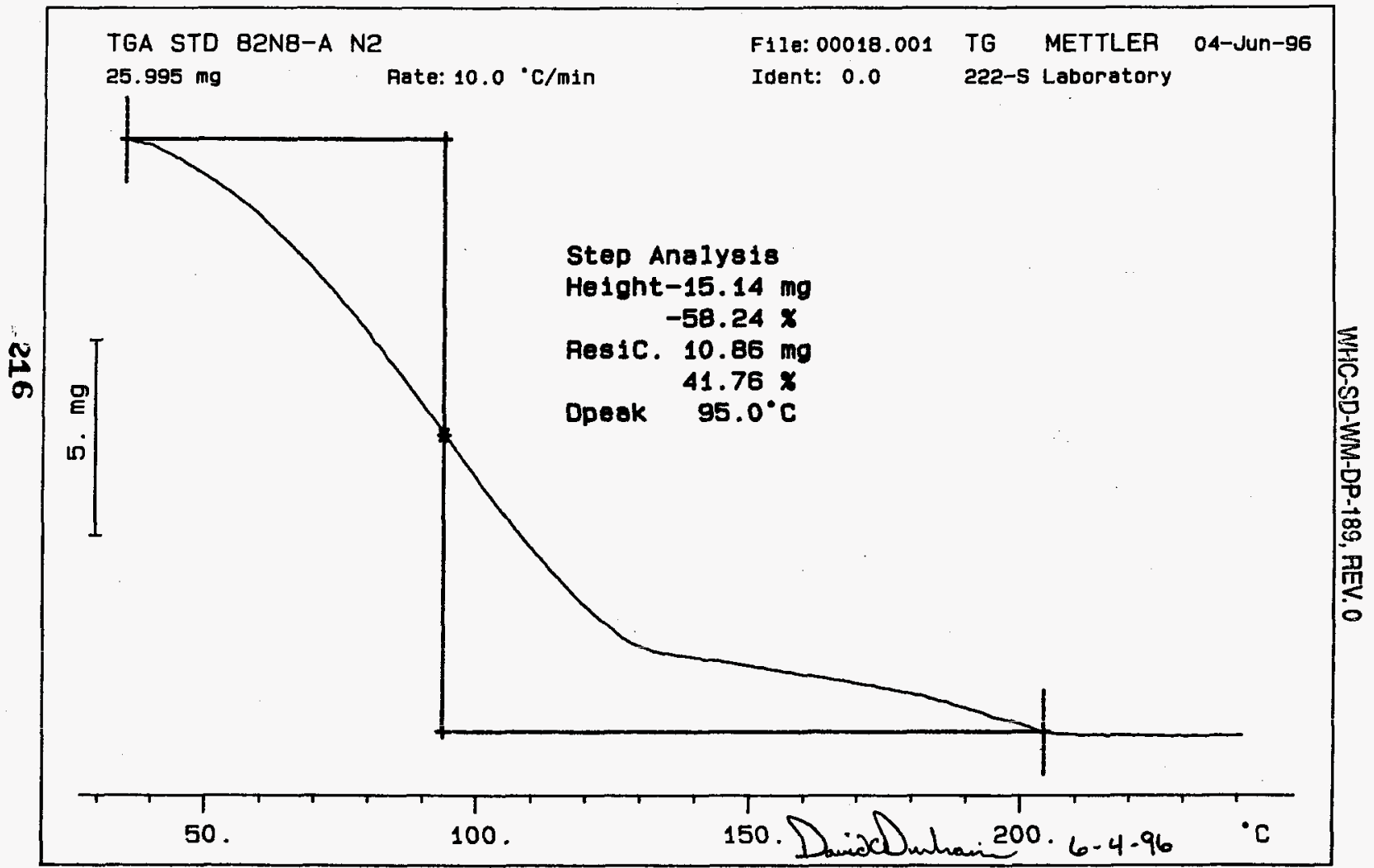




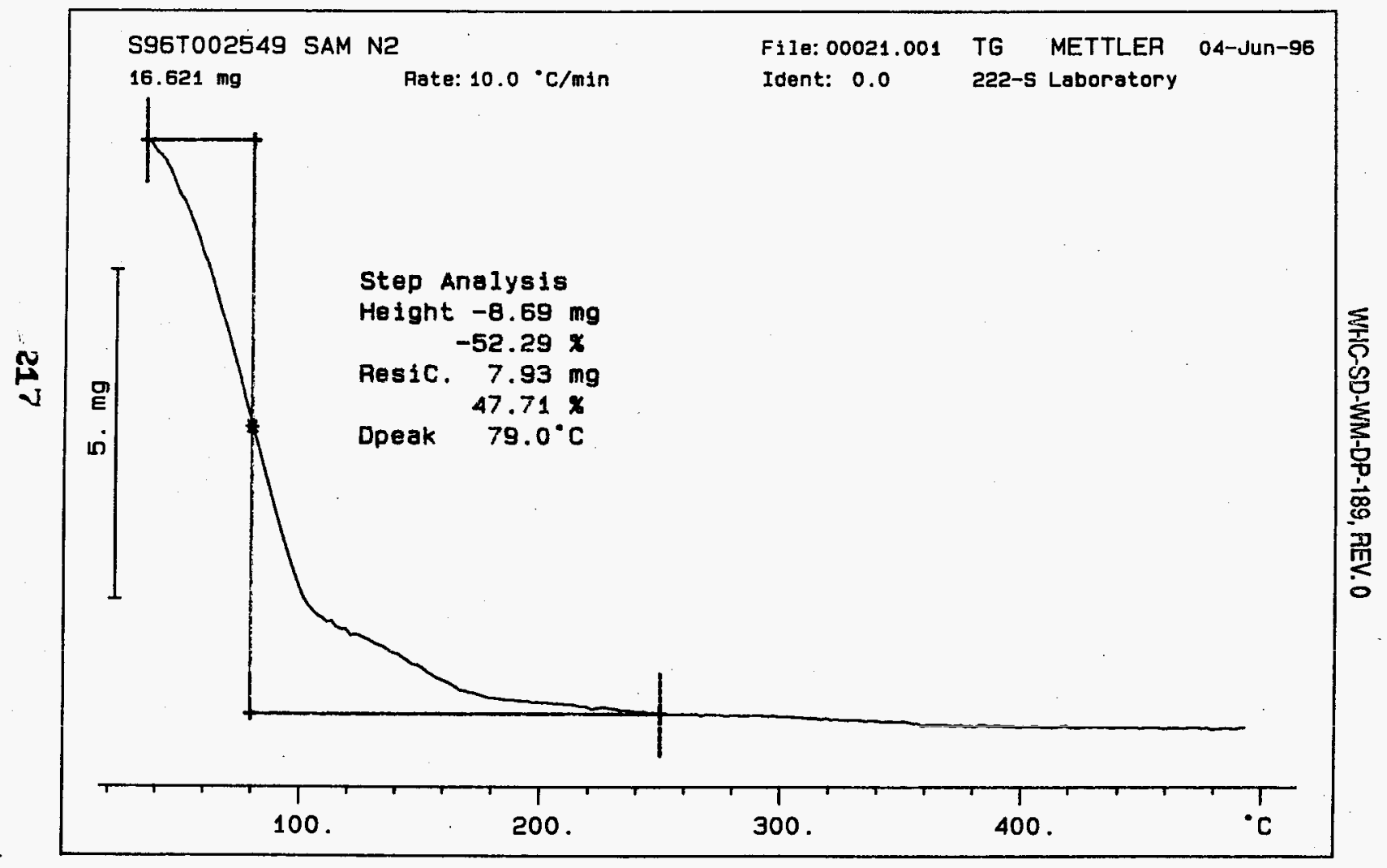




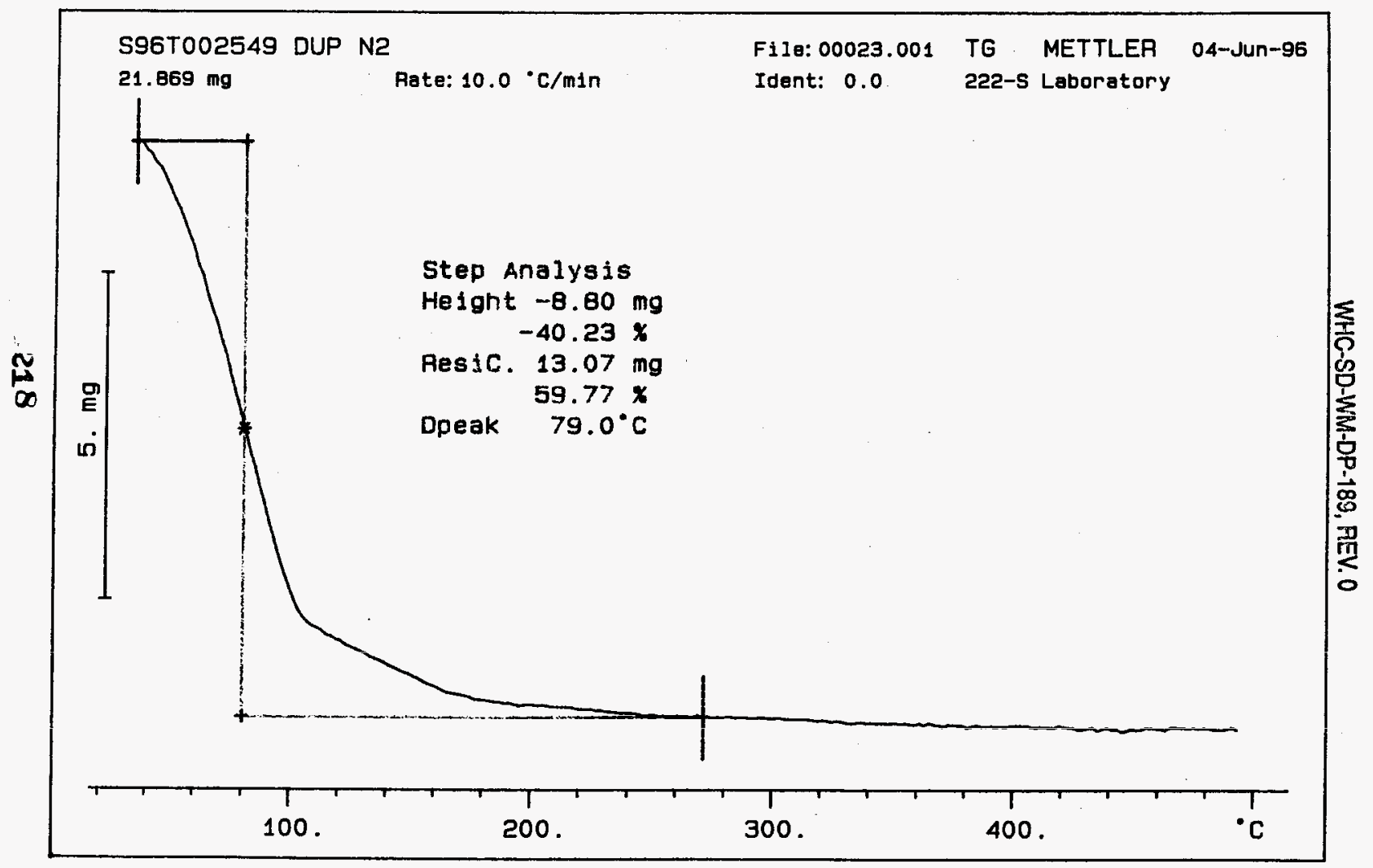




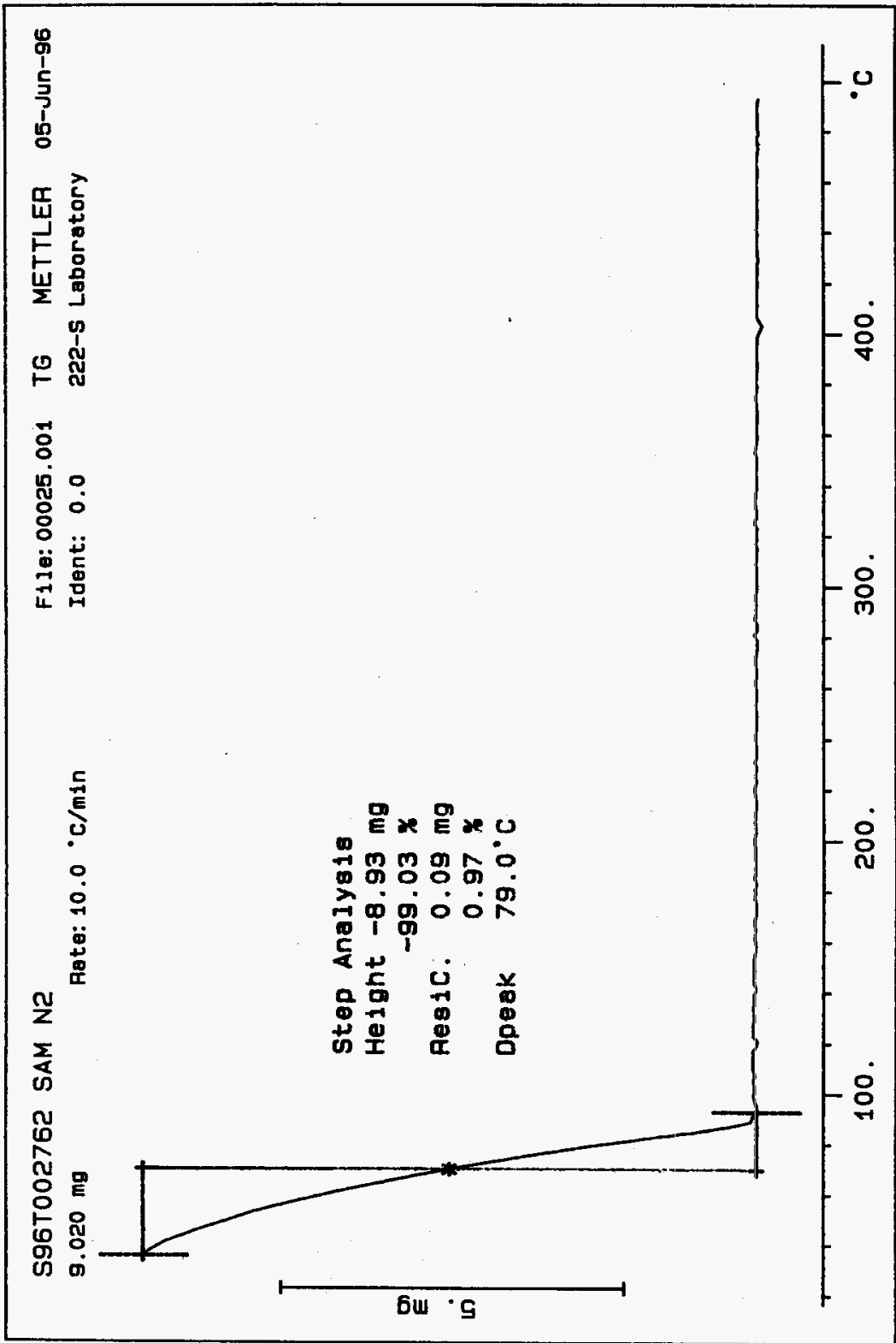


W'TC-SD-WM-DF-189, REV. 0

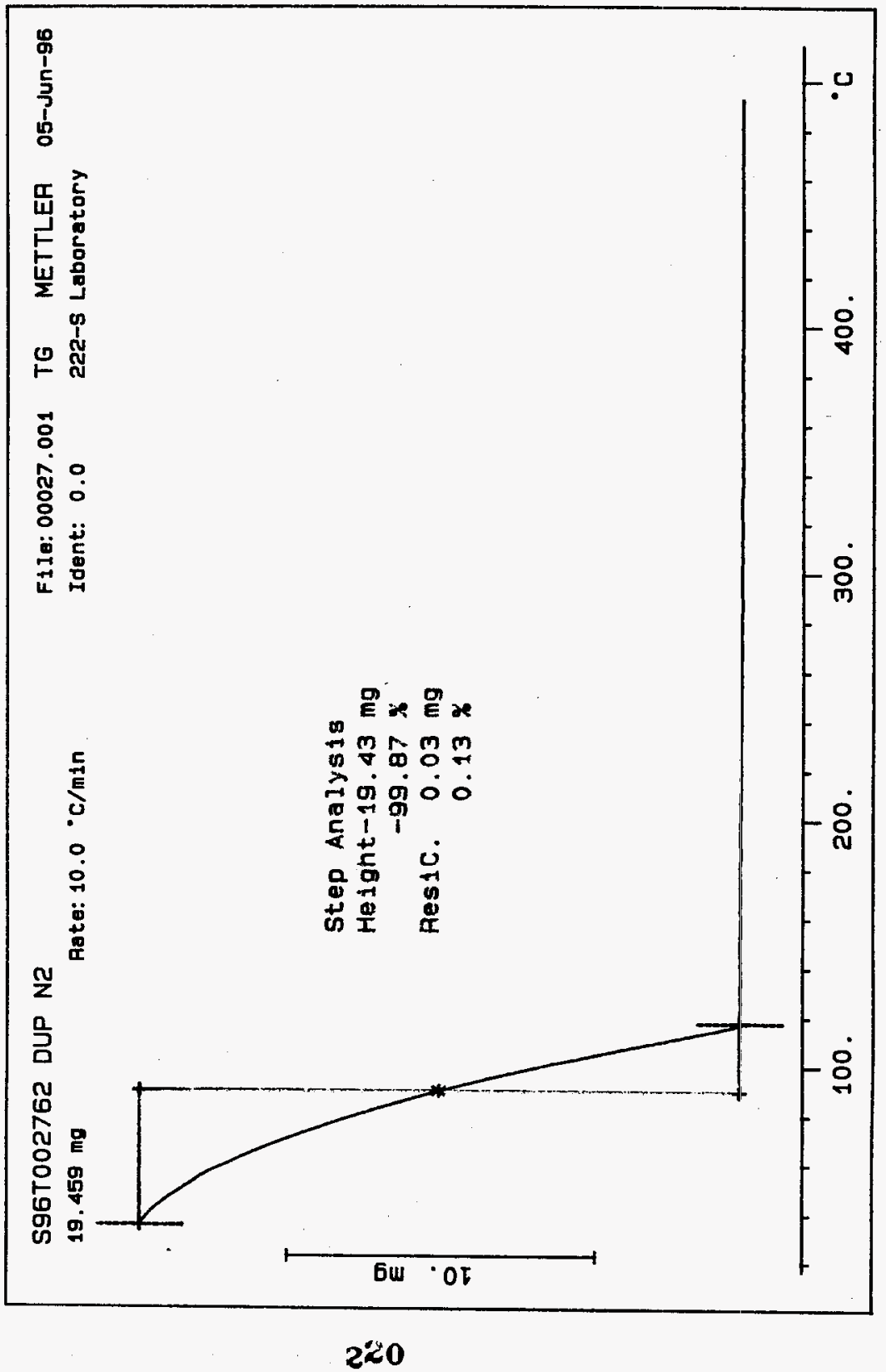


worklistrpt Version 2.1 05/15/95

WHC-SO-WWR-DP-189, REV. 0

Page: I

$06 / 03 / 9614: 31$

LABCORE Data Entry Template for Worklist

9531

Analyst: $\quad D C D$ Instrument: TGA0 I Book \# BZN8B

Method: LA-560-112 Rev/Mod B-I

Worklist Comment: U-102 TGA RUN UNDER N2. RCJ

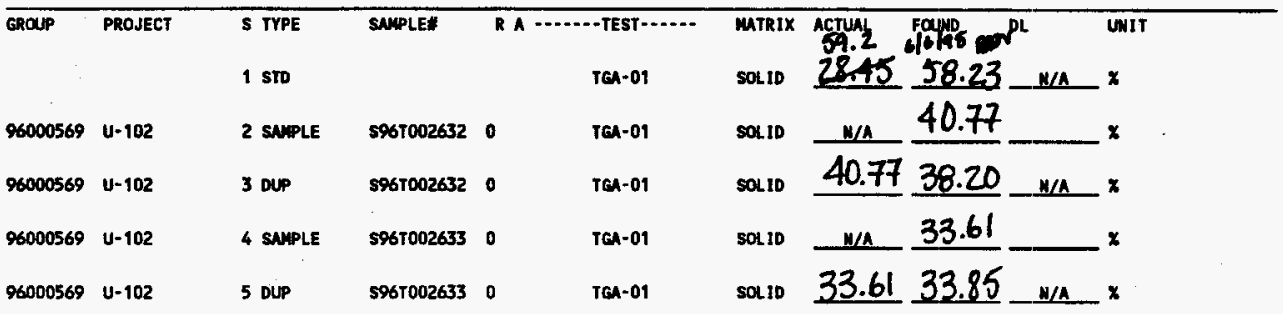

Final page for worklist \# 9531

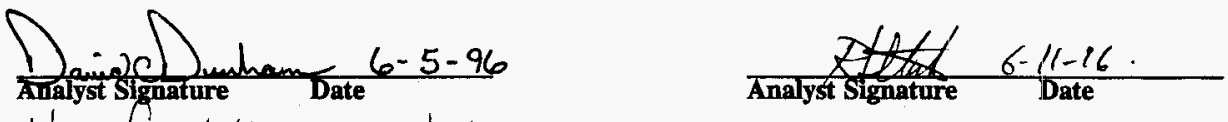

Verifica/Validated by

Blandina

Valenzuela $1-12.96$

Data Entry Comments:

Units shown for QC (SPK \& STD) may not reflect the actual wits. DL = Detection Limit, $S=$ Worklist Slot huber, $R=$ Replicate Number, $A=A$ aliquot Code.

221 


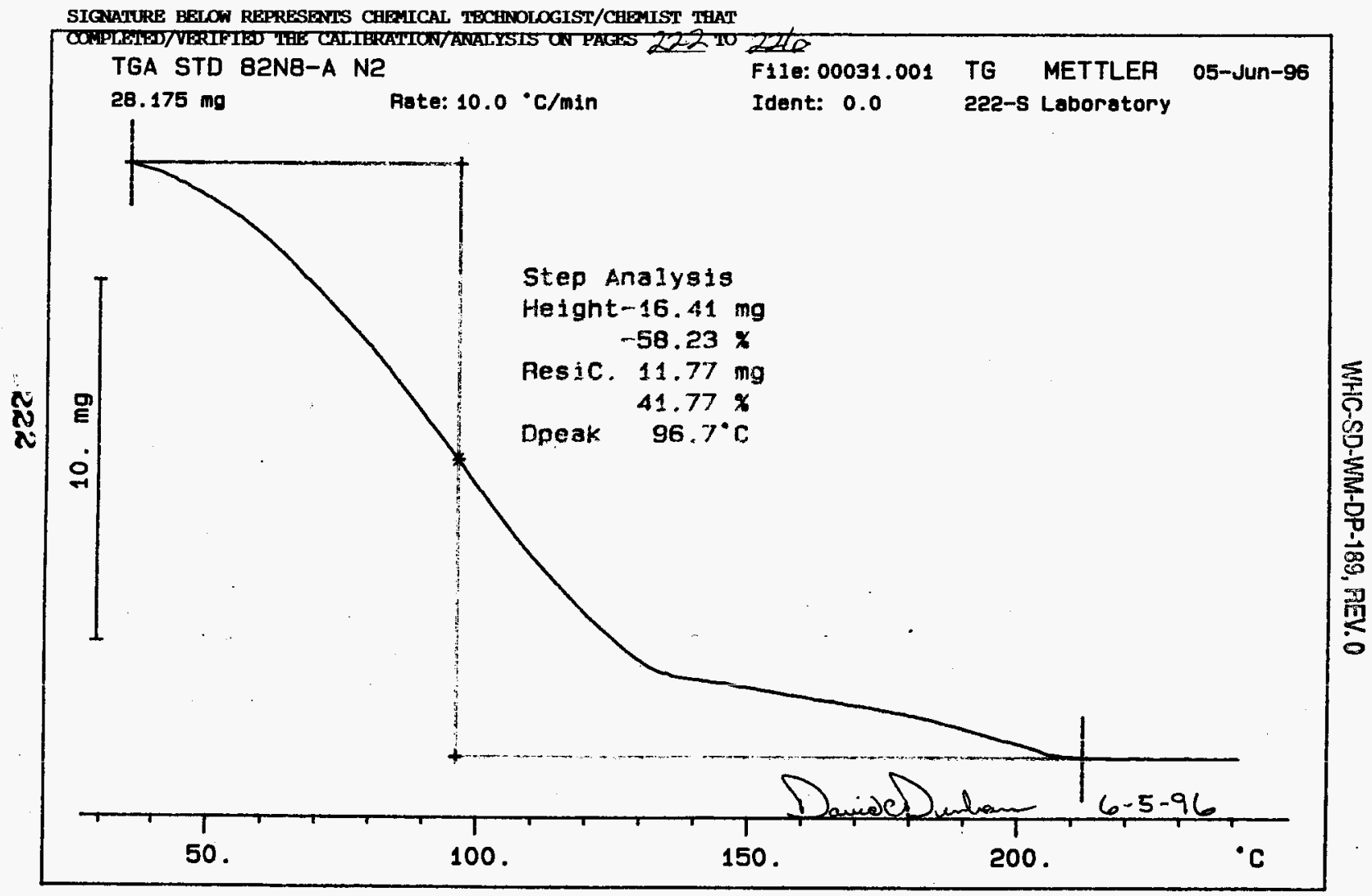



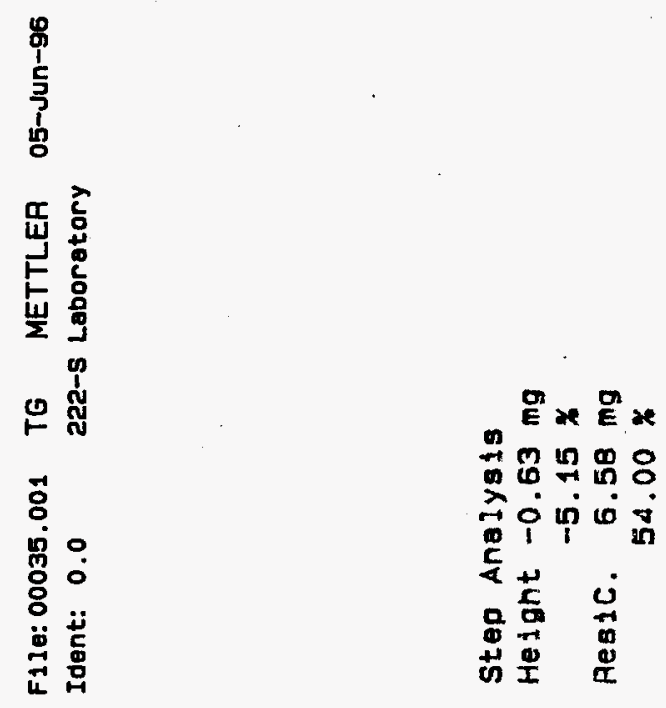

돌

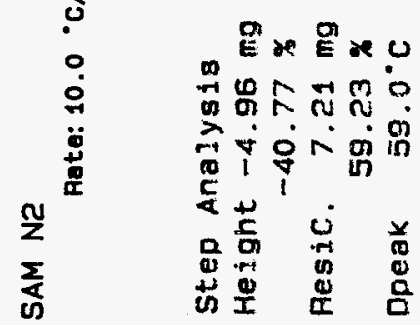

N

ก

o

ณิ

웅

to

ถู ญี
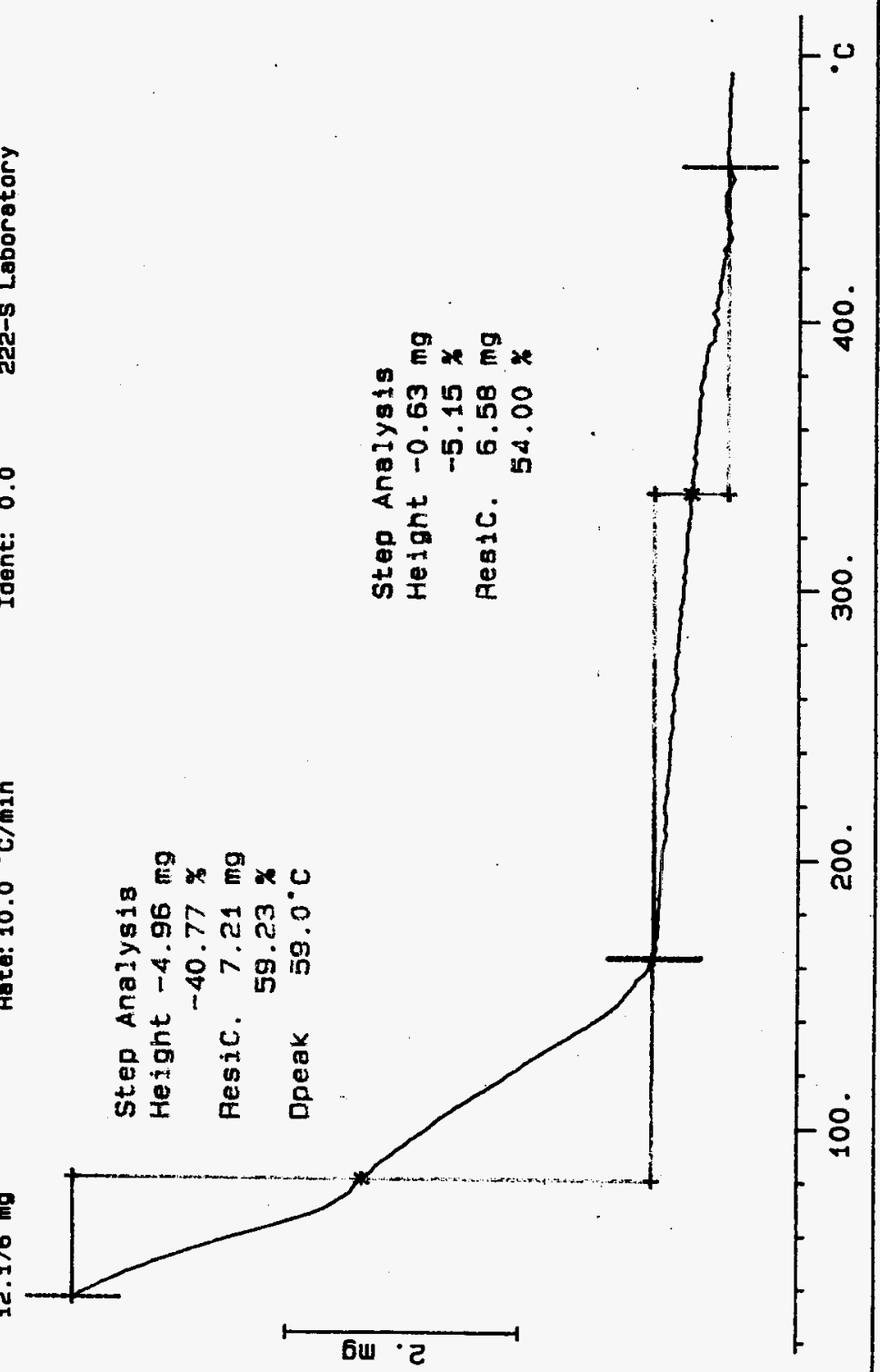


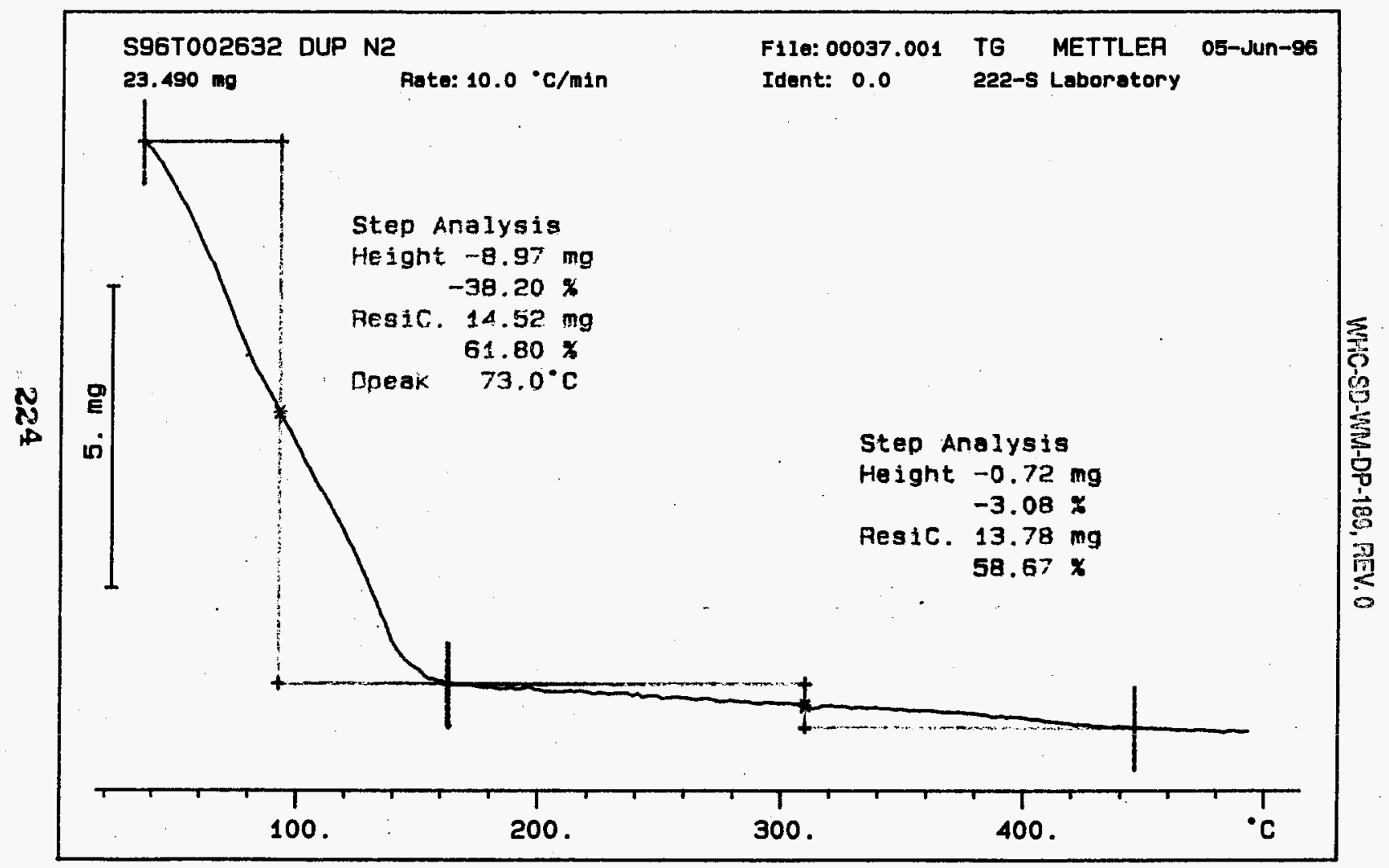




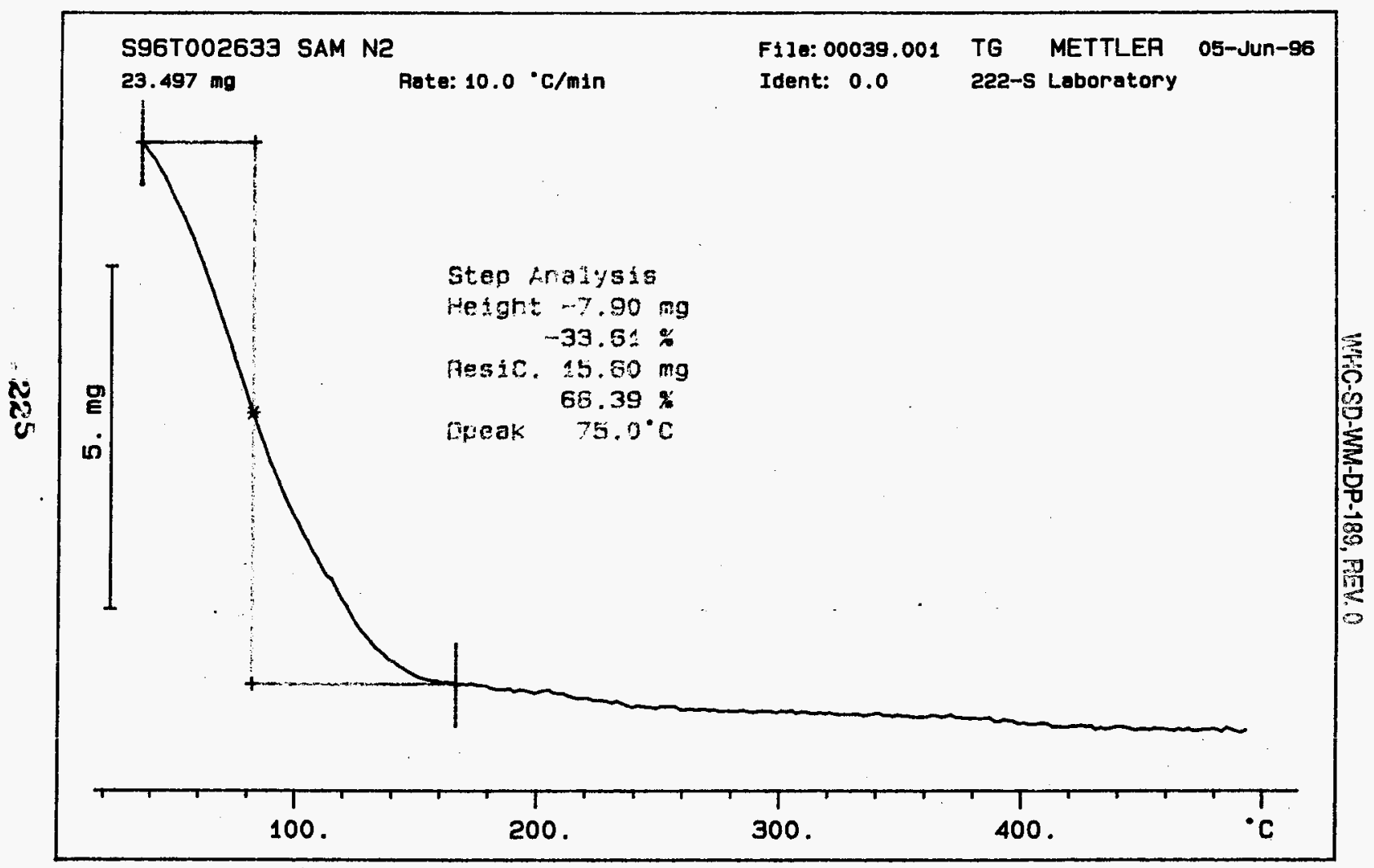




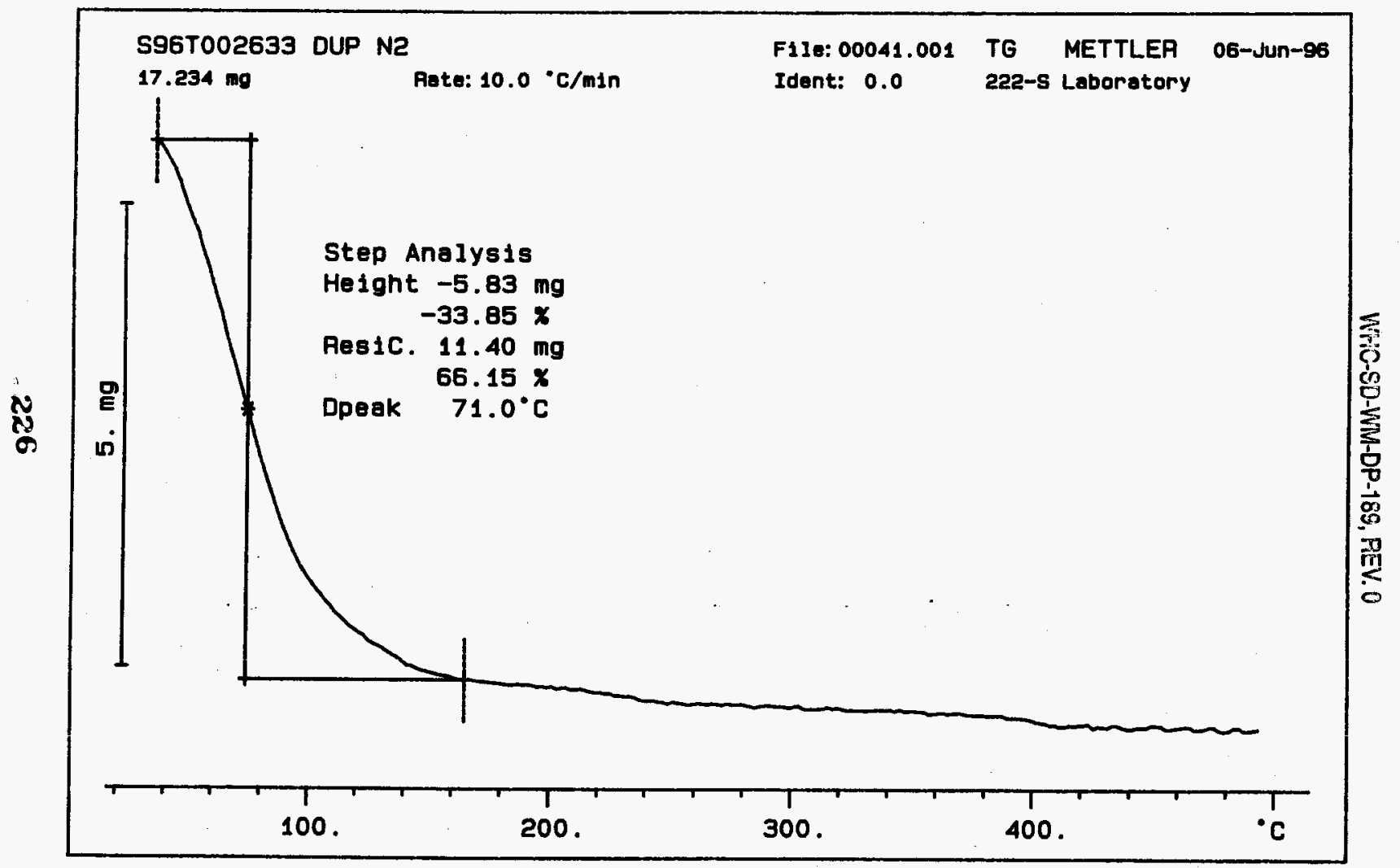


worthistpt Version 2.1 05/15/95 WHC-SD-WM-DP-189, REV. 0
$06 / 11966$ 14:18

Page: 1

LABCORE Data Entry Template for Worklist\#

9532

Analyst: $\quad$ DCD Instrument: TGA0 3 Book \#8 2NBA

Method: LA-514-114 Rev/Mod C-I

Workhist Comment: U-102 TGA RUN UNDER N2. RCJ

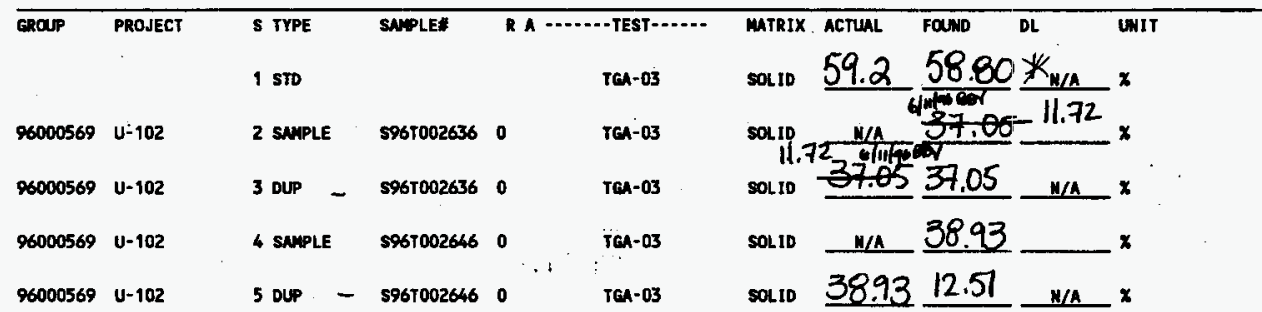

Final page for worklist \# 9532

See attached for signatures

Analyst Signature Date $6-11-96$

Verified/validated by

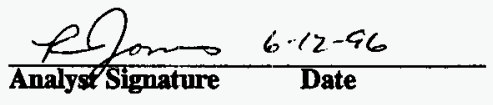

Blandina Valenzuela 6/13/96

S96702636 will be sum in replicate due to the targe difference

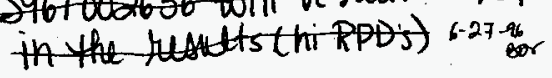

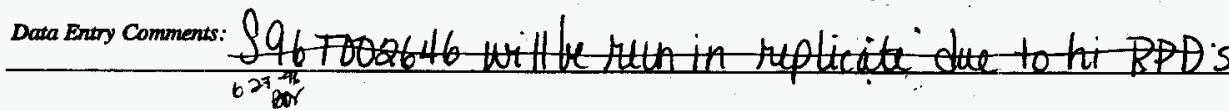

Units shown for $Q C$ (SPK \& STD) may not reflect the actual units. $D L=$ Detection Limit, $S=$ Worklist Shot Number, $R=$ Replicate Number, $A=$ Aliquot Cade.

227 


\section{LABCORE Data Entry Template for Worklist\#}

Analyst: DCP Instrument: TGA0 Book $82 \mathrm{~N} 8 \mathrm{~A}$

Method: LA-560-112 Rev/Mod

Worklist Comment: U-102 TGA RUN UNDER N2. RCJ

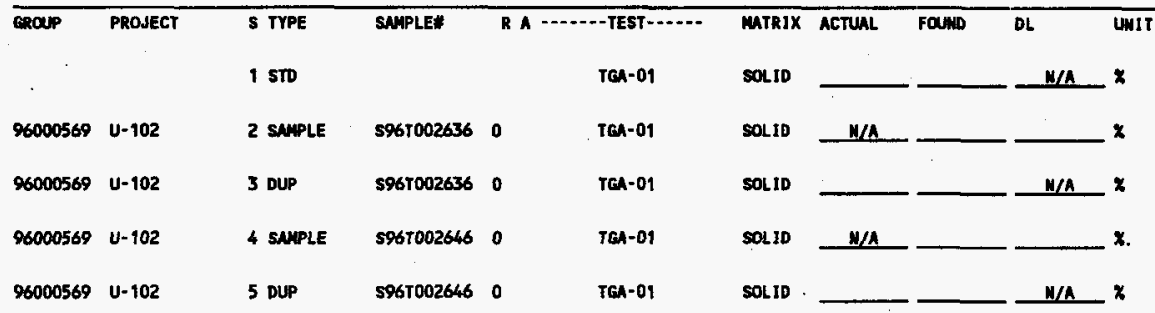

Final page for worklist \# 9532

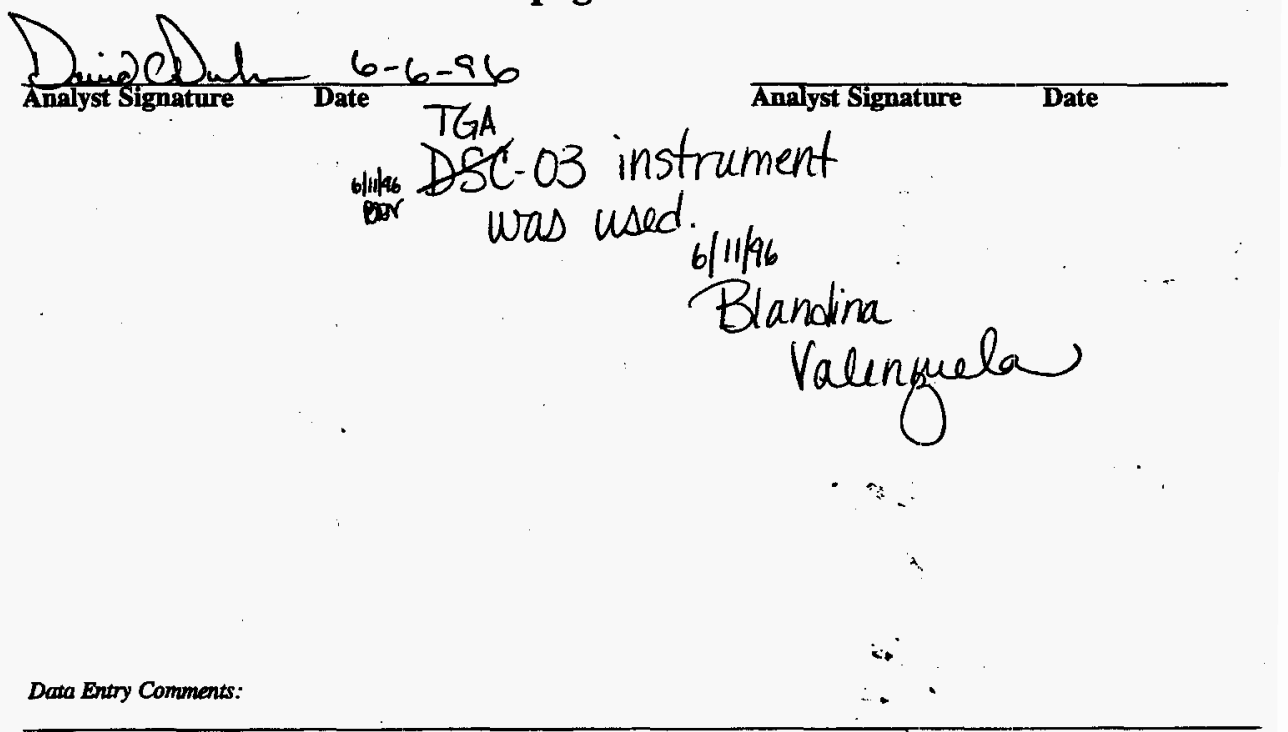

Units shown for $Q C$ (SPK \& STD) may not reflect the actual units. $D L=$ Detection Limit, $S=$ Worklist Slot Number, $R_{.}=$Replicate Number, $A=$ Aliquot Code. 
Curve 1: TEA

F1le info: TER060601 Thu Jun 6 20: 46:00 1996

Samplo Waight: 17.685

a

TGA STD BENB-A

SIGNATURE BELOW REPRESENTS CHEMICAL TECHNOLOGIST/CHEMIST THAT

COMPLETED/VERIFIED THE CALIBRATION/ANALYSIS ON PAGES 2290203

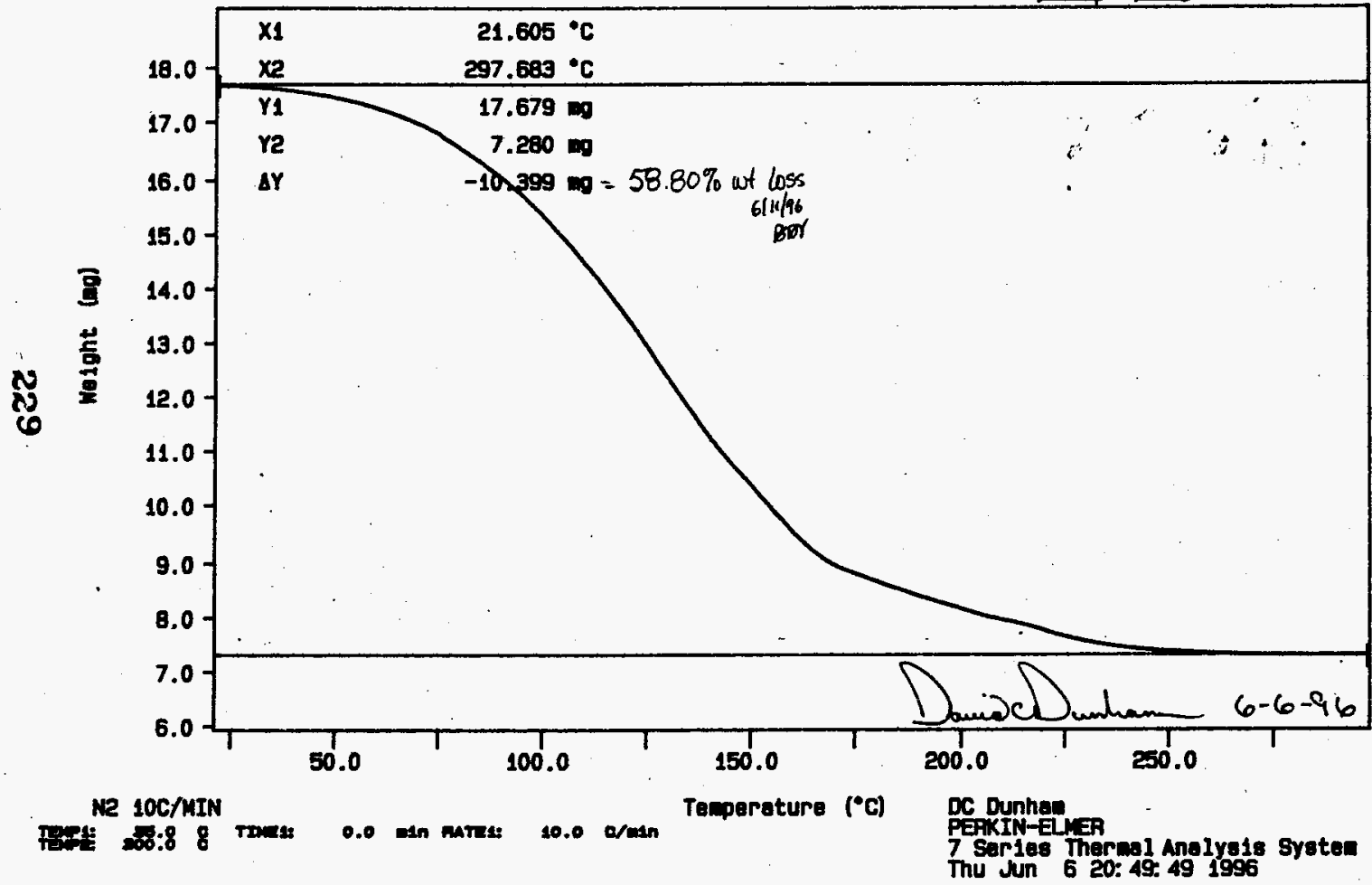


Curve 1: TEA

F110 info: sume0603 Thu un 6 19:07: 241996

Saple Waight: 22.760 ind

sesto026s8 SN4

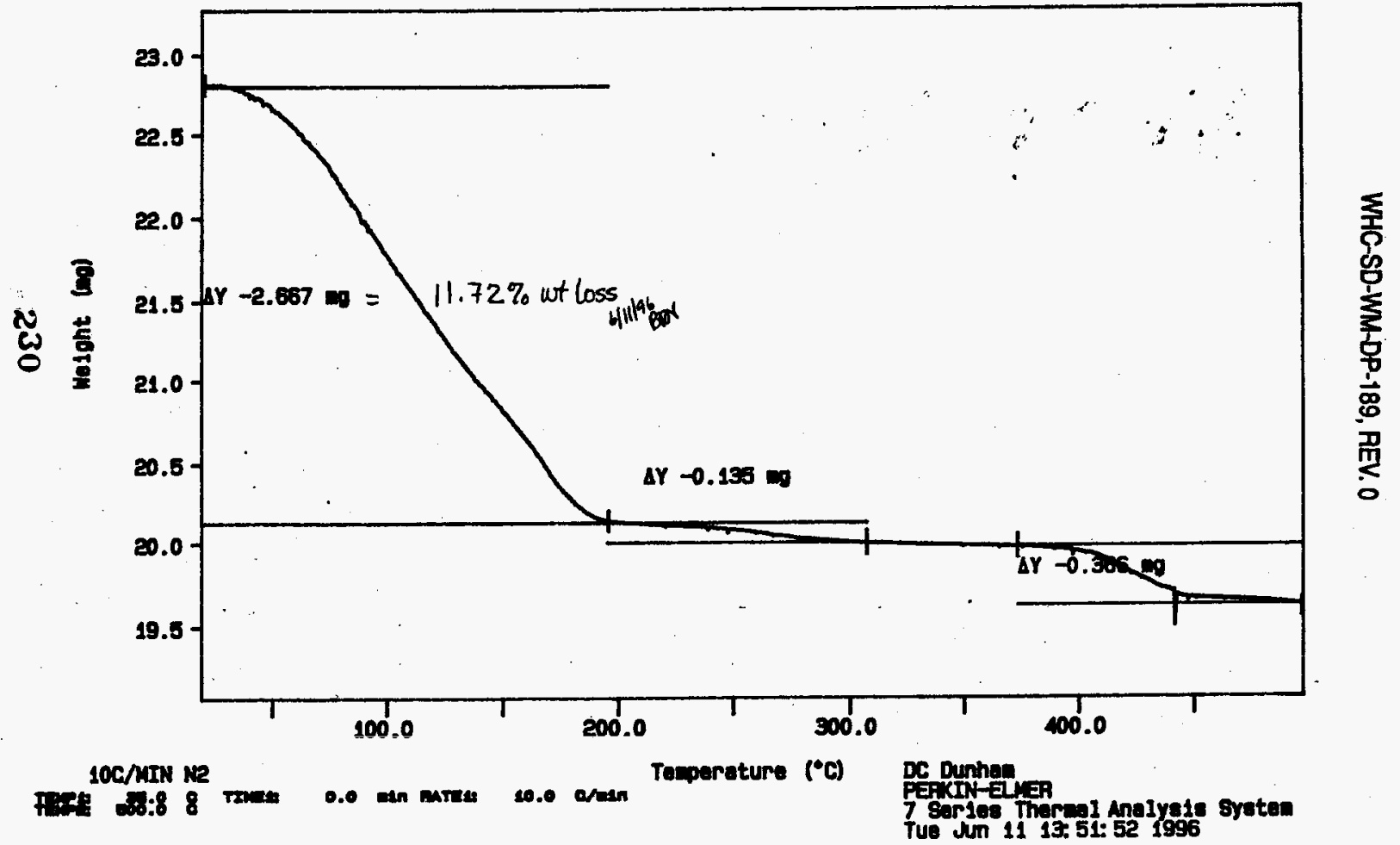


WHC-SD-WM-DP-189, REV. 0

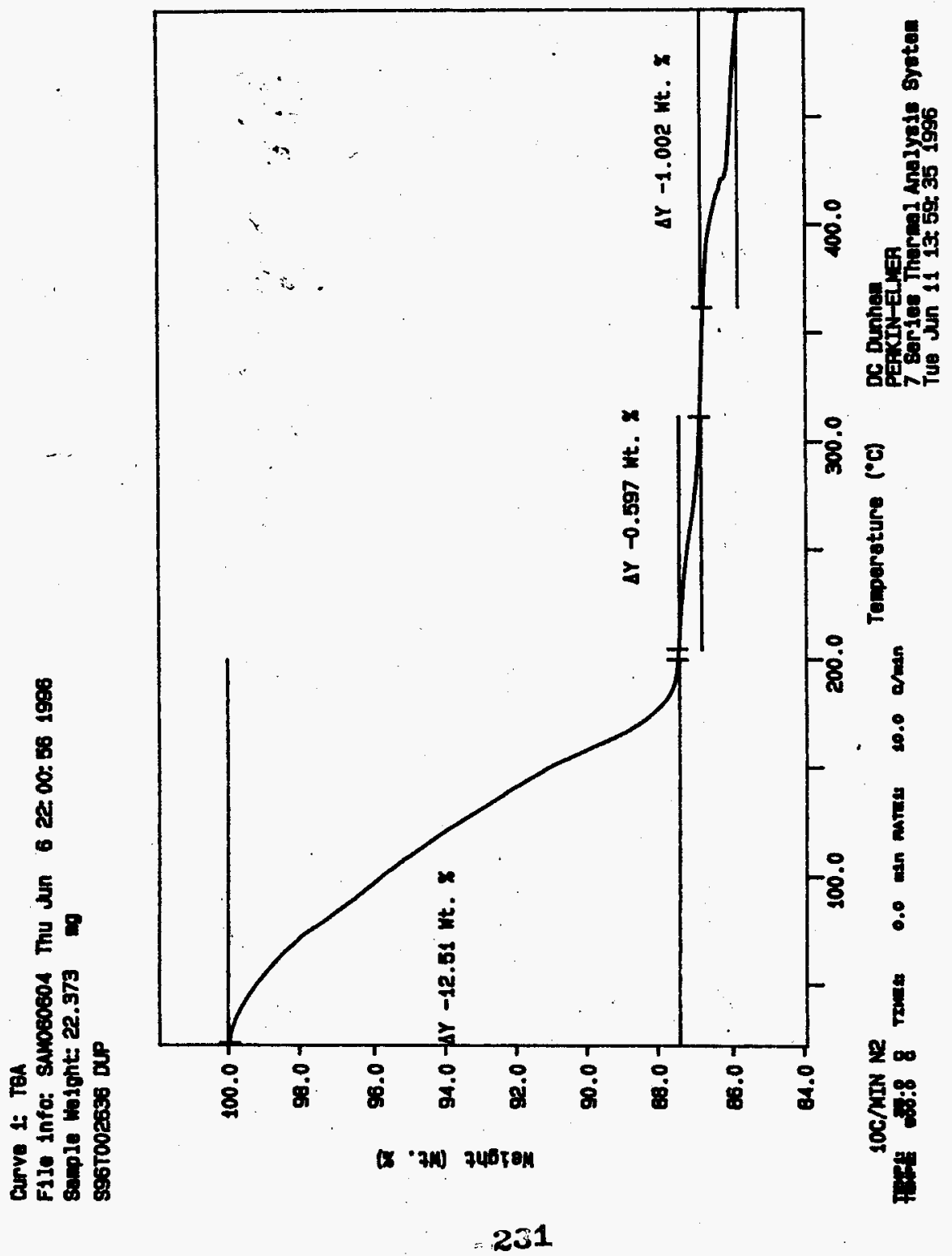


WHC-SD-WM-DP-189, REV. 0

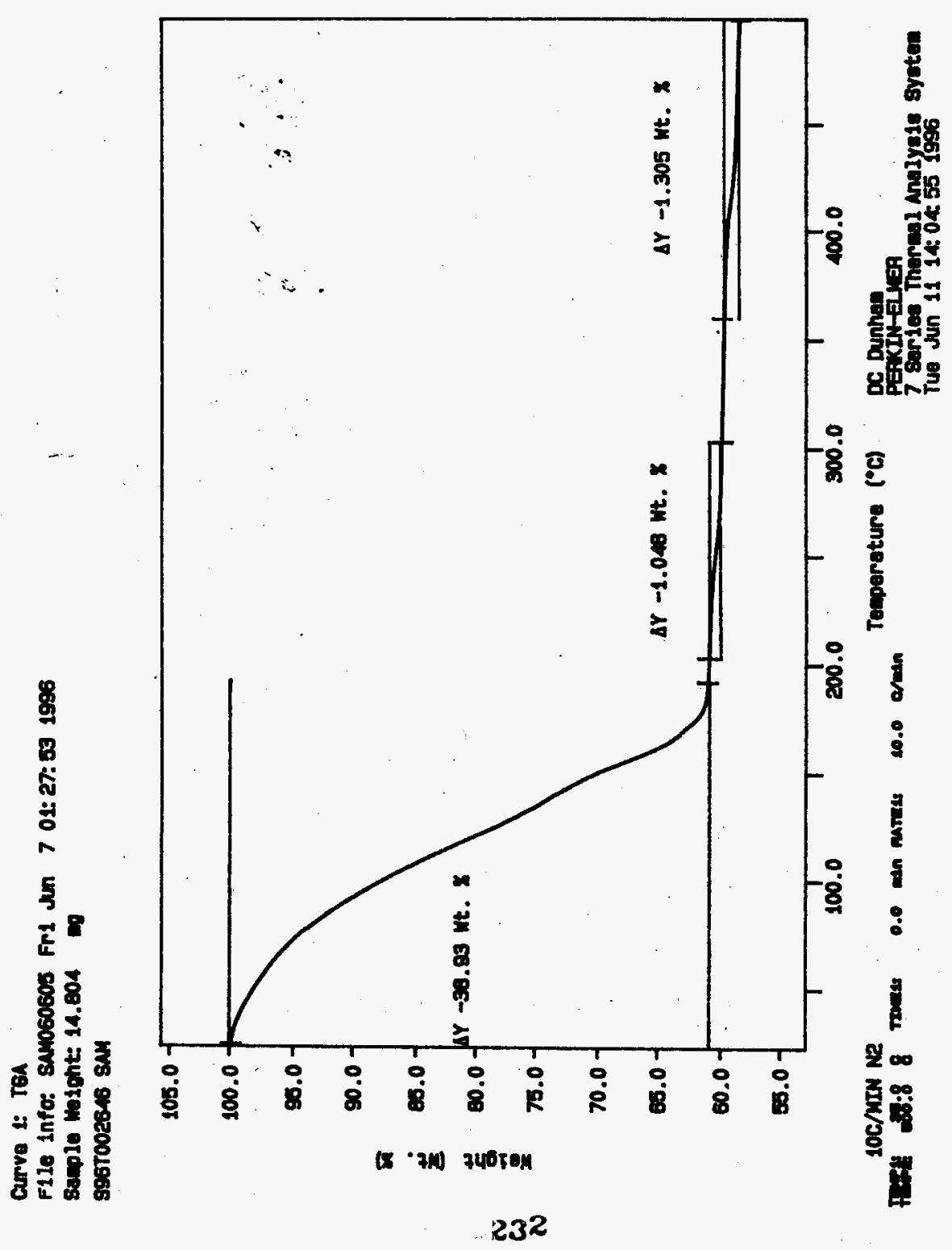


WHC-SD-WM-DP-189, REV. 0

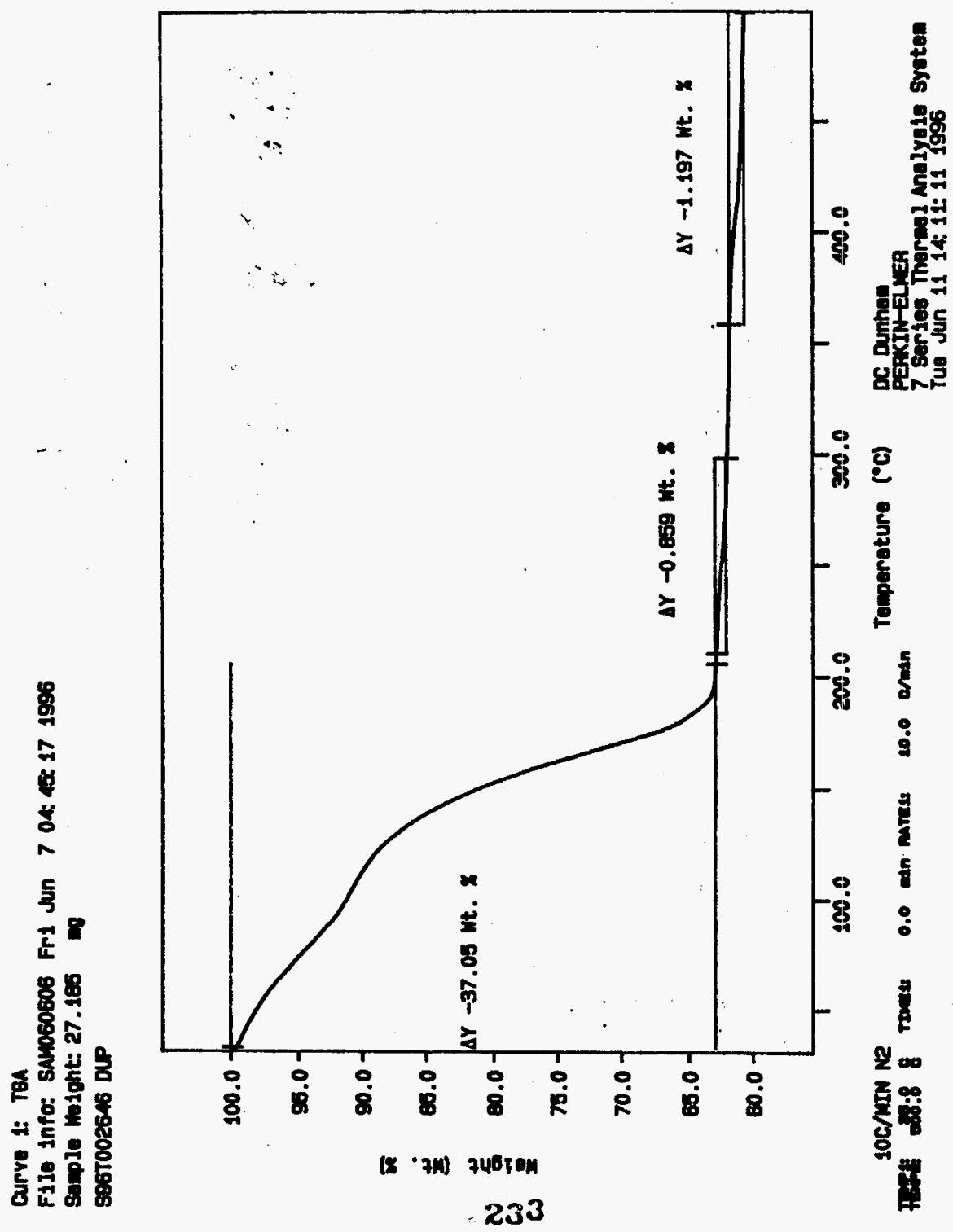




\section{LABCORE Data Entry Template for Worklist\#}

Analyst: DCD Instrument: TGA0 I Book \# 82N8A

Method: LA-560-112 Rev/Mod B-1

Worklist Comment: U-102 TGA RUN UNDER N2. RCJ

\begin{tabular}{|c|c|c|c|c|c|c|c|c|c|c|}
\hline \multirow[t]{2}{*}{ GROUP } & \multirow[t]{2}{*}{ PROJECT } & \multirow{2}{*}{$\begin{array}{l}\text { S TYPE } \\
\text { I STO }\end{array}$} & \multirow[t]{2}{*}{ SAMPLE" } & \multicolumn{2}{|c|}{ R A $\cdots$ - } & \multirow{2}{*}{$\begin{array}{l}\text { MATR IX } \\
\text { SOLID }\end{array}$} & \multirow{2}{*}{$\begin{array}{l}\text { Actual } \\
59.2 \\
\end{array}$} & \multirow{2}{*}{$\begin{array}{l}\text { FOAND } \\
58.23 \\
\end{array}$} & \multirow{2}{*}{$\begin{array}{l}\text { DL } \\
\mathscr{W}_{\text {W/A }}\end{array}$} & \multirow{2}{*}{$x$} \\
\hline & & & & & Ten-01 & & & & & \\
\hline 96000569 & $U-102$ & 2 SNMPLE & 5967002647 & 0 & TEA-01 & SOLID & $\mathbf{m} / \boldsymbol{A}$ & 7.68 & & $x$ \\
\hline 96000569 & $U-102$ & 3 DUP & 596T002647 & 0 & TEA-01 & SOL.10 & 7.68 & 10.46 & $\omega / a$ & $x^{*}$ \\
\hline 96000569 & U-102 & 4 SAMPLE & 5967002775 & 0 & TEA-01 & SOLID & H/A & 24.47 & & $x$ \\
\hline 96000569 & U-102 & 5 DUP & 59670027/5 & 0 & TEA-01 & sol.10 & $24: 4$ & 20.10 & W/A & $x$ \\
\hline
\end{tabular}

\section{Final page for worklist \# $\quad 9533$}
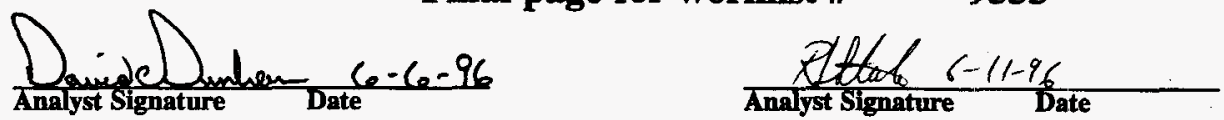

Data Entry Comments:

Units shown for QC (SPK \& STD) may not neflect the actual units. $D L=$ Detection Limit, $S=$ Worklist Slot Number, $R=$ Replicate Number, $A=A$ Aiquot Code. 


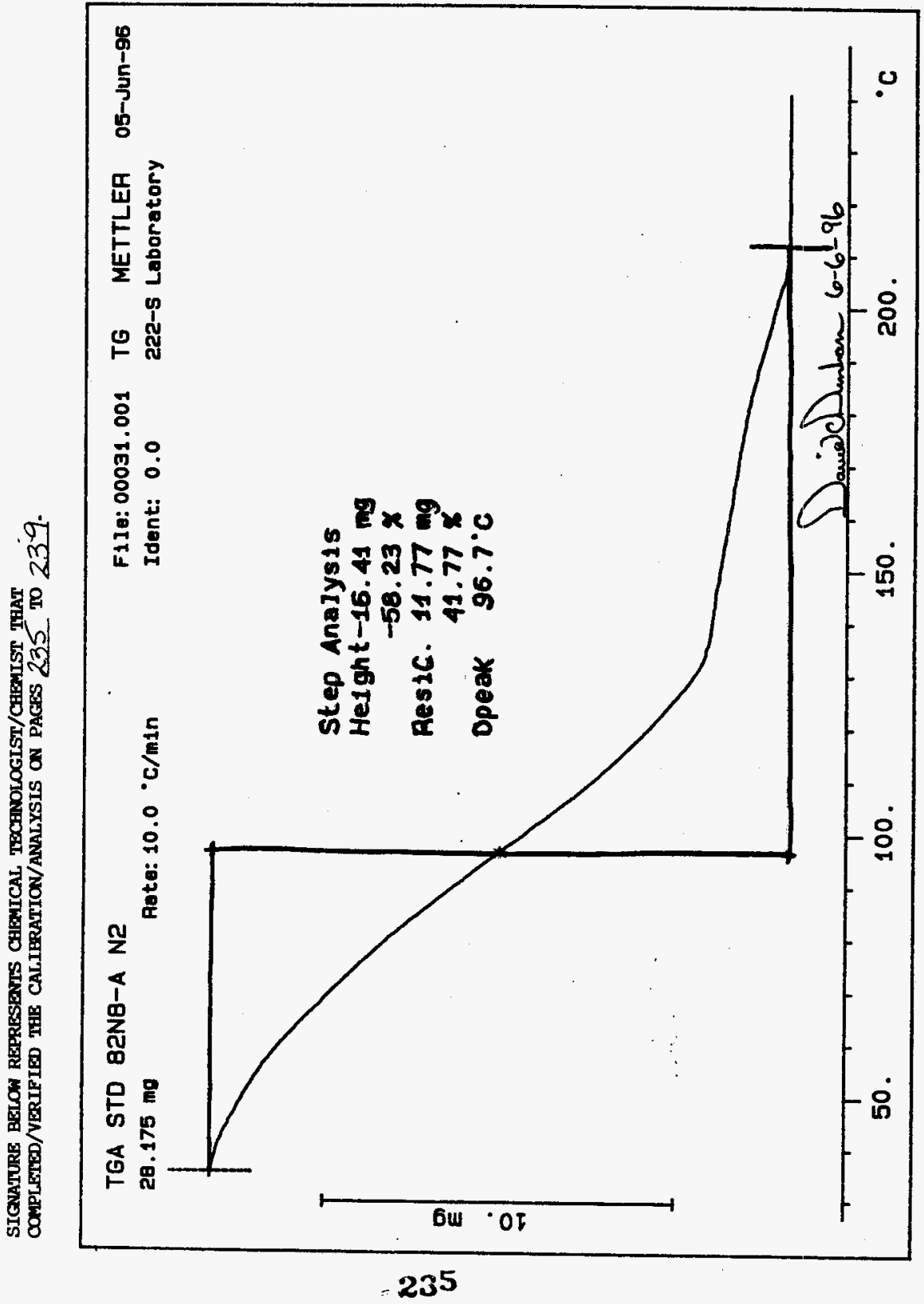




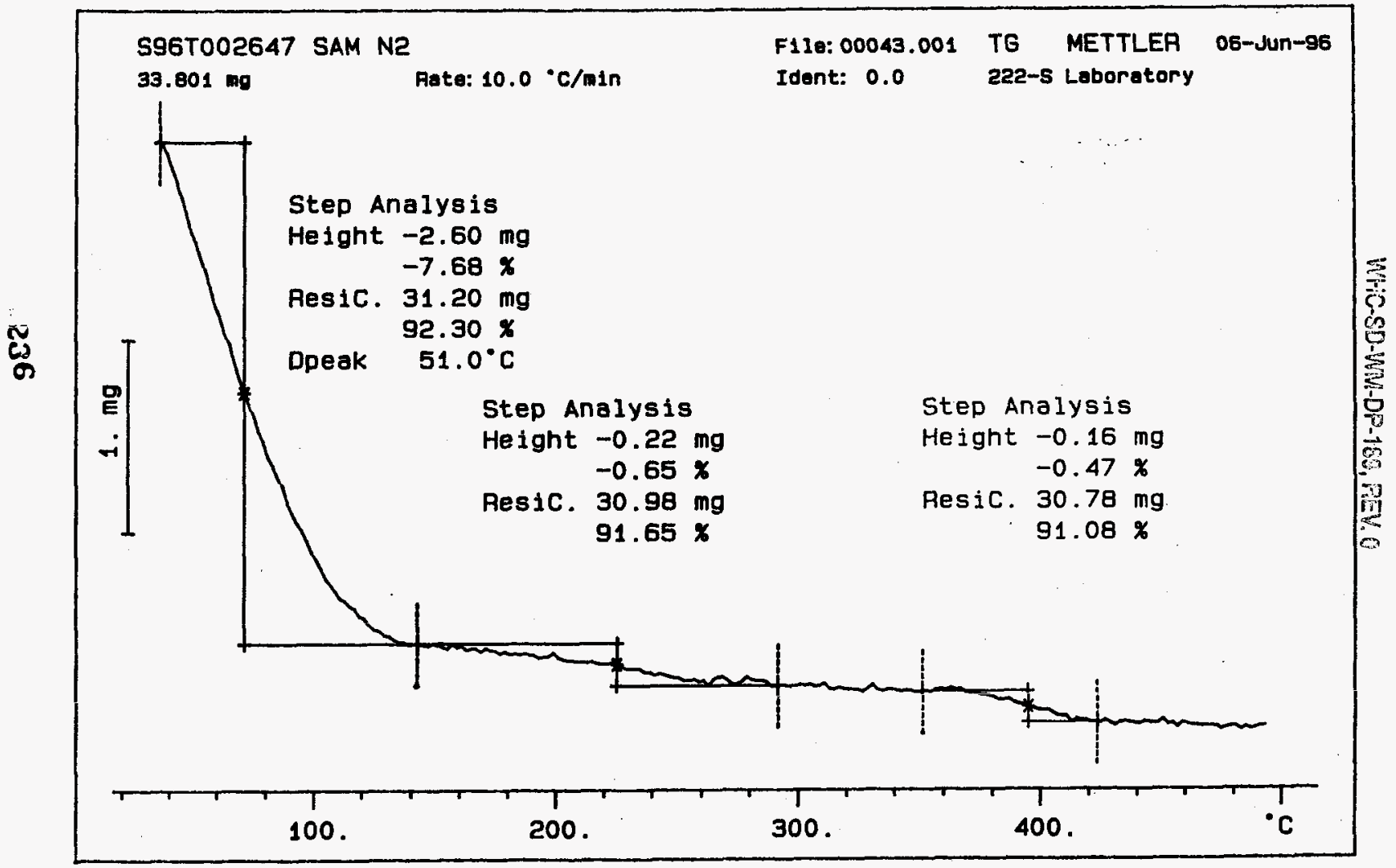




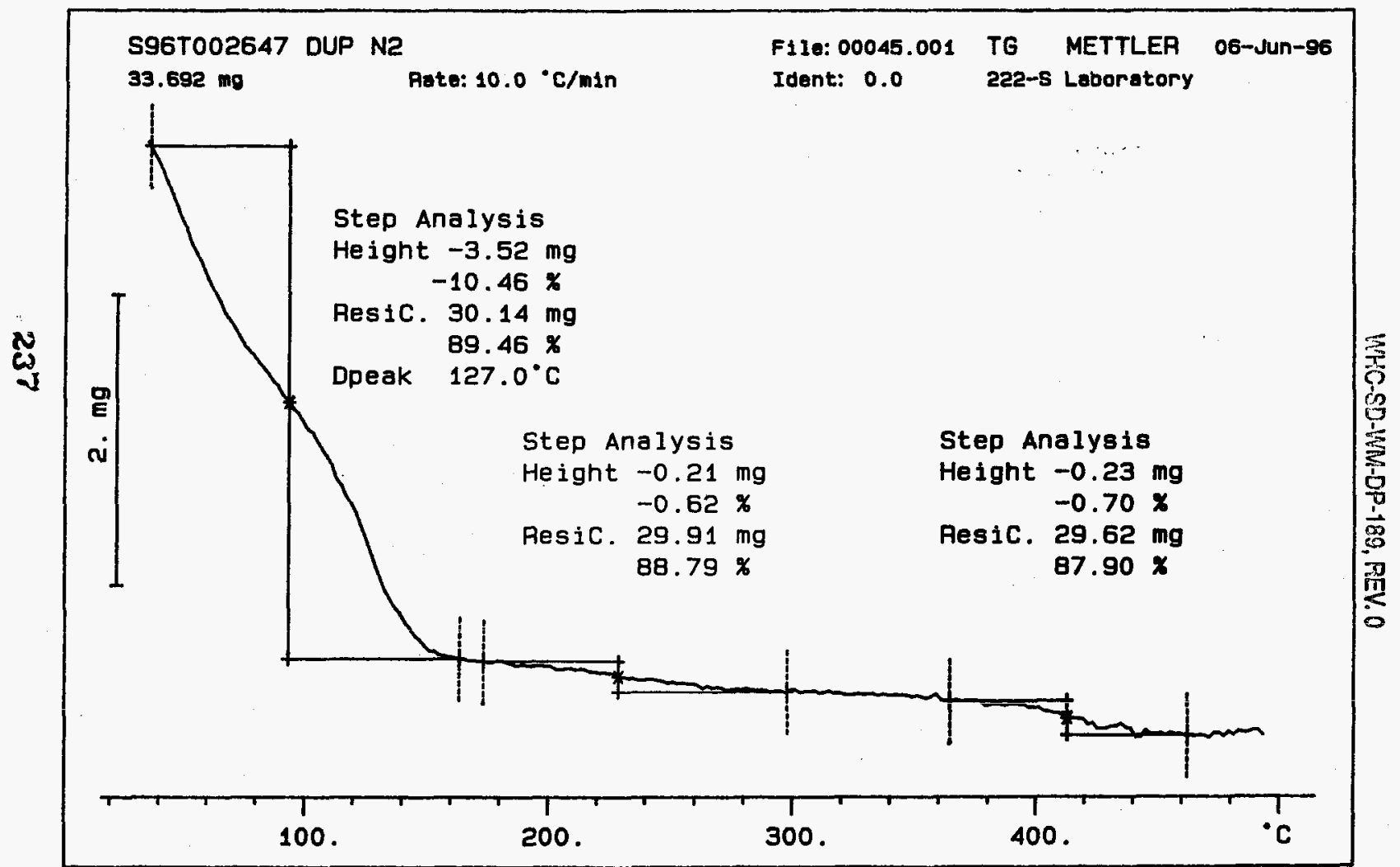




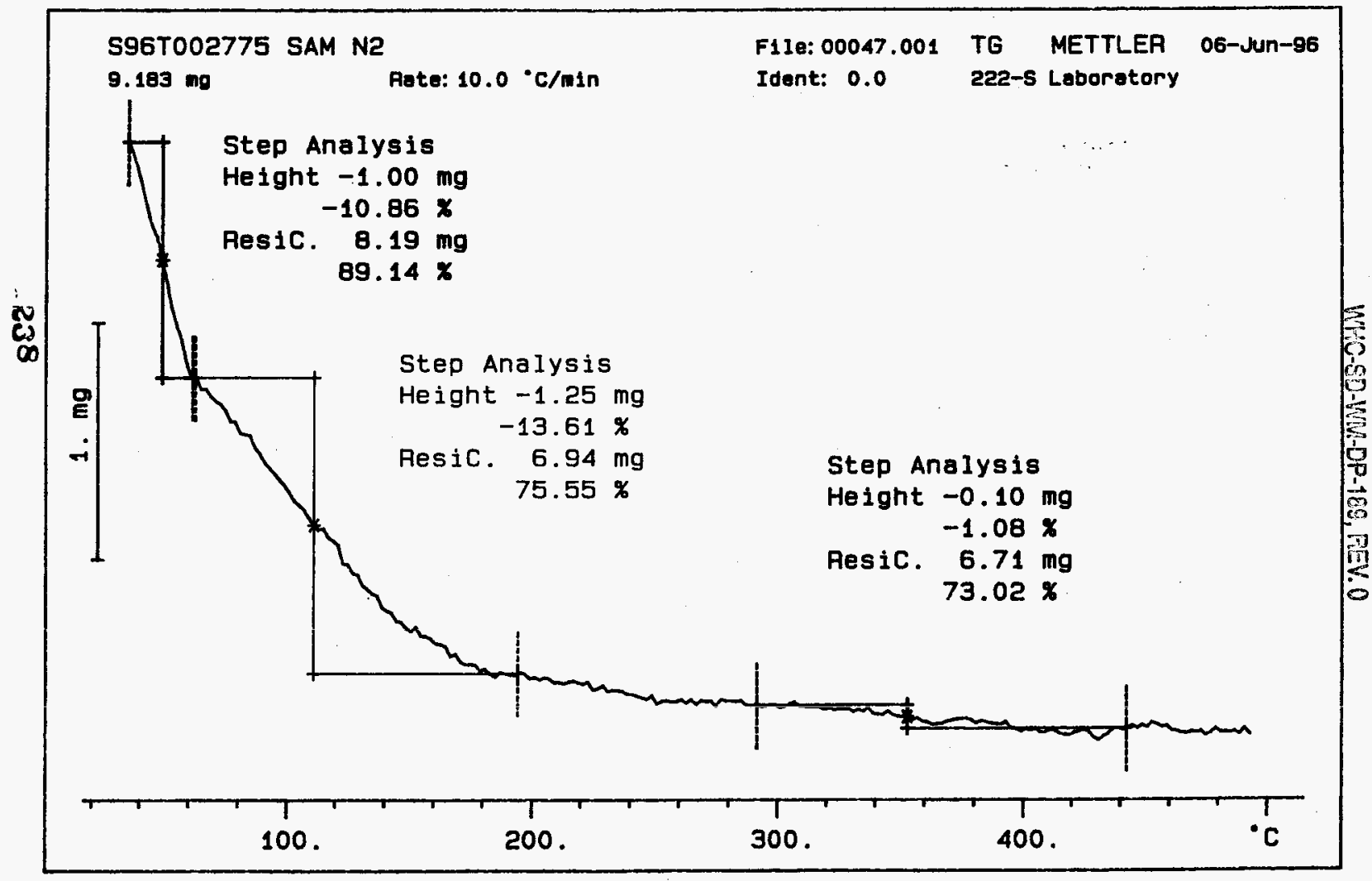




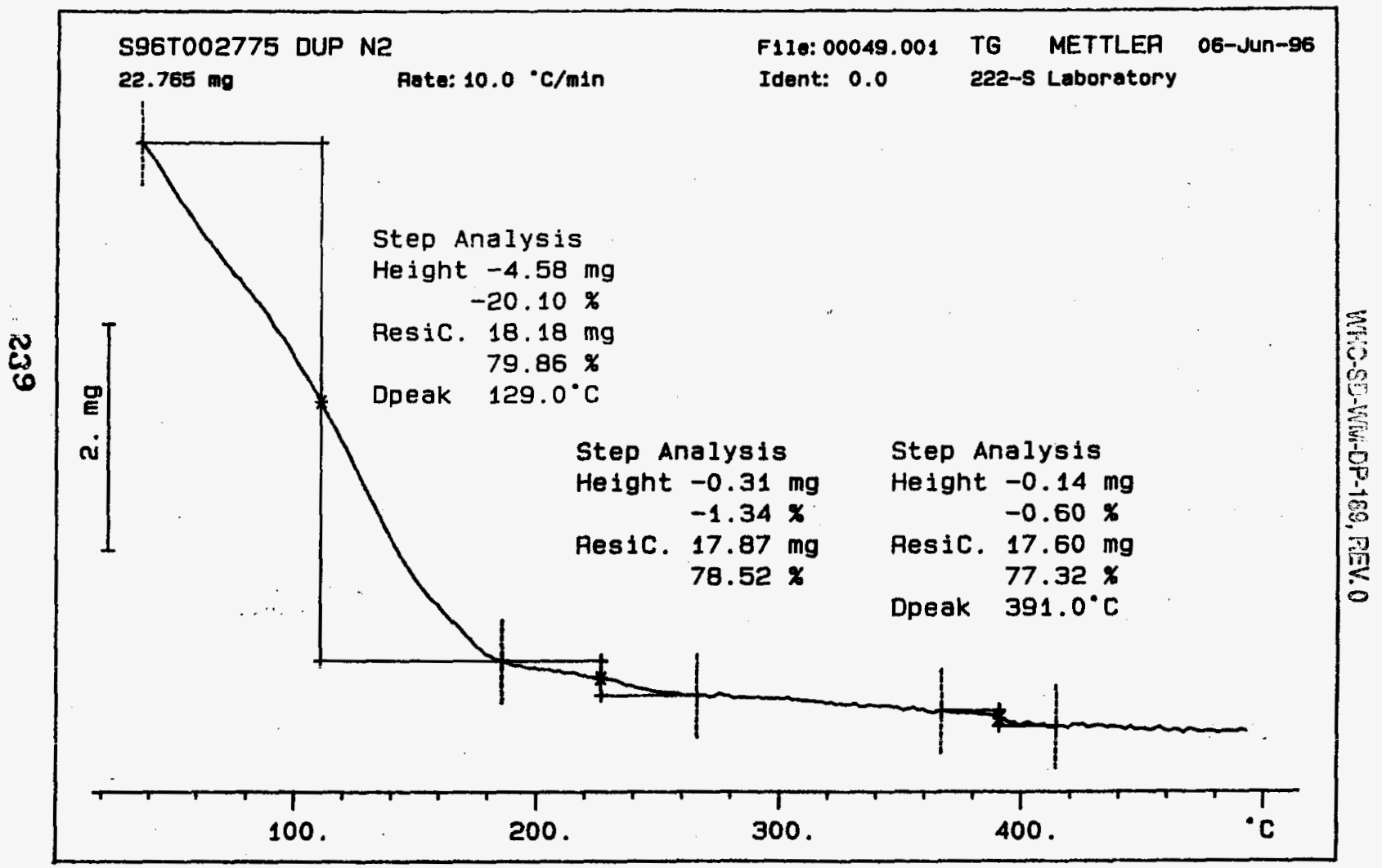


worklistrpt Version 2.1 05/15/95

WHC-SD-WM-DP-189, REV. 0

Page:

06/03/96 14:36

LABCORE Data Entry Template for Worklist\#

9534

Analyst: $D C D$ Instrument: TGA0 I Book \# 82N/BA

Method: LA-560-112 Rev/Mod B-1

Worklist Comment: U-102 TGA RUN UNDER N2. RCJ

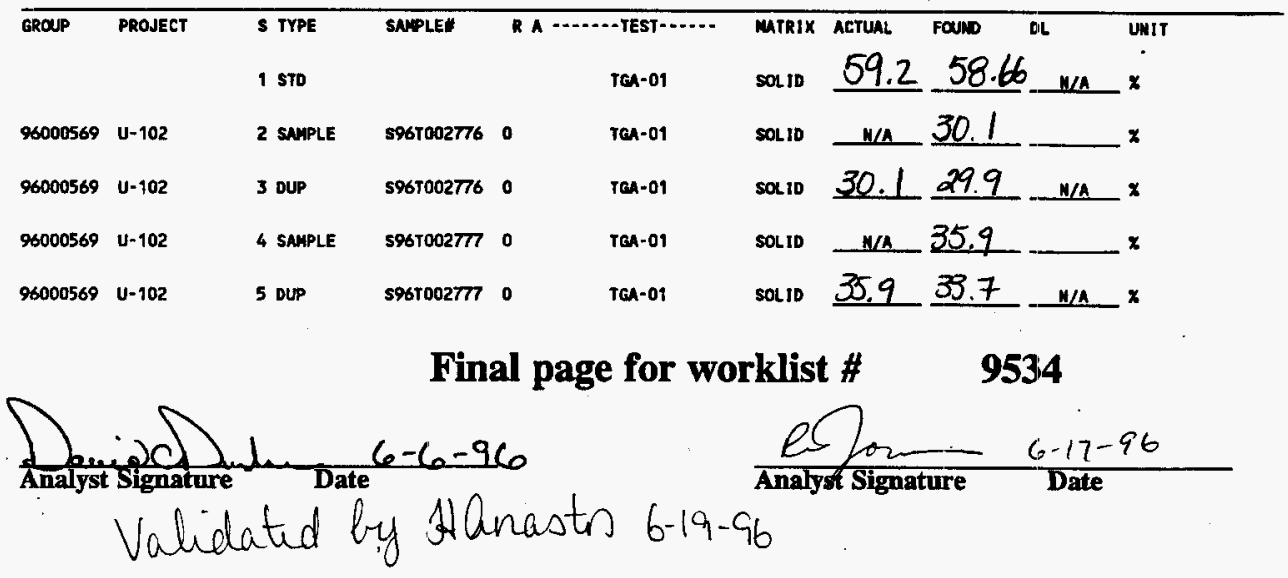

S967002776 has an ending limit of $420^{\circ} \mathrm{C}$, the data could net be hetreived from the data disk to reintegrate. The results however should not differ more than $2 \%$ if the limit were placed at approximately $200^{\circ} \mathrm{C}$.

Data Entry Comments: S967002777 has an ending limit of $420^{\circ} \mathrm{C}$. the data could not be retrained from the data disk to reintegrate. The results of the \% moisture should not be different of more than $2 \%$

Units shown for $Q C$ (SPR \& STD) may not reflect the actual wits. $D L=$ Detection Limit, $S=$ Wortlist Slot Number, $R=$ Replicate Number, $A=$ Aliquot Code.

240 
SIGNATURE BELOW REPRESENTS CHEMICAL TECHNOLOGIST/CHEMIST THAT

COKPLETED/VERIFIED THE CALIBRATION/ANALYSIS ON PAGES 241 TO 245.

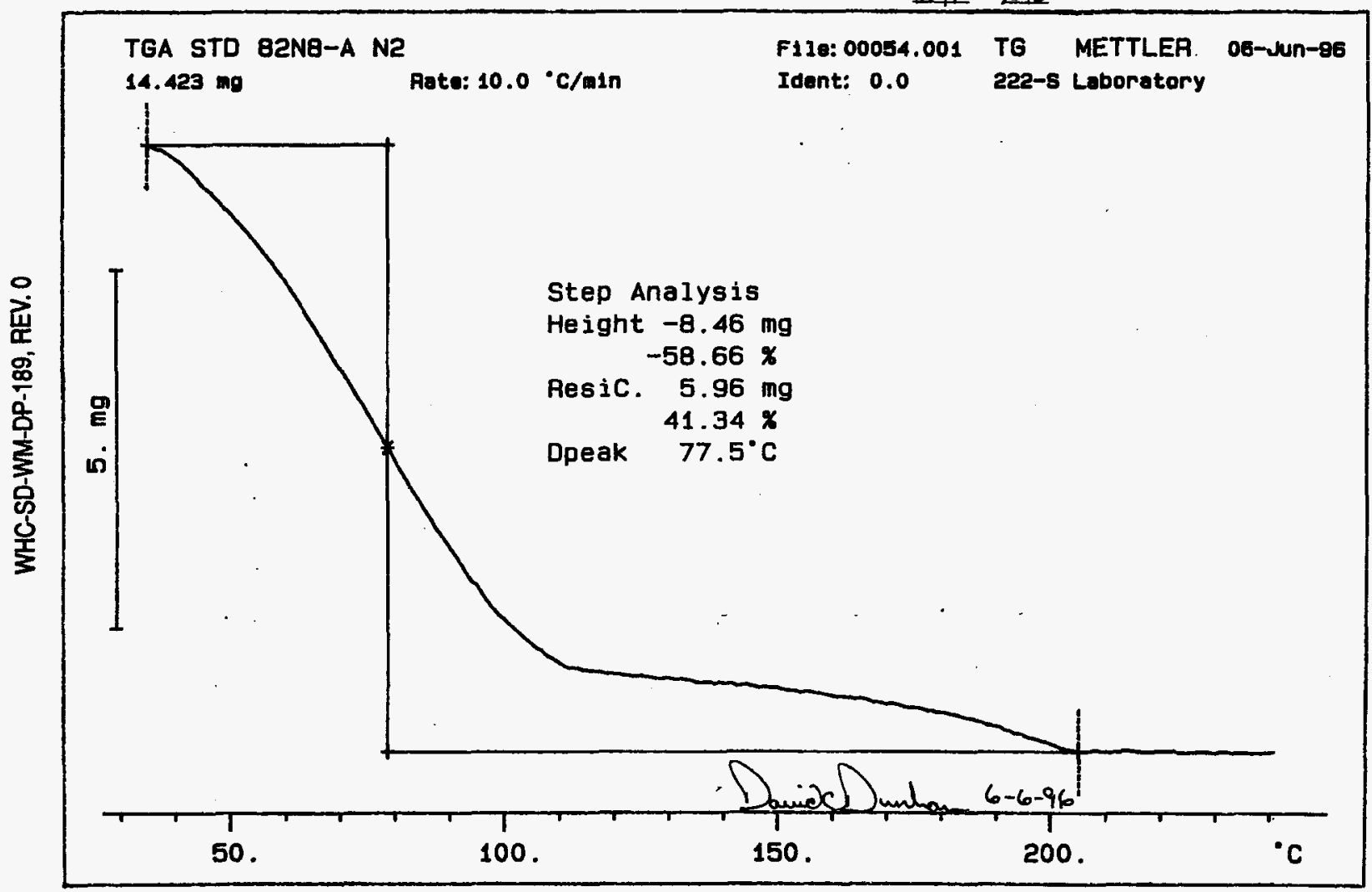




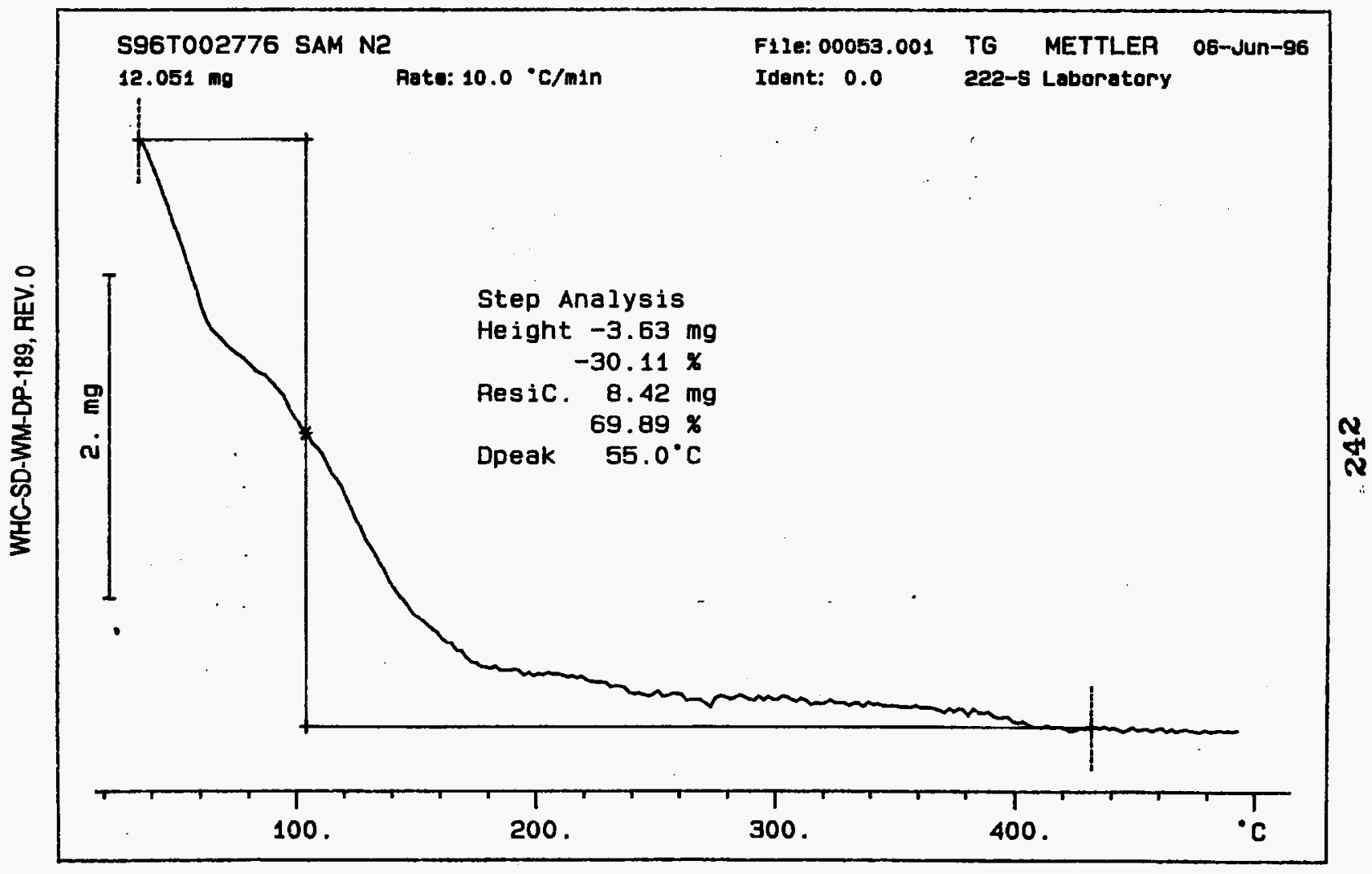




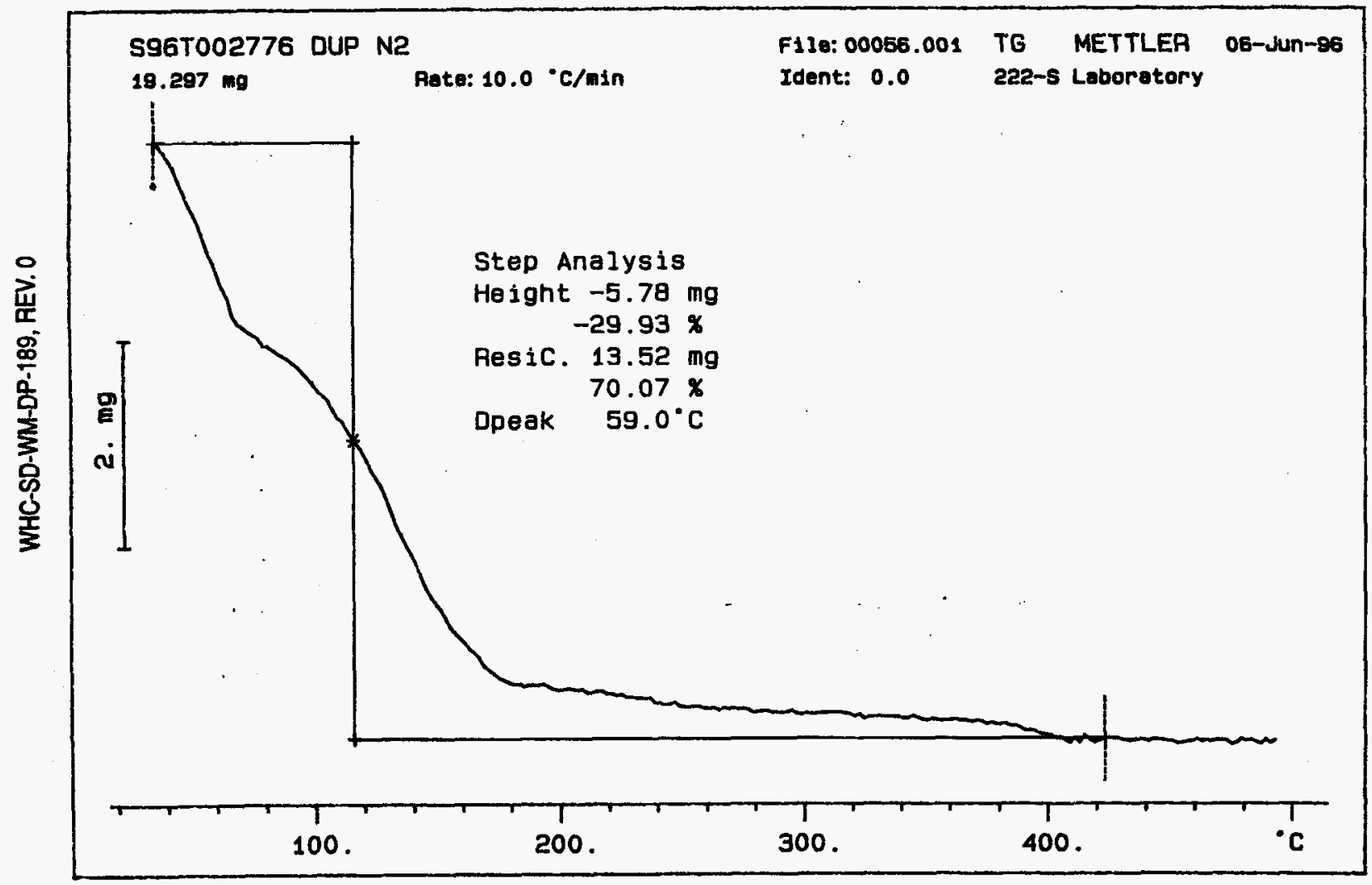




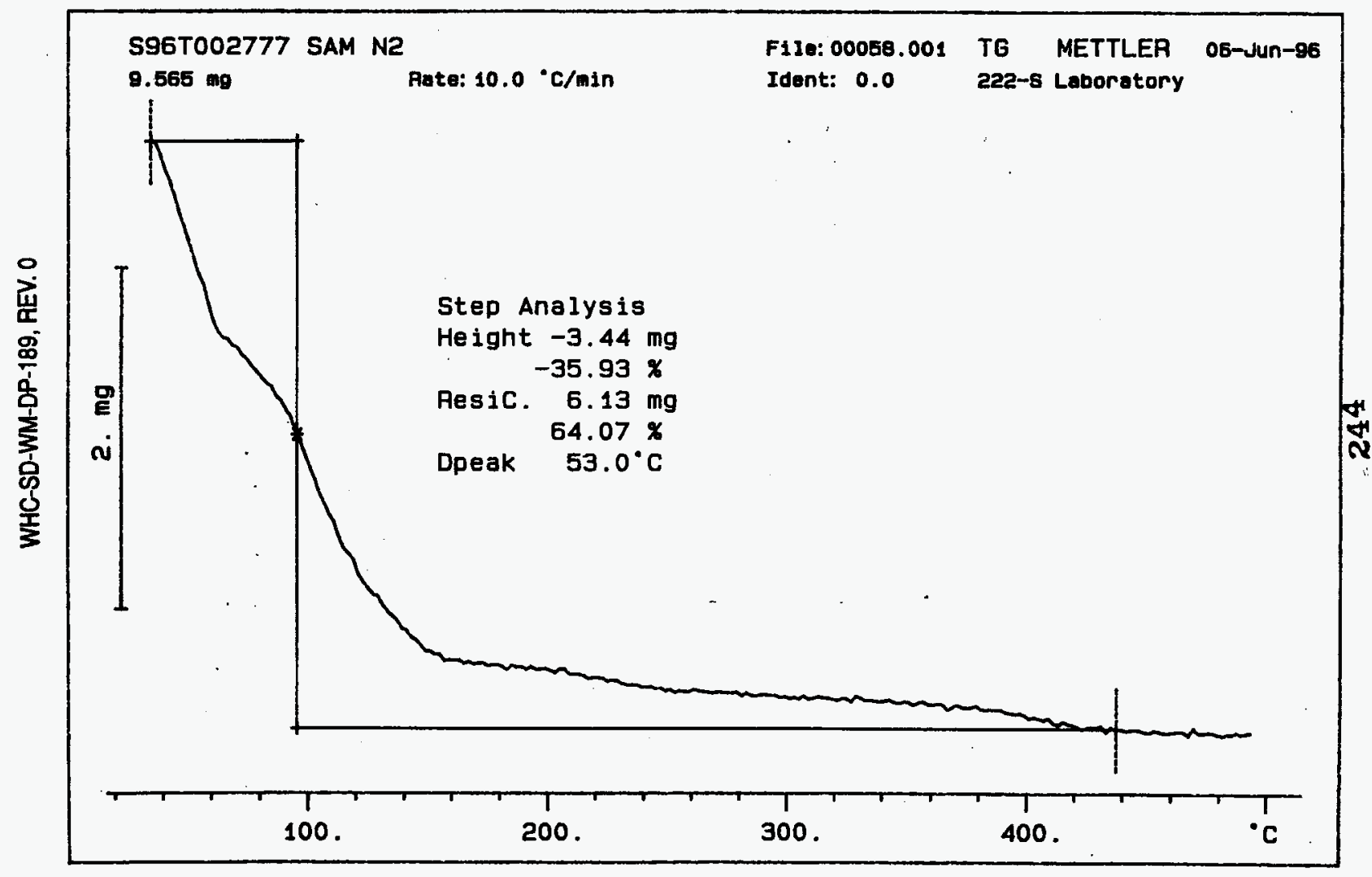




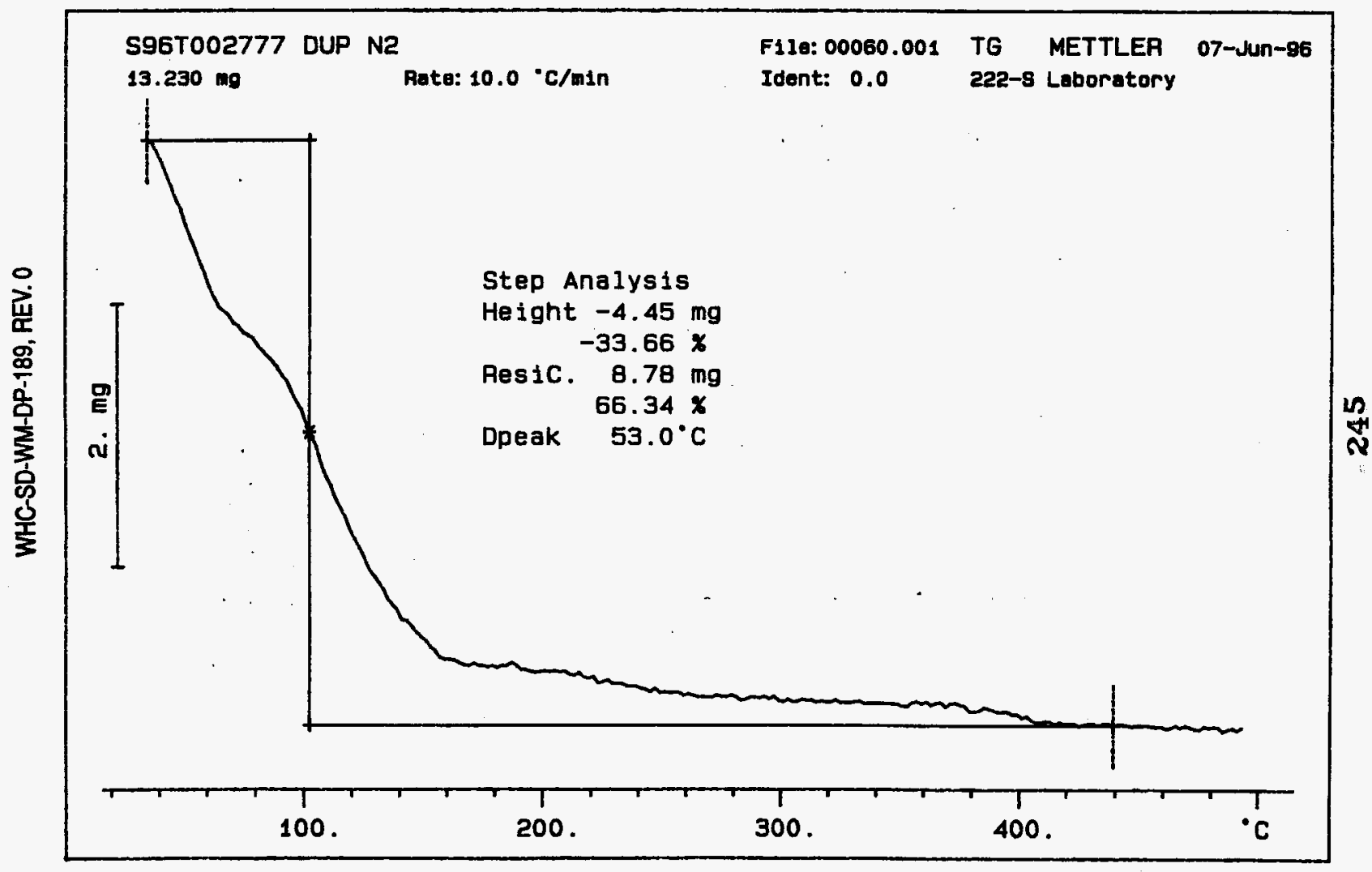




\section{LABCORE Data Entry Template for Worklist\#}

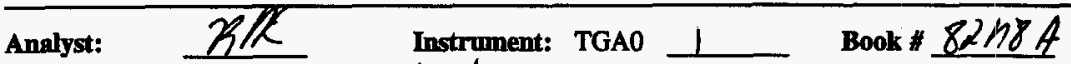

Method: LA-560-112 Rev/Mod Bu

Worklist Comment: U-102 TGA RUN UNDER N2. RCJ

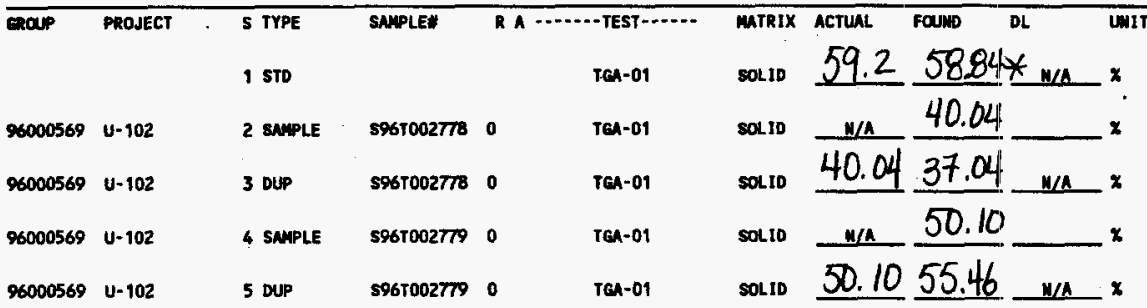

\section{Final page for worklist \#}

9535

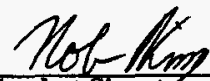

Analyst Signature

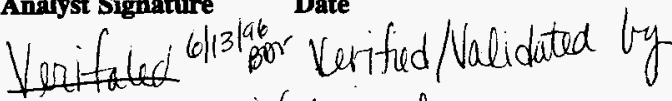

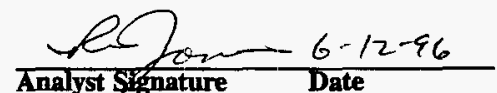

Datr Bury commats: S967002778 results are the sum of two weight loss steft

Units shown for $Q C$ (SPK \& STD) may not reflect the actual units. $D L=$ Detection Limit, $S=$ Worklist Slot Number, $R=$ Replicate Number, $A=$ Aliquot Code. 
SIGNATURE BELOW REPRESENTS CHEMICAL. TECHNOLOGI'ST/CHEMIST THAT

COMPLETED/VERIFIED THE CALIBRATION/ANALYSIS ON PAGES 247 TO 251.

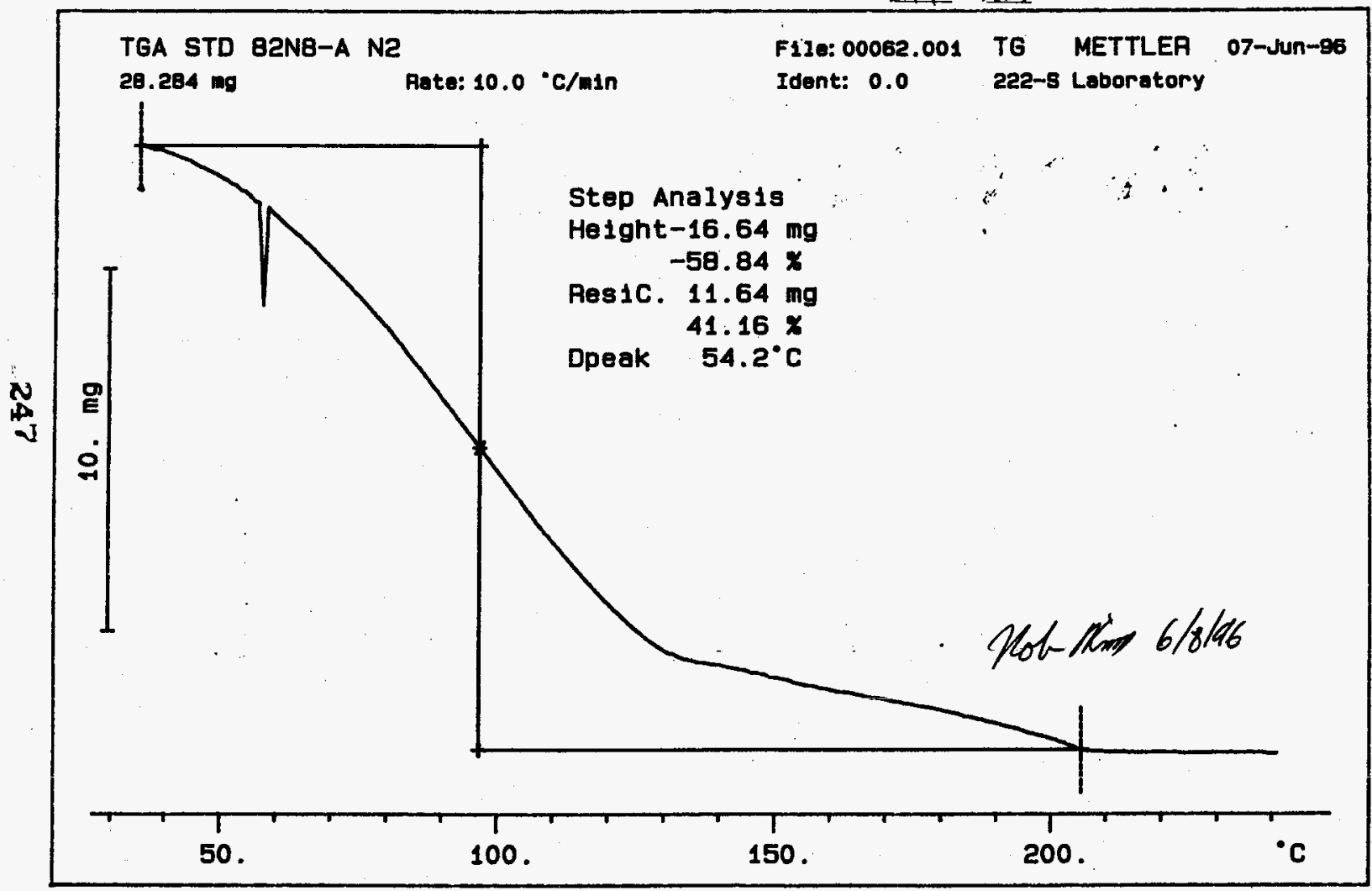

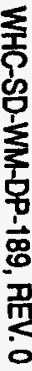




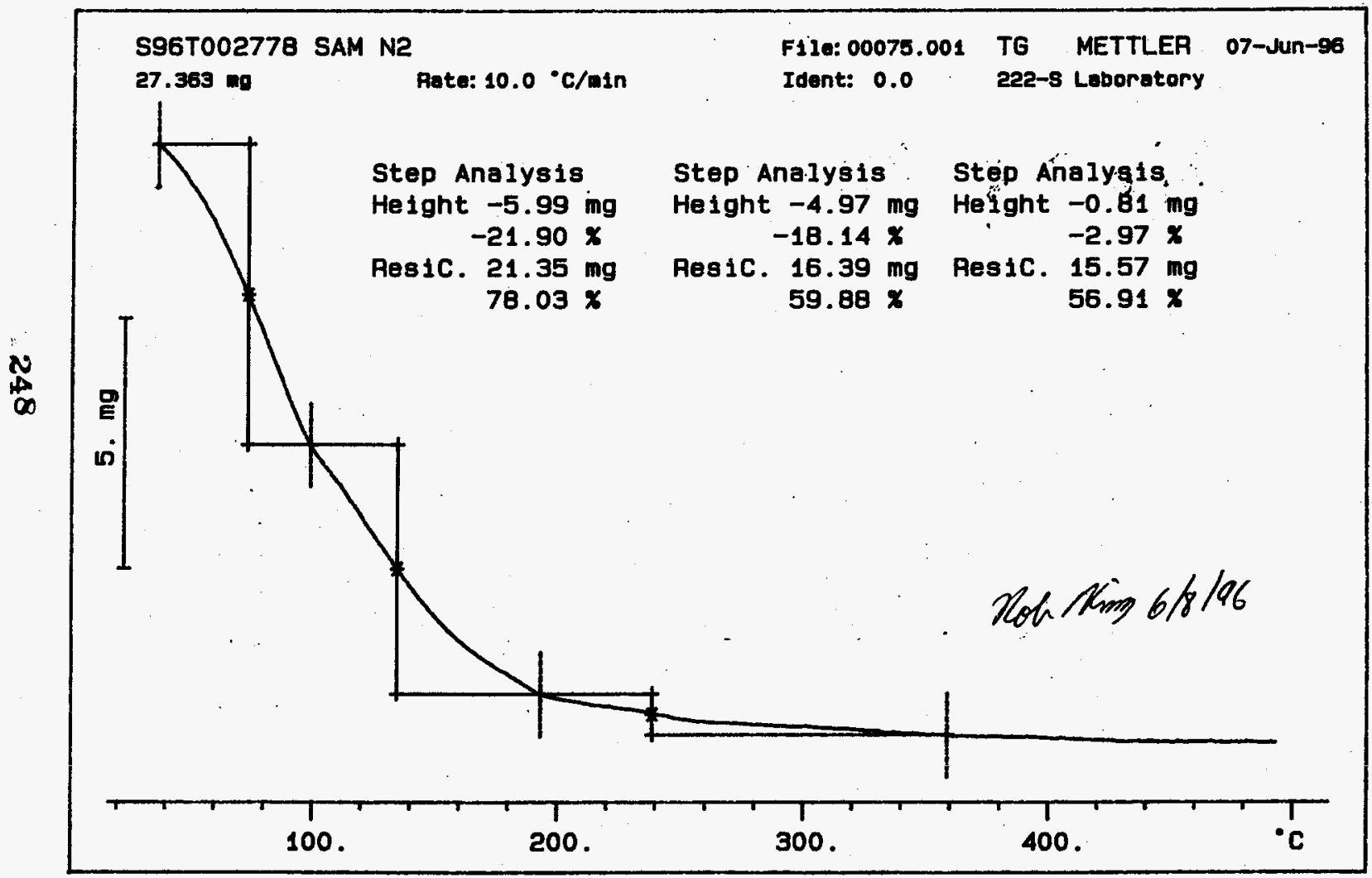




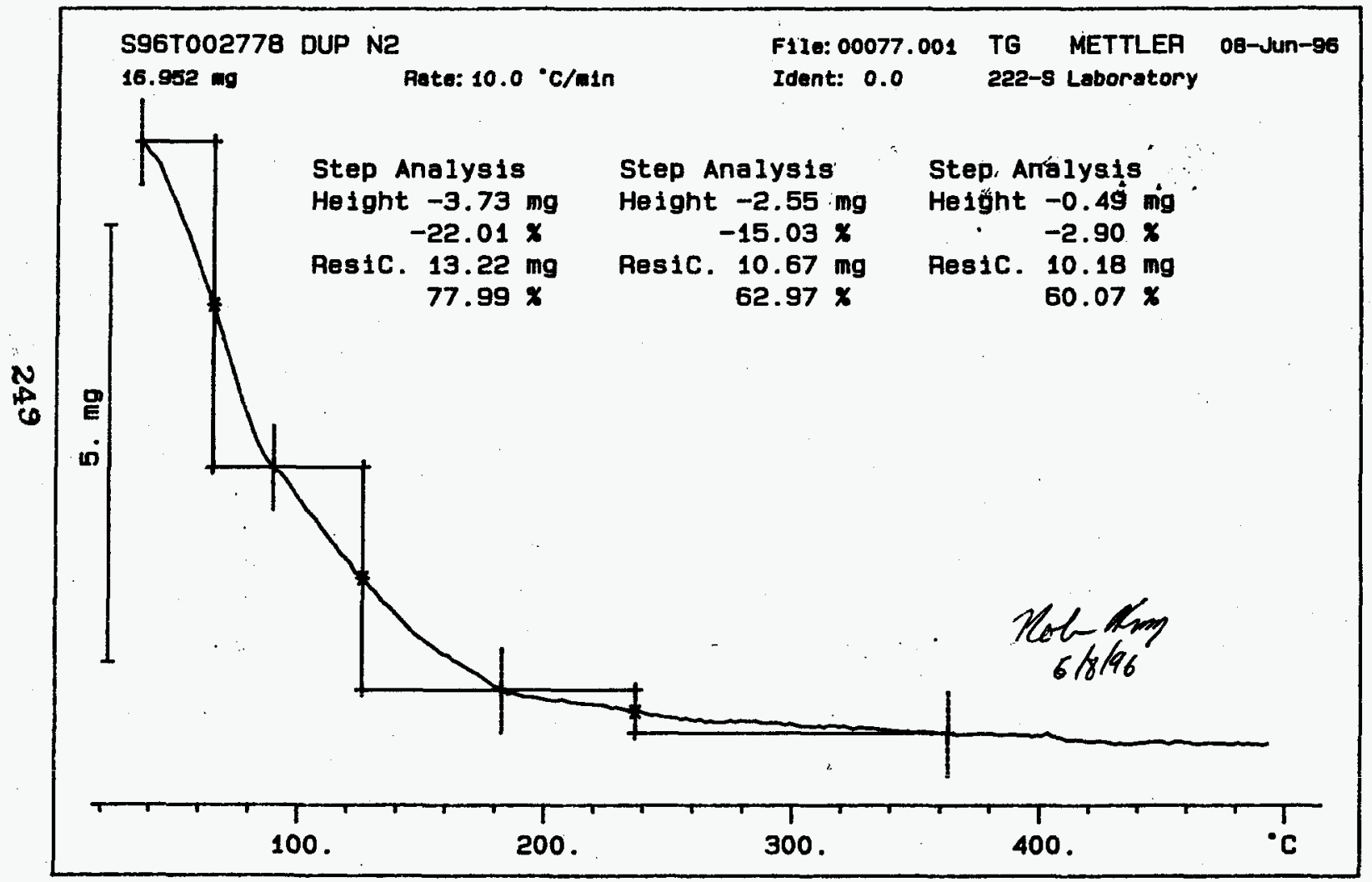




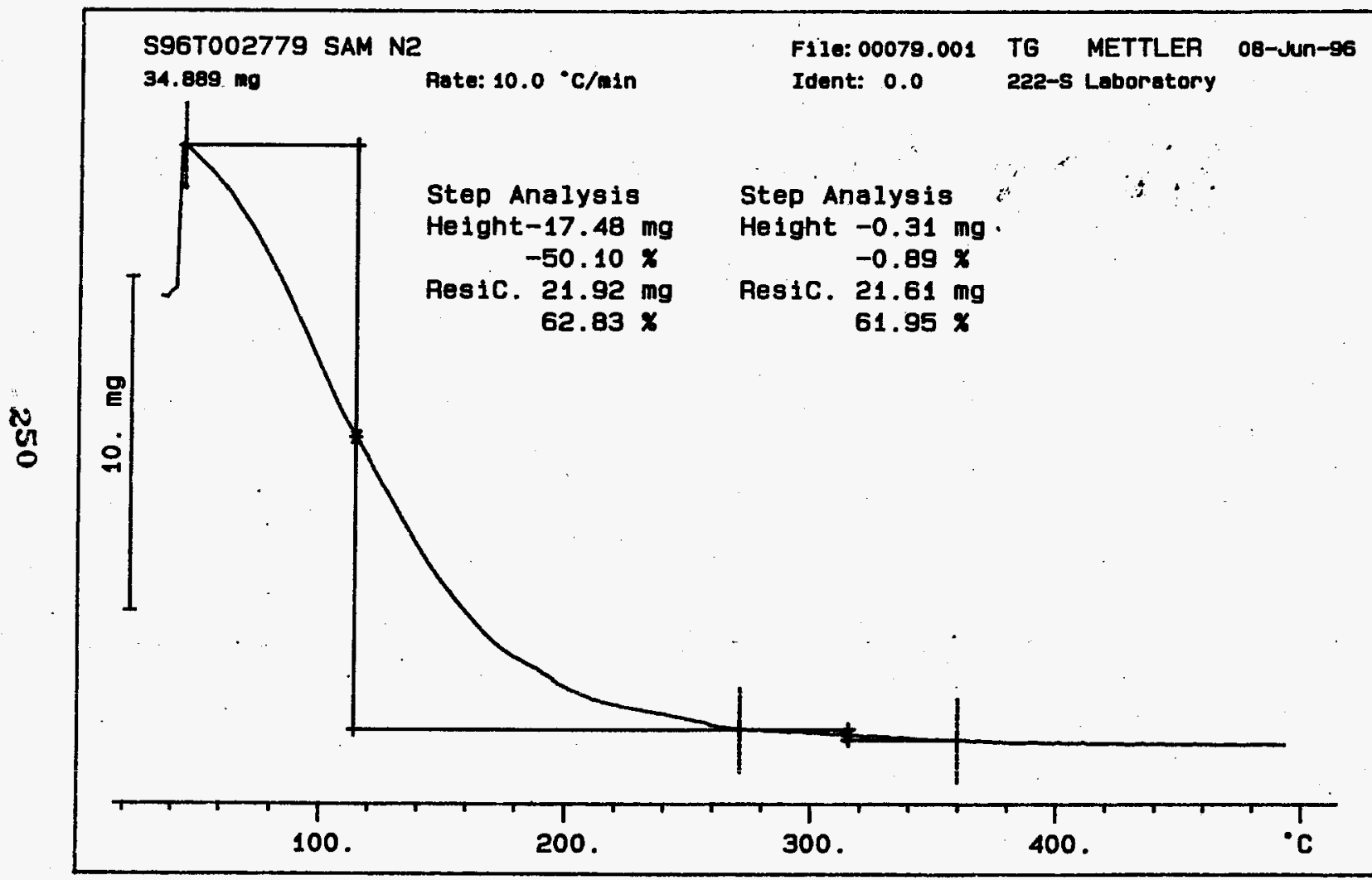




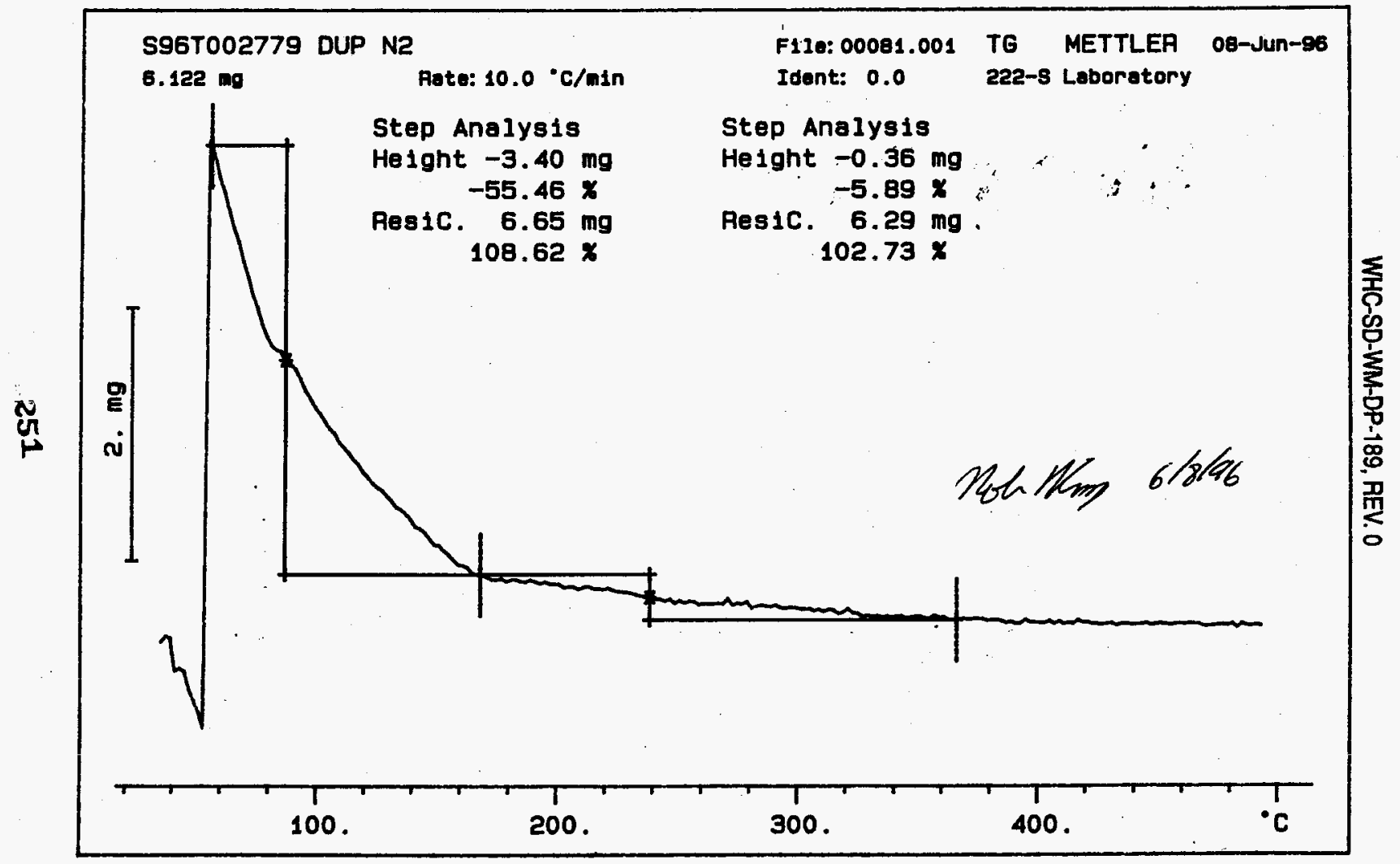


worklistrpt Version 2.1 05/15/95

WHC-SD-WM-DP-189, REV. 0

Page: 1 $06.03 / 9614: 42$

LABCORE Data Entry Template for Worklist\#

9536

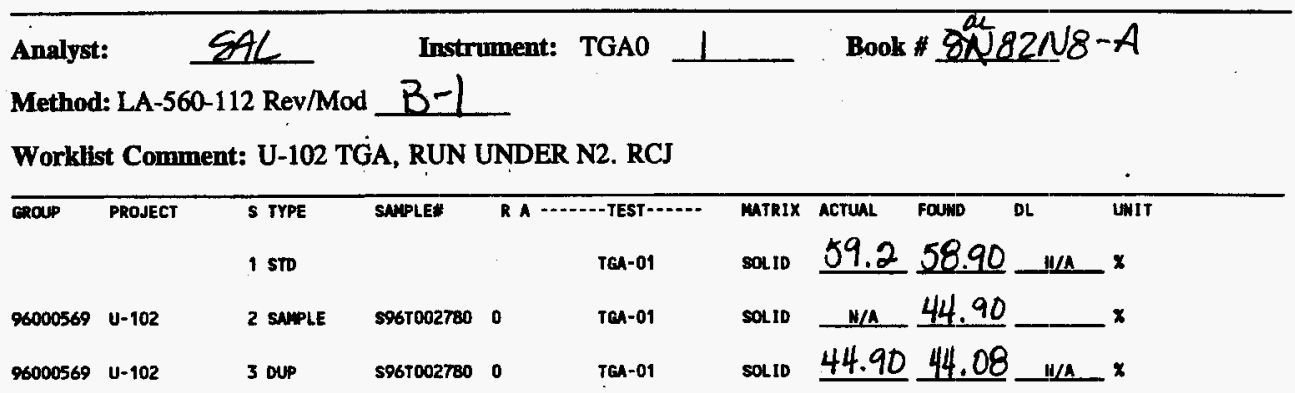

Final page for worklist \# 9536

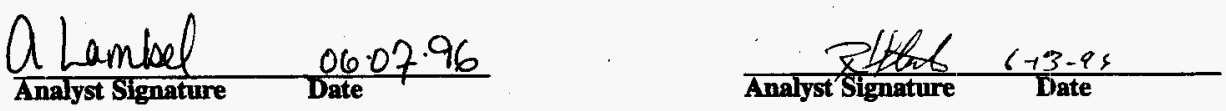

Verified/Validated by

Blandina

Valenzuela $6 \cdot 14-96$

Data Entry Comments:

Units shown for $Q C$ (SPK \& STD) may not reflect the actual units. $D L=$ Detection Limit, $S=$ Worklist Slot Number, $R=$ Replicate Number, $A=$ Aliquot Code.

252 


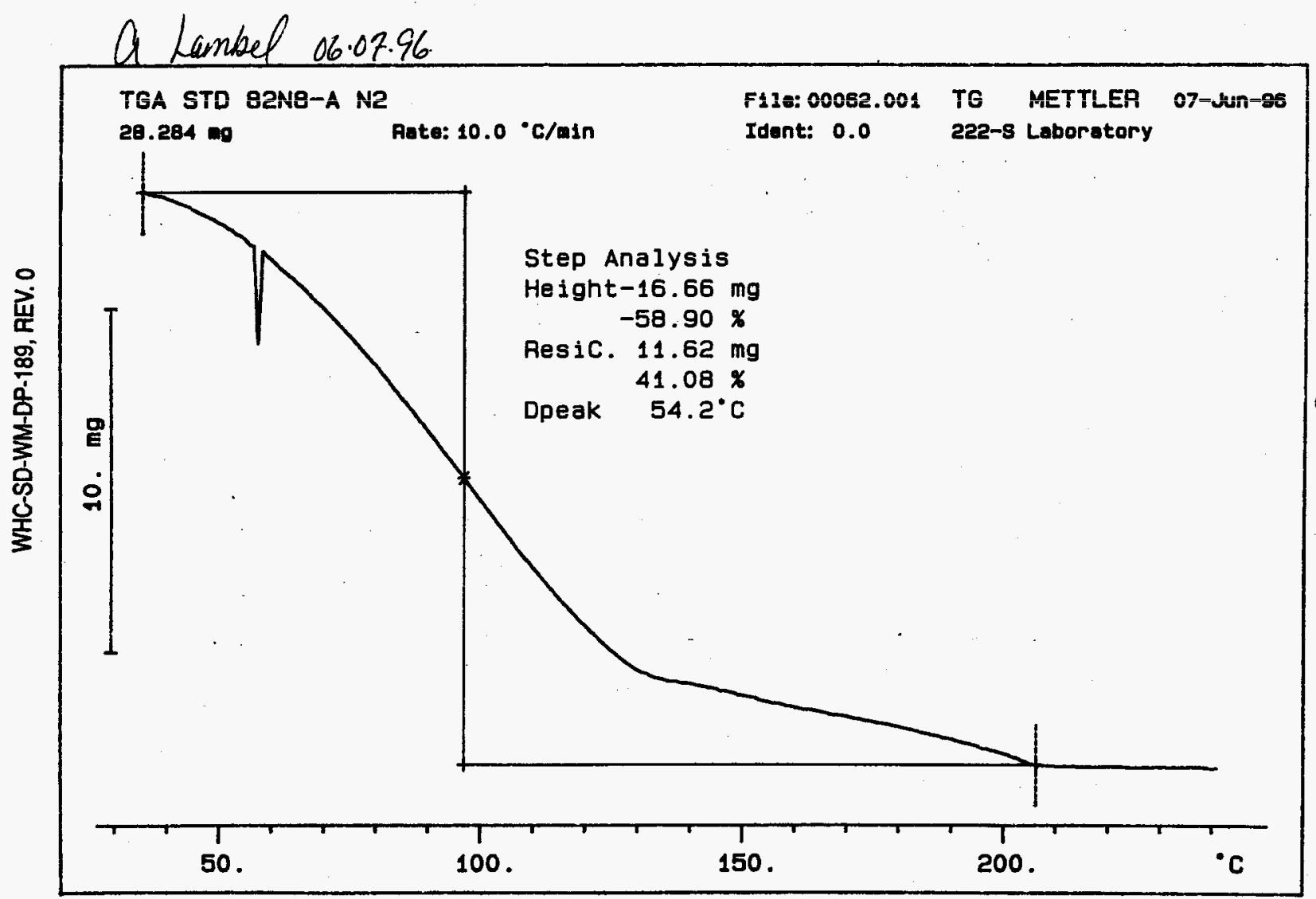

ก

SIGNATURE ABOVE REPRESENTS CHEMICAL TECHNOLOGIST/CHEMIST THAT COMPLETED/VERIFIED THE CALIBRATION/ANALYSIS ON PAGES 253 TO 255 


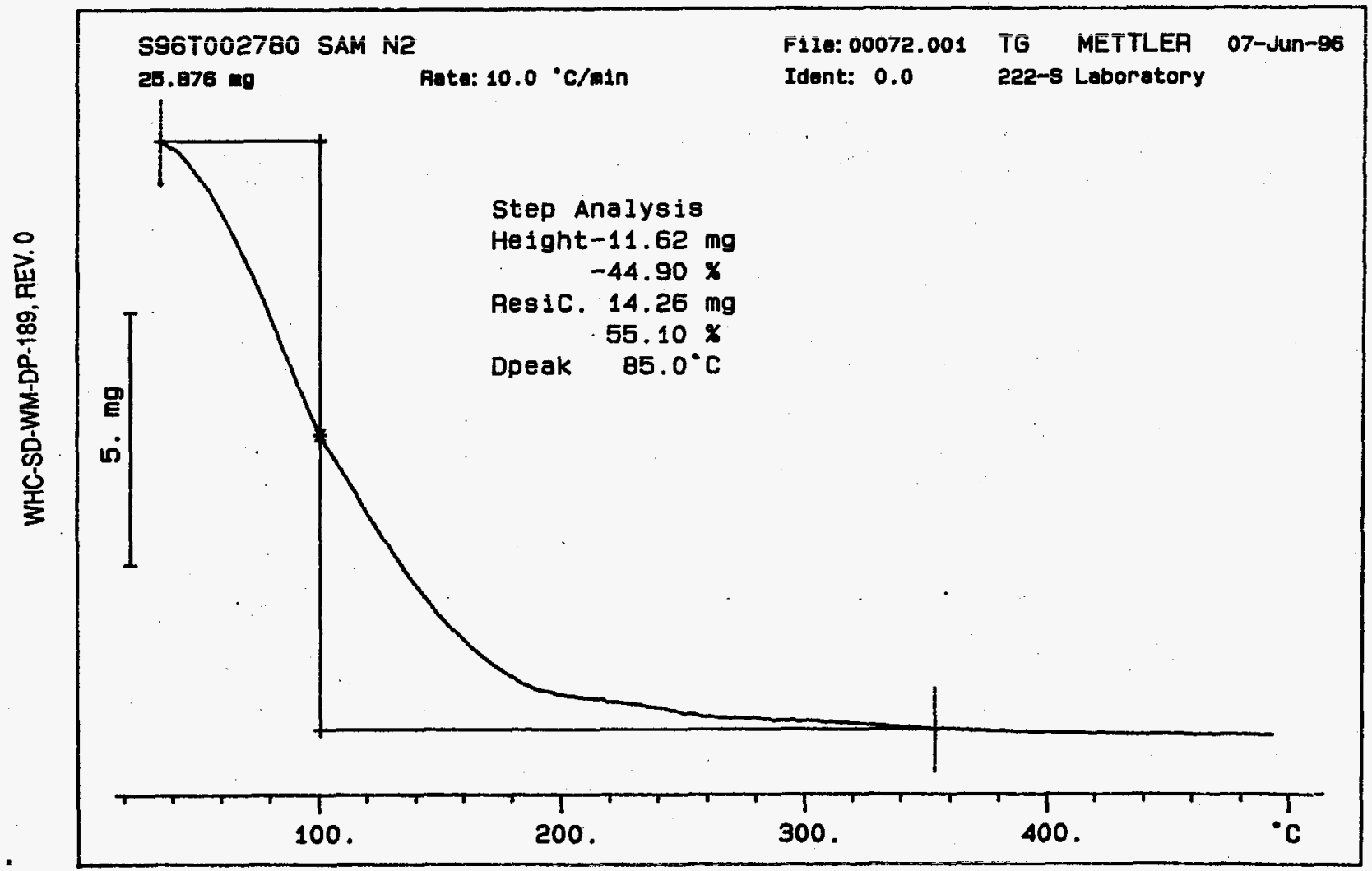




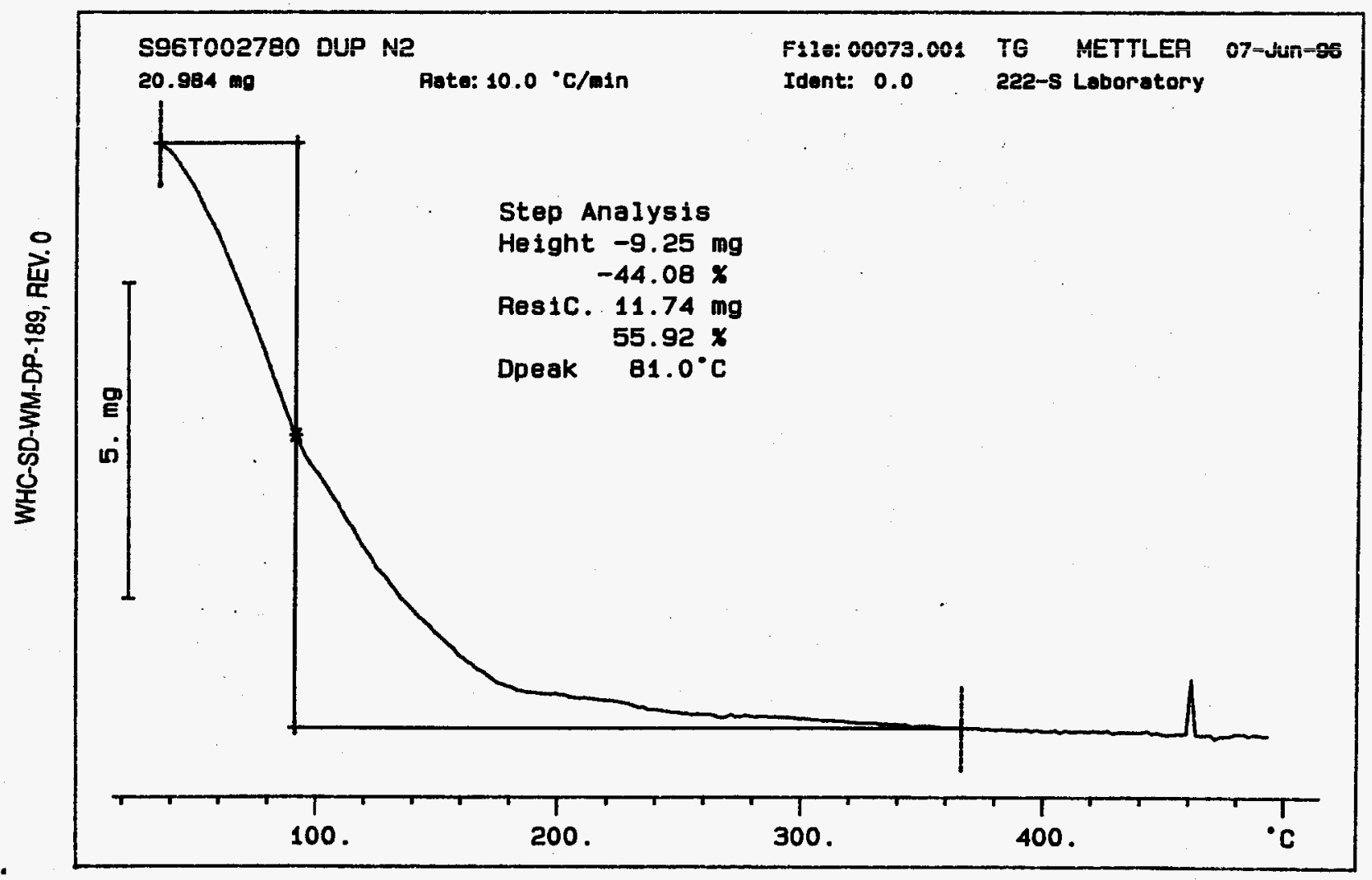


WHC-SD-WM-DP-189, REV. 0

Page: I

worklistrpt Version $2.105 / 15 / 95$

LABCORE Data Entry Template for Worklist;

10074

Analyst: HAP Instrument: TGA0 $\perp$ Book \# 8 N $8 A$

Method: LA-514-114 Rev/Mod B-1

Worklist Comment: TGA U-102 Reruns. Run under N2. RUSH

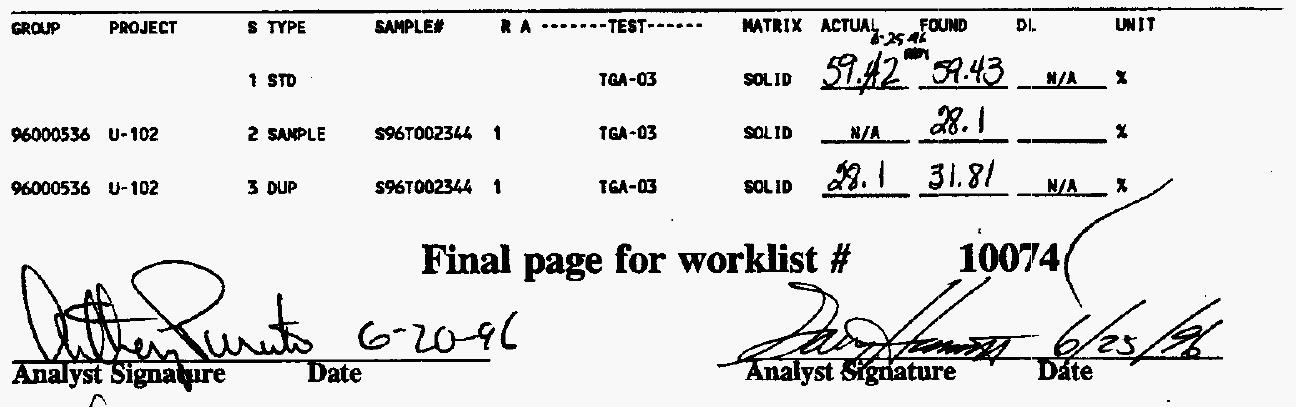

Verified/ Validate by

Blundina

Valenzuela

$6-26-96$

Date Eur cones: Sample results are the sum of two wright loss steps.

Units shown for QC (SPK \& STD) may not reflect the actual units. $D L=$ Detection Limit, $S=$ Worklist Slot Number, $R=$ Replicate Number, $A=$ Aliquot Code.

256 
SIGNATURE BELOH REPRESENTS CHEMICAL TECHNOLOGIST/CHEMIST THAT

COMPLETED/VERIFIED THE CALIBRATION/ANALYSIS ON PAGES 257 TO 259

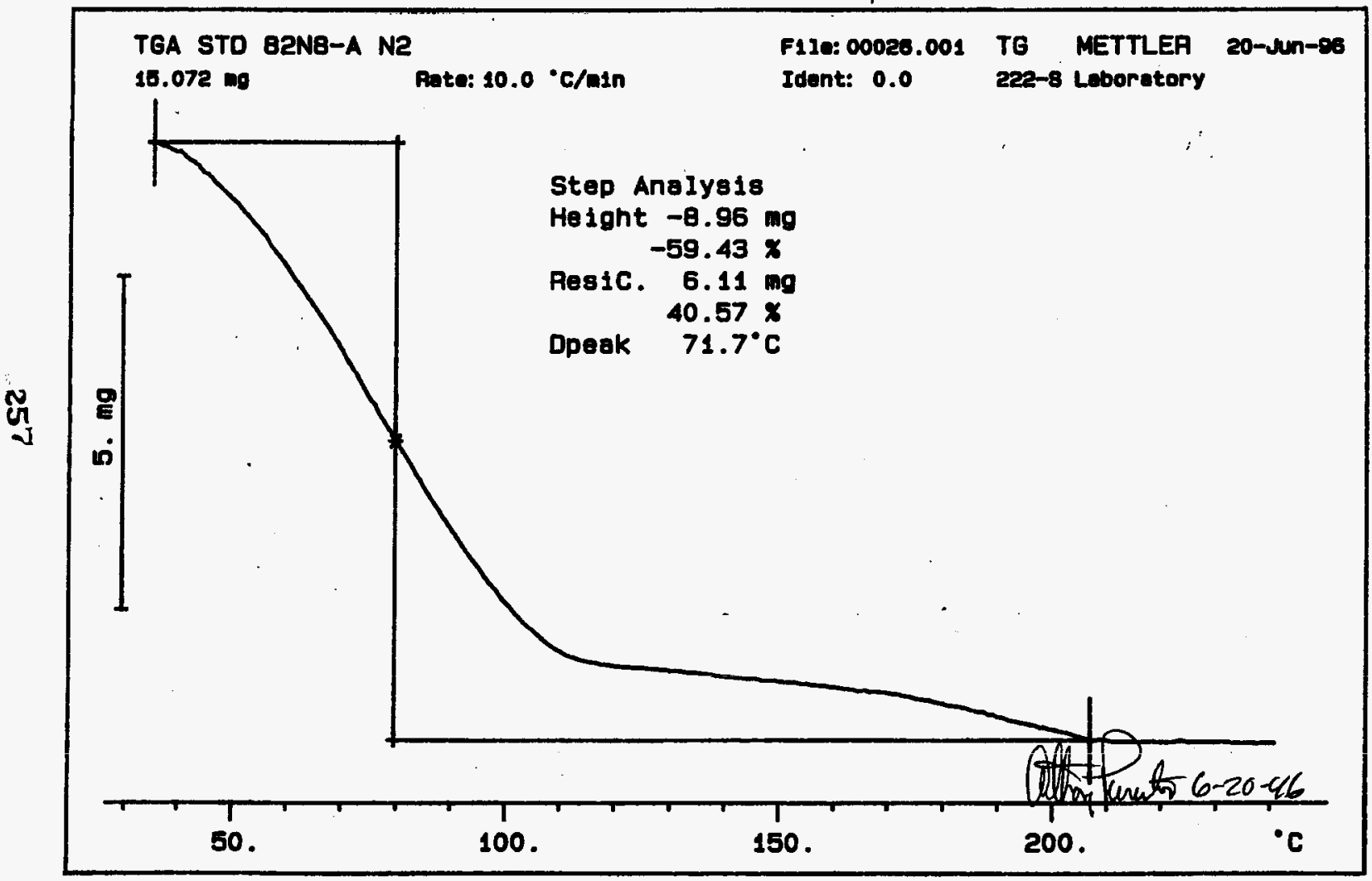




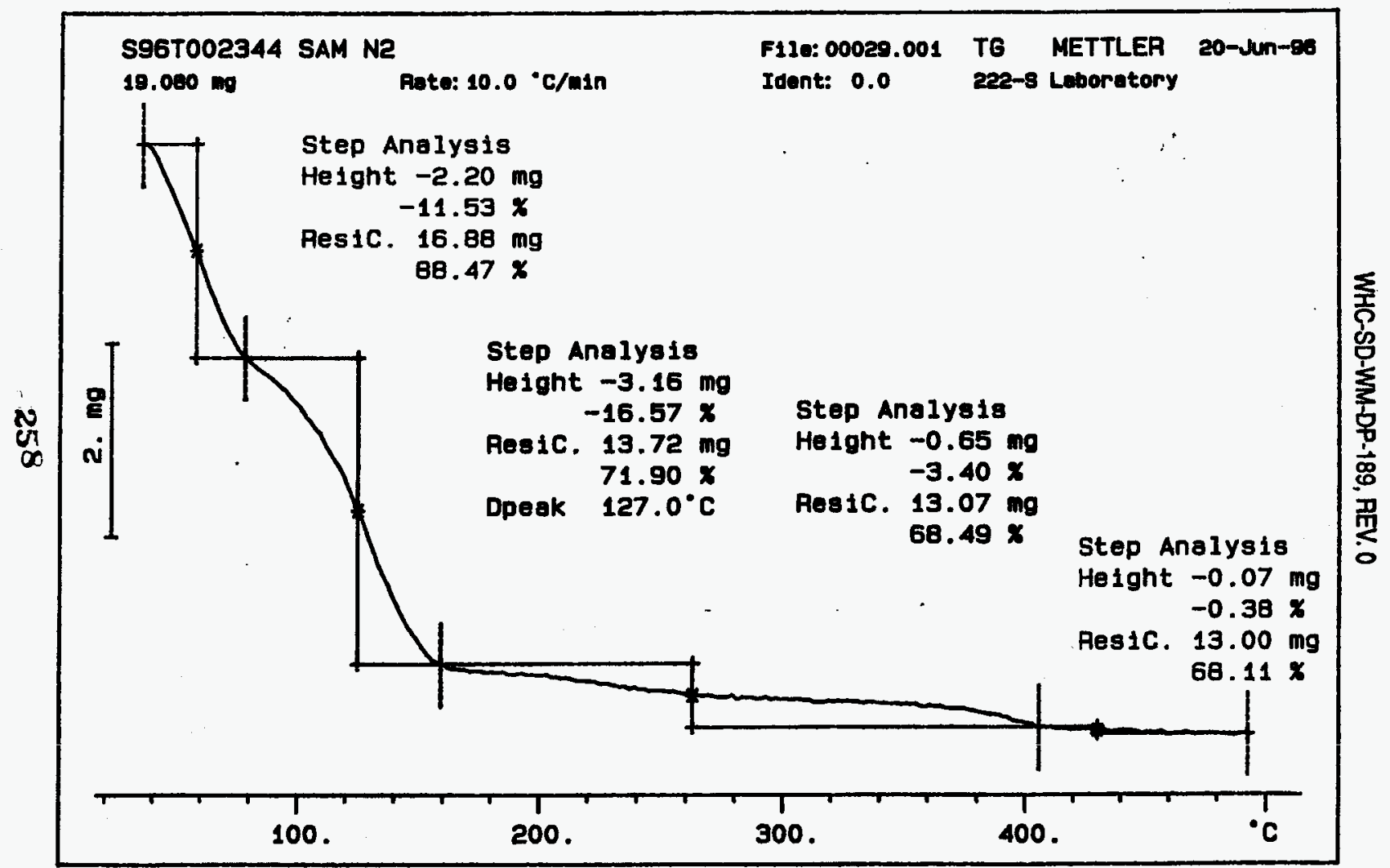




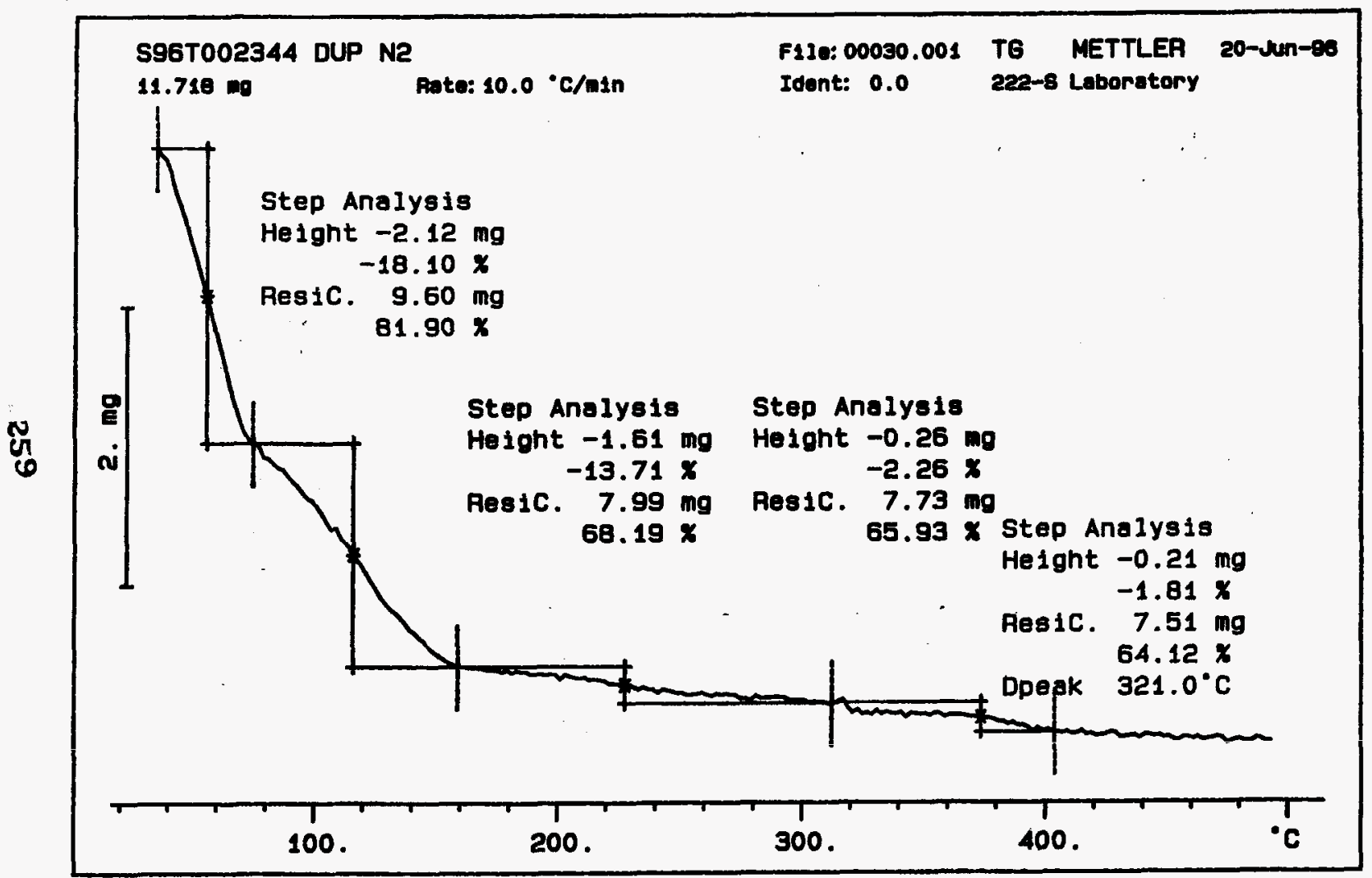




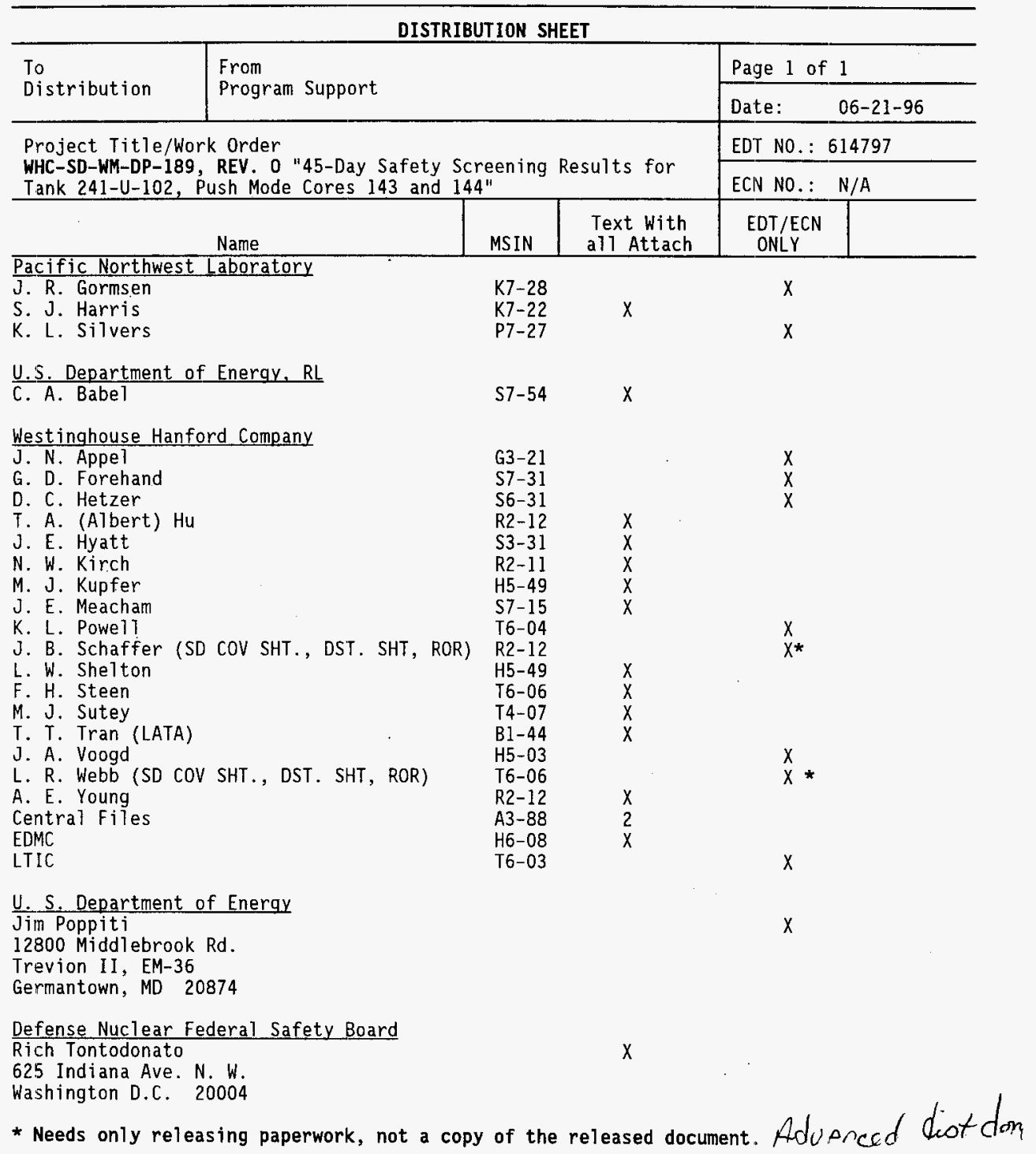

